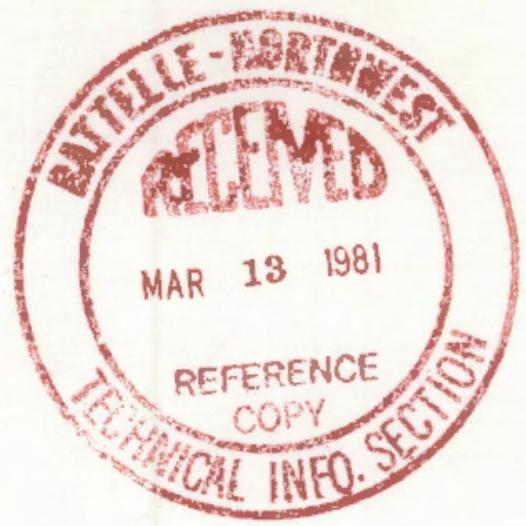

PNL-3711

1-

ISPO-139

PNL-3711

UC-15

ISPO TASK C.19

\title{
ANALYSIS OF THE IMPACT OF SAFEGUARDS CRITERIA
}

JANUARY 1981

\section{$\frac{\text { USA }}{\text { IAEA }}$}

\section{PROGRAM FOR TECHNICAL ASSISTANCE TO IAEA SAFEGUARDS}

Department of Energy

Office of Safeguards \& Security

M. F. Mullen

P. T. Reardon

PACIFIC NORTHWEST LABORATORY OPERATED BY BATTELLE MEMORIAL INSTITUTE 


\title{
NOTICE
}

This report was prepared as an account of work sponsored by an Agency of the United States Government. Neither the United States Government nor any Agency thereof, nor any of their employees, nor any of the contributors to this document, makes any warranty, expressed or implied, or assumes any legal liability or responsibility for any third party's use, or the results of such use, of any information, apparatus, product or process disclosed in this report, or represents that its use by such third party wouid not iniringe privately owned rights. Further, neither the su'bject matter nor the content of this report reflects any policy, expressed or implied, of the United States Government.

\author{
PACIFIC NORTHWEST LABORATORY \\ operated by \\ BATTELLE \\ for the \\ UNITED STATES DEPARTMENT OF ENERGY \\ Under Contract DE-AC06-76RLO 1830
}
Printed in the United States of America Available from
National Technical Information Service
United States Department of Commerce
5285 Port Royal Road
Springfield, Virginia 22151

Price: Printed Copy $\$$

*; Microfiche $\$ 3.00$

NTIS

*Pages Selling Price

$001-025 \quad \$ 4.00$

026-050 \$4.50

051-075 $\$ 5.25$

076-100 $\$ 6.00$

$101-125 \quad \$ 6.50$

$126-150 \quad \$ 7.25$

$151-175 \quad \$ 8.00$

$176-200 \quad \$ 9.00$

201-225 $\$ 9.25$

226-250 $\$ 9.50$

251-275 \$10.75

276-300 $\$ 11.00$ 
ISPO- 139

PNL -3771

UC -15

ANALYSIS OF THE IMPACT

OF SAFEGUAROS CRITERIA

M.F. Mullen

P.T. Reardon

January 1981

Prepared for the International Safeguards Project Office Program for Technical Assistance to the International Atomic Energy Agency

Pacific Northwest Laboratory

Richland, Washington 99352 
SUMAARY

As part of the U.S. Program of Technical Assistance to IAEA Safeguards, the Pacific Northwest Laboratory (PNL) was asked to assist in developing and demonstrating a model for assessing the impact of setting criteria for the appiication of IAEA safeguards. This report presents the resuits of PNL'S work on the task.

The report is in three parts. The first explains the technical approach and methodology. The second contains an example application of the methodology. The third presents the conclusions of the study.

The technical approach involves the following activities. The first requirement is to define a model that relates the impacts and the effectiveness of IAEA safeguards to the safeguards criteria that are to be considered. For this purpose PNL used the model and computer programs developed as part of Task C.5 (Estimation of Inspection Effort) of the Program of Technica1 Assistance. In order to apply the C.5 model to perform an analysis of the impact of safeguards criteria, a three-step procedure is followed. First, the input variables to the model are systematically varied using an experimental design. Second, the response of the system to changes in the input parameters is calculated and recorded. Third, a standard statistical software package is used to analyze the results.

The example application of the methodology involves low-enriched uranium conversion and fuel fabrication facilities. The effects of variations in seven parameters are considered: false alarm probability, goal probability of detection, detection goal quantity, the plant operator's measurement capability, the inspector's variables measurement capability, the inspector's attributes measurement capability, and annual piant throughput.

Among the key results and conclusions of the anaiysis are the following:

- The variables with the greatest impact on the probability of detection are the inspector's measurement capability, the goal quantity, and the chroughput. 
- The variables with the greatest impact on inspection costs are the throughput, the goal quantity, and the goal probability of detection.

- There are important interactions between variables. That is, the effect of a given variable often depends on the level or value of some other variable. With the methodology used in this study, these interactions can be quantitatively analyzed.

- Reasonabiy good approximate prediction equations can be developed using the methodology described here. 
SUIMARY . . . . . . . . . . . . . . . . . . . . .

INTROOUCTION . . . . . . . . . . . . . . . . . . . . 1

BACKGROUND . . . . . . . . . . . . . . . . . 1

Task C.5, Estimation of Inspection Effort

OBJECTIVES, SCOPE AND TECHNICAL APPROACH . . . . . . . . . 2

Technical Approach . . . . . . . . . . . . 4

Varying the Inputs. . . . . . . . . . 4

Measuring the Response , . . . . . . . . . 6

Analyzing the Results . . . . . . . . . 6

CONTENTS OF THIS REPORT . . . . . . . . . . . . . . . . . . 7

METHODOLOGY . . . . . . . . . . . . . . . . .

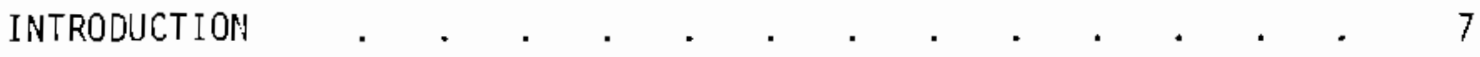

POSSIBLE APPROACHES . . . . . . . . . . . . . . . . . 8

SELECTION OF AN EXPERIMENTAL DESIGN . . . . . . . . . . . . 9

ANALYSIS OF THE DATA . . . . . . . . . . . . . . 10

APPLICATION OF THE METHODOLOGY . . . . . . . . . . . . . . . . 17

APPLICATION OF THE METHOO OF C.5 TO A LEU
CONVERSIOH-FABRICATION PLANT . . . . . . . . . . .

Facility Description . . . . . . . . . . 12

Stratum Descriptions . . . . . . . . . . . 12

Receipts. . . . . . . . . . . 12

Shipments . . . . . . . . . . . . 14

Inventories. . . . . . . . . . . 15

Safeguards Approach . . . . . . . . . . . 23

Inspection Tasks . . . . . . . . . . . 24 
Planning, Preparation and Travel . . . . . . 25

Foliow-up Actions from Previous

Inspections . . . . . . . . . . . 25

Examination of Records . . . . . . . . . 25

Comparison Between Records and Reports . . . . . 28

Recording of Book Inventory . . . . . . . 28

Verification of Inventory Changes and/or

Material Flow . . . . . . . . . . . 28

Verification of Inventory . . . . . . . . . 29

Verification of Quality and Functioning
of Operator's Measurement System . . . . . . .

Installation, Servicing or Verification of

Containment and Surveillance Devices . . . . . 29

Activities in Respect of MUF's, SRD's and

Accidental Losses . . . . . . . . . . . 29

Post-Inspection and Evaluation Activities,

Including Travel . . . . . . . . . . 29

Inspection Plan . . . . . . . . . . . . 30

Estimation of Inspection Effort. . . . . . . . . 46

INPUTS TO THE ANALYSIS . . . . . . . . . . 48

Inputs Pertaining to the Facility Model . . . . . . 48

Stratum Description Information . . . . . . 48

Measurement Error Information . . . . . . . 48

Inputs Pertaining to the Safeguards Goals . . . . . 50

Inputs Pertaining to the Safeguards Approach . . . . 50

SELECTION OF FACTORS OF INTEREST . . . . . . . . . . . . 51

IDENTIFICATION AND SELECTION OF RESPONSES OF 
Responses Related to Effectiveness . . . . . . 53

Responses Related to Efficiency . . . . . . 53

EXPERIMENTAL DESIGN AND DATA GENERATION . . . . . . . . 54

Region of Interest . . . . . . . . . . 54

False Alarm Probability. . . . . . . . 54

Non-detection Probability . . . . . . . . 55

Goai Quantity . . . . . . . . . . . 55

Operator's Measurement Capability . . . . . 55

Inspector's Measurement Capability . . . . . 55

Crossover Point. . . . . . . . . . 58

Plant Size (Annual Throughput) . . . . . . 58

Summary of the Ranges of Input Factors
Considered . . . . . . . . . . . 58

Experimental Design . . . . . . . . . . . 58

ANALYSIS . . . . . . . . . . . . . . . . 64

Setting Up the Data. . . . . . . . . 64

Characteristics of the Box-Behnken

Experimental Design. . . . . . . . . . 65

Behavior of the Response Variables . . . . . . 76

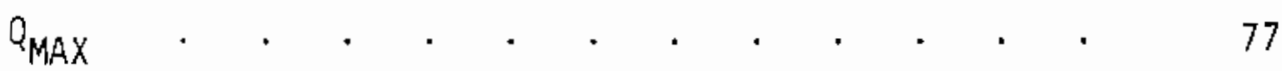

Conclusions Concerning $\mathrm{Q}_{\mathrm{MAX}}$ - . . . . . . . . 88

Total Cost . . . . . . . . . . . . . 89

Conclusions Concerning Total Cost . . . . . . 95 
DISCUSSION OF THE IMPACT OF THE INPUT VARIABLES . . . . . . . 98

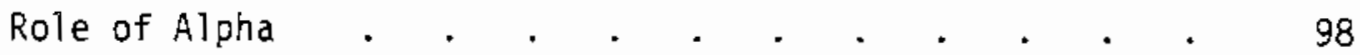

Role of Beta. . . . . . . . . . . . 100

Role of Goal Quantity . . . . . . . . . 101

Role of Operator's Measurement Capability . . . . 102

Role of Inspector's Measurement Capabjlity . . . . 103

Role of Gamma, the Crossover Point . . . . . . 104

Role of Throughput . . . . . . . . . 105

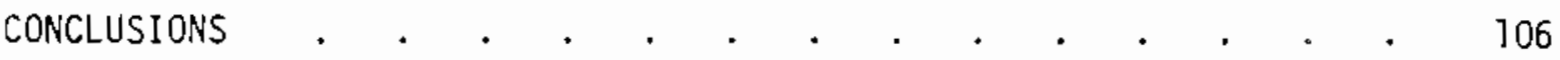

REFERENCES . . . . . . . . . . . . . . . . 107

APPENDIX A - ESTIMATION OF INSPECTION EFFORT . . . . • . . A.I

APPENDIX B - INVESTIGATION OF BOX-BEHNKEN DESIGN INPUT VARIABLES • B.

APPENDIX C - GRAPHS AND ANALYSIS OF RESPONSE VARIABLES . . . . . c. 


\section{FIGURES}

1. Task C.5 - Develop a Method for Estimating Inspection Effort

2. Task C.19 - Apply the Method . . . . . . . . 5

3. Process Flow Diagram . . . . . . . . . . . 13

4. Input Data . . . . . . . . . . . . 49

5. Histogram of Alpha (False Alarm Probability) . . . . 66

6. Histogram of $\times 1 \times 1$ (A1pha Squared) . . . . . . . 68

7. Histogram of $\times 1 \times 2$ (Alpha Times Beta). . . . . . 69

8. Alpha Versus Alpha . . . . . . . . . . 70

9. Beta Versus Alpha . . . . . . . . . . 71

10. Alpha Versus Alpha Squared $(\times 1 \times 1)$. . . . . . . 73

11. Beta Versus Alpha Squared $(X 1 \times 1)$. . . . . . . . 74

12. Beta Squared $(\times 2 \times 2)$ Versus Alpha Squared $(\times 1 \times 1)$. . . 75

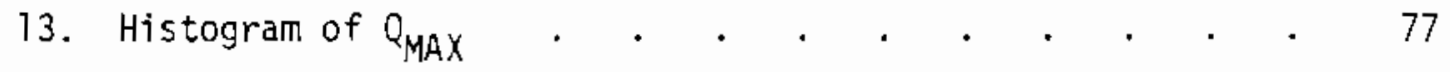

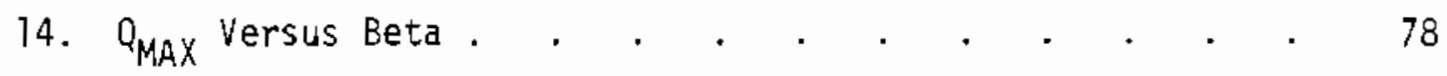

15. Histograms of $Q_{\text {MAX }}$ Versus Alpha . . . . . . . . 79

16. QMAX Versus Inspector's Measurement Capability . . . 81

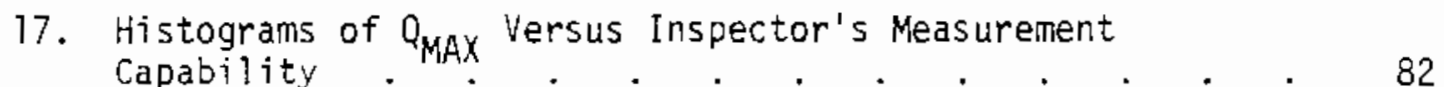

18. Histogram of Total Cost (Man-Days) . . . . . . . 90

19. Total Cost Versus Throughput . . . . . . . . . 91

20. Histograms of Total Cost Versus Throughput . . . . 92

21. Total Cost Versus Goal Quantity $(G Q)$. . . . . . . 93

22. Histograms of Total Cost Versus Goal Quantity (GQ) . . 94 
B.1. Histogram of Beta (Goal Probability of Non-Detection. . B.2

B.2. Histogram of Goal Quantity (GQ) . . . . . . . . B.3

B.3. Histogram of MCAPOP (Operator's Measurement Capability) . B.4

B.4. Histogram of MCAPINSP (Inspector's Measurement Capability) . . . . . . . . . . . . B.5

B.5. Histogram of Gamma (Crossover Point) . . . . . . B.6

B.6. Histogram of Throughput . . . . . . . . . . . B.7

B.7. Histogram of $\times 2 \times 2$ (Beta Squared). . . . . . . B.8

B.8. Histogram of $\times 3 \times 3$ (Goal Quantity Squared) . . . . 3.9

B.9. Histogram of $\times 4 \times 4$ (MCAPOP Squared) . . . . . . B. 10

B.10. Histogram of $\times 5 \times 5$ (MCAPINSP Squared) . . . . . . B.11

B.17. Histogram of $\times 6 \times 6$ (Garma Squared) . . . . . . B. 12

B.12. Histogram of $X 7 \times 7$ (Throughput Squared) . . . . . B.13

B.13. Histogram of $\times 7 \times 3$ (Alpha Times $G Q$ ) . . . . . . . B.14

B.14. Histogram of $X 1 \times 4$ (Alpha Times MCAPOP) . . . . . B.15

B.15. Histogram of XiX5 (A1pha Times MCAPINSP). . . . . B.16

B.16. Histogram of $X 7 \times 6$ (Alpha Times Garma) . . . . . B.17

B.17. Histogram of $\times 1 \times 7$ (A1pha Times Throughput) . . . . B.18

B.18. Goal Quantity Versus Alpha . . . . . . . . . B.19

B.19. Operator's Measurement Capability Versus Alpha . . . B.20

B.20. Inspector's Measurement Capability Versus Alpha . . . B.2?

B.21. Crossover Point Versus Alpha . . . . . . . . B.22

B.22. Throughput Versus Alpha . . . . . . . . . B.23

C.1. Q QAX Versus Goal Quantity . . . . . . . . 0.2

C.2. Histograms of $Q_{\text {MAX }}$ Versus Beta . . . . . . . . c.3 


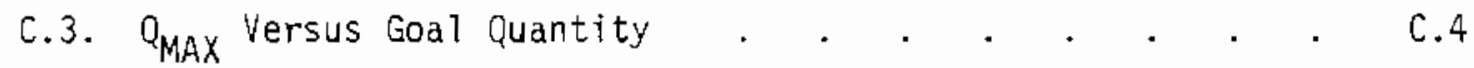

C.4. Histograms of $Q_{\text {MAX }}$ Versus Goal Quantity . . . . . . $c .5$

C.5. Qmax Versus Operator's Measurement Capability . . . . C.6

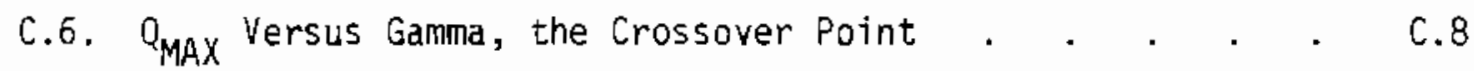

C.7. Q Q $\operatorname{MAX}$ Versus Throughput . . . . . . . . . . C.9

c.8. Histograms of $\mathrm{Q}_{\text {MAX Versus Throughput . . . . . . }}$. .10

C.9. Total Cost Versus Alpha . . . . . . . . . . . c.11

C.10. Histograms of Total Cost Versus Alpha . . . , . C.12

C.11. Total Cost Versus Beta . . . . . . . . . C.13

C.12. Histograms of Total Cost Versus Beta. . . . . . C.14

C.13. Total Cost Versus Operator's Measurement Capability . . C.16

C.14. Histograms of Total Cost Versus Operator's Measurement
Capability . . . . . . . . . . . . . . . . . . . .

C.15. Total Cost Versus Inspector's Measurement Capability . . C.18

C.16. Histograms of Total Cost Versus Inspector's Measurement
Capability . . . . . . . . . . . . . . . . . . .

C.17. Tota] Cost Versus Gamma, the Crossover Point . . . . C.20

C.18. Histograms of Totat Cost Versus Gamma, the Crossover
Point . . . . . . . . . . . . . . . . . . . 


\section{TABLES}

1. Annual Material Balance Data . . . . . . . . . . . 19

2. Notation . . . . . . . . . . . . . . . . 20

3. Detailed Material Balance Data . . . . . . . . . . . 21

4. Data Relative to Measurement Errors . . . . . . . 22

5. Bu1k Measurement Error File . . . . . . . . . 32

6. Sampling Error File . . . . . . . . . . . . . 33

7. Analytica? Error File . . . . . . . . . . . . . . 34

8. Between-Laboratory Components File . . . . . . . . . 35

9. Variance of MUF . . . . . . . . . . . . . . . 37-38

10. Variance of $\hat{D}$. . . . . . . . . . . . . . . . . . . . . . . . . . . .

11. Attributes Sampling Plan . . . . . . . . . . . . 43

12. Variables Sampling Plan . . . . . . . . . . . . . . . 44

13. Effectiveness of Sampling Plans . . . . . . . . . . . 45

14. Total Inspection Effort for Routine Inspections (One-Year . . 47

15. Factors Selected for Detailed Analysis . . . . . . . . 52

16. Ranges of Measurement Error Standard Deviations (Operator). . 56

17. Ranges of Measurement Error Standard Deviations (Inspector) • 57

18. LEU Conversion-Fabrication Plants Under IAEA Safeguards
(November 1979) . . . . . . . . . . . . 59

19. Summary of Ranges of Input Factors . . . . . . . . . . 59

20. Seven Variable Box-Behnken Designs . . . . . . . . . 61

21. Table of Data . . . . . . . . . . . . . . . . 63

22. List of Nineteen Response Variables . . . . . . . . . 76 
23. Summary of Regression Analysis: RMAX . . . . . . . . . . 84

24. Coefficients Involving Inspector's Measurenent Capability . . 85

25. Coefficients Involving Throughput . . . . . . . . . . 85

26. Coefficients Involving Goa1 Quantity . . . . . . . . 86

27. Coefficients Involving the False Alarm Probability, Alpha . . 86

28. Coefficients Involving the Goal Nondetection Probability, Beta . . . . . . . . . . . . 87

29. Coefficients Involving the Operator's Measurement Capability . 87

30. Coefficients Involving the Crossover Point, Gamma . . . . 88

31. Summary of Regression Analysis: Total Cost . . . . . . . . 96

32. Variables That Have the Most Impact on Total Cost . . . . 97

A.1. Routine Inspection Effort for One-Year Period Planning and Preparation . . . . . . . . . . A.3

A.2. Routine Inspection Effort for One-Year Period - Audit . . . A.5

A.3. Routine Inspection Effort for One-Year Period -
Post-Inspection and Evaluation. . . . . . . . . . . . . A.7 
. 
INTROOUCTION

The purpose of Task C.19 of the U. S. Program of Technical Assistance to IAEA Safeguards is to develop and demonstrate a method for analyzing certain quantitative aspects of the cost-effectiveness of safeguards. Specificaily, the task addresses the problem of assessing the impact upon the IAEA resulting from the implementation of specific safeguards technical objectives or criteria. This report describes the method that was deveioped and presents an example application of the method to the case of low-enriched uranium conversion and fuel fabrication facilities.

\section{BACKGROUND}

The Program of Technical Assistance to IAEA Safeguards inciudes two tasks in the general area of inspection effort modeling performed by the Pacific Northwest Laboratory. The first of these was Task C.5, Estimation of Inspection Effort for Chosen Inspection Procedures. Its aim was to develop and demonstrate a cost-effectiveness model that could be used to estimate the manpower required to inspect various types of nuclear facilities as a function of various inspection procedures that might be considered. Task C.5 was completed in July 1979 and is documented in two reports:

M. F. Mullen and M. A. Wincek, Estimation of Inspection Effort, ISP0-35, PNL-2558, Pacific Northwest Laboratory, June 1979.

M. A. Wincek and M. F. Mul Ien, INSPECT - A Packace of Computer Programs for Planning Safeguards Inspections, ISP0-58, PNL-2559, Pacific Northwest Laboratory, Apriz 1979.

The second task in the area of inspection effort modeling - Task C.19, Model for Analysis of the Impact of Safeguards Criteria - is the subject of this report. The objective of Task $\mathrm{C} .19$ is to build upon the results of C.5, using the models developed in C.5 to perform a systematic analys is of the impact of varying certain safeguards criteria. 
Task C.5, Estimation of Inspection Effort for Chosen Inspection Procedures

The objective of Task C.5 was to develop and demonstrate a method for estimating the manpower required to carry out international safeguards inspections of various types of nuclear facilities. The method that was devised for this purpose is shown schematically in Figure 1 . The method requires three kinds of inputs: safeguards objectives or criteria, facility modeis, and a safeguards approach. These inputs jointiy determine a set of inspection activities to be performed by the IAEA. The final step in the method is an evaluation of the IAEA's inspection activities from two points of view: cost of material accounting (or more precisely, man-days of inspection effort) and effectiveness (defined as the probability of detection of the diversion of a specified quantity of nuclear material). The method of 0.5 provides to the safeguards system analyst a systematic way of determining inspection effort and safeguards effectiveness as a function of safeguards goals or criteria, facility models and a safeguards approach.

A noteworthy feature of the method of $C .5$ is the INSPECT package of computer programs for planning and evaluating safeguards inspections. The methodology used in C.5 for evaluating the effectiveness of safeguards is derived from Part $F$ of the IAEA's Safeguards Technical Manual (1) and incorporates a number of computationally difficult procedures and routines. The INSPECT programs heip to make the calculations easier to perform and make it possible to carry out extensive case studies that would be extremely tedious if they had to be done by hand or on a pocket calculator. The INSPECT software is currently operational on the IAEA's computer in Vienna (as well as at PNL) and is being routinely used by both the System Studies Section and the Section for Data Evaluation Services of the Department of Safeguards.

OBJECTIVES, SCOPE AND TECHNICAL APPROACH

The objective of Task $\mathrm{C.19}$ is to show how to apply the cost-effectiveness model developed in Task C.5 to anaiyze the effect on inspection effort and safeguards effectiveness of varying safeguards goals and criteria. 


\section{TASK C.5 - DEVELOP A METHOD}

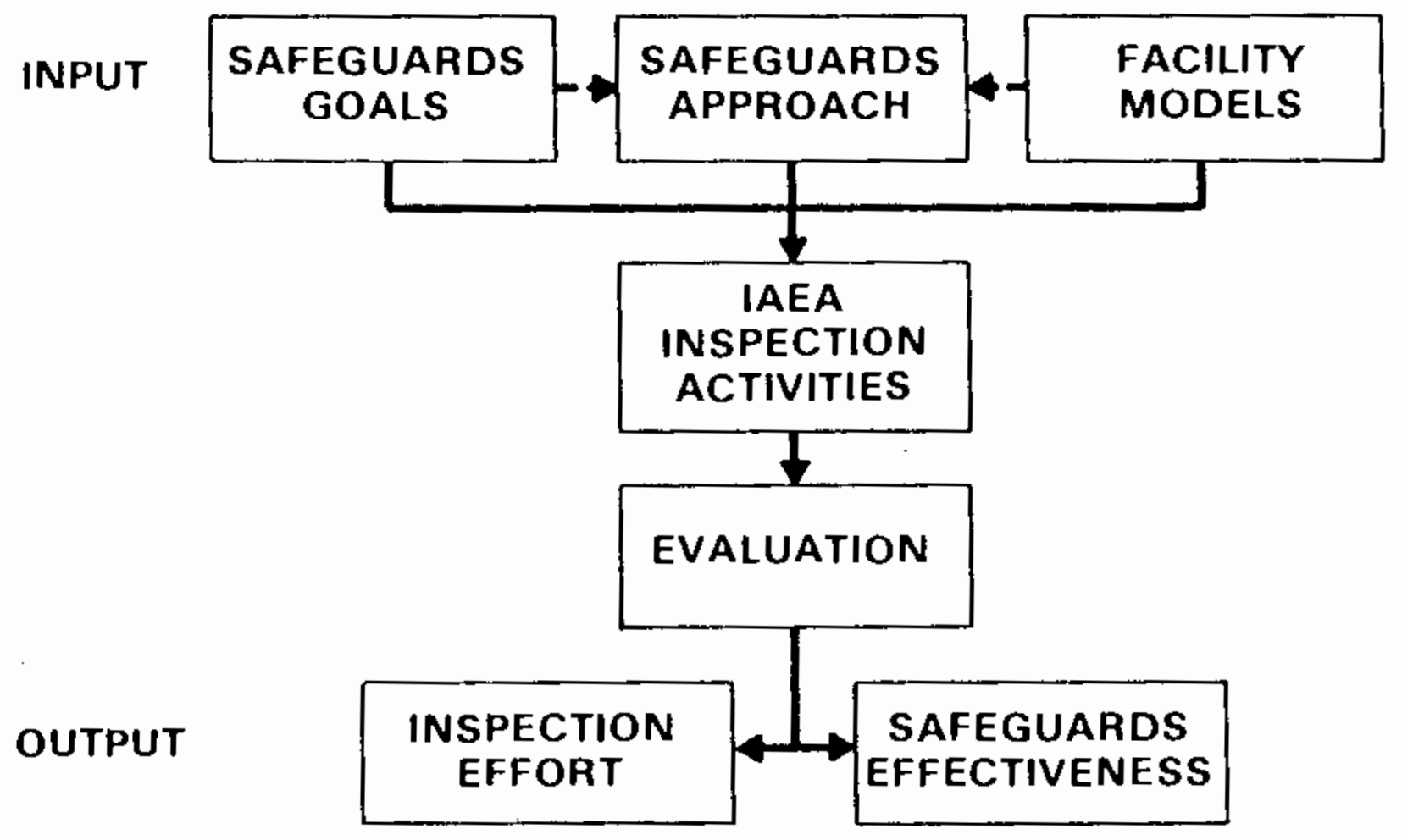

FIGURE 1. Task C.5 - Develop a Method for Estimating Inspection Effort 
To demonstrate the methodology, an anaiysis of safeguards at low-enriched uranium conversion and fuel fabrication facilities is performed.

Technical Approach

The technical approach followed in carrying out Task C.19 is shown schematically in Figure 2. The method developed in Task $C .5$ serves as the starting point of the analysis. The technical approach involves three main steps:

1. Systematically vary the inputs to the model

2. Measure the response of the system to changes in the input parameters

3. Analyze the results

Varying the Inputs

An extensive list of the inputs to the model is generated. The list includes all relevant plant design and operating parameters, safeguards criteria and technical objectives, and key features that characterize the basic safeguards approach. Since the list is very long and not all of the inputs are of imediate interest, the list is screened and a subset of the inputs is selected for detailed analysis. In Task C.19, the following inputs were retained for detailed analysis:

- false aiarm probability

- probability of non-detection

- detection goal quantity

- operator's measurement capability

- inspector's measurement capability

- crossover point (a function of the quality of the inspector's attributes test capability)

- plant throughput

The remaining inputs were examined only qualitatively.

These seven inputs were then varjed according to an experimenta? design (a Box-Behnken design). The use of an experimental design, rather than the simple one-variable-at-a-time approach, is a more efficient and 
TASK C.19 - APPLY THE METHOD: ANALYZE THE IMPACT OF VARYING SAFEGUARDS CRITERIA AND PROCEDURES

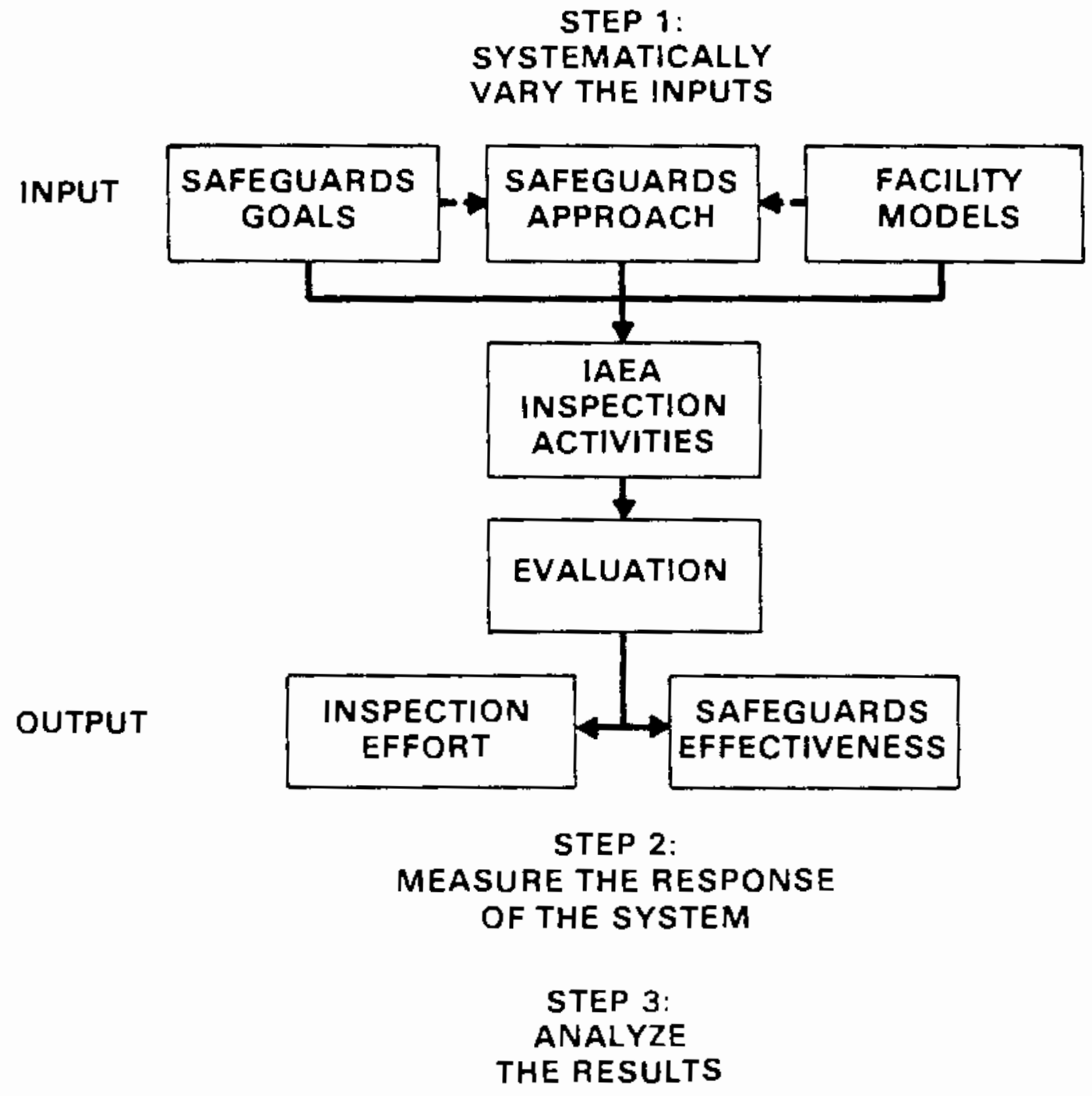

FIGURE 2. Task C.19 - Apply the Method 
effective way of conducting an investigation of this kind.

Measuring the Response

An extensive list of response, or output, variables was generated. It included parameters like $Q_{\text {MAX }}(10)$ (the achieved probability of non-detection assuming the diverter's optimal strategy is employed) the variance of MUF, the variance of $\hat{D}$, the variance of MUF-i inspection effort, and so forth. A few responses were screened out but most were retained, at least initially, because the responses are easy to measure and record -- the INSPECT programs do most of the work.

Each of the cases defined by the experimental design was worked out in detait, using the INSPECT programs and some supplemental analyses and calculations. The results were entered into a data file on the computer.

Analyzing the Results

Two techniques were used to help in interpreting and analyzing the results: graphs of various kinds and regression analysis. Standard computer software was used to facilitate the process. (2)

The graphical methods are straightforward. One simply plots the input variables and the output variables, one versus another.

Regression analysis is used to explore the relationships between variables. Response surfaces or regression equations were fit to the data and studied to help in assessing:

- the sensitivity of the response or output variables to changes in the input factors

- interactions between the factors

- non-linearities in the effect of certain factors

- the relative importance of various factors in determining the behavior of different responses 
CONTENTS OF THIS REPORT

The main body of the report is in three parts. The first part explains the metriodology used in the study. The second contains a detailed account of an example application of the methodology. The application considered involves IAEA safeguards at low-enriched uranium conversion and fuel fabrication plants. The third part contains the discussion and conclusions resulting from the study.

\section{METHODOLOGY}

In this section the methodology used in performing Task c.19 is discussed. The problem is defined in general terms. Several possible approaches for handling the problem are described and compared.

\section{INTROOUCTION}

In Task C.5, we developed a quantitative method that can be used to calculate various parameters related to the efficiency and effectiveness of IAEA safeguards as a function of a certain number of input variables. Formally, the method of $c .5$ defines a functional relationship between a set of input variables and a set of output variables. (In this report, for convenience, we will sometimes call the input variables "factors" and the output variables "responses".) The objective of Task C.T9 is to develop and demonstrate a method for exploring the relationships between these variables in a systematic way.

This method could be used to help answer the following kinds of questions:

- What effect does the goal quantity have on inspection effort?

- What effect does the throughput of a plant have on the effectiveness of safeguards?

- How much incremental inspection effort would be required to change the probability of detection from, say, $90 \%$ to $99 \%$ ?

- What effect do the operator's measurement errors have on the cost and/or effectiveness of safeguards? 
- What effect do the inspector's measurement errors have on the cost and effectiveness of safeguards?

- Are there interactions between factors? For example, in what way does the effect of throughput depend on the level of the operator's or inspector's measurement errors?

- Which factor has the greatest impact on inspection effort?

Which has the greatest impact on effectiveness?

It is clear that the cost-effectiveness model developed in Task 0.5 can provide insights into these questions. In order to use the model effectively, a systematic approach is needed.

\section{POSSIBLE APPROACHES}

In forma? terms, Task C.19 is aimed at studying the behavior or properties of a model. This can be done in several ways.

One approach is to subject the model to logical analysis, working directly with the equations and algorithms to gain insights into the relationships between variables. The disadvantage of this theoretical approach is that the model in question is quite complicated. A great deal of mathematical and statistical machinery is needed, especialiy for quantifying the effectiveness of safeguards.

Instead of analyzing the internal structure of the model, one can take a different tack and simply study some specific cases to see how the model behaves. This "experimental" or case study approach avoids some of the difficulties of the theoretical approach. The results can be presented and understood in a direct and concrete manner. It is not necessary to become too involved in theoretical and mathematical details. But there is also some risk that certain important features of the problem may be overlooked or misinterpreted. In practice, both theory and examples are useful.

The simplest and most intuitive way of working with the case study or experimental approach is to begin with a base case or point of reference. A number of factors are identified whose effect is of interest. To assess the impact of these factors, one factor at a time is varied, with the base case used as the starting point. Each factor is studied separately at 
several levels; all the other factors are kept at their base case levels.

This one-factor-at-a-tine approach is not without merit. Its chief appeal is its simplicity, but it is also a reasonably effective strategy when the response is a complicated function of any given factor, and when the factors act more or less independently of one another.

The disadvantages of the one-factor-at-a-time approach are that the results of the analysis may differ depending on which particular case is selected as the reference, possible interactions between factors are completely ignored, and more efficient ways of obtaining the same information are often avaiiable.

The one-factor-at-a-time approach is an example of an experimental design. Many other kinds of experimental designs are available, most of which allow more than one factor at a time to be varied. Specialized designs have been devised for many applications. In general, these designs have the following advantages over the one-factor-at-a-tine strategy:

- interactions between factors can be seen

- the designs make more efficient use of 1 imited experimental resources

- experimental errors can be estimated

The main disadvantage of the more elaborate experimental designs is that they have a less direct intuitive appeal; it is not always easy to see how or why they work.

In this study, we have rejected the one-factor-at-a-time approach in favor of a specialized experimental design. As we shall show, there are important interactions between the input variables which we would fai? to discover if we used the one-factor-at-a-time approach.

\section{SELECTION OF AN EXPERIMENTAL DESIGN}

The choice of a particular experimental strategy depends on the objectives sought. In this study, we are interested in the effects of the factors and in possible interactions between factors. We know from theoretical considerations and from prior studies that there are important non-linearities. We are interested in exploring the possibility of developing a prediction equation so that we could calculate approximately the values 
of various responses (for example, inspection effort) as a function of some key input parameters without having to carry out a detailed, facility-specific analysis.

To achieve these objectives, we would Tike to fit the parameters of a second order polynomial in the input factors. We are hypothesizing that a second order polynomial, with linear terms, squared terms, and interaction or cross product terms, can provide a reasonably good approximation to the exact model. (One can regard it as a second order Taylor series approximation.)

A variety of experimental designs is available for these purposes. (3) We have chosen to use a Box-Behnken design (4) for the sake of convenience; it is a three-level design, which simplifies matters in an "administrative" sense. Some alternative designs involving more than three levels have certain relatively minor advantages but are somewhat more difficult to work with.

The Box-Behnken designs comprise a subset of the full three level factorial design. (The three level factorial consists of al 1 possible combinations of three possible levels of each factor; for $k$ factors, the three level factorial consists of $3^{k}$ cases.) Their geometry is such that good estimates of the parameters of a second order polynomial can be obtained. The prediction variance tends to be fairly constant across the experimental region and there are enough "extra" points that experimental error or, as in this study, lack of fit can be assessed.

ANALYSIS OF THE DATA

The main tool in the formal analysis of the results of the experiment is regression analysis (fitting equations to data). There are many excellent references on this subject, so we do not discuss it here. Much has aiso been written on the pitfalls involved. It should be noted that we are dealing in this study with a designed experiment so that the data have special properties. For example, we shall see that all the input values are uncorrelated. Another unusual aspect of the data considered here is that the experimental error is zero - if the same case is run twice, exactly the same responses will occur - so that all residuals in the regression analysis are due to lack of fit. 
In studying the relationships between responses and input variables, we look at scatter plots of responses versus inputs. This is not always a good idea, particularly when there are correlations in the input data. However, in this case because of the experimental design, the graphs do show something meaningful about the data. They provide some visual aid in examining the regression equations.

\section{APPLICATION OF THE METHODOLOGY}

In this section, we demonstrate how the methodology described earlier can be applied to low-enriched uranium (LEU) conversion and fuel fabrication plants. First, we show how the method of C.5 is used for LEU conversionfabrication plants. We identify the input variables or factors that enter into the analysis. These factors are then discussed briefly and a subset is chosen for detailed study. We then identify and discuss briefiy the output variables or responses to be considered. Next, we describe the experimental design used to vary the inputs systematically and we explain how the design is used to generate the data to be analyzed. Next, we analyze the data by graphical and response surface methods. Finally, we present some conclusions and recommendations based on the study. APPLICATION OF THE METHOO OF C.5 TO A LEU CONVERSION-FABRICATION PLANT

The method developed in Task C.5 is the basic tool to be used in analyzing the impact of safeguards criteria. In this section, we review briefly the method of $C .5$ to establish the baseline for the subsequent detailed analyses of safeguards criteria.

The case presented here is a low-enriched uranium conversion-fabrication plant with a throughput of 400 tons of uranjum per year and an inventory (at the time of physical inventory taking) of approximately 134 tons (inventory $\cong 34 \%$ of annual throughput). The assumed measurement errors are consistent with international standards of measurement accuracy, which at uranium fabrication plants specify that the expected accuracy of a material balance (expressed as the standard deviation of MUF) should be $0.3 \%$ of the throughput. $\{5)$ 
The method of 0.5 has three main steps:

- define the facility

- outline the material accounting safeguards approach

- estimate the required inspection effort and evaluate the effectiveness of the safeguards approach.

These three topics are discussed in more depth in the next three sections.

\section{Facility Description}

The plant takes $3 \%$ enriched $\mathrm{UF}_{6}$ as the feed material and converts it via the anmonium diuranate process to $\mathrm{VO}_{2}$ powder. The $\mathrm{UO}_{2}$ powder is used to manufacture pellets. These are loaded into fuel rods which are assembled into fuel assemblies. The plant has an internal scrap recovery process. A process flow diagram appears in Figure 3. Associated with each plant material type is a set of random and systematic measurement errors. These are broken into three types: weighing, sampling, and analysis. of ten the information on sampling errors is not well known, and it is lumped in with analysis errors.

\section{Stratum Descriptions}

The model plant has nineteen strata, which are described below.

Receipts. There is one input stratum: cylinders of $U_{6}$. The annual input is 276 cylinders. Each cylinder is weighed, three samples are taken and each sample is analyzed once. The average element weight is $1,450 \mathrm{~kg}$. The measurement error standard deviations are as follows.

\section{Relative Error Standard Deviations \\ $\mathrm{UF}_{6}$ Cylinders}

$\begin{array}{lll} & \frac{\text { Random }}{.00133} & \text { Systematjc } \\ \text { Weighing } & .00163 \\ \text { Sampling } & .0017 & .00144 \\ \text { Analysis } & .00148 & .00140\end{array}$




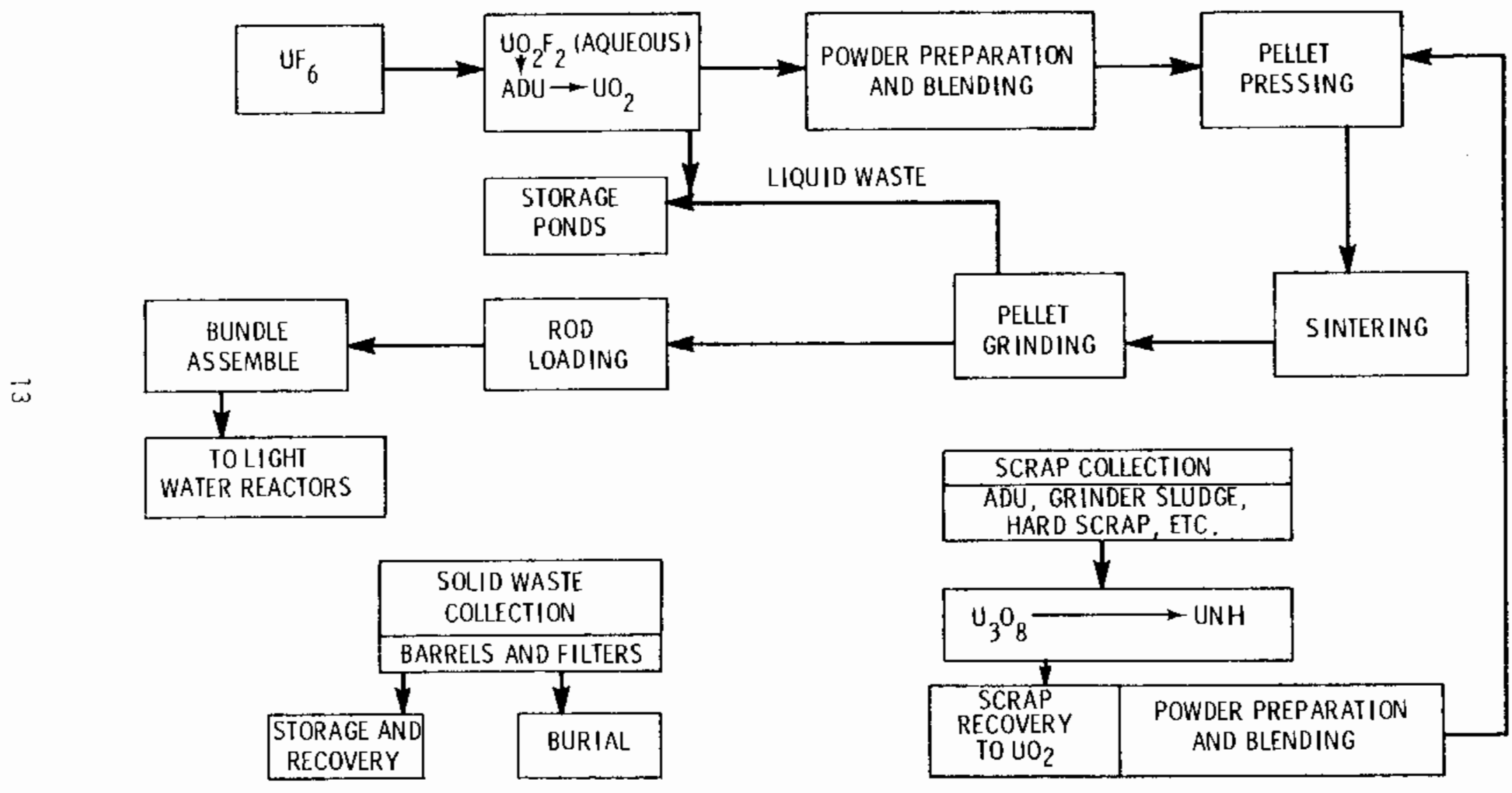

FIGURE 3. Process Flow Diagram 
Shipments. There are two output strata: $\mathrm{VO}_{2}$ fuel rods and measured discards (waste). The operator's fuel rod measured values are based on measurements made at the pellet loading station. (The fuel assemblies do not appear in the material balance; they are assembled and stored under item control.)

The average fuel rod element weight is $2.9 \mathrm{~kg}$, and there are $i 3$ rods per batch. For the 400 ton throughput facility, there are 2170 batches per year, for a total of 136,710 fuel rods per year. The average element factor for each batch is assumed to be based on the results of three samples, with one analytical determination per sample. The following measurement errors are postulated:

Relative Error Standard Deviations

$\mathrm{UO}_{2}$ Fuel Rods

$\begin{array}{llc} & \frac{\text { Random }}{.00083} & \frac{\text { Systematic }}{.00083} \\ \text { Weighing } & \text { Samping and } & \\ \text { Analysis } & .00120 & .00120\end{array}$

The measured discard stratum contains 7,482 items. Each item is a batch. The average item weight is $0.5 \mathrm{~kg}$ of uranium. The operator measures each item non-destructively, with measurement error standard deviations as follows:

ReTative Error Standard Deviations Measured Discards

NDA $\quad \frac{\text { Random }}{.0875} \frac{\text { Systematic }}{.05625}$


Inventories. We have assumed that the beginning and ending inventories are similar in comosition. Each consists of eight strata:

- $u_{6}$ cylinders

- $\mathrm{UO}_{2}$ powder

- $\mathrm{UO}_{2}$ pellets

- $\mathrm{U}_{3} \mathrm{O}_{8}$ powder

- Scrap

- Waste

- Miscellaneous

- Fuel Rods

The $U_{6}$ stratum consists of 40 items. Each item is a batch. The average element weight per item is $1,450 \mathrm{~kg}$. The operator takes three samples per batch and anaiyzes each once. The measurement error data are the same as for the $U_{6}$ stratum in receipts.

The $\mathrm{UO}_{2}$ powder stratum has 66 batches containing 30 items each. The average item contains $13 \mathrm{~kg}$ of urarium. The uranium concentration is established on the basis of two samples per batch, one analys is per sample. The measurement error standard deviations are as follows:

$$
\begin{aligned}
& \text { Relative Error Standard Deviations } \\
& \qquad \mathrm{UO}_{2} \text { Powder }
\end{aligned}
$$

$\begin{array}{lcc}\text { Weighing } & \frac{\text { Random }}{.00113} & \frac{\text { Systematic }}{.00113} \\ \begin{array}{l}\text { Sampling and } \\ \text { Anatysis }\end{array} & .00128 & .0012\end{array}$


The $\mathrm{UO}_{2}$ pellet stratum consists of trays of pellets. Each tray is an $i$ tem. There are 17 batches with 30 items per batch. The average uranium content of an item is $13 \mathrm{~kg}$. The uranium factor for each batch is based on three samples, one analysis per sample. The following relative error standard deviations are assumed:

$$
\begin{aligned}
& \text { Relative Error Standard Deviations } \\
& \mathrm{UO}_{2} \text { Pellet Trays }
\end{aligned}
$$

$\begin{array}{lcc}\text { Weighing } & \frac{\text { Random }}{.00113} & \text { Systematic } \\ \begin{array}{c}\text { Sampling and } \\ \text { Analysis }\end{array} & .000113 & .00098 \\ & & \end{array}$

The $\mathrm{U}_{3} \mathrm{O}_{8}$ Powder stratum consists of buckets containing an average of $20 \mathrm{~kg}$ uranium. There are five batches with 20 items per batch. The uranium concentration is based on two samples per batch with one analysis per sample. The following measurement error standard deviations are assumed:

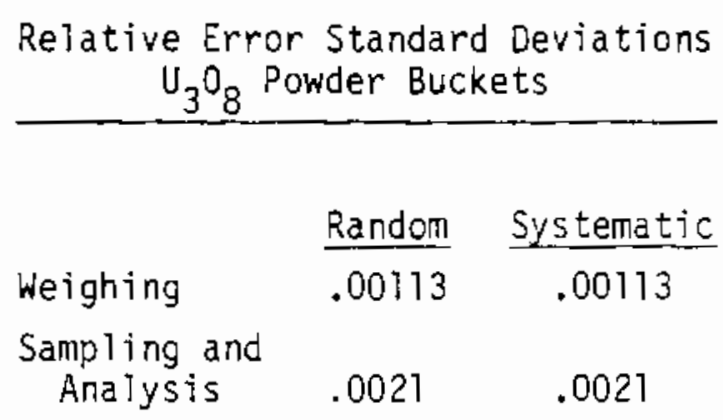

The scrap stratum contains $10,000 \mathrm{~kg}$ uranium in various forms. The average item is assumed to contain $10 \mathrm{~kg}$ uranium. There are 20 batches with 50 items per batch. The batch element factor is based on two samples per batch and one analysis per sample. The relative error standard deviations are as follows: 


\section{Relative Error Standard Deviations}

Scrap

$\begin{array}{lll}\text { Weighing } & \frac{\text { Random }}{.002} & \frac{\text { Systematic }}{.00163} \\ \text { Sampring and } & .02 & .0163 \\ \text { Anarysis } & & \end{array}$

The inventory includes $250 \mathrm{~kg}$ uranium in the form of waste. There are 500 batches with one item per batch. The average item contains $0.5 \mathrm{~kg}$ uranium. The operator measures each item non-destructively, wi th measurement error standard deviations as follows:

$\frac{\text { Reiative Error Standard Deviations }}{\text { Waste }}$

The stratum identified as miscellaneous is a sort of a catch-all that covers the rest of the material on inventory and laboratory sampies. It is assumed that items in this stratum contain an average of $4 \mathrm{~kg}$ uranium. There are 250 batches and one item per batch. The uranium concentration is based on two samples per batch, one analysis per sample. The following measurement errors are assumed:

\section{Relative Error Standard Deviations Miscellaneous}

\begin{tabular}{|c|c|c|}
\hline & Random & Systematic \\
\hline Weighing & .00113 & .0009 \\
\hline $\begin{array}{l}\text { Sampling and } \\
\text { Analys is }\end{array}$ & .00182 & .00163 \\
\hline
\end{tabular}

The fuel rod stratum contains $5,846 \mathrm{~kg}$ uranium. It consists of 32 batches, with 63 items (fuel rods) per batch. Each item contain $2.9 \mathrm{~kg}$ uranium. The measurement error data are the same as for the fuei rod 
stratum in shipments.

The basic material balance data are summarized in Table 1. The detailed material balance data required as input to the INSPECT programs are shown in Tables 2 and 3 . Table 2 explains the notation used in Table 3.

Data relative to measurement errors are summarized in Table 4 . 
TABLE 1. Annual Material Balance Data

Average

Itern Weight

$(\mathrm{kg} U)$

Receipts

$U_{6}$ Cylinders

1,450

Shipments

Fuel Rods

Waste

Inventories

$\mathrm{UF}_{6}$ Cylinders
$\mathrm{UO}_{2}$ Powder
$\mathrm{UO}_{2}$ Pellets
$\mathrm{U}_{3} \mathrm{O}_{8}$ Powder
Scrap
Waste
Miscellaneous
Fuel Rods

276

400,200

2.9

0.5

Number

of Items

Tota 1

Stratum Weight

$(\mathrm{kg} \mathrm{U})$

396,459

3,741
1,450

13

13

20

10

0.5

4

2.9

40
1,980
1,020
200
2,000
1,000
500
4,032

40

,980

200

, 000

, 000

, 032
58,000

25,740

13,260

4,000

20,000

500

2,000

$11,692.8$ 


\section{TABLE 2. Notation}

NOTATION:

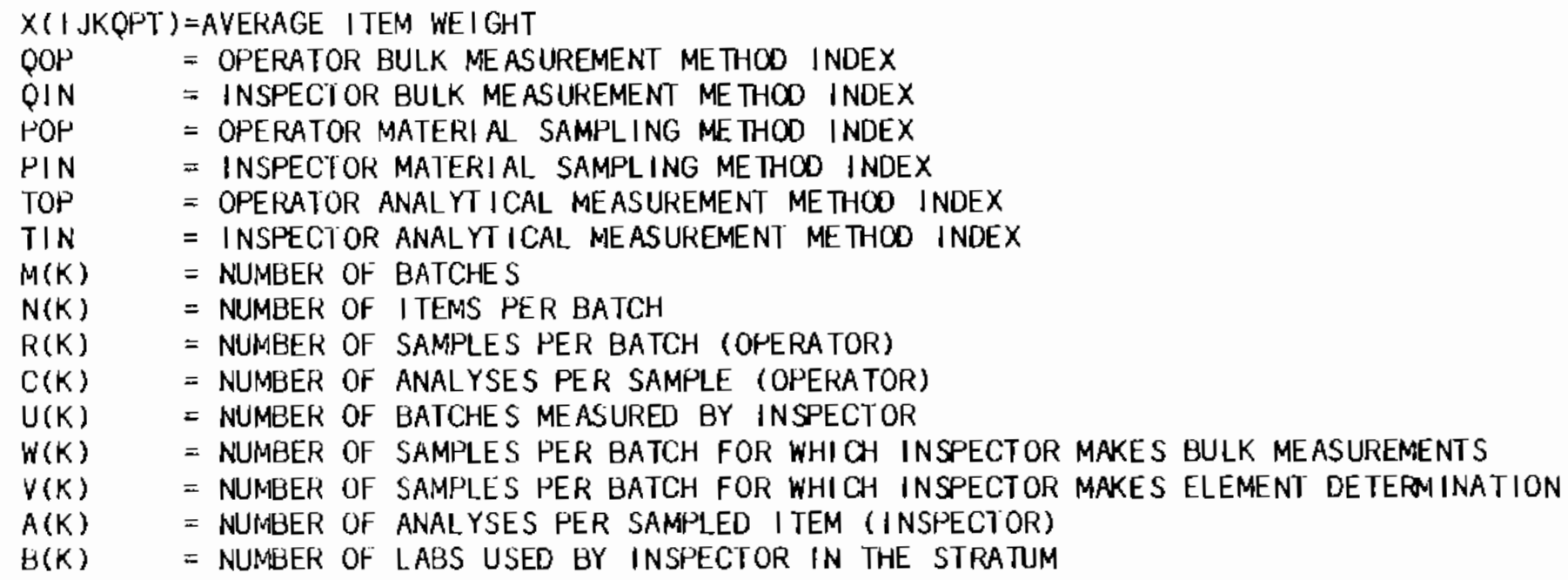

ERROR TABLE DATA IS OECIMAL FRACTION, NOT PERCENT 
TABLE 3. Detailed Material Balance Data

400 IOW HLNN 35.3\$ INYENTORY LEU

HEGINBSHAS INYENTOKY

\begin{tabular}{|c|c|c|c|c|c|c|c|c|c|c|c|c|c|c|c|c|c|}
\hline $\begin{array}{l}\text { SIRATUM NNAE } \\
\text { USHECIOR LAE I }\end{array}$ & $\begin{array}{l}\text { RND } \\
\text { INUEX }\end{array}$ & $x(1,3 \times 0+1)$ & Wor & QIN & rot & HIN & ror & IIN & $M(K)$ & $N(K)$ & $R(K)$ & $C(k)$ & $u(K)$ & $m(x)$ & $Y(k)$ & $A(k)$ & $B(K)$ \\
\hline Ut 6 CYL I NDEER & RS & 1450.000 & 1 & 20 & 1 & 19 & 1 & 19 & 40 & 3 & 3 & 1 & 1 & 1 & 1 & 1 & 1 \\
\hline $\begin{array}{l}022 \text { rowluth } \\
10000\end{array}$ & (4) (b) & 13.0000 & 2 & 21 & $z$ & 20 & 2 & 20 & ob & su & 2 & 1 & 1 & 1 & 1 & 1 & 1 \\
\hline VO2 MELLETS & 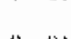 & 15.600 & 5 & 22 & 5 & 21 & $y$ & 21 & 34 & 30 & 5 & 1 & 1 & 1 & $l$ & 1 & 1 \\
\hline $\begin{array}{l}\text { USOE POHOEK } \\
100 \\
100\end{array}$ & (1) us & 20.000 & 4 & 25 & 4 & 22 & 4 & 22 & 10 & 20 & 2 & 1 & 1 & $\mathbf{I}$ & 1 & 1 & 1 \\
\hline $\begin{array}{lll}\text { SORAH } & \\
10 & 0 & 0\end{array}$ & $\begin{array}{ll}0 & 0\end{array}$ & 10.000 & $b$ & 24 & b & 23 & $b$ & 23 & 40 & 50 & 2 & 1 & 1 & i & 1 & 1 & 1 \\
\hline $\begin{array}{llll}\text { WASTE } & 0 & 0 & 0\end{array}$ & $\begin{array}{ll}0 & 01\end{array}$ & 0.500 & 0 & $2^{5}$ & 0 & 24 & 6 & 24 & 1000 & 1 & 1 & 1 & 1 & 1 & 1 & 1 & 1 \\
\hline $\begin{array}{l}\text { MI SCEL LANE OUS } \\
10000\end{array}$ & $\begin{array}{lll}\text { Us } & \\
0 & \text { in }\end{array}$ & 4.000 & 7 & 26 & 1 & $z$ & 1 & $2 b$ & 304 & 1 & 2 & 1 & 1 & 1 & 1 & 1 & 1 \\
\hline $\begin{array}{l}\text { twet HOUS } \\
1\end{array}$ & $0 \quad u$ & 7.500 & 0 & 21 & 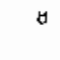 & 20 & 8 & 26 & 0.4 & is & g & 1 & 1 & 1 & 1 & 1 & 1 \\
\hline & & & & & & EIMIS & & & & & & & & & & & \\
\hline $\begin{array}{l}\text { SiRATUM NAHA } \\
\text { HNSHCCTOK LAES }\end{array}$ & $\begin{array}{l}A N Q \\
\text { INOt } X\end{array}$ & $x(1)=\{s=1)$ & ox & PiN & $r(x$ & rIN & $10+$ & $\pi \mathrm{N}$ & $M(K)$ & $N(K)$ & $k(k)$ & $c(k)$ & $v(k)$ & $w(K)$ & $v(k)$ & $A(K)$ & $t(k)$ \\
\hline Ut 6 CYL INDEN & & 1430.6000 & $\dot{y}$ & 24 & ' & 26 & y & $2 \mathrm{~b}$ & 270 & 1 & 3 & ' & $i$ & 1 & 1 & 1 & 1 \\
\hline & & & & & & $F+N=1$ & & & & & & & & & & & \\
\hline $\begin{array}{l}\text { УERATUK NAME } \\
\text { INS̈HECTOH LAE OH }\end{array}$ & $\begin{array}{l}\text { AND) } \\
\text { iNDLEX }\end{array}$ & $x(1) \pi(k)-1)$ & vor & vis & $h a$ & $\operatorname{HIN}$ & 100 & $\| N$ & $M(K)$ & $N(K)$ & $k(k)$ & $c(K)$ & $v(k)$ & $w(k)$ & $v(k)$ & $A(x)$ & $B(K)$ \\
\hline $\begin{array}{l}\text { kuos } \\
10\end{array}$ & $\left(\begin{array}{ll}1 & 0\end{array}\right)$ & 2,900 & $i 1$ & so & 10 & $2 y$ & 10 & $x$ & $21 \%$ & 03 & s & 1 & $i$ & 1 & 1 & 1 & 1 \\
\hline $\begin{array}{cccc}\text { WASIE } \\
1 & 0 & 0 & 0\end{array}$ & $\begin{array}{ll}0 & 0\end{array}$ & 0.560 & 12 & 31 & 11 & so & 11 & s0) & 7462 & 1 & 1 & 1 & 1 & 1 & 1 & 1 & 1 \\
\hline & & & & END & ING I & WENIC & & & & & & & & & & & \\
\hline 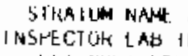 & $\begin{array}{l}\text { ANDE } \\
\text { TAUEX }\end{array}$ & $x(1 \mathrm{JK}(1) 1)$ & wo & $Q \mathbb{N}$ & rot & $r k s$ & Tot & ItN & $M(K)$ & $N(K)$ & $k(k)$ & $c(k)$ & $U(k)$ & $w(K)$ & $r(k)$ & $A(k)$ & $b(k)$ \\
\hline $\begin{array}{l}\text { St D CYLINUEH } \\
1000\end{array}$ & $\begin{array}{rl}H 5 & 01 \\
0 & 41\end{array}$ & 1450.060 & y & 32 & $y$ & 31 & y & 31 & Ao & 1 & 3 & 1 & 1 & 1 & 1 & 1 & $i$ \\
\hline 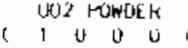 & & 15.060 & 13 & 39 & 12 & 92 & 12 & $s t$ & 66 & 30 & 2 & 1 & 1 & 1 & 1 & 1 & 1 \\
\hline WOZ rELLEES & $\begin{array}{ll}0 & 0\end{array}$ & 13.000 & 14 & 34 & 15 & 33 & 13 & 33 & 34 & so & 5 & 1 & 1 & 1 & 1 & 1 & 1 \\
\hline $\begin{array}{l}13 \times 86 \text { rowotik } \\
1000\end{array}$ & $\begin{array}{ll}0 & 0\end{array}$ & 20.000 & b) & s' & 14 & 34 & 14 & 34 & 10 & 20 & 2 & 1 & 1 & 1 & $t$ & 1 & 1 \\
\hline $\begin{array}{llll}\text { sedes } & & \\
1 & 4 & 0 & 0\end{array}$ & if (u) & 10.016 & 16 & 36 & 15 & sb & is & 35 & 40 & 30 & 2 & $i$ & 1 & 1 & 1 & 1 & 1 \\
\hline $\begin{array}{lll}\text { WASIE } & 0 \\
1 & 0 & 0\end{array}$ & (1) (b) & 0.500, & 13 & 97 & 10 & 36 & 16 & 36 & 1000 & 1 & 1 & 1 & 1 & I & 1 & 1 & 1 \\
\hline $\begin{array}{l}\text { MI SCLE L LAEGWUS } \\
10000\end{array}$ & Js & $4.600 t$ & 16 & 30 & it & st & 12 & si & $\$ 00$ & 1 & 2 & 1 & 1 & 1 & 1 & 1 & 1 \\
\hline L ROOS & & 2.200 & $1 y$ & 39 & 10 & $5 \theta$ & 16 & 34 & 64 & 61 & 3 & $i$ & 1 & 1 & 1 & 1 & 1 \\
\hline
\end{tabular}


TABLE 4. Data Relative to Measurement Errors

\begin{tabular}{|c|c|c|}
\hline Material Iype & $\begin{array}{l}\text { Random Error } \\
\text { Relative Standard Deviation }\end{array}$ & $\begin{array}{c}\text { Systematic Error } \\
\text { Relative Standard Deviation }\end{array}$ \\
\hline \multicolumn{3}{|l|}{$U F_{6}[y]$ inders } \\
\hline $\begin{array}{l}\text { Weighing } \\
\text { Sampling } \\
\text { Analys is }\end{array}$ & $\begin{array}{l}.00133 \\
.0017 \\
.00148\end{array}$ & $\begin{array}{l}.00163 \\
.00144 \\
.00140\end{array}$ \\
\hline \multicolumn{3}{|l|}{$\mathrm{UO}_{2}$ Fuel Rods } \\
\hline $\begin{array}{l}\text { Weighing } \\
\text { Sampling and } \\
\text { Analysis }\end{array}$ & $\begin{array}{l}.00083 \\
.00120\end{array}$ & $\begin{array}{l}.00083 \\
.00120\end{array}$ \\
\hline \multicolumn{3}{|c|}{ Measured Discards } \\
\hline NOA & .0875 & $.0 \$ 625$ \\
\hline \multicolumn{3}{|l|}{$\mathrm{UO}_{2}$ Powder } \\
\hline $\begin{array}{l}\text { Weighing } \\
\text { Sampling and } \\
\text { Analysis }\end{array}$ & $\begin{array}{l}.00113 \\
.00128\end{array}$ & $\begin{array}{l}.00113 \\
.0012\end{array}$ \\
\hline \multicolumn{3}{|l|}{$\mathrm{NO}_{2}$ Pellets } \\
\hline $\begin{array}{l}\text { Weighing } \\
\text { Sampling and } \\
\text { Analysis }\end{array}$ & $\begin{array}{l}.00113 \\
.0012\end{array}$ & $\begin{array}{l}.0009 \\
.00098\end{array}$ \\
\hline \multicolumn{3}{|l|}{$\mathrm{U}_{3} \mathrm{O}_{8}$ Powder } \\
\hline $\begin{array}{l}\text { Weighing } \\
\text { Sampling and } \\
\text { Analys is }\end{array}$ & $\begin{array}{l}.00113 \\
.0021\end{array}$ & $\begin{array}{l}.00113 \\
.0021\end{array}$ \\
\hline \multicolumn{3}{|l|}{ Scrap } \\
\hline $\begin{array}{l}\text { Weighing } \\
\text { Sampling and } \\
\text { Analysis }\end{array}$ & $\begin{array}{l}.002 \\
.02\end{array}$ & $\begin{array}{l}.00163 \\
.0163\end{array}$ \\
\hline \multicolumn{3}{|l|}{ Waste } \\
\hline NDA & .0875 & .05525 \\
\hline \multicolumn{3}{|l|}{ Yiscellaneous } \\
\hline $\begin{array}{l}\text { Weigning } \\
\text { Sampling and } \\
\text { Anatysis }\end{array}$ & $\begin{array}{l}.00113 \\
.00182\end{array}$ & $\begin{array}{l}.0009 \\
.00163\end{array}$ \\
\hline
\end{tabular}


Safeguards Approach

The principal safeguards measure in the model plant is materials accounting. The scope of application of containment and surveillance is assumed to be limited. Briefly, the plant is structured as follows:

- Two material balance areas (MBA's):

1. Shipper/receiver difference area (MBA 1)

2. Process MBA containing LEU; a 11 material in the plant is less than 5\% enriched (MBA2).

- Five flow key measurement points (flow KMP's):

1. Receipts of LEU into MBAT

2. Shipments of LEU from MBAT to a Tocation other than

MBA2 and ail other decreases of the inventory of MBAI

except transfers to MBA2

3. Transfers from MBA1 to MBA2 and all other increases of the inventory of MBA2

4. Snipments from MBA2 and all other decreases of the inventory of MBA2 except measured discards

5. Measured discards

- Inventory key measurement points include:

A. All nuclear material kept on the basis of shipper's measurements

B. $\mathrm{UF}_{6}$ cylinder

C. $\mathrm{UO}_{2}$ powder

D. $\mathrm{UO}_{2}$ pellets

E. $\mathrm{U}_{3} \mathrm{O}_{8}$ powder

F. Scrap

G. Waste

$H$. Fuel rods

I. Fuel assemblies

J. Miscellaneous 
The frequency of physical inventory verification for the 400 ton/year plant was assumed to be twice per year. In practice, the actual frequency might be either once or twice per year, depending on the circumstances. The detection time for low-enriched uranium is in the order of one year. Under the circumstances, the Agency has severai options:

- To verify pellet stacks at the rod-loading stage

- To verify fuel rods before assembly. This can be done in at least two ways. One possibility is to use the SAM-II portable NDA device to verify enrichment and active fuel length. Another possibility is to use the facility's rod scanner (if the facility has one). For verification purposes, the rod scanner must be calibrated using independent standards.

- To verify fue? assemblies in an essentially qualitative manner using the SAM-II. (Instruments now under development may in the future permit a quantitative verification of fuel assemblies.)

Each method closes the material accounting balance area at a different step in the process. Ideally, accurate measurements could be made on finished fuel assemblies, and all diversion paths up to the assemblies would be covered. This technique (active neutron collar) is being developed, and is a few years away. If the material accounting balance area is closed at the finished fuel rod station, adequate measurement techniques exist to verify the amount of nuclear material. Unfortunately, this opens up diversion paths involving rod substitution prior to assembly. The best measurement capability exists on pellets. While this is the highest quality measurement, even more diversion paths exist, as material could be substituted in the rods prior to sealing, as well as rod substitution prior to assembly. For the purpose of illustrating the method of C.5, we assume here that the safeguards approach involves use of the facility's rod scanner to verify fuel rods before assembly.

Inspection Tasks

The activities involved in routine inspections can be divided into the following tasks: 
- Planning, preparation and travel

- Follow-up actions from previous inspections

- Examination of records

- Comparison between records and reports

- Verification of inventory changes and/or material flow

- Verification of inventory

- Verification of the quality and functioning of the operator's measurement system

- Installation, servicing, and/or verification of containment and surveillance devices

- Activities in respect of MUF's, correction of data, SRD's and accidental losses

- Post-inspection and evaluation activities, including travel.

Planning, Preparation and Travel. Planning, preparation and travel includes such activities as: review of background documents (design information, facility attachments, previous inspection reports and working papers, etc.); collection and review of reports for the material balance period; specify technical objectives for the inspection; determine a plan for examining records and reports; determine a preliminary sampling plan for verification of material; plan for the verification and maintenance of containment and surveillance devices (in this case, seals); work out the logistics of the inspection (scheduling, notification, travel arrangements, coordinate inspection team, acquire the necessary equipment); travel to the facility; protocol and preliminary activities at the facility.

Follow-up Actions from Previous Inspections. Discrepancies found in previous inspections may not have been resolved immediately. In this case, follow-up actions may be needed in subsequent inspections to investigate the discrepancies further.

Examination of Records. The objective of the records examination is to confirm the completeness, formal correctness and internal consistency of the records. The records examination, in conjunction with other inspection procedures, is necessary to establish the technical conclusion of the Agency's verification activities, namely, a "statement, in respect of each material 
balance area, of the amount of material unaccounted for over a specific period, giving the limits of accuracy of the amounts stated."

INFCIRC/153 ${ }^{(6)}$ distinguishes between two kinds of records: accounting records and operating records. Accounting records are those records needed to establish a material balance; they contain information on inventories of nuclear material and on inventory changes. Operating records provide supporting information on the accounting records.

The key elements of the accounting records are:

- inventory changes (receipts, shipments, transfers between MBA's, exemptions and de-exemptions, accidental losses, measured discards)

- measurement results used for determination of the physical inventory (weighing, sampling, analysis, item identification and counting)

- adjustments and corrections (shipper/receiver differences, material unaccounted for, correction of mistakes, results from an improved measurement)

The operating records in a low-enriched uranium fuel fabrication plant typically include:

- data from the rod-loading station (as required to establish the content of fuel rods)

- information on accidental losses of material (if any occur)

- detailed information on measurements used in establishing inventories and inventory changes

- detailed information on measurement methods and procedures (calibrations, standards, accuracy, control program, etc.)

- procedures for physical inventory taking; results obtained from physical inventory taking

- procedures for ascertaining the cause and magnitude of any accidental or unmeasured 10s5; results of such investigations

The accounting records typically consist of three basic kinds of records: source documents (also referred to as supporting records or posting documents), 
journals and ledgers. (a)

Source documents are used to make entries in the accounting system; they are the "documents of original entry." Journals provide a chronological record of transactions. Periodically, the entries in the journal are totaled and the totais are posted to the ledgers. Thus, the journals are intermediate between the source documents and the ledgers. Every entry in the journals includes a reference to the source document on which it is based. Ledgers are "books of final entry"; they summarize the status of nuclear material.

There is presently no standard procedure for conducting records examinations. The procedure described here is one possible approach.

The records examination includes the following basic tasks:

- check the agreement between the beginning inventory for the material balance period and the verified ending inventory for the previous period.

- check all receipts against shipper's data

- check transfers between MBA's within the facility

- confirm that records entries are consistent with supporting evidence (operating records, etc.)

- check source documents for completeness and correctness and ascertain whether they have been correctly entered in journals and ledgers

- compare records of shipments against receiver's data

- examine records of all discards, exemptions, de-exemptions and accidenta] losses

- examine all adjustments and corrections

- test the internal consistency of the accounting records and the correctness of the calculations by establishing a trial balance

- check the consistency of accounting records with operating records.

(a) Unfortunately, there is no standard nomenclature for accounting records. The terms used here are common but are not universally accepted. 
Comparison Between Records and Reports. Under INFCIRC/153, the Agency is provided with two kinds of reports: accounting reports and special reports. Special reports are required when "any unusual incident or circumstances lead the State to beljeve that there is or may have been Joss of nuclear material" that exceeds allowable limits, or when there has been an unexpected change in containment "to the extent that an unauthorized removal of nuclear material has become possible." Special reports may subsequently lead to special inspections, the purpose of which is to verify the reported information.

There are three kinds of accounting reports: initial reports, inventory change reports and material balance reports. The purpose of comparing records and reports is to ensure the consistency of the reported and recorded information.

The procedures followed are similar in nature to those of a records examination. The completeness, formal correctness and internal consistency of the reports must be established and the reports must be traced back to supporting evidence, namely, to the facility's records.

Recording of Book Inventory. In order to perform an inventory verification, the inspection team needs an up-to-date inventory listing. But, because of time lags in reporting, the information contained in reports is usually somewhat out-of-date. It is therefore necessary for the inspection tean to update the inventory listing on the basis of the operator's records. In facilities with computerized accounting systems, it is sometimes possible to obtain an up-to-date inventory listing without much difficulty. But in some cases, the task requires a significant effort.

Verification of Inventory Changes and/or Material Flow. In verifying inventory changes and material flow, IAEA inspectors count and identify items, make independent measurements and observe the operator's procedures. All items should be identified and counted and verification should be performed on a random sampling basis in accordance with the methodology described in Part $F$ of the Safeguards Technical Manual. (1) In a low-enriched uranium fabrication plant, flow verification poses a number of practical problems, the most important of which is the difficulty of meeting the requirements for strict randor sampling. The approach is to make periodic 
visits to the facility, at the rate of perhaps one visit every six weeks or every month, depending on the throughput and operating program.

Verification of Inventory. Verification of inventory is normally performed once or twice a year at low-enriched uranium fabrication plants, the actual frequency depending on the size of the facility. Verification activities include item counting and identification, independent measurements and observation of the operator's procedures. Verification of accounting data is on a random sampling basis, following the methodology described in Part $F$ of the Safeguards Technical Manual (appropriately modified to allow for practical constraints).

Verification of Quality and Functioning of Operator's Measurement System. To verify the quality and functioning of the operator's measurement system, Agency inspectors observe the operation and calibration of scales and other measuring equipment. The Agency is aiso provided with samples for destructive analysis. The results of these analyses can be used to monitor the operator's measurement errors.

Installation, Servicing or Verification of Containment and

Surveillance Devices. The scope of application of containment and surveillance is rather limited in low-enriched uranium fuel fabrication plants. Seals are used on containers of feed and product, and on static inventory items, i.e., items that are not expected to be used during a material balance period (stored scrap for example). Seals are used during inventory taking to guard against shuffing or substitution of items. Agency samples are sealed to protect against tampering.

Activities in Respect of MUF's, SRD's and Accidental Losses. Material unaccounted for (MUF), shipper/receiver differences (SRD's) and accidental losses all require careful investigation because of their potential use as a means to conceal diversion.

Post-Inspection and Evaluation Activities, Inciuding Trave1. Postinspection and evaluation activities include all those activities performed after the completion of the inspection; for example, travel (back to headquarters or to the next facility to be inspected), evaluation or processing of inspection data and the writing of reports. 
Inspection Plan

To assist in providing quantitative assessments of various aspects of the assumed inspection strategy, we make use of the INSPECT programs. (7) As input parameters for this example, we use:

- false alarmi probability $=\alpha=.055^{(a)}$

- goal non-detection probability $=\beta=.055(\mathrm{a})$

- goal quantity $=M=2,500 \mathrm{~kg}$ uranium at $3 \%$ enrichment $=75 \mathrm{~kg}$ uranium-235

- crossover point $=\gamma=.3$ (This quantity is related to the accuracy of the attributes test instruments.)

The facility data (material flows and measurement error standard deviations) are those described above. The inspector is assumed to verify the product from the plant in the form of fuel rods using the facility's rod scanner with independent calibration. The inspector's measurement error standard deviations for the various types of material are assumed the same as the operator's, except for fuel rods for which the following standard deviations apply:

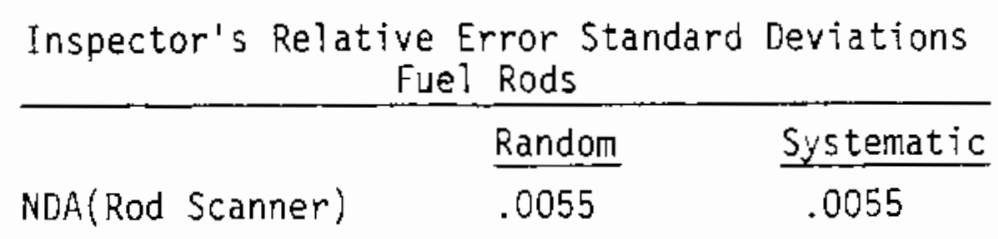

The first step is to create the five necessary data files. These include the following:

- The 400 ton plant stratum description file containing such things as the stratum name, average item weight, number of items, number of batches and measurement method indices.

- A bulk (weighing) measurement error file containing the random and systematic error standard deviations for each measurement method (scale) used by the operator or the inspector.

(a) The reasons for using this value are detailed in the Experimental Design and Data Generation section of this report (p. 52). 
- A file containing the inspector's and the operator's sampling error standard deviations.

- A file containing the inspector's and the operator's analytical error standard deviations.

- A file of between-laboratory error components (in this study, all these values are assumed to be zero).

Table 5 shows the bulk measurement error file, Table 6 the sampling error file, Table 7 the analytical error file, and Table 8 the between-laboratory components file. The stratum description file, which was given in Table 3, indicates the correspondence between strata and measurement method indices. (Recall that the notation in Table 3 was surmarized in Table 2.)

The next step is to convert these five data files into direct access files for later use. This is done using the programs SAMSIZ and RELVAR.

After conversion to direct access files, the next step is to calculate the variances of MUF and $\hat{D}$. This is done using the programs PROPER and DERR, respectively. DERR also assembles all the necessary inputs needed for calculating and evaluating sampling plans, and stores them in a direct access file. 
TABLE 5. Bulk Measurement Error File

BULK MEASUREMENT ERROR DATA

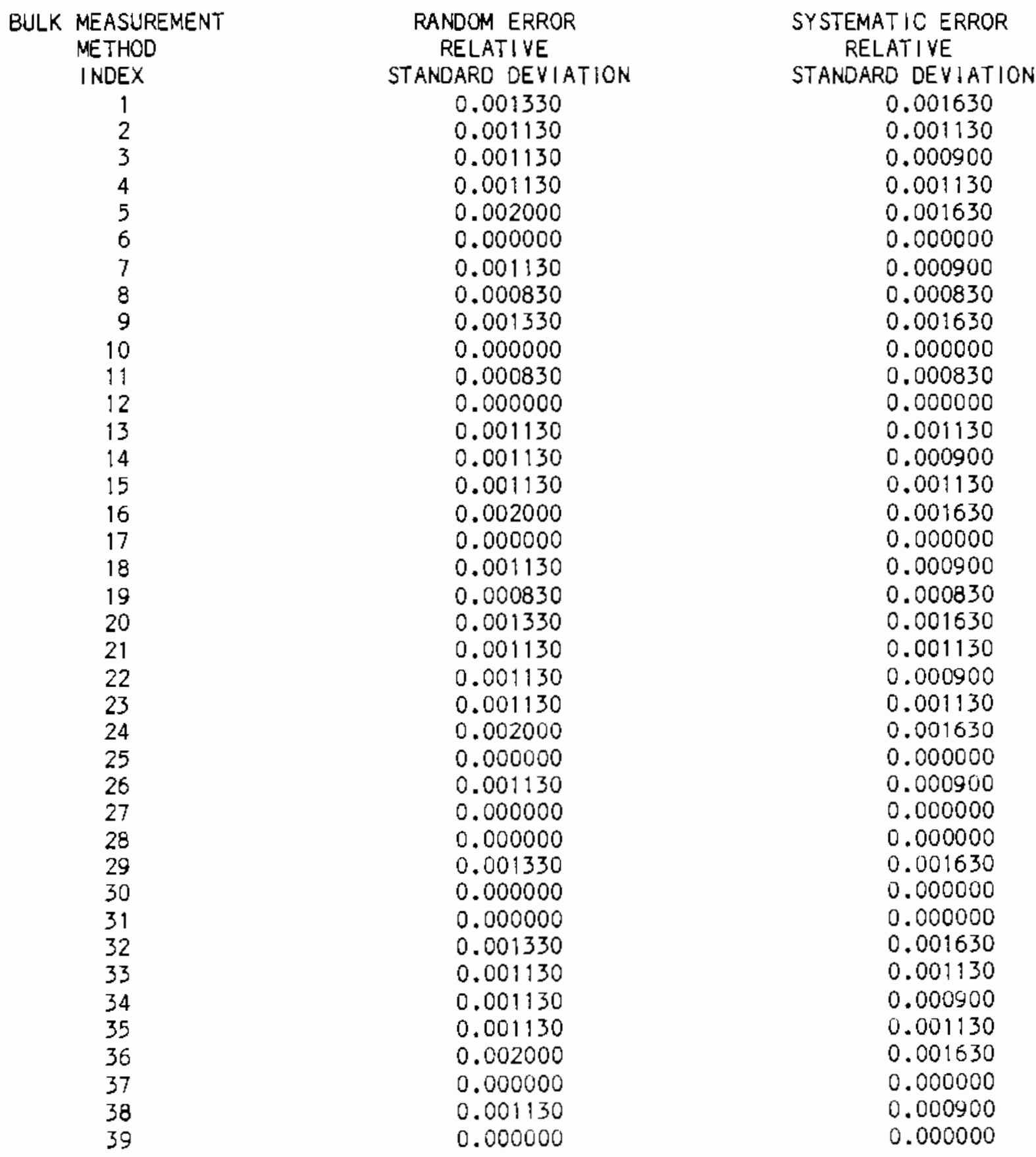


TABLE 6. Sampling Error File

SAMPLING
METHOD INDEX
1
2
3
4
5
6
7
8
9
10
11
12
13
14
15
16
17
18
19
20
21
22
23
24
25
26
27
28
29
30
31
32
33
34
35
36
37
38

SAMPL ING ERROR DATA

RANOOM ERROR

RELATIVE

STANDARD DEYIATION

0.001700

0.000000

0.000000

0.000000

0.000000

0.000000

0.000000

0.000000

0.001700

0.000000

0.000000

0.001700

0.000000

0.000000

0.000000

0.000000

0.000000

0.000000

0.000000

0.001700

0.000000

0.000000

0.000000

0.000000

0.000000

0.000000

0.000000

0.001700

0.000000

0.000000

0.001700

0.000000

0.000000

0.000000

0.000000

0.000000

0.000000

0.000000
SYSTEMATIC ERROR

RELATIVE

STANDARD DEVIATION

0.001440

0.000000

0.000000

0.000000

0.000000

0.000000

0.000000

0.000000

0.001440

0.000000

0.000000

0.001440

0.000000

0.000000

0.000000

0.000000

0.000000

0.000000

0.000000

0.001440

0.000000

0.000000

0.000000

0.000000

0.000000

0.000000

0.000000

0.001440

0.000000

0.000000

0.001440

0.000000

0.000000

0.000000

0.000000

0.000000

0.000000

0.000000 
TABLE 7. Analytical Error File

ANALYTICAL MEASUREMENT ERROR DATA

$\begin{array}{rc}\text { ANALYTICAL } & \text { RANDOM ERROR } \\ \text { RELATIVE } \\ \text { STANDARD DEVIATION } \\ 1 & 0.001480 \\ 2 & 0.001280 \\ 3 & 0.001200 \\ 4 & 0.002100 \\ 5 & 0.020000 \\ 6 & 0.087500 \\ 7 & 0.001820 \\ 8 & 0.001200 \\ 9 & 0.001480 \\ 10 & 0.001200 \\ 11 & 0.087500 \\ 12 & 0.001280 \\ 13 & 0.001200 \\ 14 & 0.002100 \\ 15 & 0.020000 \\ 15 & 0.087500 \\ 17 & 0.001820 \\ 18 & 0.001200 \\ 19 & 0.001480 \\ 20 & 0.001280 \\ 21 & 0.001200 \\ 22 & 0.002100 \\ 23 & 0.020000 \\ 24 & 0.087500 \\ 25 & 0.001820 \\ 26 & 0.005500 \\ 27 & 0.000000 \\ 28 & 0.001480 \\ 39 & 0.005500 \\ 31 & 0.087500 \\ 32 & 0.001480 \\ 33 & 0.001280 \\ 34 & 0.001200 \\ 36 & 0.002100 \\ 37 & 0.020000 \\ 38 & 0.087500 \\ & 0.001820 \\ 15 & 0.005500 \\ 13 & \end{array}$

SYSTEMATIC ERROR
RELATIVE
STANDARD DEVIATION
0.001400
0.001200
0.000980
0.002100
0.016300
0.056250
0.001630
0.001200
0.001400
0.001200
0.056250
0.001200
0.000980
0.002100
0.016300
0.056250
0.001630
0.001200
0.001400
0.001200
0.000980
0.002100
0.016300
0.056250
0.001630
0.005500
0.000000
0.001400
0.005500
0.056250
0.001400
0.001200
0.000980
0.002100
0.016300
0.056250
0.001630
0.005500


TABLE 8. Between-Laboratory Components File

BETWEEN-LABORATORIES ERROR COMPONENTS

$\begin{array}{cc}\text { BETWEEN LABORATORY } & \text { BETWEEN-LABORATORIES } \\ \text { COMPONENT INDEX } & \text { ERROR COMPONENT } \\ 1 & 0.000000 \\ 2 & 0.000000 \\ 3 & 0.000000 \\ 4 & 0.000000 \\ 5 & 0.000000 \\ 6 & 0.000000 \\ 7 & 0.000000 \\ 8 & 0.000000 \\ 9 & 0.000000 \\ 10 & 0.000000 \\ 11 & 0.000000 \\ 12 & 0.000000 \\ 13 & 0.000000 \\ 14 & 0.000000 \\ 15 & 0.000000 \\ 16 & 0.000000 \\ 17 & 0.000000 \\ 18 & 0.000000 \\ 19 & 0.000000 \\ 20 & 0.000000 \\ 21 & 0.000000 \\ 22 & 0.000000 \\ 23 & 0.000000 \\ 24 & 0.000000 \\ 25 & 0.000000 \\ 26 & 0.000000 \\ 27 & 0.000000 \\ 28 & 0.000000 \\ 29 & 0.000000 \\ 30 & 0.000000 \\ 31 & 0.000000 \\ 33 & 0.000000 \\ 34 & 0.000000 \\ 35 & 0.000000 \\ 36 & 0.000000 \\ 37 & 0.000000 \\ 38 & 0.000000 \\ 39 & 0.000000 \\ & 0.000000 \\ 32 & \\ 38 & \end{array}$


Table 9 contains the output from PROPER, that is, it contains the variance components of MUF. Table 10 contains the output from DERR, namely, the variance components of the $\hat{0}$ statistic.

The final step is to run the program INSPCF to calculate and evaluate the sampling plans. For this example the resulting outputs are shown in Tables 11, 12 and 13. A description of these results follows.

The attributes sampling plan (Table 11) with two inventories calls for a total of 934 samples. The largest components are the fuel rod stratum in removais (459) and the $U F_{6}$ cylinder stratum in additions to plant (225). The large fuel rod sample size primarily reflects the fact that the straturi is large $(136,710$ items $)$. The large number of $U F_{6}$ samples reflects the fact that each cylinder contains a large fraction of the goal quantity. These samples would be taken over a one year period in probably eight or nine flow verification inspections. The inventory sample sizes are not large reflecting the relatively small size of inventory compared to throughput.

Table 12 shows the variables sampling plan for attributes mode $\left(n_{v 1}\right)$, and the variables sampling plan for detection of bias defects $\left(n_{v 2}\right)$. These sample sizes are all smaller than the attributes sampling plan already discussed. 
TABLE 9. Variance of MUF

\section{CASE 29 MUF CALCULATION}

VARIANCE OF MUF -- RANDOM ERROR COMPONENTS

STRATUM

WEIGHT VARIANCE DUE TO
WEIGHING

BEGINNING INYENTORY

$\begin{array}{lrr}\text { UF6 CYL INDERS } & 58000.000 & 148.7645 \\ \text { U02 POWDER } & 25740.000 & 0.4273 \\ \text { U02 PELLETS } & 13260.000 & 0.2201 \\ \text { U308 POWDER } & \mathbf{4 0 0 0 . 0 0 0} & 0.1022 \\ \text { SCRAP } & 20000.000 & 0.8000 \\ \text { WASTE } & 500.000 & 0.0000 \\ \text { MISCELLANEOUS } & 2000.000 & 0.0102 \\ \text { FUEL RODS } & 11692.801 & 0.0234\end{array}$

ADDITIONS TO PLANT

$\omega$
400200.000

REMOVALS FROM PLANT

10 RODS

11 WASTE

396459.000

3741.000

ENDING INVENTORY

$\begin{array}{lr}\text { UF6 CYLINDERS } & 58000.000 \\ \text { U02 POHDER } & 25740.000 \\ \text { U02 PELLETS } & 13260.000 \\ \text { U308 POWDER } & 4000.000 \\ \text { SCRAP } & 20000.000 \\ \text { WASTE } & 500.000 \\ \text { MISCELLANEOUS } & 2000.000 \\ \text { FUEL RODS } & 11692.801\end{array}$

TOTALS
1026.4750

81.0163

0.0000

0.0000

0.0000

0.0000

0.0000

0.0000

0.0000

559.0127

0.0000

0.7920

0.0000

0.0000

81.0163

0.0000

0.0000

0.0000

0.0000

0.0000

0.0000

0.0000

0.0102

0.0234

0.0000

721.0454

1327.9623
VARIANCE DUE TO ANALYSIS

VAFI ANCE TOTAL

$$
\begin{array}{r}
291.1851 \\
8.6509 \\
2.7024 \\
3.6302 \\
2000.8000 \\
1.9141 \\
0.0235 \\
1.0488
\end{array}
$$

0.0132

1.0254

423.6891

2009.1768

34.7679

14.3210

35.5599

14.3210

$$
\begin{array}{r}
61.4042 \\
8.2236 \\
2.4823 \\
3.5280 \\
2000.0000 \\
1.9141 \\
0.0132 \\
1.0254
\end{array}
$$

291.1851
8.6509
2.7024
3.6502

3.6302
1.8000

1.9141

0.0235

1.0488 
TABLE 9. (continued)

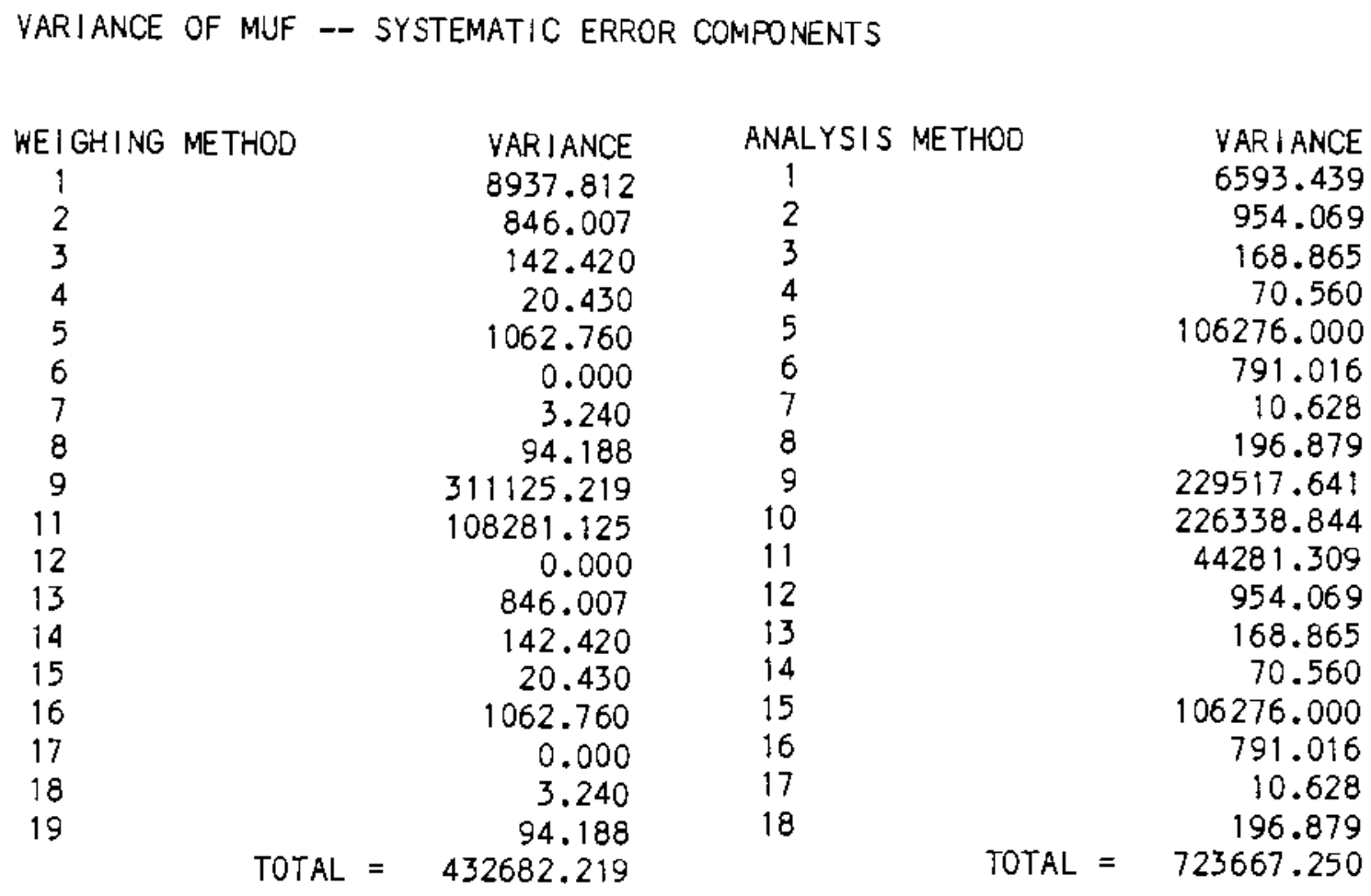

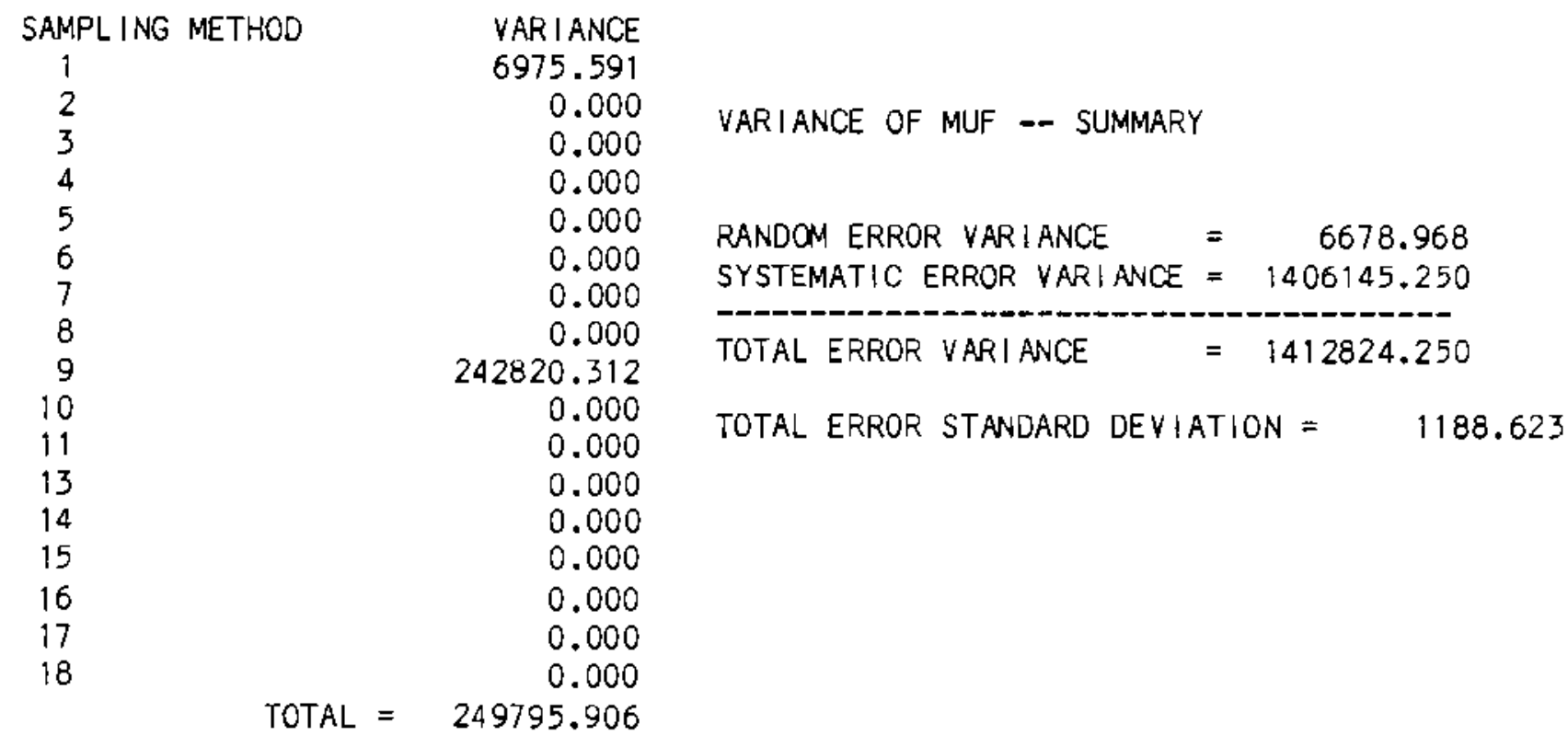

TOTAL SYSTEMATIC ERROR VAFI ANCE OF MUF $=1406145.250$ 
TABLE 10. Variance of $\hat{D}$

\begin{tabular}{|c|c|c|}
\hline WEIGHING METHOD & $\begin{array}{l}\text { OPERATOR'S } \\
\text { CONTRIBUTION }\end{array}$ & $\begin{array}{l}\text { INSPECTOR'S } \\
\text { CONTRI BUTION }\end{array}$ \\
\hline 1 & 8937.8115 & 0.0000 \\
\hline 2 & 846.0070 & 0.0000 \\
\hline 3 & 142.4203 & 0.0000 \\
\hline 4 & 20.4304 & 0.0000 \\
\hline 5 & 1062.7599 & 0.0000 \\
\hline 6 & 0.0000 & 0.0000 \\
\hline 7 & 3.2400 & 0.0000 \\
\hline 8 & 94.1875 & 0.0000 \\
\hline 9 & 311125.2187 & 0.0000 \\
\hline 10 & 0.0000 & 0.0000 \\
\hline 11 & 108281.1250 & 0.0000 \\
\hline 12 & 0.0000 & 0.0000 \\
\hline 13 & 846.0070 & 0.0000 \\
\hline 14 & 142.4203 & 0.0000 \\
\hline 15 & 20.4304 & 0.0000 \\
\hline 16 & 1062.7599 & 0.0000 \\
\hline 17 & 0.0000 & 0.0000 \\
\hline 18 & 3.2400 & 0.0000 \\
\hline 19 & 94.1875 & 0.0000 \\
\hline 20 & 0.0000 & 8937.8115 \\
\hline 21 & 0.0000 & 846.0070 \\
\hline 22 & 0.0000 & 142.4203 \\
\hline 23 & 0.0000 & 20.4304 \\
\hline 24 & 0.0000 & 1062.7599 \\
\hline 25 & 0.0000 & 0.0000 \\
\hline 26 & 0.0000 & 3.2400 \\
\hline 27 & 0.0000 & 0.0000 \\
\hline 28 & 0.0000 & 0.0000 \\
\hline 29 & 0.0000 & 425529.1875 \\
\hline 30 & 0.0000 & 0.0000 \\
\hline 31 & 0.0000 & 0.0000 \\
\hline 32 & 0.0000 & 8937.8115 \\
\hline 33 & 0.0000 & 846.0070 \\
\hline 34 & 0.0000 & 142.4203 \\
\hline 35 & 0.0000 & 20.4304 \\
\hline 36 & 0.0000 & 1062.7599 \\
\hline 37 & 0.0000 & 0.0000 \\
\hline 38 & 0.0000 & 3.2400 \\
\hline \multirow[t]{2}{*}{39} & 0.0000 & 0.0000 \\
\hline & 432682.2187 & 447554.5000 \\
\hline
\end{tabular}


TABLE 10. (continued)

\begin{tabular}{|c|c|c|}
\hline SAMPL ING METHOD & $\begin{array}{l}\text { OPERATOR'S } \\
\text { CONTRIBUT!ON }\end{array}$ & $\begin{array}{l}\text { INSPECTOR'S } \\
\text { CONTRIBUTION }\end{array}$ \\
\hline 1 & 6975.5913 & 0.0000 \\
\hline 2 & 0.0000 & 0.0000 \\
\hline 3 & 0.0000 & 0.0000 \\
\hline 4 & 0.0000 & 0.0000 \\
\hline 5 & 0.0000 & 0.0000 \\
\hline 6 & 0.0000 & 0.0000 \\
\hline 7 & 0.0000 & 0.0000 \\
\hline 8 & 0.0000 & 0.0000 \\
\hline 9 & 242820.3125 & 0.0000 \\
\hline 10 & 0.0000 & 0.0000 \\
\hline 11 & 0.0000 & 0.0000 \\
\hline 12 & 0.0000 & 0.0000 \\
\hline 13 & 0.0000 & 0.0000 \\
\hline 14 & 0.0000 & 0.0000 \\
\hline 15 & 0.0000 & 0.0000 \\
\hline 16 & 0.0000 & 0.0000 \\
\hline 17 & 0.0000 & 0.0000 \\
\hline 18 & 0.0000 & 0.0000 \\
\hline 19 & 0.0000 & 0.0000 \\
\hline 20 & 0.0000 & 6975.5913 \\
\hline 21 & 0.0000 & 0.0000 \\
\hline 22 & 0.0000 & 0.0000 \\
\hline 23 & 0.0000 & 0.0000 \\
\hline 24 & 0.0000 & 0.0000 \\
\hline 25 & 0.0000 & 0.0000 \\
\hline 26 & 0.0000 & 0.0000 \\
\hline 27 & 0.0000 & 0.0000 \\
\hline 28 & 0.0000 & 332107.8750 \\
\hline 29 & 0.0000 & 0.0000 \\
\hline 30 & 0.0000 & 0.0000 \\
\hline 31 & 0.0000 & 6975.5913 \\
\hline 32 & 0.0000 & 0.0000 \\
\hline 33 & 0.0000 & 0.0000 \\
\hline 34 & 0.0000 & 0.0000 \\
\hline 35 & 0.0000 & 0.0000 \\
\hline 36 & 0.0000 & 0.0000 \\
\hline 37 & 0.0000 & 0.0000 \\
\hline 38 & 0.0000 & 0.0000 \\
\hline & 249795.9062 & 346059.0625 \\
\hline
\end{tabular}


TABLE 10. (continued)

$\begin{array}{lrr}\text { ANALYSIS METHOD } & \text { OPERATOR'S } & \text { INSPECTOR'S } \\ & \text { CONTRIBUTION } & \text { CONTRIBUTION } \\ 1 & 6593.4395 & 0.0000 \\ 2 & 954.0687 & 0.0000 \\ 3 & 168.8648 & 0.0000 \\ 4 & 70.5600 & 0.0000 \\ 5 & 106276.0000 & 0.0000 \\ 6 & 791.0156 & 0.0000 \\ 7 & 10.6276 & 0.0000 \\ 8 & 196.8791 & 0.0000 \\ 9 & 229517.6406 & 0.0000 \\ 10 & 226338.8437 & 0.0000 \\ 11 & 44281.3086 & 0.0000 \\ 12 & 954.0687 & 0.0000 \\ 13 & 168.8648 & 0.0000 \\ 14 & 70.5600 & 0.0000 \\ 15 & 106276.0000 & 0.0000 \\ 16 & 791.0156 & 0.0000 \\ 17 & 10.6276 & 0.0000 \\ 18 & 196.8791 & 0.0000 \\ 19 & 0.0000 & 6593.4395 \\ 20 & 0.0000 & 954.0687 \\ 21 & 0.0000 & 168.8648 \\ 22 & 0.0000 & 70.5600 \\ 23 & 0.0000 & 106276.0000 \\ 24 & 0.0000 & 791.0156 \\ 25 & 0.0000 & 10.6276 \\ 26 & 0.0000 & 4135.8276 \\ 27 & 0.0000 & 0.0000 \\ 28 & 0.0000 & 313913.6562 \\ 29 & 0.0000 & 4754686.5000 \\ 30 & 0.0000 & 44281.3086 \\ 31 & 0.0000 & 6593.4395 \\ 32 & 0.0000 & 954.0687 \\ 33 & 0.0000 & 168.8648 \\ 34 & 0.0000 & 70.5600 \\ 35 & 0.0000 & 106276.0000 \\ 36 & 0.0000 & 791.0156 \\ 37 & 0.0000 & 10.6276 \\ 38 & 0.0000 & 4135.8276 \\ & & \\ & 723667.2500 & 5350882.5000\end{array}$


TABLE 10. (continued)

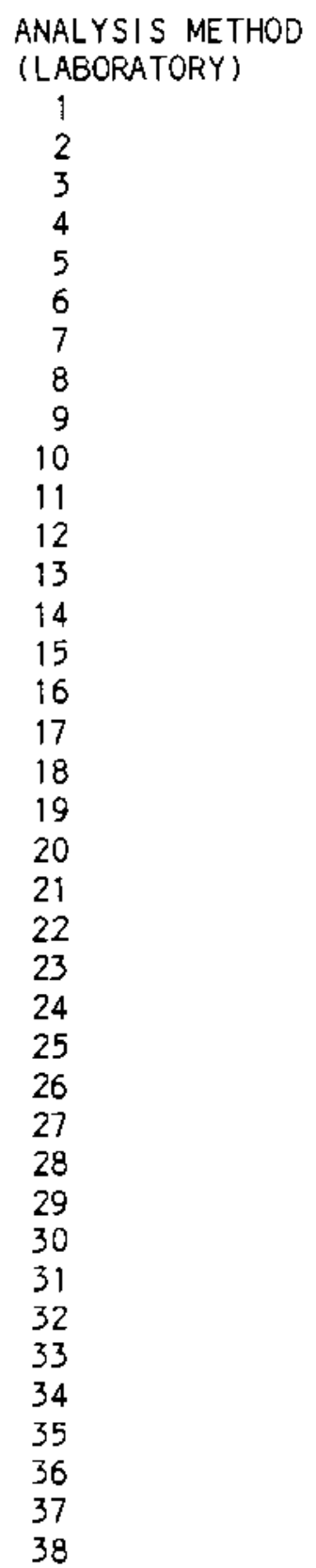

\section{OPERATOR'S}

CONTRIBUT ION

0.0000

0.0000

0.0000

0.0000

0.0000

0.0000

0.0000

0.0000

0.0000

0.0000

0.0000

0.0000

0.0000

0.0000

0.0000

0.0000

0.0000

0.0000

0.0000

0.0000

0.0000

0.0000

0.0000

0.0000

0.0000

0.0000

0.0000

0.0000

0.0000

0.0000

0.0000

0.0000

0.0000

0.0000

0.0000

0.0000

0.0000

0.0000
I NSPECTOR'S

CONTRI BUT ION

0.0000

0.0000

0.0000

0.0000

0.0000

0.0000

0.0000

0.0000

0.0000

0.0000

0.0000

0.0000

0.0000

0.0000

0.0000

0.0000

0.0000

0.0000

0.0000

0.0000

0.0000

0.0000

0.0000

0.0000

0.0000

0.0000

0.0000

0.0000

0.0000

0.0000

0.0000

0.0000

0.0000

0.0000

0.0000

0.0000

0.0000

0.0000

VARIANCE OF D -- SUMMARY

RANDOM ERROR YARIANCE $=0.000$

SYSTEMATIC ERROR YARI ANCE $=7550641.500$

TOTAL ERROR YARIANCE $=7550641.500$

TOTAL ERROR STANDARD DEVIATION $=2747.843$ 
IABLE 11. Attributes Sampling Plan

CASE 29

SAMPLE SIZES FOR THE ATTRIBUTES TEST

THE NON-DETECTION PROBABILITY FOR LARGE DEFECTS IS SET AT 0.055 STRATUM

STRATUM DESCRIPTION

WE I GHTT NUNMER

BEGINNING INVENTORY

$\begin{array}{ll}1 & \text { UF6 CYLINDERS } \\ 2 & \text { UO2 POWDER } \\ 3 & \text { UO2 PELLETS } \\ 4 & \text { U3O8 POWDER } \\ 5 & \text { SCRAP } \\ 6 & \text { WASTE } \\ 7 & \text { MISCELLANEOUS } \\ 8 & \text { FUEL RODS }\end{array}$

1450.000

$13.000-1980$

$13.000-1020$

$20.000 \quad 200$

$10.000 \quad 2000$

$0.500 \quad 1000$

$4.000 \quad 500$

$2.900 \quad 4032$

ADDITIONS TO PLANT

$1 \quad$ UF 6 CYLINDERS

REMOVALS FROM PLANT

$2.900 \quad 136710$

$0.500 \quad 7482$

ENDING INVENTORY

UF 6 CYL INDERS
UO2 POWDER
U02 PELLETS
U3O8 POWDER
SCRAP
WASTE
MI SCELLANEQS
FUEL RODS

1450.000

13.000

13.000

20.000

10.000

0.500

4.000

2.900

TOTALS

166012
GOAL QUANTITIES 2500.0

33

30

15

5

23

1

2

14

225

459

4

33

30

15

5

23

1

2
14

934 


\section{TABLE 12. Variables Sampling Plan}

LOWER BOUND FOR YAR DIFANOCM $3 / \mathrm{HO}=$

POINT OF DIMINISHING HE IURNS=

5351.0

VAN + ANCE INFLATION + ACTORE 4.000

LOWER GOUND FOR VAK DIRANDOM

UNOER HO USING DIMINISHING RETUKNS CONCEHT=

UHPER BOUND FOR VAR D(KANOOM $) / H O=$

$0.7384 \mathrm{EE}+07$
LOWER GOUAO AS SAWLE SIZE APFROACHES INFINI T

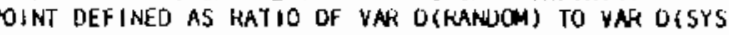

BEYOND WHICH TAKING MORE SAHULES IS NOT OOST EFFECTIVE

FATIO OF VAR D(KANDOM)/HI YO VAR O (KANDOM)/HO

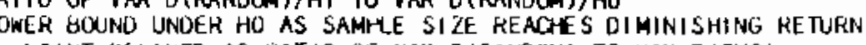
POINT DEFINED AS RATIO OF VAR DIRANDOM) TO VAR DISYS) UHFH BOUN ASSUMING AT LEAST ONE SAMLLE HER STRATUM

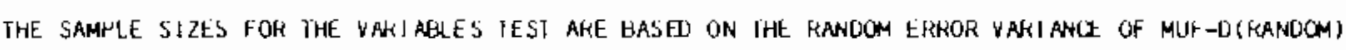

NOIATION:

NY = SAMIJLE SIZE FOR PAFTIIAL DEFELTS NOT DETECIAGLE GY THE ATTRIGUTES TEST

WWL = SAMPLE SIZE FOR BIAS UEFECIS

$N V=\operatorname{MAX}(N Y), N Y 2)$

G- THE INOUCED PKOBABILITYY OH NON-DETELIIION BASEO ON NYZ

- CRUSSDVER FACTOR

i.- RAIIO OF VAR OLRANDOMI/HI TO VRH DESYSTEMATIC)

STRATUM

$$
\begin{aligned}
& 2500.0 \\
& 0.803= \\
& u .250=L \\
& \text { C NVI NY2 NV }
\end{aligned}
$$

$\begin{array}{lll}\text { BEGINNING INYENTORY } \\ 30 & 16 & 2\end{array}$
GOAL QUANTIIY $\begin{array}{rrrr}.30 & 16 & 2 & 16 \\ .30 & 9 & 1 & 9\end{array}$

.30

.30

.30

.50 loy IS

109

FEMOVRLS FHOM PLANI

$\begin{array}{ccc}.30 & 130 & 27\end{array}$

ENDINGS INYENTOKY

$\begin{array}{cccc}.30 & 16 & 2 & 16 \\ .30 & 9 & 1 & 9 \\ .30 & 5 & 0 & 5 \\ .30 & 1 & 0 & 1 \\ .30 & 7 & 6 & 7 \\ .30 & 0 & 1 & 1 \\ .30 & 1 & 0 & 1 \\ .30 & 4 & 1 & 4\end{array}$

FUEL RDOS

NW1 TO1A

NV2 TOTAL

TOTAL 


\section{TABLE 13. Effectiveness of Sampling Plans}

CASE 29

THE FALSE ALAKM PKOLAEUILITY IS SET AT 0.026

THE NOH-DETECTION HKOHABILITY HOR LARGE DEFECTS IS SET AT 0.055

SUMMARY OF CALCLAATIONS FOH THE DE TELTION OF RARTIA OEFECTS ANO BIAS

GOAL
QUANTITY

\begin{tabular}{|c|c|c|c|}
\hline NAT TAKE & $\begin{array}{l}\text { NON- LE TEQT ION } \\
\text { HHOUAB! I.ITY }\end{array}$ & $\begin{array}{l}\text { WON-DE TECI ION } \\
\text { HROBABIL ITY }\end{array}$ & $\begin{array}{c}\text { TOIAL } \\
\text { NON-BETECT ION }\end{array}$ \\
\hline AKGE DEFEC & TOK & $\begin{array}{l}\text { TOR } \\
\text { MUH }\end{array}$ & FRGSABILITY \\
\hline
\end{tabular}

ROBABILITK

OF

DETECTION

TOTAL
MEASUREMENTS
IO DETECT
GIAS

LASUREMENTS

TO DETECT

IAS ANB

TO DETECTS $\mathrm{A}$

GIAS FARTIAL DEFECTS

TO DETECT

DEFECTS

$\begin{array}{llllll}2500.0 & 0.0 & 1.000 & 0.821 & 0.821 & 17.9 \\ 2500.0 & 0.0 & 1.000 & 0.811 & 0.011 & 18.9\end{array}$

17.9

71

341

$934 \quad 1.0$
4.0

YAR MUF $=0.14061 E+0 \%$

VAR U(SYSTEMATIC) $=0.75500 E+07$

GOAL

QUANTIIY VAK OIKRNOOM)/HO

2500.00

131685.672 $\checkmark A R \mathrm{D} / \mathrm{HO}$

VAK O(RANOOM)/HI
G86742.687

VAR $0 / H 1$
8237384.000

VRR MUF $-D / H O$

6316182.000

VAK $\mathrm{MUT}-\mathrm{O} / \mathrm{H} / \mathrm{H}$ 
The number of variables samples in the attributes mode $\left(n_{v i}\right)$ is smaller because a larger number of defects is required to divert a goal quantity via partial defects, hence this path is easier to detect. The sample sizes to detect bias are moderate. Since the actual variables sample size must be adequate to detect both bias and partial defects, the larger of $n_{v}$ and $n_{v 2}$ in each stratum is chosen as the actual variables sample size. For case 29 of the experimental design, this is shown to be 341 .

Once the sampling pian has been determined, the computer code evaluates the effectiveness using the QMax procedure. (See Table 13.) for case 29 of the experimental design, the probability of detection is only about $18 \%$.

An interesting conclusion can be drawn from this example. First, note that the standard deviation of MUF is $1186 \mathrm{~kg}$ over a one year period. The throughput over the same period is $400,000 \mathrm{~kg}$. Thus, the relative standard deviation of MUF is $\sim 0.3 \%$, which is exactly the same as the international standard of accountability in uranium fabrication facilities. Even with measurements comparable to the international standard of accountability, the probability of detection was only $18 \%$. The international standard by itself does not ensure a high probability of detecting the diversion of $75 \mathrm{~kg} U$. Many other factors must be considered as well.

Estimation of Inspection Effort

To simplify the estimation procedure, the inspection tasks outlined earlier are compressed into five major cateogries as follows:

- Planning and Preparation (including follow up actions from previous inspections)

- Audit (inciudes records examination, comparison between records and reports, recording of book inventory, verification of quality and functioning of operator's measurement system and activities in respect to MUF's, SRO's, and accidental losses)

- Check and service containment and surveillance devices

- Post-inspection and evaluation

- Verification of measurement data (includes verification of inventory changes and/or material flow and verification of inventory) 
Table 14 summarizes the inspection effort required for routine inspections in a one year period at the example facility. Appendix A explains how the estimates of inspection effort were arrived at in this study.

TABLE 14. Total Inspection Effort for Routine Inspections (One-Year Period)

\begin{tabular}{|c|c|c|c|c|c|}
\hline & $\begin{array}{l}\text { Man-days } \\
\text { at Facility }\end{array}$ & $\begin{array}{l}\text { Man-days } \\
\text { Travel } \\
\end{array}$ & $\begin{array}{l}\text { Man-days } \\
\text { in Field } \\
\end{array}$ & $\begin{array}{l}\text { Man-days } \\
\text { at } \mathrm{HQ}\end{array}$ & $\begin{array}{l}\text { Total } \\
\text { Man-days } \\
\end{array}$ \\
\hline $\begin{array}{l}\text { Planning and } \\
\text { Preparation }\end{array}$ & 0 & 28 & 28 & 34 & $6 ?$ \\
\hline Audit & 43 & 0 & 43 & 0 & 43 \\
\hline $\begin{array}{l}\text { Verification of } \\
\text { Measurement } \\
\text { Data }\end{array}$ & 82 & 0 & 82 & 0 & 82 \\
\hline $\begin{array}{c}\text { Check and Service } \\
\text { C/S Devices }\end{array}$ & 1 & 0 & 1 & 0 & 1 \\
\hline $\begin{array}{l}\text { Post-Inspection } \\
\text { and Evaluation }\end{array}$ & 0 & 28 & 28 & 36 & 64 \\
\hline Tota & 126 & 56 & 182 & 70 & 252 \\
\hline
\end{tabular}


INPUTS TO THE ANALYSIS

There are three classes of inputs to the method of C.5: those pertaining to the facility model, those pertaining to the safeguards goa?s, and those pertaining to the safeguards approach.

Inputs Pertaining to the Facility Model

As explained in ISP0-58, PNL-2559, ${ }^{(7)}$ the necessary facility information consists of stratum description information and measurement error information. See Figure 4.

\section{Stratum Description Information}

The stratum description information includes a complete, stratified material balance. For each stratum, the following aspects of the operator's material accounting systen must be specified:

- stratum name

- average item weight

- the bulk measurement method used

- the material sampling method used

- the anarytical method used

- the number of batches

- the (average) number of items per batch

- the number of samples per batch

- the number of analyses per sample

There are also a number of other aspects of the plant, aggregated from the basic stratum information, that can be considered as inputs to be varied. Some obvious examples are the throughput and the inventory, al though there are many other possibilities such as the total number of strata, the ratio of throughput to inventory, the total number of chemical analyses performed in a materiai balance period.

Measurement Error Information

For each weighing, sampling and analytical measurement method used in the facility, both random and systematic error standard deviations must be specified. 


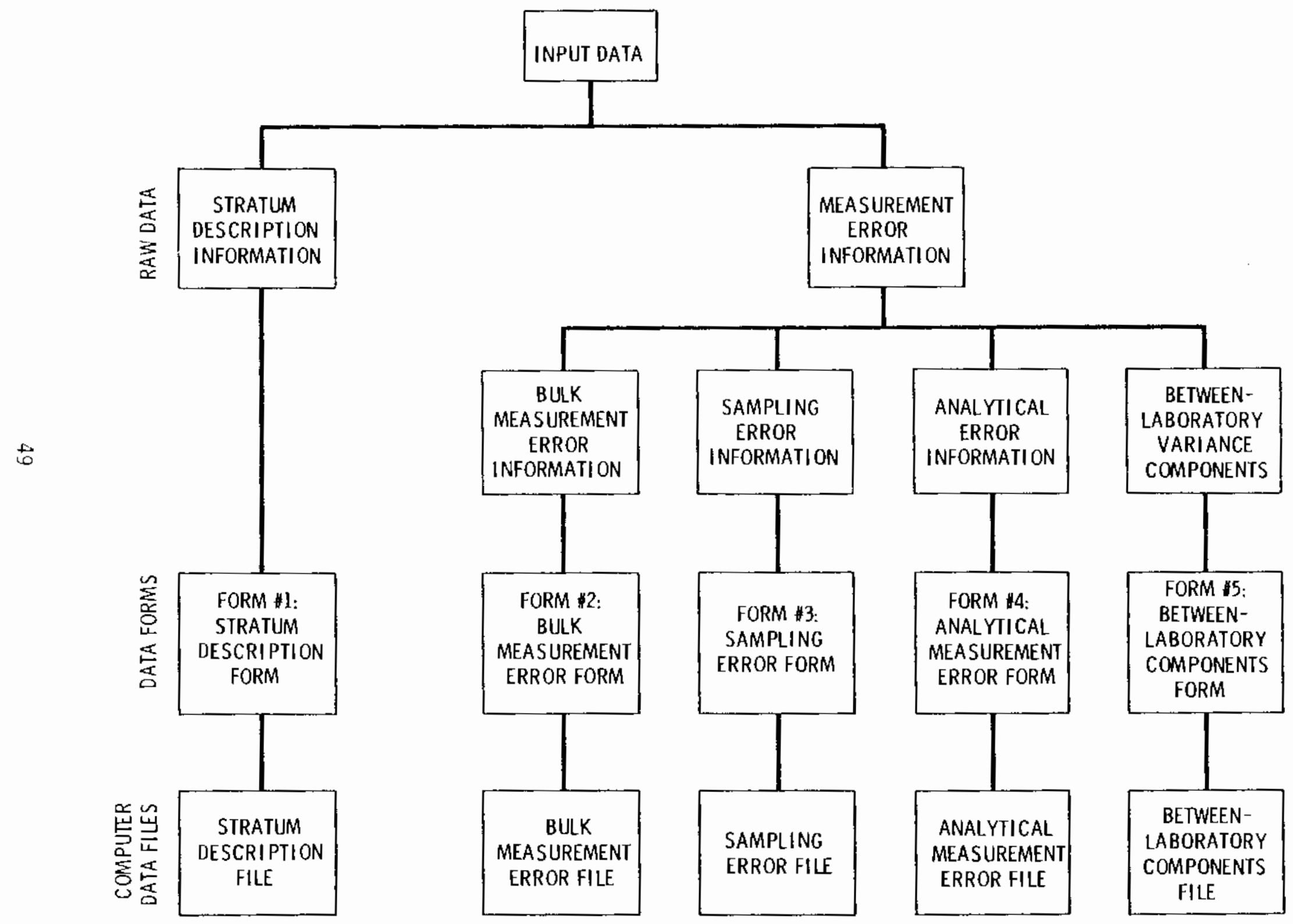

FIGURE 4. Input Data 
On a more macroscopic level, one could consider the standard deviation of MUF as a percent of throughput to be a factor that one could vary. This ratio is related to the so-called international standard of accountability and one might wish to explore, say, the effects on the safeguards system of improvements in the international standard. However, in practice, it would be necessary to work the problem on the level of the more fundamental measurement error information involving individual measurement methods. It is more instructive to focus on those particular measurement methods that contribute strongly to the variance of MUF, rather than to focus on the variance of MUF itself as a parameter to be varied.

Inputs Pertaining to the Safeguards Goals

Among the parameters that are related to the technical objectives of safeguards, the following are the most important:

- goal quantity, GQ

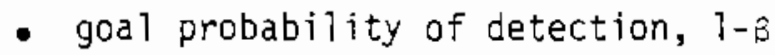

- goal false alarm probability, a

- detection time

- variance lower bound factor (7)

- variance inflation factor(7)

Inputs Pertaining to the Safeguards Approach

There are several alternative safeguards approaches that can be considered for use in LEU conversion-fabrication plants. The ma in differences between them involve the inspector's method of verifying the product from the plant. Three basic possibilities are:

1. Verify product in the form of finished fuel assemblies prior to shipment

2. Verify product in the form of fuel rods prior to assembly

3. Verify product in the form of pellet stacks prior to loading into fuel rods 
For any particular inspection approach, the following inputs must be specified:

- The inspector's measurement strategy must be defined.

- Measurement error standard deviations must be specified for each kind of verification measurement. This includes the definition of the crossover point for each attributes test technique.

- The number of analyses per item selected for destructive verification measurement.

- The number of samples per batch for which the inspector makes a destructive element determination.

SELECTION OF FACTORS OF INTEREST

Although one might consider studying the effects of changing any one of the input factors, not all of the factors are of equal importance or interest. The purpose of Task $C .19$ is to analyze the impact of safeguards criteria. For this reason, we have chosen to concentrate on those input factors related to safeguards goals. However, we have also included two factors involving the facility model--namely the annual plant throughput and the operator's measurement capability--and two factors involving the safeguards approach--namely the inspector's variables measurement capability and the inspector's attributes test crossover point. It is felt that these factors play a sufficientiy important role in determining the efficiency and effectiveness of safeguards that they merit more detailed analysis.

The factors retained for detailed analysis are listed in Table 15. 
TABLE 15. Factors Selected for Detailed Analysis

False alarm probability

Goal probability of non-detection

Goal quantity

Operator's measurement capability

Inspector's variables measurement capability

Inspector's attribute measurement capability

Plant size (annual throughput) 
IDENTIFICATION ANO SELECTION OF RESPONSES OF INTEREST

The list of possibie responses that could be considered is very long indeed. It is convenient to divide them into two groups: those related to the costs or efficiency of safeguards and those related to the effectiveness of safeguards.

Responses Related to Effectiveness

The key response here is obviously the probability of detection. The INSPECT programs calculate a parameter called $Q_{\text {MAX }}$, which is the probability of non-detection based on the diverter's optimal diversion strategy. The probability of detection is $1-Q_{\text {MAX }}$.

Other parameters reiated to effectiveness include:

- the variance and standard deviation of MUF

- the variance and standard deviation of $\hat{D}$

- the variance and standard deviation of MUF- $\hat{D}$

- various components of the variances of MUF, $\hat{D}$, and MUF- $\hat{D}$

Many other responses could be cited. The ones mentioned here are felt to include the most significant ones. Although $0_{\text {MAX }}$ is the response of major interest, some attention is given to the others as well.

Responses Related to Efficiency

The responses of interest involve various aspects of the costs incurred by the IAEA in implementing safeguards. Note that we do not consider in this study costs incurred by facilities and states as a result of IAEA safeguards.

The responses of most interest are total man-days of inspection effort and man-days of inspection effort at facilities (which is related to ARIE and MRIE). Other responses considered include:

- attributes sample sizes

- variables sample sizes

- inspection effort required for sampling

- inspection effort required for planning and preparing for inspections 
- inspection effort required for auditing

- inspection effort required for post-inspection and evaluation activities

EXPERIMENTAL DESIGN AND DATA GENERATION

Seven input factors have been identified:

- faise alarm probability, a

- non-detection probability, $\beta$

- detection goal quantity, GQ

- operator's measurement capability

- inspector's measurement capabijity

- inspector's attributes test crossover point, $r$

- plant size (annual throughput)

It is desired to explore the effect each of these factors has on:

a) Q $Q_{\text {MAX }}$ (the probability of non-detection assuming the diverter's optimal strategy) and inspection effort, and b) a variety of other responses. The kind of information we would like to obtain is:

- whether the effect is positive or negative

- the magnitude of the effect

- whether the effect is linear in the factor or non-linear

- whether there are interactions between factors

\section{Region of Interest}

In order to focus our experimental effort on areas of most interest, we specify for each input factor a range of values that will be considered in the analysis.

False Alarm Probability

Various working groups have recommended that false alarm probabilities should be in the range of $1 \%$ to $10 \%$. We interpret this to refer to the probability per facility per year of deciding to initiate follow-up actions, i.e., to trigger an investigation. 


\section{Non-detection Probability}

Various working groups have recommended detection probabilities in the range of $90 \%$ to $99 \%$. Again, we interpret detection to mean the decision to infiate an investigation.

Goal Quantity

For uranium of low-fissile content, the quantity of safeguards significance has been defined as 75 kilograms of uranium-235 contained in lowenriched, natural, or depleted uranium. We have arbitrarily defined the range of goal quantities to be considered in our sensitivity study as $25 \mathrm{~kg}$ to $125 \mathrm{~kg}$ of uranium-235, in other words $75 \pm 50 \mathrm{kilograms}$.

Operator's Measurement Capability

After an extensive examination of the literature on measurement methods, we selected ranges of values for random and systematic error standard deviations. Tabie 16 shows the ranges chosen for each type of material. The low values are quite optimistic. The very best facilities currently in operation may come close to these values but most facilities will not. The high values are quite pessimistic; most current facilities probabiy do better. The medium or average values are consistent with the international standard of accountability at LEU conversion-fabrication plants; again, many facilities can do somewhat better.

Inspector's Measurement Capability

Where the inspector uses destructive analysis methods for making verification measurements, we have assumed the same range of measurement error standard deviations as for the operator.

Where the inspector uses non-destructive measurements for flow verification, we have again examined the literature and established a range of values that is believed to bracket the values likely to apply in practice.

Table 17 shows the assumptions we have made about the inspector's measurement error standard deviations. 
TABLE 16. Ranges of Measurement Error Standard Deviations (Operator)

Material Type $\frac{\text { Low }}{\text { Random }}$ Systematic Randor Systematic Random Sys

$\mathrm{UF}_{6}$ Cylinders

$\begin{array}{lllllll}\text { Weighing } & .0001 & .0005 & .00133 & .00163 & .00255 & .00275 \\ \text { Sampling } & .0006 & .00025 & .0017 & .00144 & .0028 & .0026 \\ \text { Analysis } & .0003 & .0002 & .00198 & .00140 & .00265 & .0026\end{array}$

$\mathrm{UO}_{2}$ Powder

$\begin{array}{lllllll}\text { Weighing } & .0005 & .0005 & .00113 & .00113 & .00175 & .00175 \\ \text { Sampling and } & .0007 & .0006 & .00128 & .0012 & .00185 & .0018 \\ \text { Anaiysis } & & & & & & \end{array}$

$\mathrm{UO}_{2}$ Pellets

$\begin{array}{lllllll}\text { Weighing } & .0005 & .0002 & .00113 & .0009 & .00175 & .0016 \\ \begin{array}{c}\text { Sampling and } \\ \text { Analysis }\end{array} & .0006 & .0003 & .0012 & .00098 & .0018 & .00165\end{array}$

$\mathrm{U}_{3} \mathrm{O}_{8}$ Powder

$\begin{array}{lllllll}\text { Weighing } & .0005 & .0005 & .00113 & .00113 & .00175 & .0016 \\ \text { Sampling and } & .0008 & .0008 & .0021 & .0021 & .0034 & .0034\end{array}$

Scrap

$\begin{array}{cllllll}\text { Weighing } & .001 & .0005 & .002 & .00163 & .003 & .00275 \\ \begin{array}{c}\text { Sampling and } \\ \text { Analysis }\end{array} & .01 & .005 & .02 & .0163 & .03 & .0275\end{array}$

Waste

$\begin{array}{lcccccc}\text { Weighing } & 0 & 0 & 0 & 0 & 0 & 0 \\ \text { Sampling and } & .05 & .025 & .0875 & .05625 & .125 & .0875 \\ \text { Analysis } & & & & & & \end{array}$

Miscellaneous

$\begin{array}{lllllll}\text { Weighing } & .0005 & .0002 & .00113 & .0009 & .00175 & .0016\end{array}$

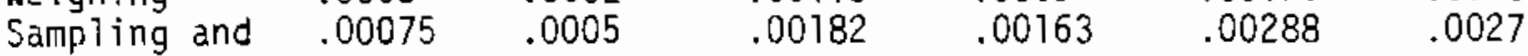

Analysis

$\underline{\text { Rods }}$

$\begin{array}{lllllll}\text { Weighing } & .0001 & .0001 & .00083 & .00083 & .00155 & .0015 \\ \begin{array}{c}\text { Sampling and } \\ \text { Analysis }\end{array} & .0006 & .0003 & .0012 & .0072 & .0018 & .0018\end{array}$


TABLE 17. Ranges of Meàsurement Error Standard Deviations (Inspector)

\begin{tabular}{|c|c|c|c|c|c|c|}
\hline \multirow{2}{*}{ Material Type } & \multicolumn{2}{|c|}{ Low } & \multicolumn{2}{|c|}{ Medium } & \multicolumn{2}{|c|}{ High } \\
\hline & Random & Systematic & Random & Systematic & Random & Systematic \\
\hline \multicolumn{7}{|l|}{$\mathrm{UF}_{6}$ cylinders } \\
\hline $\begin{array}{l}\text { Weighing } \\
\text { Sampling } \\
\text { Analysis }\end{array}$ & $\begin{array}{l}.0001 \\
.0006 \\
.0003\end{array}$ & $\begin{array}{l}.0005 \\
.00025 \\
.0002\end{array}$ & $\begin{array}{l}.00133 \\
.0017 \\
.00148\end{array}$ & $\begin{array}{l}.00163 \\
.00144 \\
.00140\end{array}$ & $\begin{array}{l}.00255 \\
.0028 \\
.00265\end{array}$ & $\begin{array}{l}.00275 \\
.00263 \\
.0026\end{array}$ \\
\hline \multicolumn{7}{|l|}{$\mathrm{UO}_{2}$ Powder } \\
\hline $\begin{array}{l}\text { Weighing } \\
\text { Sampling and } \\
\text { Analysis }\end{array}$ & $\begin{array}{l}.0005 \\
.0006\end{array}$ & $\begin{array}{l}.0005 \\
.0003\end{array}$ & $\begin{array}{l}.00113 \\
.0012\end{array}$ & $\begin{array}{l}.00113 \\
.00098\end{array}$ & $\begin{array}{l}.00175 \\
.0018\end{array}$ & $\begin{array}{l}.00175 \\
.00165\end{array}$ \\
\hline \multicolumn{7}{|l|}{$\mathrm{UO}_{2}$ Pellets } \\
\hline $\begin{array}{l}\text { Weighing } \\
\text { Sampling and } \\
\text { Analysis }\end{array}$ & $\begin{array}{l}.0005 \\
.0006\end{array}$ & $\begin{array}{l}.0002 \\
.0003\end{array}$ & $\begin{array}{l}.00113 \\
.0012\end{array}$ & $\begin{array}{l}.0009 \\
.00098\end{array}$ & $\begin{array}{l}.00125 \\
.0018\end{array}$ & $\begin{array}{l}.0016 \\
.00165\end{array}$ \\
\hline \multicolumn{7}{|l|}{$\mathrm{U}_{2} \mathrm{O}_{8}$ Powder } \\
\hline $\begin{array}{l}\text { Weighing } \\
\text { Sampling and } \\
\text { Analysis }\end{array}$ & $\begin{array}{l}.0005 \\
.0008\end{array}$ & $\begin{array}{l}.0005 \\
.0008\end{array}$ & $\begin{array}{l}.00713 \\
.0021\end{array}$ & $\begin{array}{l}.00113 \\
.0021\end{array}$ & $\begin{array}{l}.00175 \\
.0034\end{array}$ & $\begin{array}{l}.00175 \\
.0034\end{array}$ \\
\hline \multicolumn{7}{|l|}{ Scrap } \\
\hline $\begin{array}{l}\text { Weighing } \\
\text { Sampling and } \\
\text { Analysis }\end{array}$ & $\begin{array}{l}.001 \\
.07\end{array}$ & $\begin{array}{l}.0005 \\
.005\end{array}$ & $\begin{array}{l}.002 \\
.02\end{array}$ & $\begin{array}{l}.00163 \\
.00163\end{array}$ & $\begin{array}{l}.003 \\
.03\end{array}$ & $\begin{array}{l}.00275 \\
.0275\end{array}$ \\
\hline \multicolumn{7}{|l|}{ Waste (NDA) } \\
\hline $\begin{array}{l}\text { Weighing } \\
\text { Sampling and } \\
\text { Analysis }\end{array}$ & $\begin{array}{r}0 \\
.05\end{array}$ & $\begin{array}{c}0 \\
.025\end{array}$ & $\begin{array}{c}0 \\
.0875\end{array}$ & .05625 & $\begin{array}{c}0 \\
.125\end{array}$ & $\begin{array}{c}0 \\
.0275\end{array}$ \\
\hline \multicolumn{7}{|l|}{ Miscellaneous } \\
\hline $\begin{array}{l}\text { Weighing } \\
\text { Sampling and } \\
\text { Analysis }\end{array}$ & $\begin{array}{l}.0005 \\
.00075\end{array}$ & $\begin{array}{l}.0002 \\
.0005\end{array}$ & $\begin{array}{l}.00113 \\
.00182\end{array}$ & $\begin{array}{l}.0009 \\
.00163\end{array}$ & $\begin{array}{l}.00175 \\
.00288\end{array}$ & $\begin{array}{l}.0016 \\
.00275\end{array}$ \\
\hline \multicolumn{7}{|l|}{ Rods (NDA) } \\
\hline $\begin{array}{l}\text { Weighing } \\
\text { Sampling and } \\
\text { Andys is }\end{array}$ & $\begin{array}{c}0 \\
.001\end{array}$ & $\begin{array}{c}0 \\
.001\end{array}$ & $\begin{array}{c}0 \\
.0055\end{array}$ & $\begin{array}{c}0 \\
.0055\end{array}$ & $\begin{array}{r}0 \\
.01\end{array}$ & $\begin{array}{r}0 \\
.01\end{array}$ \\
\hline
\end{tabular}




\section{Crossover Point}

The crossover point, denoted by $\gamma$, is a measure of the sensitivity of the inspector's attributes tests. It is defined as that fraction of the weight of an item such that if more than that fraction were diverted or falsified, the discrepancy would be detected with very high probability; converseiy, if less than the crossover amount were diverted or falsified, the discrepancy might not be detected. Usually, the crossover point is chosen to be $\approx 6$ times the relative standard deviation (combined random and systematic) of the attributes instrument.

We have defined a range of values of the crossover point from $\gamma=.2$ to $\gamma=.4$. With care, the lower end of the range should be attainable in many cases.

\section{Plant Size (Annua? Throughput)}

Table 18 contains a breakdown by size of LEU conversion-fabrication plants. The figures shown are approximate. The data are taken from a paper presented at a recent advisory group meeting. For this study, we have established the range of interest as 200-600 tons of uranium.

Summary of the Ranges of Input Factors Considered

Table 19 summarizes the ranges of interest for the factors. Note that the three levels are "evenly spaced", i.e., the middle values are exactly halfway between the high and low values. This is convenient for purposes of the experimental design. It is for this reason that the middie values of $\alpha$ and $\beta$ are .055 instead of the more conventional .05 .

\section{Experimental Design}

In order to study the effects of the input factors over the region of interest to us, we seiected an experimental design called a Box-Behnken design with seven input factors. This design permits an assessment of:

- the direction (positive or negative) of the effect of each factor

- the magnitude of the effect of each factor 
TABLE 18. LEU Conversion-Fabrication Plants Under IAEA Safeguards (November 1979)

10 Conversion-Fabrication Plants

1 Conversion Plant

"Some" Small Pilot Plants

CAPACITY

NO. OF PLANTS

50
100
$200-250$
$400-600$
1000

1

3

2

1000

3

1

TABLE 19. Summary of Ranges of Input Factors

\begin{tabular}{|c|c|c|c|}
\hline & Low & Middle & $\underline{\text { High }}$ \\
\hline False Alarm Probability & .01 & .055 & .7 \\
\hline Non-detection Probability & .01 & .055 & .7 \\
\hline Detection Goal Quantity ( $\mathrm{kg} U)$ & 833 & 2500 & 4166 \\
\hline $\begin{array}{l}\text { Operator's Measurement } \\
\text { Capability }\end{array}$ & low & middle & high \\
\hline $\begin{array}{l}\text { Inspector's Measurement } \\
\text { Capability }\end{array}$ & low & middle & high \\
\hline Crossover Point & .2 & .3 & .4 \\
\hline Plant Size (Tons Throughput) & 200 & 400 & 600 \\
\hline
\end{tabular}


- the curvature or non-linearity of the effects as

a function of the factors

- interactions between factors

The Box-Behnken designs are response surface designs, well-suited to the application of regression techniques to fit response surfaces or simple empirical models to the data.

The complete layout of the seven variable Box-Behnken design is shown in Table 20. There are 62 rows in the table. Each row represents a case or an experimental run. There are seven columns, one for each input factor. The entries in the Table denote the levels of each factor: the plusses denote the high vaiues for each factor; the zeroes denote the middle values; and the minuses denote the low values.

To 11 lustrate the use of the table, consider the following example. Take the first row, which contains the following entries:

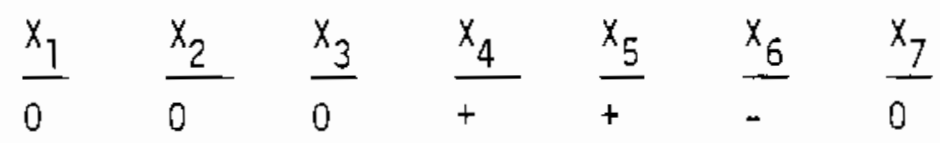

The columns are defined as follows:

$$
\begin{aligned}
& x_{1}=\text { false alarm probability } \\
& x_{2}=\text { non-detection probability } \\
& x_{3}=\text { detection goal quantity } \\
& x_{4}=\text { operator's measurement capability } \\
& x_{5}=\text { inspector's measurement capability } \\
& x_{6}=\text { crossover point } \\
& x_{7}=\text { plant size (annual throughput) }
\end{aligned}
$$

For this particular run or case, one would set the false alarm probability at its middle value, .055 , the non-detection probability at its middle value, .055 , the goal quantity at its middle value, $2500 \mathrm{~kg}$, the operator's measurement capability at its high level (large measurement errors), the inspector's measurement capability also at its high level (large measurement errors), 
TABLE 20. Seven Variable Box-Behnken Design (8)

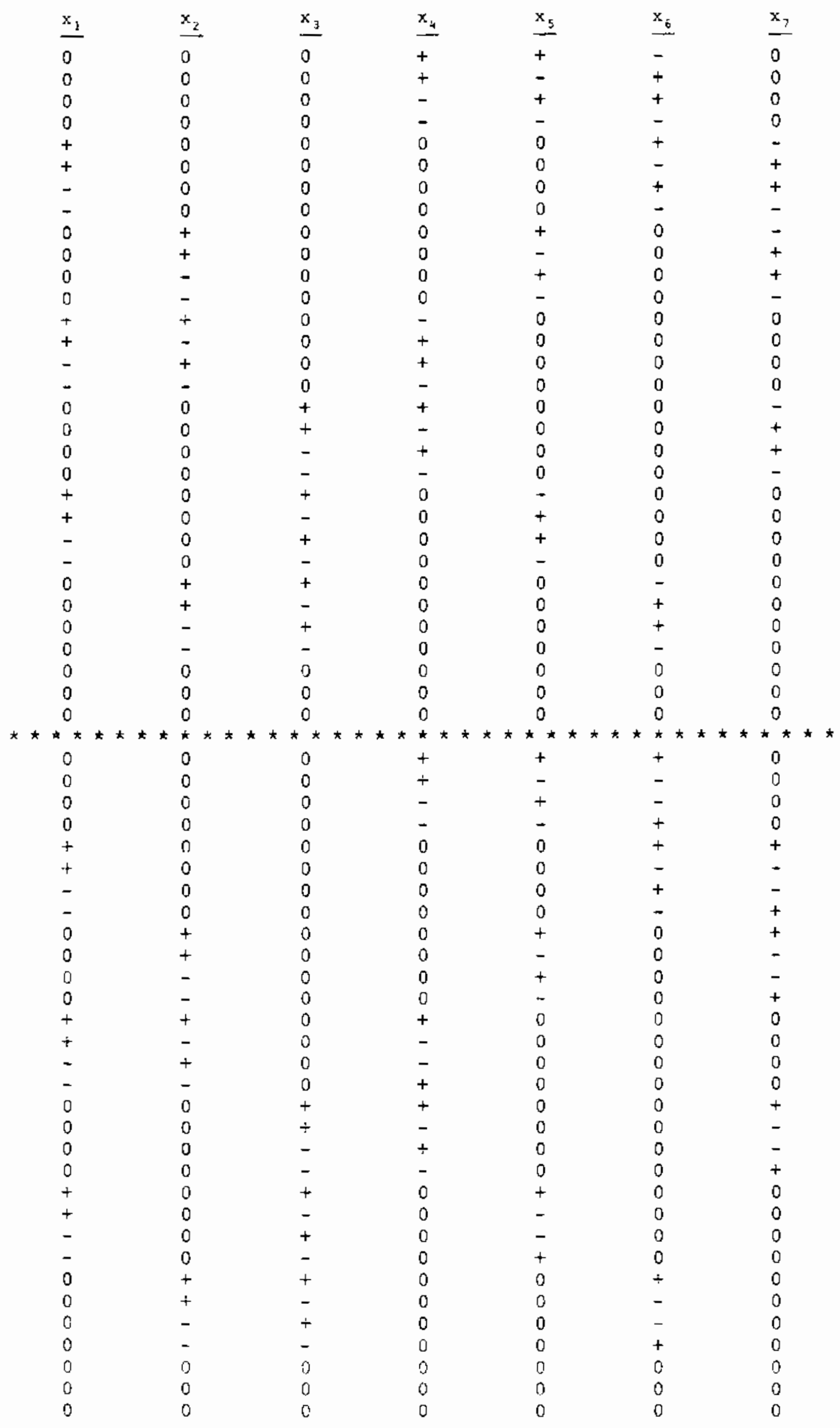


the crossover point at its low value, .2, and the plant size at its middle value, 400 tons of uranium throughput per year.

For each of the sixty-two cases, we used one of the rows in Table 20 to establish the values of the input factors. We then calculated the values of the responses corresponding to the specified levels of the input factors. The INSPECT programs were used extensively to facilitate the calculations.

The resuits can be written in the form of a large table of data. Each row in the table represents one case. The first seven columns contain the values of the input factors. The remaining columns denote the values of the responses. Table 21 contains the data for the 62 cases we considered. 


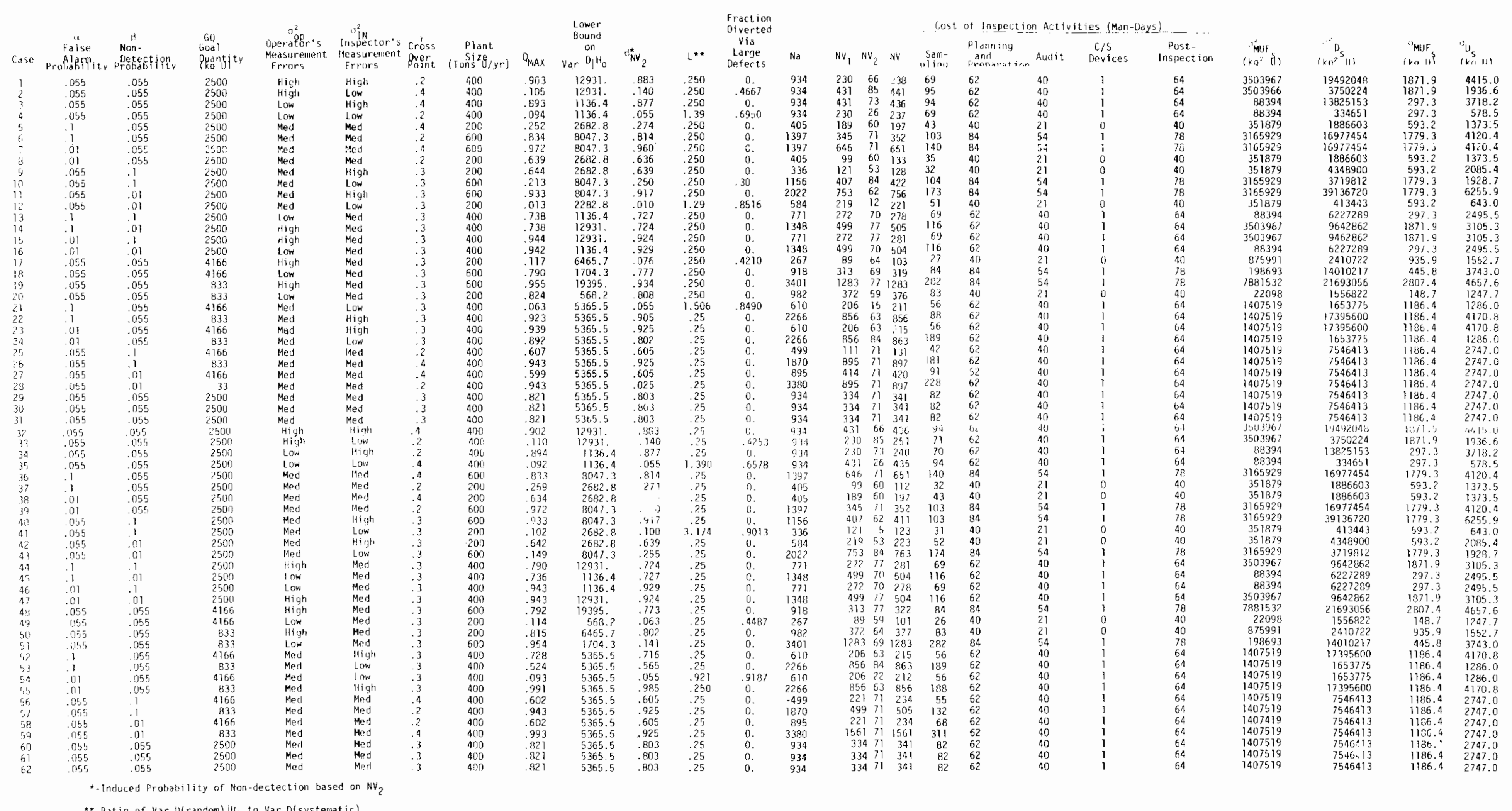




\section{ANALYSIS}

In analyzing the data generated with the experimental design, we take the following approach. First, we describe briefly how to set up the raw data (as shown in Table 21) for the analysis. Second, we take a look at the characteristics of the Box-Behnken design that we used in the study. This involves various plots (histograns and scatter plots) of the input data. Third, we examine graphically (again using histograms and scatter plots) the behavior of the response variables. Fourth, we fit response surfaces (regression analysis) to the data and examine the fitted equations.

\section{Setting Up the Data}

Before beginning the analysis, it is first necessary to put the data in a convenient form. In doing $\$ 0$, there are several things to consider. First, we want to use standard statistical software to facilitate the analysis. Second, we want to plot the data in various ways to help make the resuits understandable. Third, we uitimately want to iit response surfaces to the data.

The software used for the analysis is the BMDP package, (2) which is avai]able to the IAEA in Vienna. The input data requirements for BMDP are fajrly straightforward. The formats are standard Fortran. BMDP can be used to plot the data (iine-printer plots have sufficient resolution for our purposes). One other point should be noted. It is often useful to perform the statistical araiysis using the coded input data (low values = -1 , medium values $=0$, and high values $=1$ ) rather than the original uncoded values. In the analyses that follow we will give some examples in which the data are coded, and others in which the data are not coded.

In order to fit response surfaces it is necessary to augment the data set to include all of the predictor or input variables that we will need. The data in Table 21 only contain the seven first order input variables, narrely, false alarm probability, nondetection probability, detection goal quantity, operator's measurement capability, inspector's measirement capability, 
crossover point, and throughput. For response surface analysis we also need second order terms: squared terms such as false alarm probability squared or goal quantity squared, and interaction or cross-product terms such as goal quantity times throughput. It will become clear in later sections how these second order terms are used. We used the BMDP programs to transform the first order inputs and produce the augmented data set containing squared and interaction terms.

\section{Characteristics of the Box-Behnken Experimenta] Design}

Before undertaking the formal analysis of the results, it is interesting to take a quick look at some of the characteristics of the seven variable Box-Behnken design used for the study. In selecting the Box-Behnken design for the sensitivity study, we intentionally chose a design that has good properties for response surface analyses. In particular, it is a three level design which is a subset of the full three level factorial and it permits us to assess first order effects and second order effects (squared terms and interactions). Good experimental design is usually termed "bold". Boldness means:

- the experimental points are widely spread out over the region of interest,

- the experimental points are balanced and symmetrical,

- the input variables are as uncorrelated as possible.

We shall now examine the Box-Behnken design from this point of view.

Figure 5 shows an example of a first order input variable, the false alarm probability. Figures for the other input variables are shown in Appendix $B$. The pattern of balance and symetry is the same for all seven first order input variables. The data shown is coded, that is, it has the values of $-1,0$, and +1 . These correspond to the false alarm rates of $1 \%$, $5.5 \%$, and $10 \%$ respectively. The balance is obvious as there are 12 low values, 12 high values, and 38 middle values. 


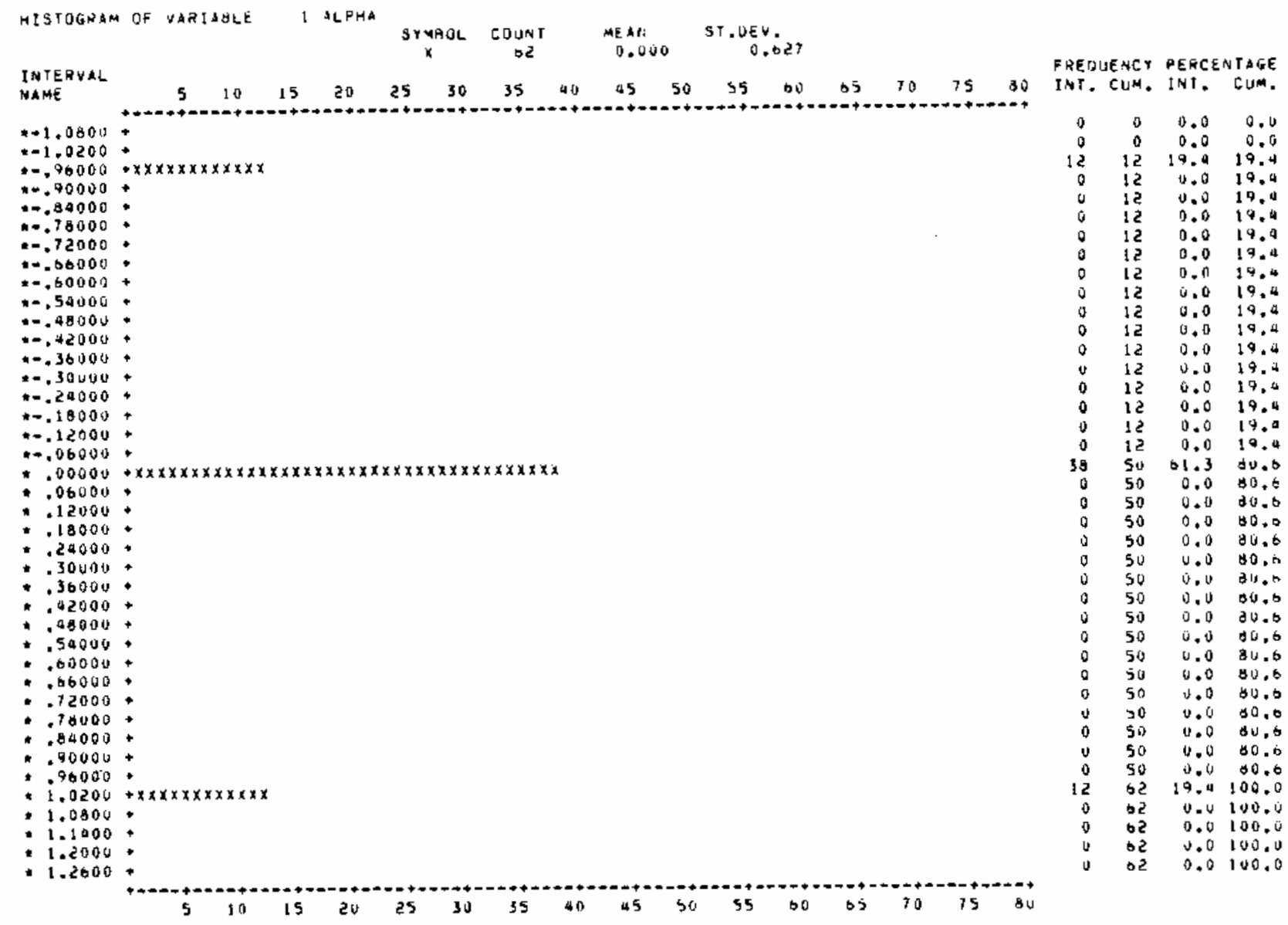

FIGURE 5. Histogram of Alpha (False Alarm Probability) 
Figure 6 shows an input variable squared term. The remainder are located in Appendix B. Again they a1] follow the same pattern, as they must because the first order terms did. Note that there are only two levels here instead of three as there were for the first order terms. This is because the low value (-1) squared is the same as the high value (1) squared.

Figure 7 shows an example of a cross-product or interactive term invoiving alpha, the faise alarm probability. The remaining histograms of crossproduct terms involving alpha are shown in Appendix B. All the histograms for interaction terms follow the same pattern with four cases at the low value, four at the high value, and the remaining 54 cases at the middle value.

Clearly, the Box-Behnken design has characteristics of balance and symmetry as far as the levels of the predictor variables are concerned.

The next concern is that of correlations between input variables. To look at possible correlations between input variables, we can examine scatter plots of the variables, one versus another, and also calculate correlation coefficients between pairs of variables.

Figures 8 and 9 are scatter plots of aipha, the false alarm probability, versus other first order inputs. There are two basic patterns. Figure 8 is a plot of alpha versus itself. The result is obviously a straight line with slope $=1$ since a variable is always perfectly correlated with itself. Figure 9 shows alpha versus beta, the non-detection probability. Plots of alpha versus the other first order inputs are inciuded in Appendix $B$. The same pattern is evident in all the plots. There are two cases in each of the "corners" of the experimental region, eight cases on each of the four sides, and the remaining 22 cases at the center point. The variables are uncorrelated (this is the meaning of $\mathrm{COR}=.000$ printed under the plot) and the experimental pattern is obviously balanced and symmetric. The symmetry is actually circular about the center point although this is perhaps not obvious from the plot. (The corners, each having two points, are equidistant from the center point; the sides, each having eight points, are also equidistant from the center point.) This is characteristic of Box-Behnken designs, i.e., they are "rotatable." 


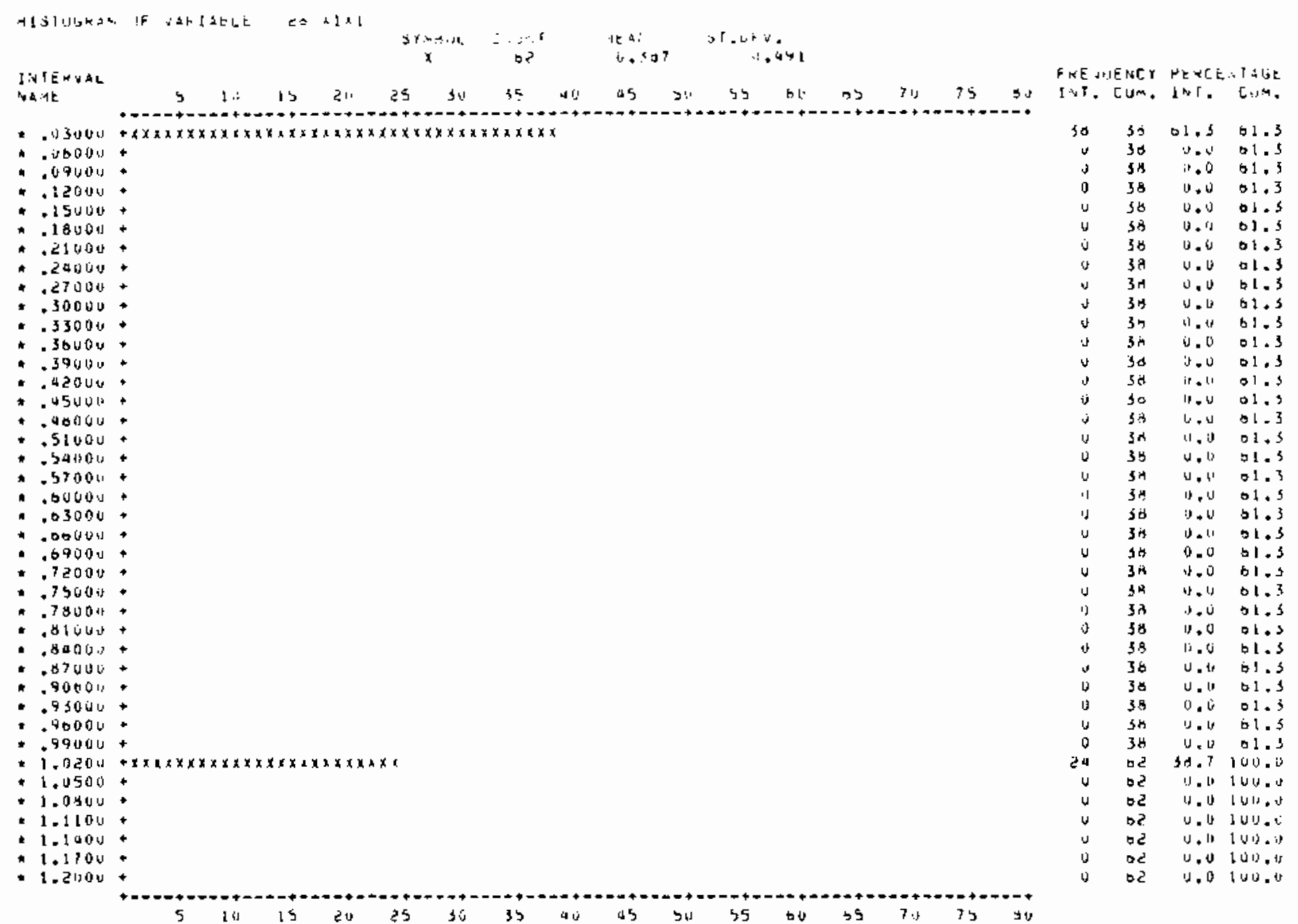

FIGURE 6. Histogram of XIX1 (Alpha Squared) 


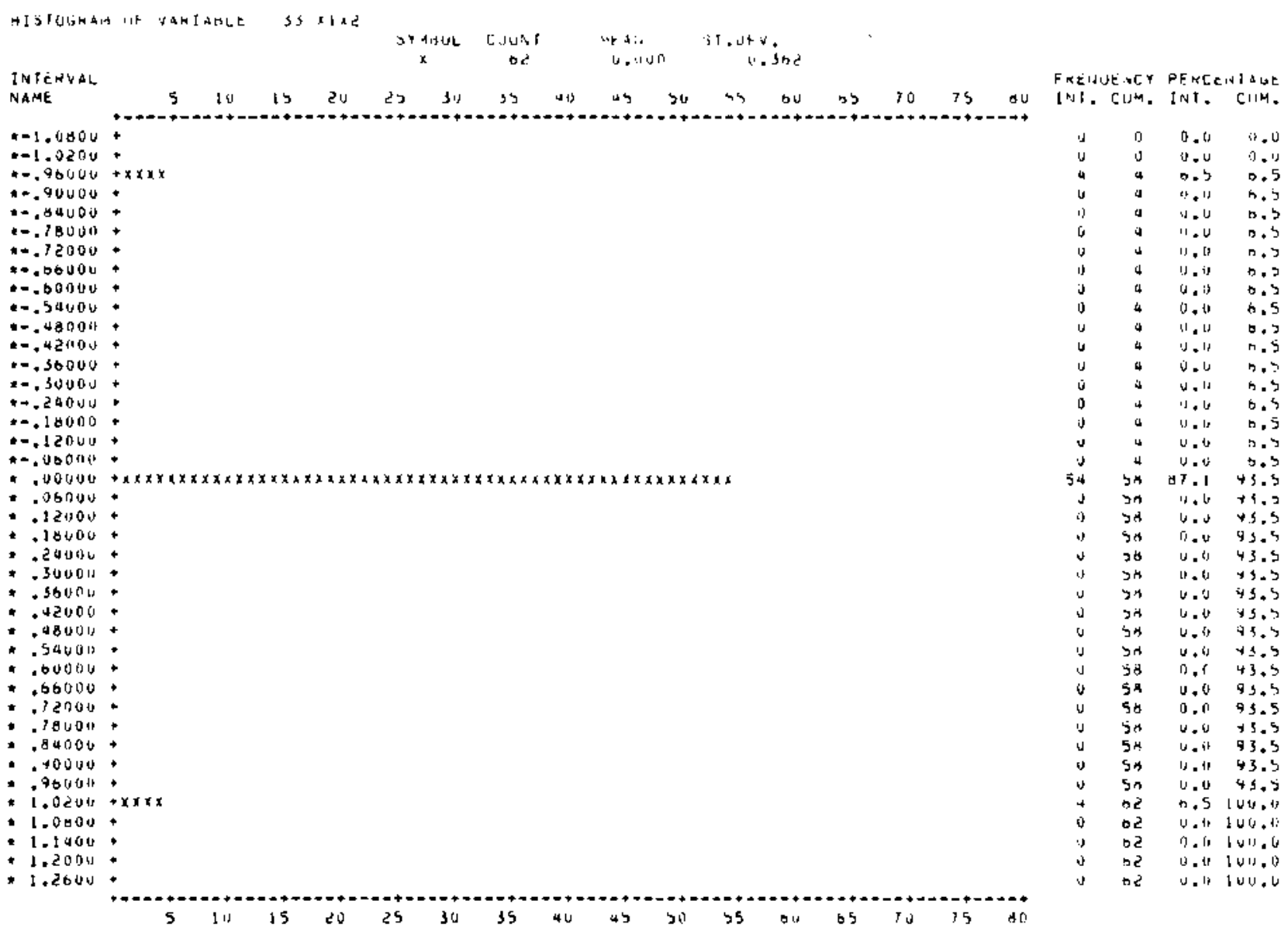

\section{FIGURE 7. Histogram of $X 7 \times 2$ (ATpha Times Beta)}




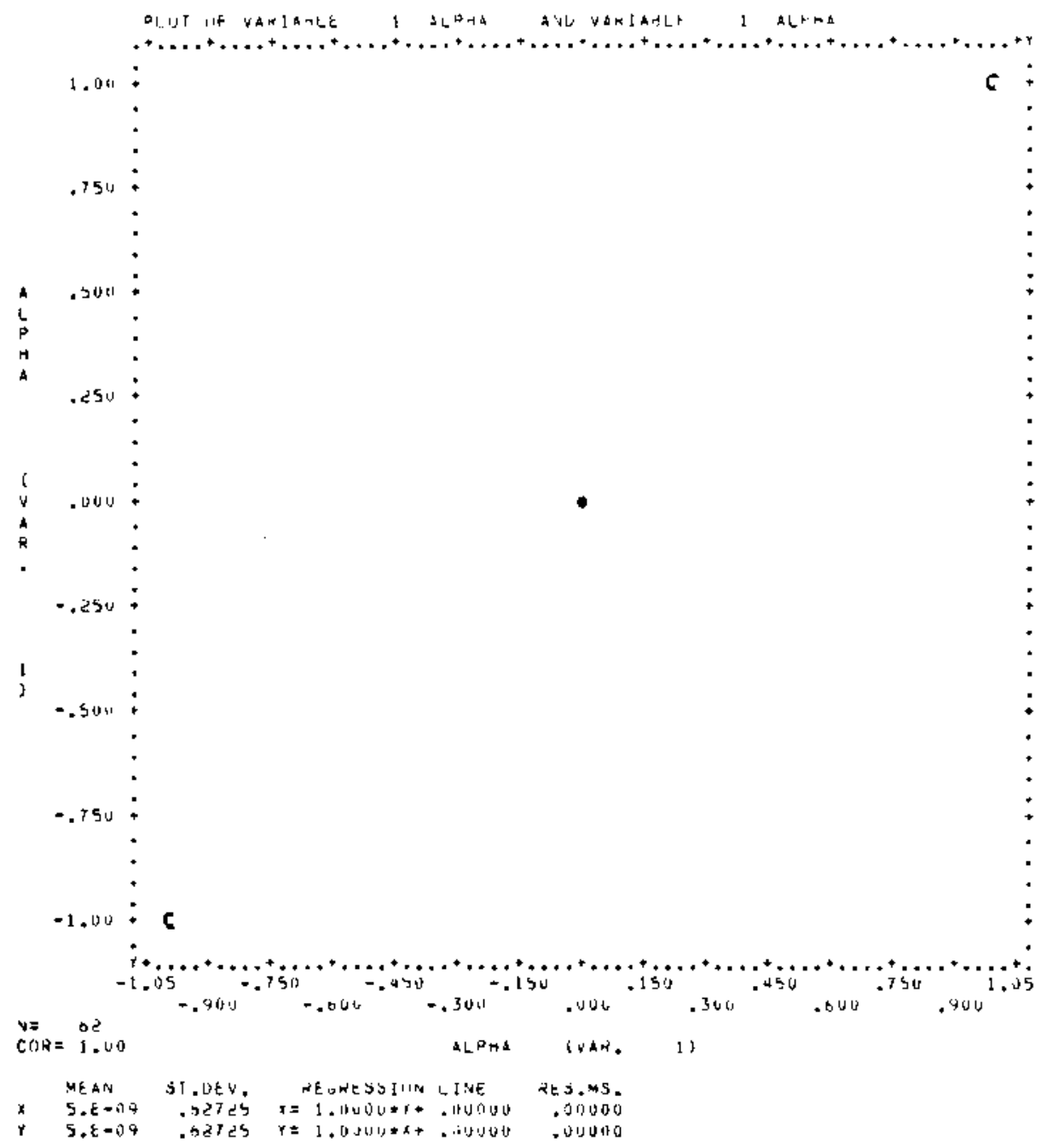

FIGURE 8. Alpha Versus Alpha 


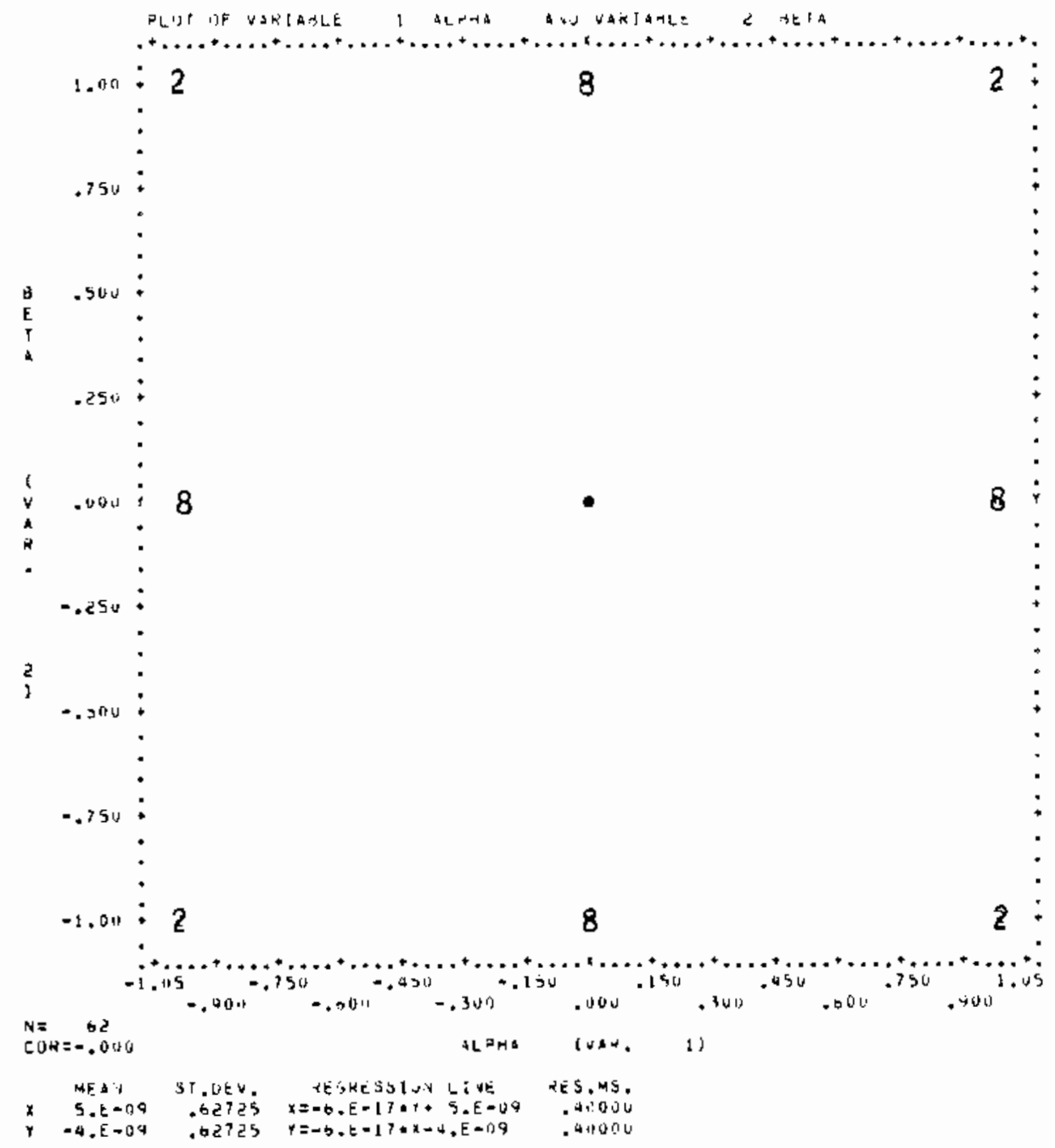

FIGURE 9. Beta Versus Alpha 
All the other plots of first order inputs versus other first order inputs follow the same pattern. They have not been included in this report.

Figure 10 shows a plot of alpha vs. alpha squared. Again the correlation coefficient is zero. There are 12 points where alpha = alpha squared $=1,12$ points where alpha $=-1$ and alpha squared $=1,38$ points where alpha $=$ alpha squared $=0$. All other plots of the form $x$ vs. $x^{2}$, where $x$ is a first order input, follow the same pattern.

Figure 11 shows a piot of beta vs. alpha squared. Again, the correlation is zero and the points are balanced. All other plots of the form $x_{i}$ vs. $x_{j}^{2}$, where $x_{i}$ and $x_{j}$ are first order inputs and $i \neq j$, follow this pattern.

Figure 12 shows a plot of beta squared vs. alpha squared. This correlation coefficient is no longer zero and the plot no longer has the balanced appearance that earlier ones had. There are eight points at $(1,1), 16$ at $(0,1), 16$ at $(1,0)$, and 22 at $(0,0)$. The squared terms are correlated, in other words, although the correlation is not high.

The reasons for the interesting geometry of the experimental design are rather complicated. A detailed discussion of the subject may be found in Box and Hunter (1957). For the purposes of this study it is sufficient to note that the Box-Behnken designs are good designs for fitting second order polynomials (response surfaces) to the data. 


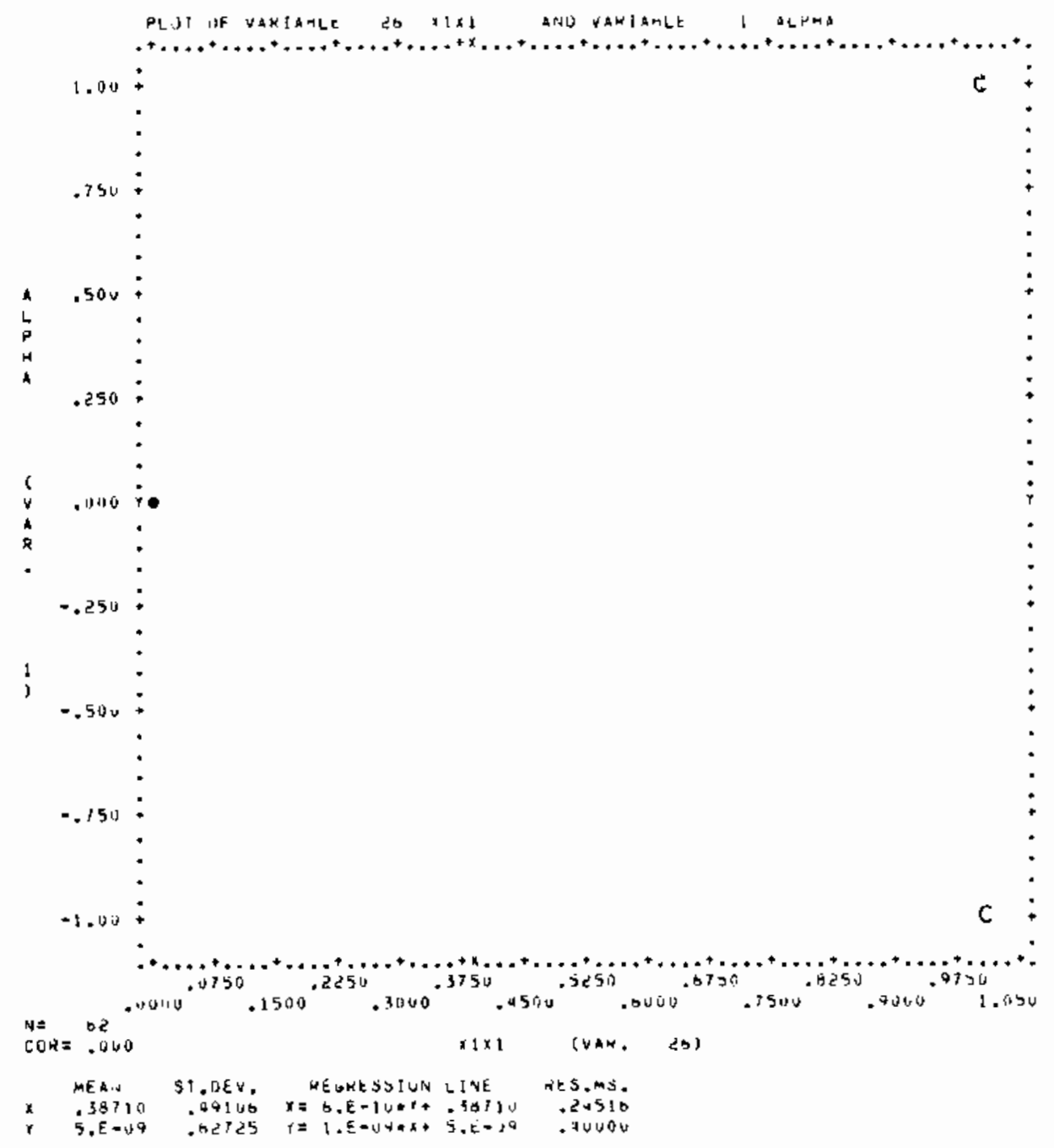

FIGURE 10. A7pha Versus A1pha Squared $(X] \times 1)$ 


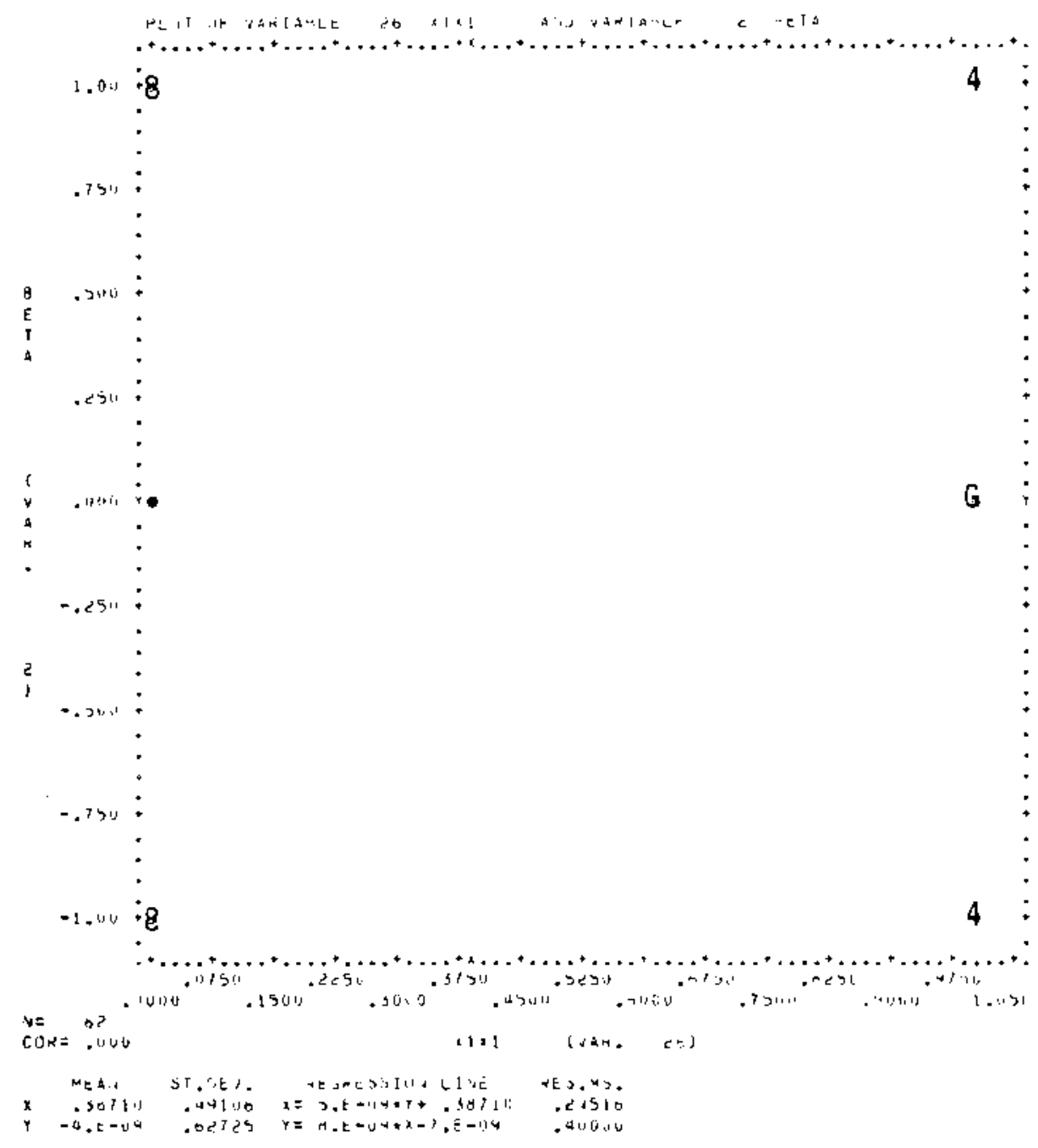

FIGURE 11. Beta Versus A]pha Squared $(X 1 \times 1)$ 


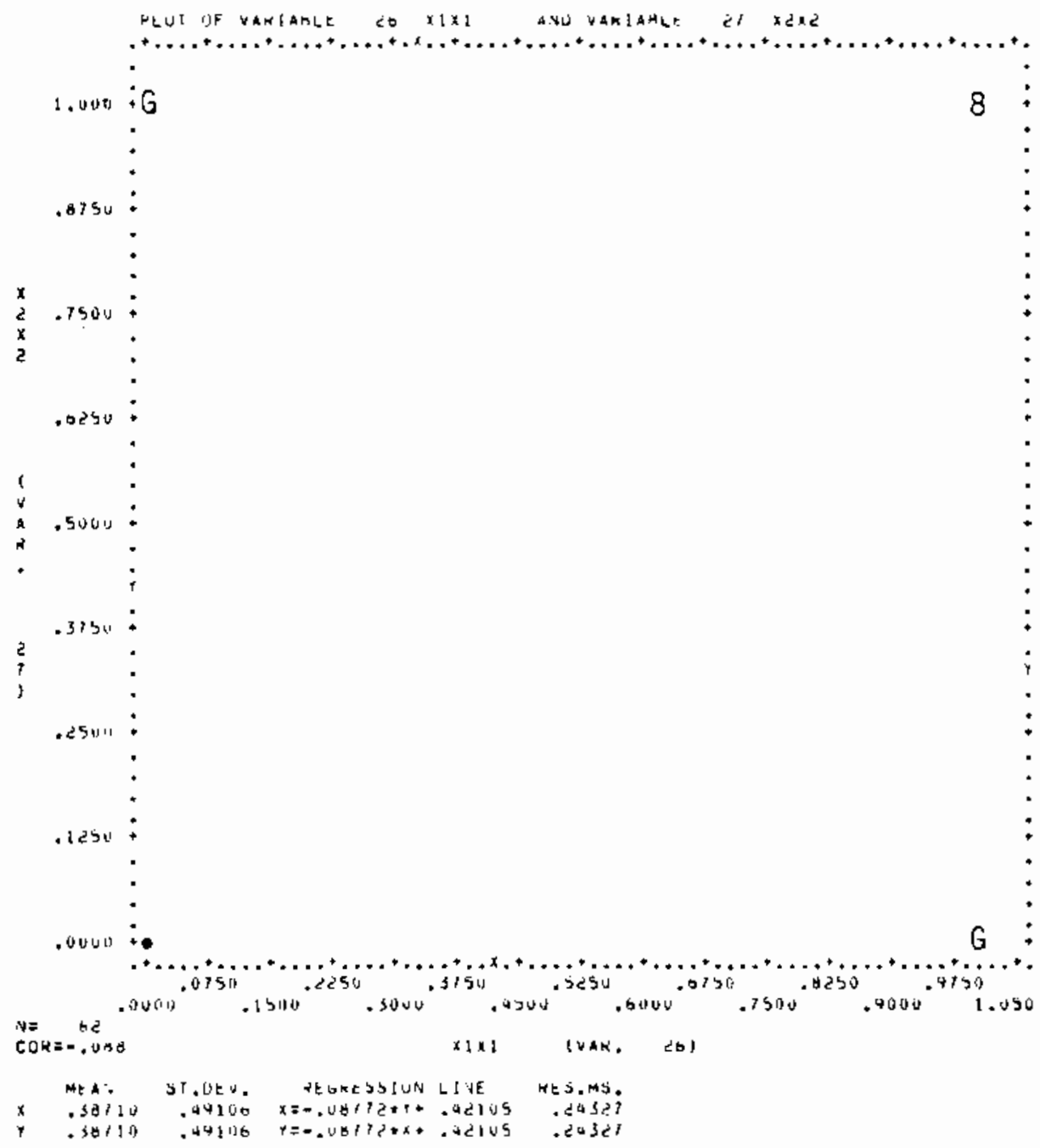

FIGURE 12. Beta Squared $\left(X_{2} \times 2\right)$ versus Alpha Squared $(X 1 \times 1)$ 
Behavior of the Response Variables

In this section, we use graphical and regression techniques to examine some aspects of the behavior of the response variables. In performing the analysis, we considered the 19 response variables listed in Table 22 . The list is by no means exhaustive; other responses could be considered as well. For this report, we confine the discussion to a few of the most important responses.

\section{TABLE 22 List of Nineteen Response Variables}

$Q_{\text {MAX }}$ - The probability of nondetection assuming the diverter's optimal strategy

Systematic Error Variance of MUF

Systematic Error Standard Deviation of MUF

Systematic Error Variance of the $\hat{D}$ Statistic

Systematic Error Standard Deviation of the $\hat{O}$ Statistic

Attributes Sample Size

Sample Size for Variables Measurements to Detect Partial Defects

Sample Size for Variables Measurements to Detect Bias

Total Variables Sample Size

Cost of Planning and Preparing for an Inspection

Cost of Audit Activities

Cost of Material Verification

Cost of Containment and Surveillance Activities

Cost of Post Inspection Activities

Total Inspection Cost (Man-days)

Fraction of the Goal Quantity Diverted via Large Defects Assuming the Diverter's Optimal Strategy

Lower Bound on the Random Error Variance of the D Statistic

Probability of Nondetection Assuming the Entire Goal Quantity is Diverted via the MUF-D̂ Path and the Variables Sample Size is Governed by the Need for Variables Measurements to Detect Bias

Ratio of the Random Error Variance of $\hat{D}$ (Under the Alternative Hypothesis) to the Systematic Error Variance of $\hat{D}$ 
$Q_{\text {MAX }}$. $Q_{\text {MAX }}$ is defined as the probability of nondetection assuming the diverter's optimal strategy. 1-Q $Q_{\text {MAX }}$ is the probability of detection. Q MAX is a prime index of safeguards system performance.

Figure 13 is a histogram of the values of $Q_{\operatorname{MAX}}$ recorded for the 62 cases inciuded in the experimental design. It can be seen that $Q_{\text {MAX }}$ spans the entire range from 0 to 1 . One unusual feature of the data is that it seems to fall into two main groups with a gap in between them. The first group, at the top of the graph, ranges from $Q_{\operatorname{MAX}} \equiv 0$ to $Q_{\text {MAX }}=0.27$. These are cases for which the detection probability $\left(1-Q_{\text {MAX }}\right)$ is fairly high. The other group, ranging from $Q_{\operatorname{MAX}} \cong 0.54$ to $Q_{\operatorname{MAX}} \cong 1$ represents low detection probabilities. The reasons for this grouping will become clear when we examine the relationship between $Q_{\text {MAX }}$ and the inspector's measurement capability.

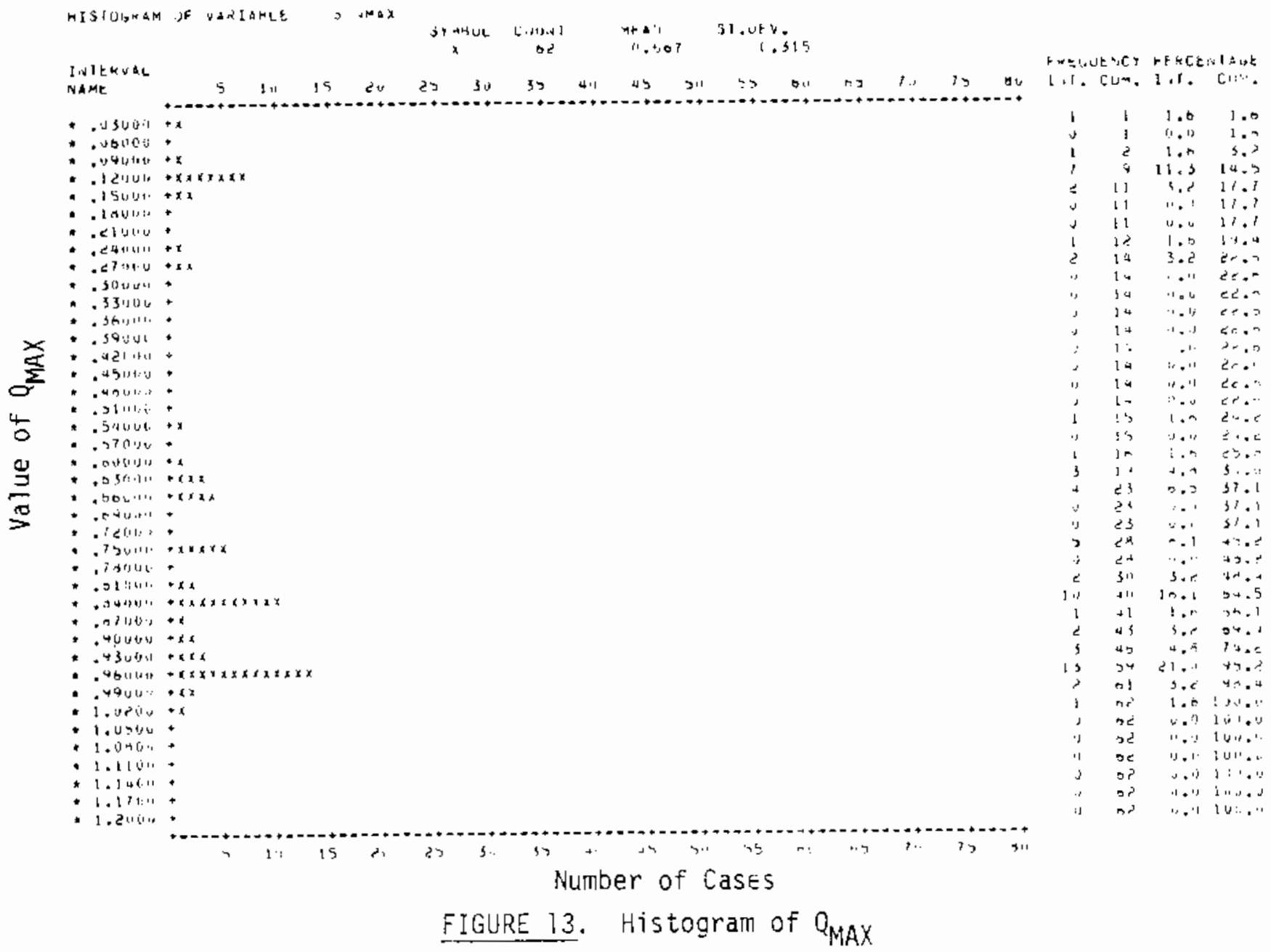


Figure 14 shows a scatter piot of $Q_{\text {MAX }}$ versus aipha. Figure 15 shows histograms of $0_{\text {Max }}$ for each of the three levels of alpha. Visually, the plots illustrate the fact that alpha by itself does not explain very much of the variation in $Q_{\text {MAX }}$ as there is a large scatter in $Q_{\text {MAX }}$ for each value of alpha. However, the regression calculation shows that there is a small negative correlation. As alpha increases, Q MAX decreases sightly. This is consistent with the well known observation that one can achieve an improved detection probability (smaller $0_{M A X}$ ) if one is willing to accept a higher false alarm probability in return. It is clear, however, that the effect is small over the range of alpha values considered $(\alpha=.01$ to $\alpha=.1)$.

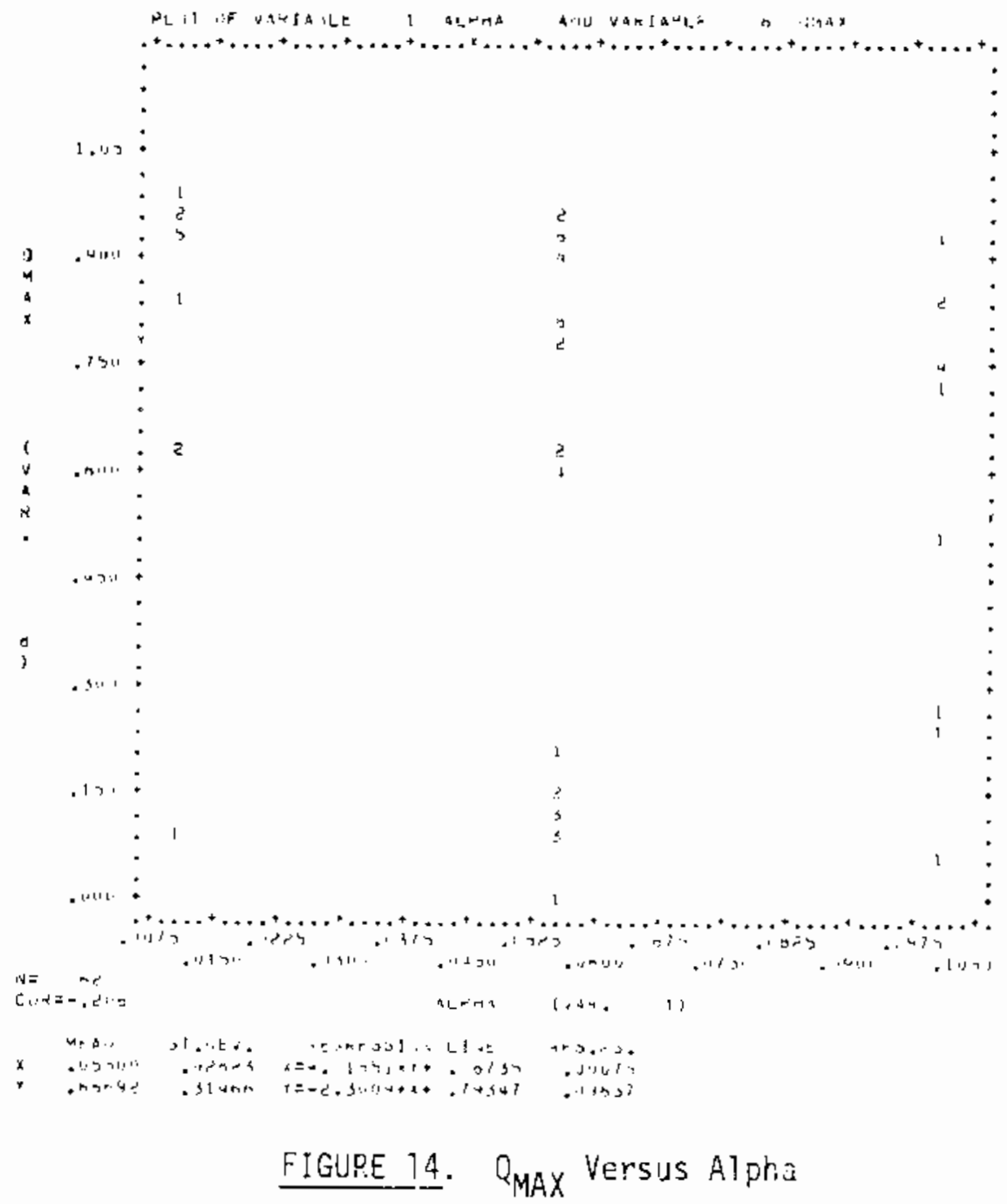




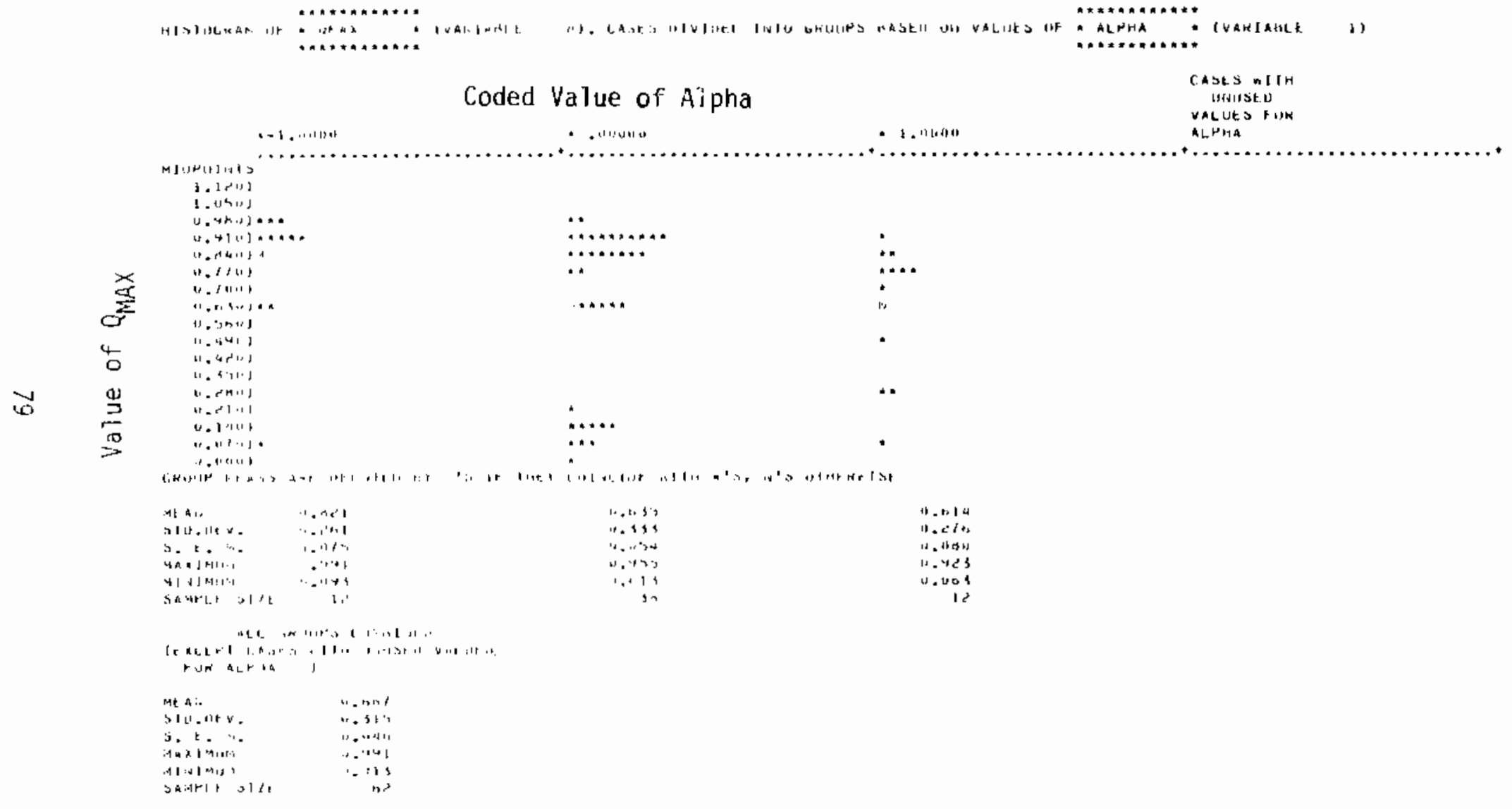

FIGURE 15. Histograms of $Q_{\text {MAX }}$ Versus Alpha 
In contrast to Figures 14 and 15 , a variable with a noticeable effect on $Q_{\text {MAX }}$ is the inspector's measurement capability. Figure 16 shows a piot of $Q_{\text {MAX }}$ versus the inspector's measurement capability (denoted by MCAPINSP in the figure). Visualiy, there appears to be a clear relationship; however, there is still a lot of scatter in the data. The inspector's measurement capability appears to have a strong effect but it is clear that other variables are important as well. The relationship can be expressed in the following way: as the quality of the inspector's measurements decreases $(-1$ denotes good measurement capability, +1 denotes poor measurement capability), the probability of nondetection tends to increase, and the probability of detection to decrease.

Figure 17 shows the same thing in a different way. Histograms of the values of $Q_{\text {MAX }}$ for each of the three levels of inspector measurement capability are plotted side by side. The relationship is quite striking, and it recalls Figure 13, which was the histogram of all the $Q_{\text {MAX }}$ values together. The low values of $Q_{\text {MAX }}$ are almost all cases for which the inspector's measurement capability was very good (MCAPINSP $=-1$ ). The reason for the two clumps of data points in Figure 13 is now clear: the high probability of detection is made possible mainly by the high quality of the inspector's verification measurements in those cases.

Graphs plotting $Q_{\text {MAX }}$ against the other input variables are shown in Appendix C. 


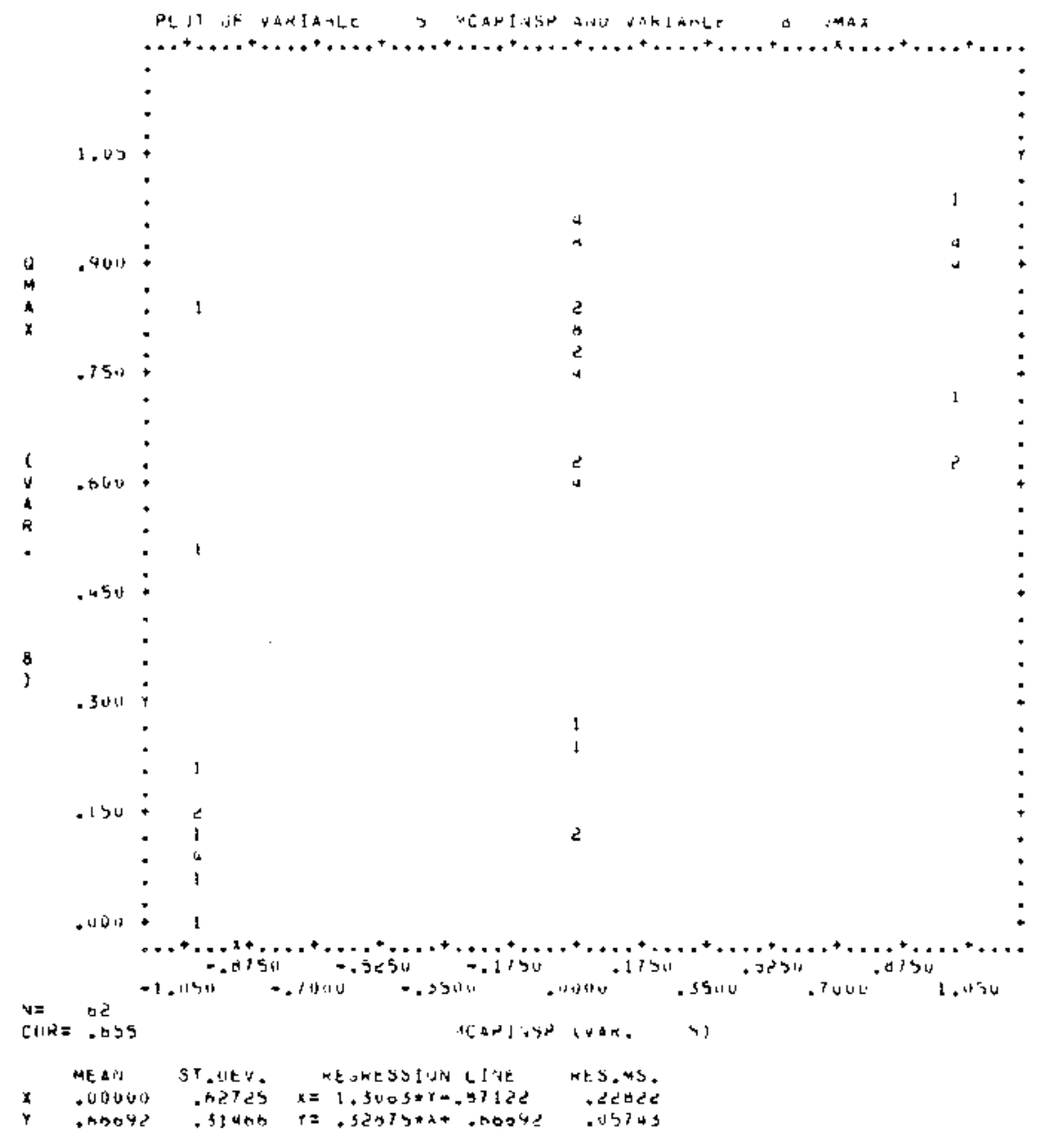

FIGURE 16. Q QMAX Versus Inspector's Measurement Capabitity 


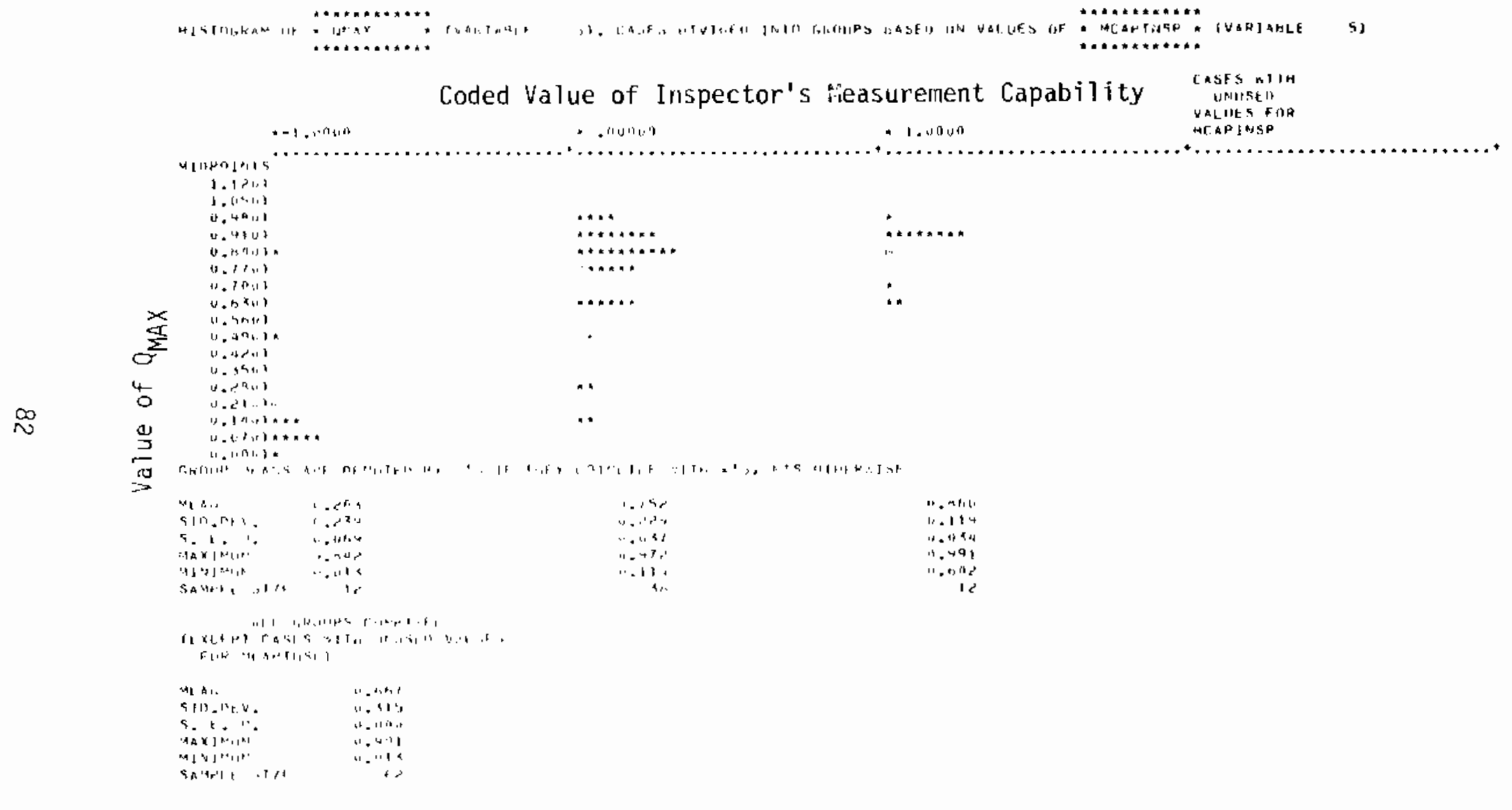

FIGURE 17. Histograms of $Q_{\text {MAX }}$ Versus Inspector's Measurement Capability 
To supplement the graphical analysis as was just shown, there was considerable use of regression analysis. Table 23 shows a summary of the regression analysis of $Q_{M A X}$ as a function of all the input variabies. The "MULTIPLE R-SQUARE," also known as the coefficient of multiple determination, is about .97. This means that approximately $97 \%$ of the variation in $Q_{M A X}$ is accounted for or explained by the fitted curve. The column of primary interest in the table is headed "Coefficient." The variables with large (in absolute value) regression coefficients are the ones that have the strongest influence on $Q_{\text {MAX. }}$.

A word on the notation used in the table: the variables denoted by $X[X 1$ and similar codes are second order variables. $X] X]$ is variable 1 , or alpha, squared. $\times 3 \times 7$ is variable 3 times variable 7 or goal quantity times throughput.

The largest coefficient in absolute value is associated with the inspector's measurement capability (MCAPINSP). In Table 24 , the regression coefficients of the variables involving the inspector's measurement capability are summarized. From the table, it is apparent that $Q_{\text {MAX }}$ has an important nonlinear (specifically quadratic) dependence on the inspector's measurement capability: the coefficient of $\times 5 \times 5$, which is the squared term involving inspector's measurement capability, is larger in absolute value than most of the first order coefficients. Some important interactions can also be seen: the coefficient of $\times 3 \times 5$ is .12037, which is relatively large. This means that there is an interaction between $\times 3$, goal quantity, and $x 5$, the inspector's measurement capability; the effect of each depends on the level of the other.

Table 25 shows the coefficients involving the throughput $(x 7)$. The linear term (THRUPUT), the squared term $(X 7 \times 7)$, and the interaction between throughput and goal quantity $(\times 3 \times 7)$ are all relatively large. 
TABLE 23. Summary of Regression Analysis: QMAX

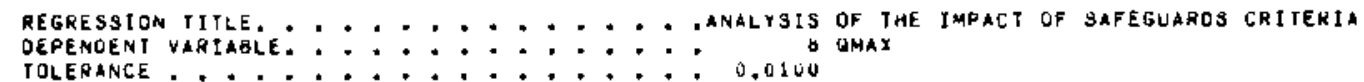

$\begin{array}{ll}0.000 & 1.000000 \\ 0.089 & 1.000004 \\ 0.000 & 1.000000 \\ 0.043 & 1.000000 \\ 0.000 & 1.000000 \\ 0.991 & 1.000000 \\ 0.000 & 1.000000 \\ 0.071 & 0.917763 \\ 0.965 & 0.917763 \\ 0.738 & 0.917763 \\ 0.287 & 0.917763 \\ 0.000 & 0.917763 \\ 0.021 & 0.917763 \\ 0.000 & 0.917763 \\ 0.990 & 1.000000 \\ 0.555 & 1.000000 \\ 0.997 & 1.000000 \\ 0.778 & 1.000000 \\ 6.990 & 1.000004 \\ 0.056 & 1.000000 \\ 0.974 & 1.000000 \\ 0.991 & 1.000000 \\ 0.539 & 1.000000 \\ 0.993 & 1.000000 \\ 0.912 & 1.000000 \\ 0.958 & 1.000000 \\ 0.001 & 1.000000 \\ 0.974 & 1.000000 \\ 0.004 & 1.000000 \\ 0.851 & 1.000000 \\ 0.896 & 1.000000 \\ 0.971 & 1.000000 \\ 0.903 & 1.000000 \\ 0.102 & 1.000000 \\ 0.964 & 1.000000\end{array}$


TABLE 24. Coefficients Involving Inspector's

Measurement Capability

$\begin{array}{lrr}\text { Variable } & & \text { Coefficient } \\ \text { MCAPINSP } & & .32875 \\ \times 1 \times 5 & & .00862 \\ \times 2 \times 5 & & -.07888 \\ \times 3 \times 5 & & .12037 \\ \times 4 \times 5 & & -.00575 \\ \times 5 \times 5 & & -.23498 \\ \times 5 \times 6 & & -.00375 \\ \times 5 \times 7 & & .04163\end{array}$

TABLE 25. Coefficients Involving Throughput

$\begin{array}{lrl}\text { Variable } & & \text { Coefficient } \\ \text { THRUPUT } & & .17813 \\ \times 1 \times 7 & .06063 \\ \times 2 \times 7 & & -.00337 \\ \times 3 \times 7 & .13513 \\ \times 4 \times 7 & & .00173 \\ \times 5 \times 7 & & .04163 \\ \times 6 \times 7 & .00137 \\ \times 7 \times 7 & -.13342\end{array}$


Table 26 shows the coefficients invoiving the goal quantity (GQ or X3). The linear term is important and the interactions between goal quantity and throughput ( $\times 3 \times 7)$ and between goal quantity and inspector's measurement capability $(\times 3 \times 5)$ are aiso relatively large.

TABLE 26. Coefficients Involving Goal Quantity

$\begin{array}{lll}\text { Variable } & & \text { Coefficient } \\ \text { GQ } & & -.18975 \\ \times 1 \times 3 & & .01813 \\ \times 2 \times 3 & & .00100 \\ \times 3 \times 3 & & .00790 \\ \times 3 \times 4 & & .00162 \\ \times 3 \times 5 & & .12037 \\ \times 3 \times 6 & & -.00100 \\ \times 3 \times 7 & & .13513\end{array}$

Tables 27 through 30 contain the regression coefficients involving the false alarm probability, alpha, the goal nondetection probability, beta, the operator's measurement capability, MCAPOP, and the crossover point, gamma. All of the coefficients are relatively small, indicating that these variables tend to have a smaller impact on $\mathrm{M}_{\text {MaX }}$ than do the throughput, the goal quantity, and the inspector's measurement capability.

TABLE 27. Coefficients Involving the False Aiarm Probability, Alpha

\begin{tabular}{lll} 
Variable & & Coefficient \\
\hline ALPHA & & -.70354 \\
$\times 1 \times 1$ & & .04396 \\
$\times 1 \times 2$ & & .00038 \\
$\times 1 \times 3$ & & .01813 \\
$\times 1 \times 4$ & & .00012 \\
$\times 1 \times 5$ & & .00862 \\
$\times 1 \times 6$ & & -.00037 \\
$\times 1 \times 7$ & & .06063
\end{tabular}


TABLE 28. Coefficients Involving the Goal Nondetection Probability, Beta

\begin{tabular}{|c|c|}
\hline Variable & Coefficient \\
\hline BETA & .00708 \\
\hline$\times] \times 2$ & .00038 \\
\hline$\times 2 \times 2$ & .00102 \\
\hline$\times 2 \times 3$ & .00100 \\
\hline$\times 2 \times 4$ & .00013 \\
\hline$\times 2 \times 5$ & -.01888 \\
\hline$\times 2 \times 6$ & -.00025 \\
\hline$\times 2 \times 7$ & -.00337 \\
\hline
\end{tabular}

TABLE 29. Coefficients Involving the 0perator's Measurement Capability

$\begin{array}{lr}\text { Variable } & \text { Coefficient } \\ \text { MCAP0P } & .00350 \\ \times 1 \times 4 & .00012 \\ \times 2 \times 4 & -.00013 \\ \times 3 \times 4 & .00162 \\ \times 4 \times 4 & -.02535 \\ \times 4 \times 5 & -.00575 \\ \times 4 \times 6 & .00400 \\ \times 4 \times 7 & .00113\end{array}$


TABLE 30. Coefficients Involving the Crossover Point, Gamma

\begin{tabular}{|c|c|}
\hline Variable & Coefficient \\
\hline GAMMA & .00021 \\
\hline$\times 7 \times 6$ & -.00037 \\
\hline$\times 2 \times 6$ & -.00025 \\
\hline$\times 3 \times 6$ & -.00100 \\
\hline$\times 4 \times 6$ & .00400 \\
\hline$\times 5 \times 6$ & -.00375 \\
\hline$\times 6 \times 6$ & -.05717 \\
\hline$\times 6 \times 7$ & .00137 \\
\hline
\end{tabular}

Conclusions Concerning Q $Q_{M A X}$. From the data presented thus far, the following conctusions can be drawn:

- The variables with the greatest impact on $Q_{\text {MAX }}$ are the inspector's measurement capability, the goal quantity, and the throughput.

- There are important squared terms and interactions. The presence of interactions means that the effect of a variable may depend on the level of some other variable. Any generalizations about the impacts of particular variables must take account of this possibility.

- There is still some lack of fit with a second order polynomial. The fit could be improved by inclusion of higher order terms. 
Total cost. The response variable called Total cost is defined as the total number of inspector man-days per year required in connection with routine inspections at a particular facility. It includes both off-site and on-site inspector activities. It is calculated as the sum of the inspection man-days required for Planning and Preparation, Audit Activities, Checking and Servicing Containment and Surveillance Devices, Post-inspection and Evaluation Activities, and Verification of Measurement Data. It should be noted that Total cost as defined here, does not correspond to either maximum routine inspection effort (MRIE) as defined in INFCIRC/153 or actual routine inspection effort (ARIE) as defined in facility Attachments. MRIE and ARIE include only man-days spent at the facility.

As was done with the response variable $Q_{\text {MAX }}$, many graphs were examined to note indications of relationships between Total cost and the input variables.

Figure 18 is a histogram of the values of Total cost for the 62 cases included in the experimental design. The values range from 130 to 500 mandays. The mean value is $\sim 265$. As was the case for $Q_{\text {MAX }}$, the data seem to fall into several groups, but the separation between the groups is less clear for Total Cost.

Figure ig is a plot of Total cost versus annual plant throughput; Figure 20 shows histograms of Total cost for each of the three levels of throughput. A strong relationship is evident--large throughput piants tend to require large inspection efforts. Plant throughput appeared to have the largest impact of any of the input variables. Another input variable which had a fairly strong effect was detection goal quantity. Figure 21 shows a plot of Total cost versus goal quantity (GQ); Figure 22 shows histograms of Total cost for the three levels of goal quantity. A fairly strong relationship is evident. The larger the goal quantity, the lower the total inspection cost. The mean value of Total cost decreases from 359 man-days to 223 man-days as the goal quantity increases from $25 \mathrm{~kg}{ }^{235} \mathrm{U}$ to $125 \mathrm{~kg}$ $235 \mathrm{U}$, approximately a $40 \%$ change in Tota? cost. The other plots of Total Cost against input variables are shown in Appendix $C$. 


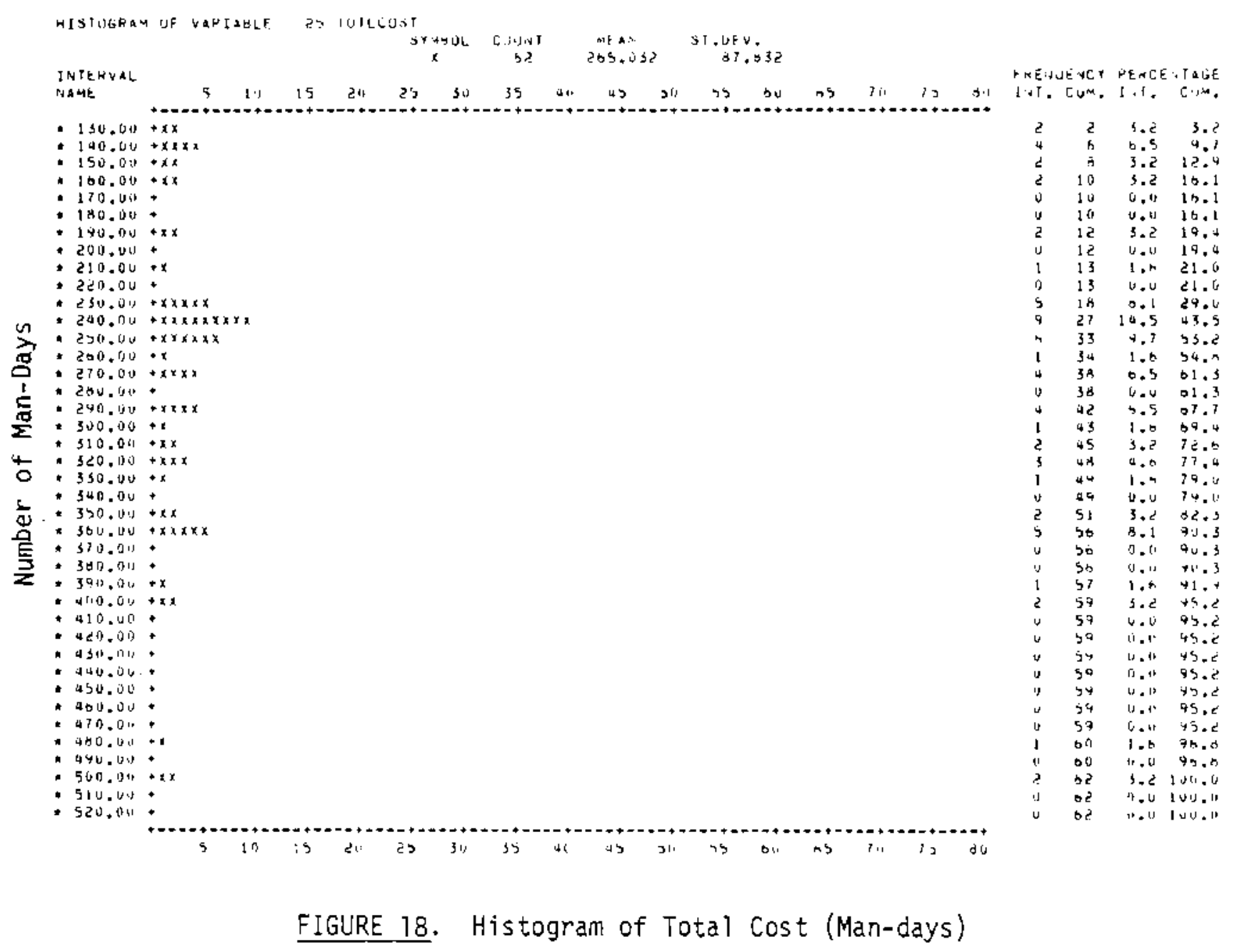




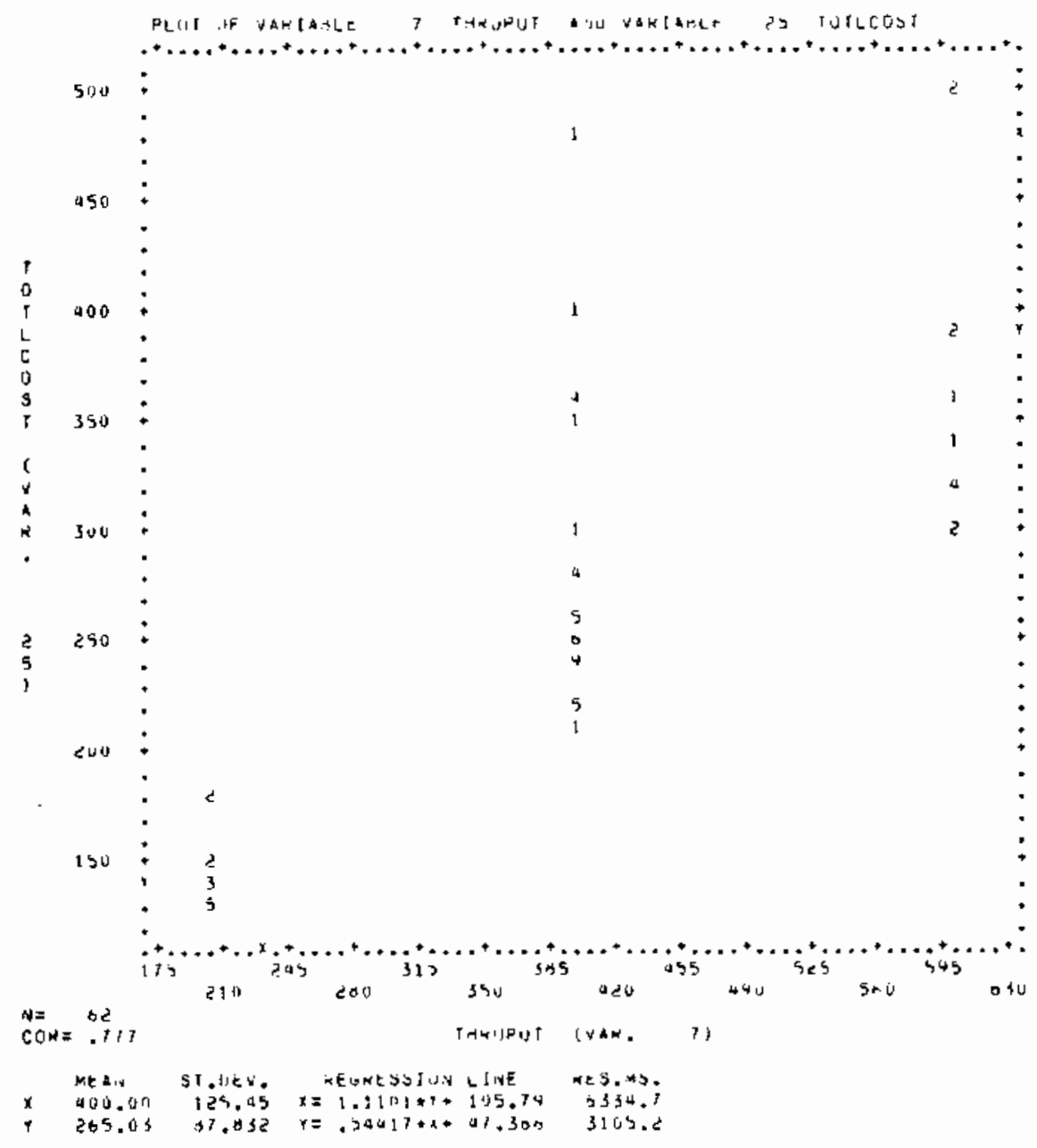

FIGURE 19. Total Cost Versus Throughput 


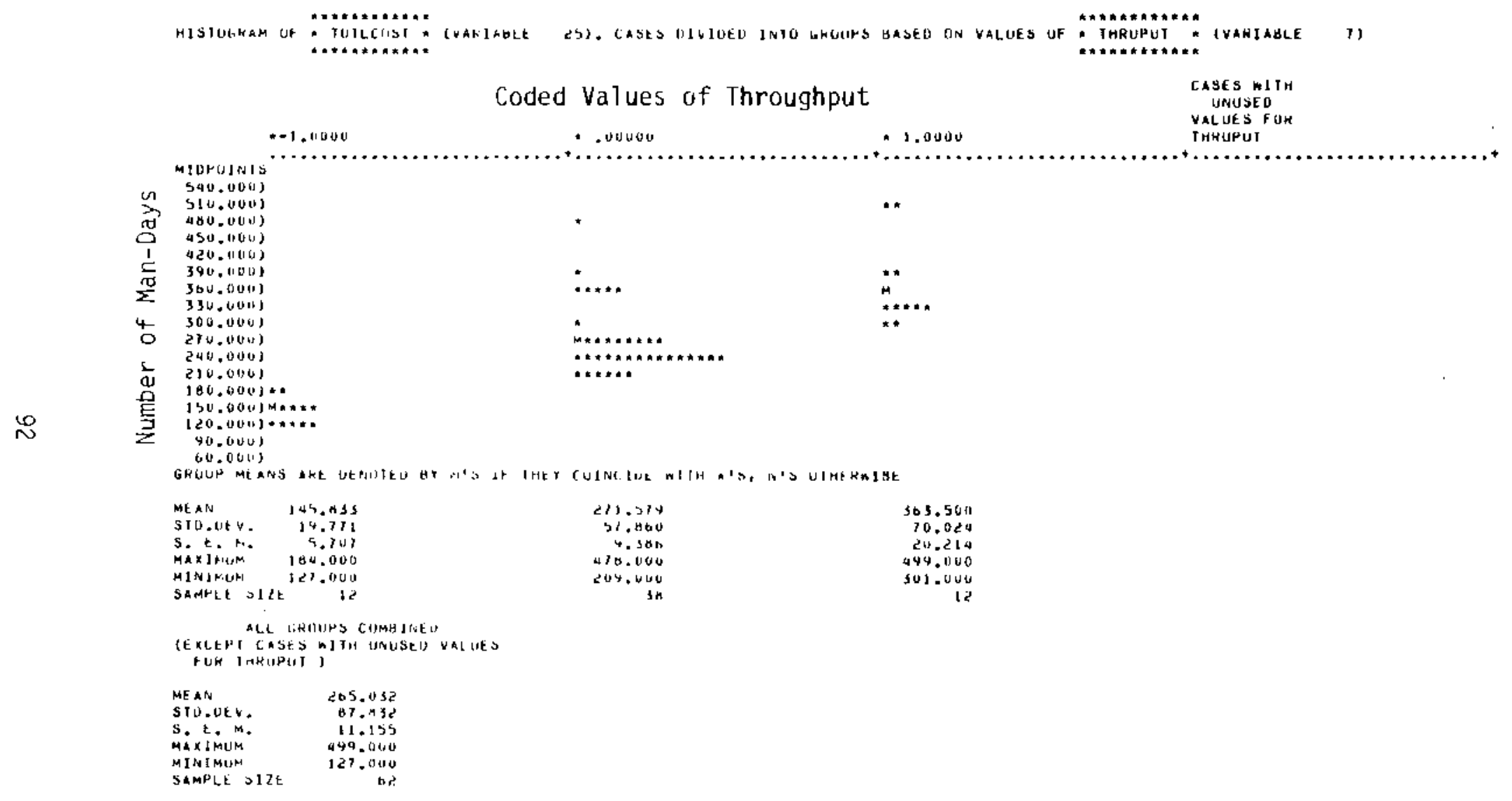

FIGURE 20. Histograms of Total Cost Versus Throughput 


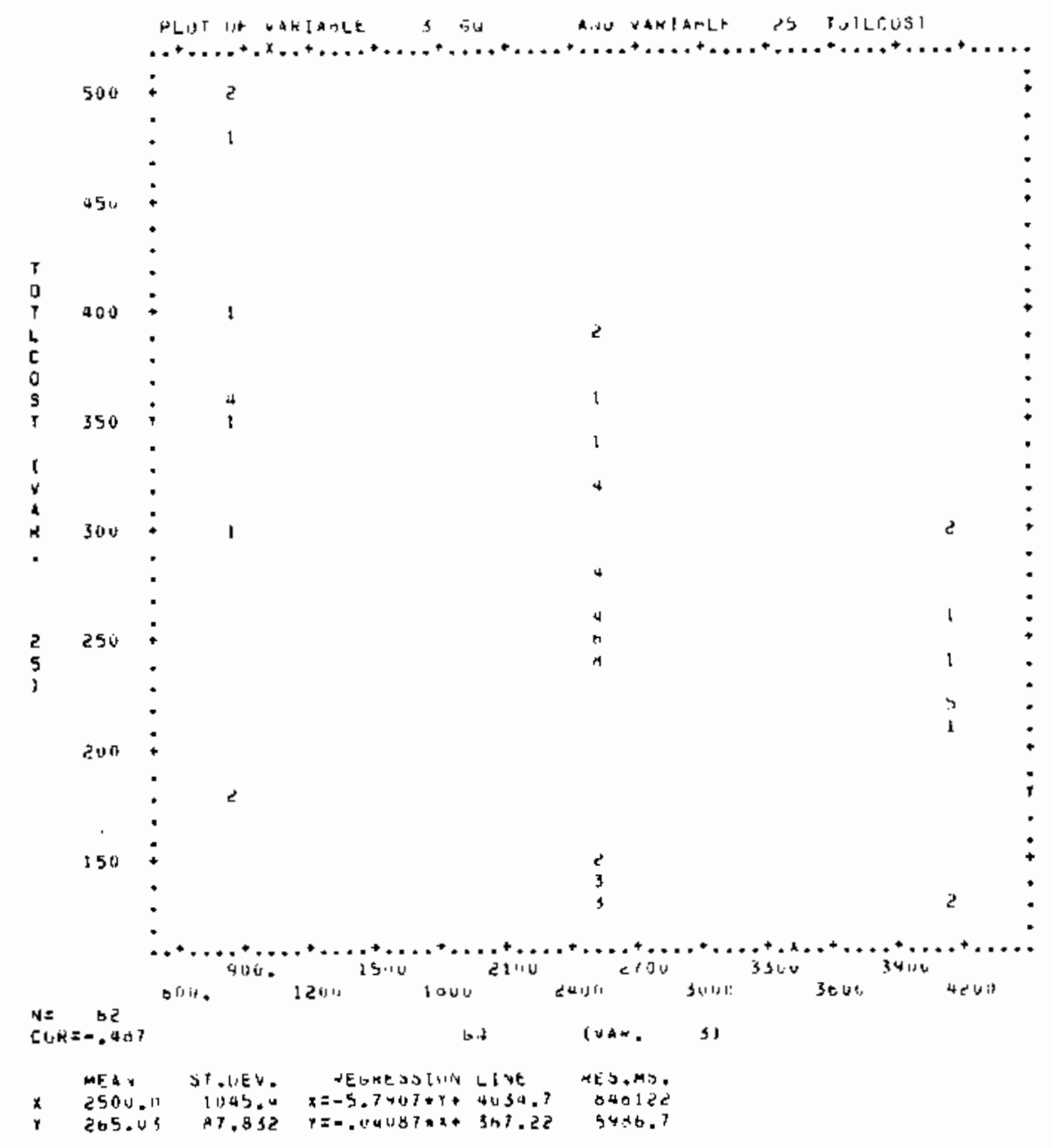

FIGURE 21. Total cost Versus Goal Quantity (GQ) 


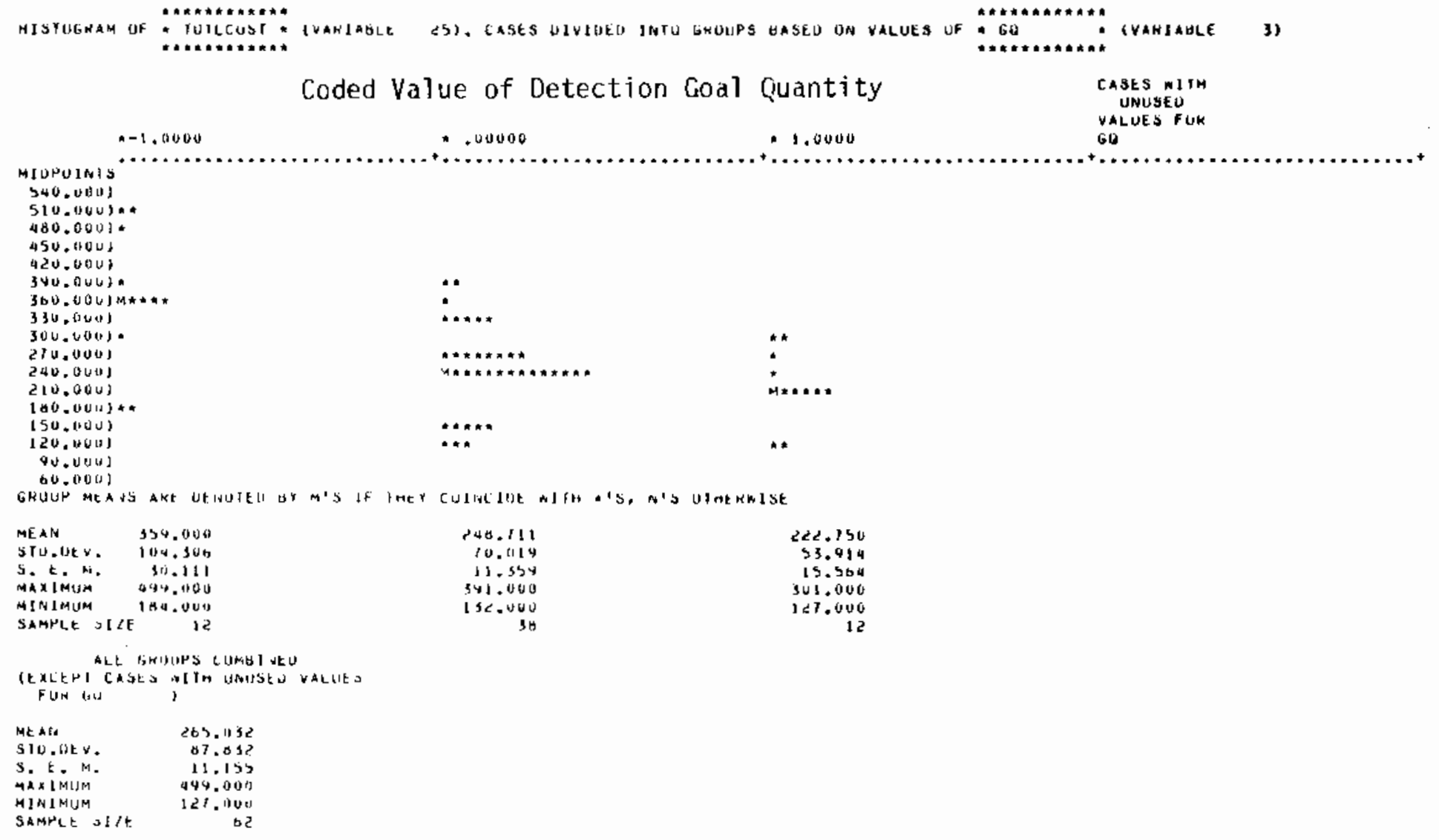

FIGURE 22. Histograms of Total Cost Versus Goal Quantity (GQ) 
Table 31 is a summary of the regression analysis of Total cost as a function of all the input variabies. The "MULTIPLE R-SQUARE" is about .99, which indicates that $99 \%$ of the variation in Total cost is explained or accounted for by the regression equation. The fit is better than for $0_{\text {MAX }}$, where the coefficient was .97 .

It is readily seen that the throughput is the most important variable. Table 32 shows the variables with regression coefficients larger than 10 in absolute value. The variables are ranked according to the absolute values of the coefficients. The table tends to indicate which variables have the greatest impact on Total cost.

As with $Q_{\text {MAX }}$, quadratic terms (e.g., $\times 3 \times 3$, or goal quantity squared) and interaction terms (e.g., $\times 3 \times 7$, or goal quantity times throughput) are important. Looking back at Figure 22 which showed the relationship between goal quantity and Total cost, one can in fact see some curvature, which shows up in the regression analysis as a large coefficient for $\times 3 \times 3$, or godl quantity squared.

The magnitude of the $x 3 \times 7$ interaction term means that the effect of goal quantity depends on the value of the throughput--it has a bigger impact when the throughput is large. Conversely, throughiput has a bigger impact when the goal quantity is large. The reason for this is related to the nature of attribute sampling. The variation in Total Cost associated with changes in goal quantity is driven primarily by the need for more attributes tests when the goal quantity is sma11. As the goal quantity increases, the fraction of the population that must be sampled decreases. The change in the fraction is the same, regard less of the population size, but the charge in the absolute number of items will obviously be greater when the population is large.

Conclusions Concerning Total Cost. The following conclusions concerning Total cost can be drawn:

- The variables with the greatest impact on Total cost are the throughput, the goal quantity and the goal probability of nondetection. 
TABLE 31. Summary of Regression Analysis: Total Cost

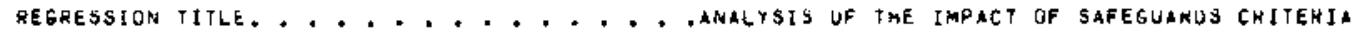

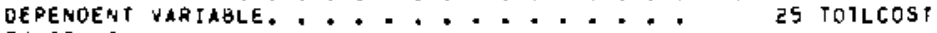

TLLERANCE LLL OATA CONSIOERED AS A SINGLE GRUUJP

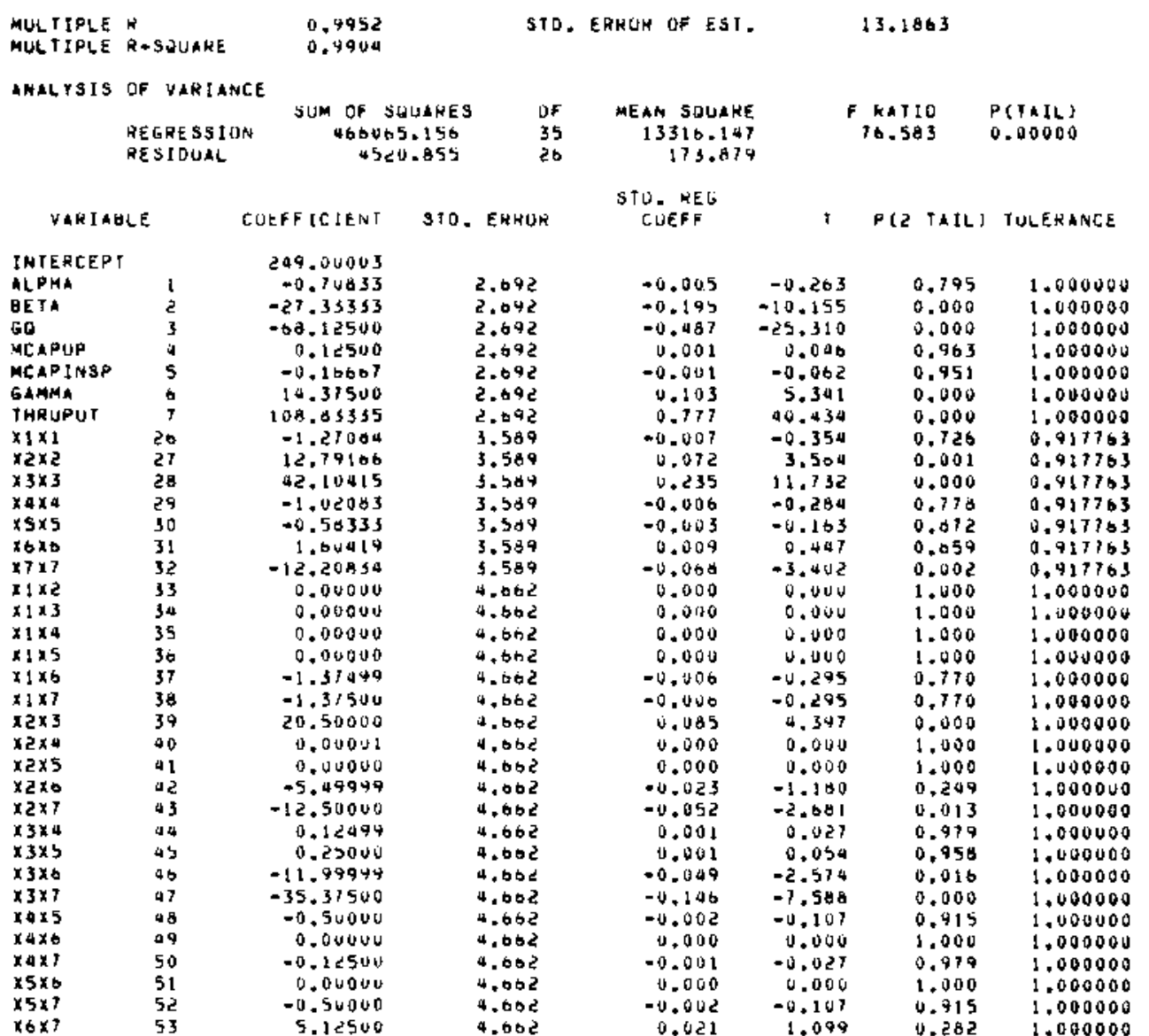


- There are important squared terms and interactions.

- The fit is quite good. Examination of the residuals shows that the largest percentage error of prediction is 14\%. Most of the errors are considerably smaller.

TABLE 32. Variables that Have the Most Impact on Total Cost

$\underline{\text { Variable }}$

THRUPUT

GQ

$\times 3 \times 3$

$\times 3 \times 7$

BETA

$\times 2 \times 3$

GAMMA

$\times 2 \times 2$

$x 2 \times 7$

$x 7 \times 7$

$\times 3 \times 6$

\section{Coefficient}

109

$-68$

42

$-35$

$-27$

21

14

13

$-13$

$-12$

$-12$ 


\section{DISCUSSION OF THE IMPACT OF THE INPUT VARIABLES}

In this section the role of each of the seven parameters considered in this study is discussed and conclusions concerning their effects on probability of detection and inspection costs are presented.

Role of Aipha

The role of the false alarm probability, alpha, in the overall safeguards decision structure has been the subject of some controversy recently. In fact, alpha has a number of distinct roles to play, corresponding to different levels of the decision structure.

In a strictly formal sense, alpha is the probability of rejecting the null hypothesis when it is in fact true; in more descriptive language it is the "false alarm" probability. The concept can be applied in various ways in the safeguards context, depending on how one defines the null hypothesis and what specific decisions and actions are contemplated when one speaks of rejecting the null hypothesis. At one extreme one can consider the null hypothesis to be something like: "There has been no diversion of safeguarded nuclear material to nuclear weapons or other nuclear explosive device," (paragraph 19, INFCIRC/153). Rejection of the null hypothes is in this case could be interpreted as a decision by the Board of Governors to report to all members of the IAEA and to the Security Council and General Assembly of the United Nations. Many other definitions of the null hypothes is are conceivable, and a variety of actions and decisions associated with rejection of that hypothesis are conceivable.

At the other extreme, one can consider a situation in which an inspector is attempting to verify a particular stratum of nuclear materiai on the basis of a set of verification measurements. In this case the null hypothe. sis might be that the operator's declared quantities of material are unbiased estimates of the true quantities. Rejection of the nutr hypothesis could be interpreted as a decision to perform some supplemental evaluation activity. 
Between the two extremes a great many possible nuil hypotheses, decisions, and actions can be defined. Sequences of hypotheses and decisions can also be considered, so that the decision by the Board of Governors to report to all the members of the IAEA and to the United Nations can be analyzed in terms of sequences of prior decisions leading eventually to a final decision by the Board.

Criteria for choosing values of alpha may be different, depending on what particular rull hypothesis is being considered and what particular actions and decisions ensue when the null hypothesis is rejected.

In this report we have focused our attention on null hypotheses concerning diversion of safeguarded nuclear material from a single low-enriched uranium conversion and fuel fabrication facility over a one-year period. We assume that when the null hypothesis is rejected then an investigation of possible causes is initiated. We have not considered in any detail exactly what activities that investigation might comprise. We considered a range of values for alpha $(\alpha=.01$ to $\alpha=.1)$ consistent with the recommendations of varjous working groups and panels.

Over the range of values considered in this study, alpha had only a minor impact on inspection costs or probability of detection. Increasing the false alarm probability led to a slight decrease in the probability of detection, as one would expect from theoretical considerations. But the range of values was narrow and the achieved probability of detection was in many cases constrained by timitations resulting from systematic measurement errors. Alpha would have a more pronounced impact on detection probability if a) detection capability were not constrained by systematic errors, and b) the range of values of alpha were extended. Figure 15 already gives some indication of what would happen if alpha were set at values smalier than .01: the detection probability seems to be decreasing more rapidly as the lower values of alpha are approached.

The effect of alpha on inspection costs was very slight. Again, this conclusion depends for its validity on the narrow range of alpha values studied, and on the fact that the inspection plan was constrained because of dominant systematic errors. In other circumstances, theoreticai considerations 
suggest that increasing alpha would tend to lead to a decrease in inspection costs. The effect could never be very great in a relative sense, though, because there are substantial components of inspection costs that are completely insensitive to changes in alpha.

\section{Role of Beta}

Beta is the goal probability of nondetection. The word goal is important here because, as shown in this and other studies, the goal probability of nondetection is not always achievable.

Formally, beta is the probability of accepting the null hypothesis when it is false. As with alpha, it is important to define clearly the hypotheses under consideration and to specify what decisions and actions are associated with acceptance or rejection of the null hypothesis.

In this report the null hypothesis is as stated earlier, the alternative hypothesis is that diversion of a goal quantity of safeguarded nuclear material has occurred from a given facility over a one-year period. We have considered a range of values of beta that is consistent with the recommendations of various advisory groups and panels.

Over the range of values considered in this study, and given the assumptions about plant size and measurement systems, beta had only a slight effect on the achieved probability of detection (1-Q $\left.Q_{M A X}\right)$. The effect on inspection costs was more noticeable, but was still fairly moderate.

The impact on probability of detection is somewhat surprising inasmuch as it means that the goal probability of detection has little bearing on the achieved probability of detection. The explanation for this result is that for many of the 62 cases the combined effects of plant size and systematic measurement errors precluded the attainment of the detection goal - the variances of the pertinent test statistics were too large. For such cases the achieved probability of detection is governed by the limiting systematic errors and is largely insensitive to variations in the (unattainable) goal probability. If, by virtue of small plant size and/or very good measurements, the detection goals were attainable, then the achieved probability of detection and the goal probability would be closely correlated. 
It also should be emphasized that we considered only a narrow range of values for beta. The impact of beta would be larger if the range were broadened. Nevertheless, Figure 13 shows that even if the goal probability of detection were as $10 \mathrm{w}$ as, 5ay, $20 \%$, there would still be cases where the goal probability was unattainable.

The effect of beta on inspection costs is oniy moderate. When beta increases from .01 to .1 , i.e., when the goal probability of detection decreases from $99 \%$ to $90 \%$, a reduction in inspection costs on the order of $15 \%$ to $20 \%$ is seen. (For a definition of the measure of inspection costs used in this report, see Appendix A.) Most of the change is due to reductions in the amount of attributes testing required to detect large defects. Again, there are substantial components of the cost of inspections that are insensitive to changes in beta, but as long as the cost of performing verification measurements is significant compared to all other costs, an increase in beta will lead to a noticeable reduction in costs.

Role of Goal Quantity

The goal quantity is defined as the quantity of material that, if diverted, is to be detected with a specified probability (given by $1-\beta$ ). In this report we are concerned with diversion from a given facility over a one-year period. We have considered a range of goal quantities from $25 \mathrm{~kg}$ $235 \mathrm{U}$ (833 kg uranium at $3 \%$ nominal enrichment) to $125 \mathrm{~kg} 235 \mathrm{U}(4166 \mathrm{~kg}$ uranium at $3 \%$ nominal enrichment). The midpoint of this range, $75 \mathrm{~kg}^{235} \mathrm{~J}$, has been agreed upon as the quantity of safeguards significance for uranium of enrichment less than $20 \%$.

Over the range of values considered, and given the assumptions used in this study, goal quantity had an appreciable effect on both probability of detection and inspection costs.

As the goal quantity increases, the probability of detection tends to increase. The explanation is clear: the larger the amount diverted the easier it is to detect, in general. The effect of goal quantity depends rather strongly on the throughput and on the inspector's measurement capability. Goal quantity has a smalier effect on detection probability when 
the throughput is large and when the inspector's measurement capability is weak. Actually, this result depends very much for its validity on the particular assumptions used in this report. The key factor to keep in mind is the relationship between detection capability, which is a function of throughput and the inspector's measurement capability, and goal quantity. When the detection capability is much worse than the goal quantity, as it is in many of the cases in this study, then the detection probability will be low and a small change in goal quantity will have little effect on the detection probability. Conversely, when the detection capability is much better than the goal quantity, the detection probability will be high and again a small change in goal quantity will have little effect. In the intermediate case, where the detection capability and the goal quantity are comparable in magnitude, a small change in goal quantity may have a relatively large impact on the detection probability. What we are seeing in this study is a lot of cases where the detection capability is considerably worse than the goal quantity (particularly when throughput is high or the inspector's measurement capability is weak) and a smaller number of cases where the detection capability and the goal quantity are comparable in magnitude.

The total cost of inspections is heavily influenced by the goal quantity: the smaller the goal quantity, the higher the cost. The diversion of a smalier amount requires more effort for detection. The effect is nonlinear, becoming more pronounced as the goal quantity gets smaller. There is also a large interaction with throughput: the effect tends to be greater when the throughput is large. As mentioned earlier, this behavior is associated with the nature of attributes testing, where the fraction sampled, rather than the absolute number sampled, plays a crucial role.

Role of Operator's Measurement Capability

In this study the term "operator's measurement capability" refers to the standard deviations associated with various measurement operations carried out by the plant operator. If these standard deviations are small, then the operator's measurement capability is good.

There are many other characteristics of the operator's accounting systeri, and more generally of the State's System of Accounting and Control, that have 
an impact on the costs and effectiveness of IAEA safeguards; for example, the timeliness of reporting, procedures and practices for physical inventory taking, the availability of up-to-date, stratified inventory listings, and the organization of storages. Although such factors are important, they are not included in our sensitivity analysis. Therefore, our conclusions about the effect of the "operator's measurement capability" must be interpreted with caution; we are only talking about measurement error standard deviations and we are implicitly assuming that good accounting and control practices are followed in any event.

Given the assumptions used in this study, and for the ranges of values considered here, the operator's measurement capability had very little impact on the probability of detection. The mathematical reason for this somewhat counter-intuitive result is the following: the probability of detection is calculated on the basis of the MUF- $\hat{D}$ statistic and the variance of MUF-D does not depend on the operator's systematic errors. Only the random errors of the operator enter into the MUF-D statistic and they tend to be completely dominated in the variance of MUF- $\hat{D}$ by the inspector's systematic errors (see pages 33-34 of BNWL-1852 for the mathematical details). Furthermore, it should be noted that the probability of detection, as we use the term here, refers to the probability of detecting the diverter's best strategy. If one were to consider another index of effectiveness, such as the probability of detecting diversion assuming no falsification (in other words, diversion into MUF), then the operator's measurement errors could have more impact.

The operator's measurement capability aiso had a minimal effect on inspection costs. Again, in interpreting this finding, the qualifications mentioned above should be borne in mind.

Role of Inspector's Measurement Capability

The term "inspector's measurement capability" as used here refers to the standard deviations of the inspector's variables measurement methods. The inspector's attributes measurement methods are discussed in terms of the crossover point, ganma. 
Given the assumptions used in this study, and over the range of values considered, the inspector's measurement capability was the variable with the greatest impact on the probability of detection. Its effect on inspection costs, however, was minimal.

Figure 17 showed very clearly the strong effect on QMAX. The effect is highly nonlinear and there is an appreciable interaction with goal quantity. A smalier interaction with throughput can also be noted. The explanation for these findings is that the inspector's measurement error variances, together with material quantities, are the primary determinants of the variance of MUF- $\hat{D}$. The relation between the goal quantity and the standard deviation of MUF- $\hat{D}$, in turn, is a determining factor in calculating the probability of detection.

The inspector's measurement capability has little effect on costs because only one relatively minor component of inspection costs, namely, the costs associated with variables sampling, depends on the measurement capability. Moreover, in many cases variables sampling is limited by diminishing returns considerations -- beyond a certain point the variance of MUF- $\hat{D}$ and the probability of detection are relatively insensitive to increases in variables sample sizes so the sampling plans are sometimes truncated (see pages 24-25 of ISPO-58 by Wincek and Mullen; also Part III of ISPO-35, Estimation of Inspection Effort).

Role of Gamma, the Crossover Point

The crossover point, garma, is a measure of the detection sensitivity of the inspector's attributes testing instruments. Typical1y, gamma is chosen to be four to six times the relative standard deviation of a single attributes measurement. Operationally, what this means is that if a diverter were to falsify an item, creating a discrepancy of gamina times the item weight, such a falsification could be detected with essential1y 100\% probability and $0 \%$ chance of a false alarm provided the item were subjected to an attributes test.

In this study we found that gama had no significant impact on the probability of detection and a slight but noticeable impact on inspection 
costs. Improvements in the sensitivity of the attributes tests tended to lead to small (less than 10\%) savings in inspection costs.

Role of Throughput

In this study the plant throughput was varied from 200 to 600 tons of uranium per year. Actually, the throughput is only a partial measure of the size and complexity of a plant's operation-- inventory, plant layout, process design, and many other characteristics of the facility have an impact on safeguards effectiveness and efficiency-- but it is the only plant parameter, other than measurement system parameters, that we varied in this study. Calculations, as well as theoretical considerations, show that throughput is likely to be the key plant-specific parameter for determining inspection costs and effectiveness in low-enriched uranium conversion and fuel fabrication plants.

For the range of values considered in this study, the throughput had a major impact on the probability of detection. The tendency was not unexpected: the larger the throughput, the lower the probability of detection. The effect was highly nonlinear. There was an important interaction with goal quantity (as discussed eariier). The explanation for the drop in detection probability as throughput increases is simply that the detection goals become increasingly difficult to attain as the throughput (and hence the variance of MUF- $\hat{D}$ ) becomes large in relation to the goal quantity.

The effect of throughput on inspection costs is even stronger. Figure 20 showed the trend quite clearly. The effect is slightly nonlinear. There is, as mentioned earlier, an important interaction between throughput and goal quantity and a smaller interaction between throughput and beta. 


\section{CONCLUSIONS}

The extensive exercising of the mathematical model in the programs of INSPECT gave rise to several conclusions:

- For the low enriched uranium conversion and fuel fabrication facility outlined in this study, the goal probability of detection of diversion was seldom achieved. Of the seven input variables which were systematically varied, the inspector's measurement capability had the greatest impact on the probability of detection. Detection goal quantity and plant material throughput also showed strong impacts.

- The total cost of inspections in man-days as defined in this study was heavily influenced by the size of the goal quantity with a large interaction with plant throughput. Somewhat surprising was the lack of influence of the inspector's measurement capability. This was discussed in the previous section.

One other important feature of the study was the development of reasonably good prediction equations for achievable detection probabilities $\left(1-Q_{\text {MAX }}\right)$ and inspection costs. These equations are shown in tabular form as Tables 23 through 32. The equations are valid over the range of the input variables studied as shown in Table 19. These equations allow for "quick and dirty" calculations to be performed for facilities where complete design information is not available. Similar equations can be developed for other responses using the data provided in Table 21. 


\section{REFERENCES}

1. IAEA Safeguards Technical Manual, IAEA-174, Part F: Statistical Concepts and Techniques, International Atomic Energy Agency, Vienna, 1977.

2. BMDP-77, Biomedical Computer Programs, P-Series, W.J. Dixon, Series Editor, M.B. Brown, Editor 1977 Edition, University of California Press, 1977. For this study, PDP-11 Version 2.0, Revised July 1978, was used.

3. G.E.P. Box, The Exploration and Exploitation of Response Surfaces: Some General Considerations and Examples, Biometrics, March 1954, pp. 16-60.

G.E.P. 80x and K.B. Wilson, On the Experimental Attainment of Optimum Conditions, Journal of the Royal Statistical Society, Series B, Vol. 13, No. $1,1951, \mathrm{pp} .1-45$.

W.J. Hill and W.G. Hunter, A Review of Response Surface Methodology: A Literature Survey, Technometrics, Vol. 8, No. 4, November 1966, pp. 571-590.

W.G. Cochran and G.M. Cox, Experimental Designs, 2nd Edition, John Wiley and Sons, 1957.

R.H. Myers, Response Surface Methodology, Allyn and Bacon, Boston, 1971.

4. G.E.P. Box and D.W. Behnken, Some New Three Level Designs for the Study of Quantitative Variables, Technometrics, Vol. 2, No. 4, November 1960, pp. $455-475$.

5. G. Hough, T. Shea, and D. Tolchenkov, Technical Criteria for the Application of IAEA Safeguards, in Proceedings of a Symposium on Nuclear Materia] Safeguards, IAEA-SM-231/11 2 , Vienna, 1979.

6. "The Structure and Content of Agreements Between the Agency and States Required in Connection with the Treaty on the Nonproliferation of Nuclear Weapons," INFCIRC/153 (corrected), IAEA, Vienna, 1972.

7. M.A. Wincek and M.F. Mullen, INSPECT - A Package of Computer ProgramS for Planning Safeguards Inspections, PNL-2559, ISP0-58, Pacific Northwest Laboratory, Richiand, Washington, 1979.

8. Strategy of Experimentation, Applied Technology Division, E. I. du Pont de Nemours \& Co., Wilmington, Delaware, 1975.

9. G.E.P. Box and J.S. Hunter, Multi-Factor Experimental Designs for Exploring Response Surfaces, Annals of Matheriatical Statistics, Vol. 28, 1957, pp. 195-241.

10. R.A. Schneider, et al., Example of Verification and Acceptance of Operator Data - Low Enriched Uranium Fabrication, BNWL-1852, Pacific Northwest Laboratory, Richland, Washington, August 1974. 
APPENOIX A

ESTIMATION OF INSPECTION EFFORT

\section{INTRODUCTION}

The objective of IAEA safeguards is "the timely detection of diversion of significant quantities of nuclear material from peaceful nuclear activities," and "the technical conclusion of the Agency's verification shall be a statement in respect of each material balance area' (MBA) of the amount of material unaccounted for (MUF) over a specific period, giving the limits of accuracy of the amounts stated." [Reference INFCIRC/153] To fulfill this objective, and according to safeguards agreements, the Agency has the right to perform different types of inspections for independent collection of information. These inspections include ad hoc inspections, special inspections, and routine inspections. This appendix concerns itself only with the routine inspections.

Routine inspections constitute a major portion of all inspection activities. Some of the major tasks which may constitute an inspection are as follows:

1) Verification of the consistency of information contained in records and reports,

2) Verification of nuclear material subject to safeguards,

3) Other verification activities.

There are many inspection activities required to fulfill these tasks. These include for flow verification: (Ref: IAEA/STR-79)

- Examination of records, verification of self-consistency and consistency with reports, updating book inventory, filling in all documents for samples, preparing samples for shipment;

- Application, examination, removal, and renewal of seals;

- Servicing and review of surveiliance equipment; 
- Selection of items to be sampled and observation of sampling;

- Item identification, counting and measurement of rods and assemblies (weighing and NDA);

- Observation of the calibration and carrying out of calibration for a 71 measurement equipment;

- Activities at the rod loading station (sampling, NDA);

- Verification of the quality of operator's measurement system including analytical and NDA equipment using independent standards.

Some of the major activities for inventory verification include:

- The above flow verification activities;

- Verification of the operator's physical inventory taking for completeness and accuracy;

- Weighing of containers with nuclear material on the basis of random sampling plan;

- Taking accountability samples;

- Identification and counting of fuel assemblies and rods and the use of NDA techniques for their verification;

- Containment/surveitlance activities.

To make the estimation of inspection effort somewhat easier, the above tasks are compressed into five major categories:

- Planning and preparation (including followup actions from previous inspections)

- Audit (includes records audit, comparison between records and reports, recording of book inventory, verification of quality and functioning of operator's measurement system and activities in respect to MUF's, SRD's, and accidental losses)

- Check and service containment and surveillance devices

- Post-inspection and evaluation 
- Verification of measurement data (includes verification of inventory changes and/or material flow and verification of inventory).

PLANNING AND PREPARATION

Planning and preparation includes such activities as review of reports, preparation of the material balance, calculation of the sampling plan, and travel to the facility. The amount of effort required for these activities is expected to depend on the following factors:

- The number and kind of inspections to be performed in the one-year period

- The size of the plant

- Travel time to the plant

- The effectiveness of the SSAC

- Prior experiences of the IAEA at this specific facility or similar facilities.

In this study, planning and preparation effort is estimated as follows:

Inspection effort for planning and preparation = the number of inspections for flow verification per year, times the effort required per flow verification, plus the number of inspections for inventory verification year, times the effort required per inventory verification.

Plant size enters as a factor as more inspections are needed in the larger plants, and more flow and inventory inspections are also undertaken. Table A.l summarizes the numbers used in this study.

TABLE A.1 Routine Inspection Effort for One-Year Period Planning and Preparation

\begin{tabular}{|c|c|c|c|c|c|}
\hline $\begin{array}{c}\text { Plant Size } \\
\text { (tons throughput) }\end{array}$ & $\begin{array}{l}\text { Man-Days } \\
\text { at Facility }\end{array}$ & $\begin{array}{l}\text { Man-Days } \\
\text { Travel } \\
\end{array}$ & $\begin{array}{l}\text { Man-Days } \\
\text { in Field }\end{array}$ & $\begin{array}{c}\text { Man-Days } \\
\text { at } H Q \\
\end{array}$ & $\begin{array}{c}\text { Total } \\
\text { Man-Days } \\
\end{array}$ \\
\hline 200 & 0 & 19 & 19 & 27 & 40 \\
\hline 400 & 0 & 28 & 28 & 34 & 62 \\
\hline 600 & 0 & 41 & 41 & 43 & 84 \\
\hline
\end{tabular}


It should be noted that man-days in the field is the sum of man-days of travel and man-days at the facility, and total man-days is the sum of man-days at $H Q$ and man-days in the field. The values were arrived at as follows:

Trave? Requirements for the 200 Ton Plant -

two inspectors, each one day of travel, for each of eight flow verifications, plus three inspectors, each one day of travel, for the one inventory verification.

$$
(2 \times 8)+(3 \times 1)=19 \text { man-days }
$$

Trave 1 Requirements for the 400 Ton Plant -

two inspectors, each one day of travel, for each of nine flow verifications, plus five inspectors, each one day of travel, for two inventory verifications.

$$
(2 \times 9)+(5 \times 2)=28 \text { man-days }
$$

Travel Requirements for the 600 Ton Plant -

three inspectors, each one day of travel, for each of nine flow verifications, plus seven inspectors, each one day of travel, for two inventory verifications.

$$
(3 \times 9)+(7 \times 2)=41 \text { man-days }
$$

Headquarters Effort for the 200 Ton Plant -

two man-days per flow verification, eight verifications per year, plus five man-days for the inventory verification.

$$
(2 \times 8)+(5 \times 1)=21 \text { man-days }
$$

Headquarters Effort for the 400 Ton Plant -

two man-days per flow verification, nine verifications per year, plus eight man-days for each inventory verification, two per year.

$$
(2 \times 9)+(8 \times 2)=34 \text { man-days per year }
$$

Headquarters Effort for the 600 Ton Plant -

three man-days per flow verification, nine verifications per year, plus eight man-days for each inventory verification, two per year.

$$
(3 \times 9)+(8 \times 2)=43 \text { man-days per year }
$$


AUDIT

Audit includes inspection activities such as records examination, and a check for compliance with established procedures. The amount of effort required for these activities is expected to depend on the following factors:

- The number and kind of inspections to be performed

- Size of the plant

- The effectiveness of the SSAC.

In this study, audit inspection effort is established as follows:

Inspection effort for audit = the number of flow verifications per year, times the inspection effort per flow verification, plus the number of inventory verifications per year, times the effort per inventory verification.

Table A.2 summarizes the numbers used in this study.

IABLE A.2 Routine Inspection Effort for One-Year Period

\begin{tabular}{ccccccc}
$\begin{array}{c}\text { Plant Size } \\
\text { (tons throughput) }\end{array}$ & $\begin{array}{c}\text { Man-Days } \\
\text { at Facility }\end{array}$ & $\begin{array}{c}\text { Man-Days } \\
\text { Trave] }\end{array}$ & $\begin{array}{c}\text { Man-Days } \\
\text { in Field }\end{array}$ & $\begin{array}{c}\text { Man-Days } \\
\text { at H0 }\end{array}$ & $\begin{array}{c}\text { Total } \\
\text { Man-Days }\end{array}$ \\
\cline { 1 - 2 } 200 & 21 & 0 & 21 & 0 & 21 \\
400 & 43 & 0 & 43 & 0 & 43 \\
600 & 54 & 0 & 54 & 0 & 54
\end{tabular}

These values were obtained as follows:

Inspection Effort for Audit Activities - 200 Ton Plant -

two man-days per flow verification, eight verifications per year, plus five man-days per inventory verification, one verification per year.

$$
(2 \times 8)+(5 \times 1)=21 \text { man-days }
$$

Inspection Effort for Audit Activities - 400 Ton Plant -

three man-days per flow verification, nine verifications per year, 
plus eight man-days per inventory verification, two verifications per year.

$$
(3 \times 9)+(8 \times 2)=43 \text { man-days }
$$

Inspection Effort for Audit Activities - 600 Ton Plant -

four man-days per flow verification, nine flow verifications per year, plus 11 man-days per inventory verification, two verifications per year.

$$
(4 \times 9)+(9 \times 2)=54 \text { man-days }
$$

\section{CHECK AND SERVICE CONTAINMENT AND SURVEILLANCE DEVICES}

The effort required for this inspection effort is small compared to the other major categories. For the 200 ton throughput plant, this value was assumed minimal. The other two plants were each allotted one man-day. There might be items under seal which would need to be checked.

\section{POST-INSPECTION AND EVALUATION}

The effort required for post-inspection and evaluation activities is expected to depend on the following factors:

- The amount of data collected in the course of inspection

- Travel time for returning to Headquarters

- The effectiveness of SSAC

- The number and kind of inspections

- Plant size

The following relationship is used to calculate this effort:

Inspection effort for post-inspection and evaluation = the number of flow verifications per year, times the effort required per flow verification, plus the number of inventory verifications, times the effort required per inventory verification.

Table A. 3 summarizes these results. 
TABLE A.3 Routine Inspection Effort for One-Year Period

\section{Post-Inspection and Evaluation}

\begin{tabular}{|c|c|c|c|c|c|}
\hline $\begin{array}{c}\text { Plant size } \\
\text { (tons throughput) }\end{array}$ & $\begin{array}{l}\text { Man-Days } \\
\text { at Facility }\end{array}$ & $\begin{array}{l}\text { Man-Days } \\
\text { Trave? } \\
\end{array}$ & $\begin{array}{l}\text { Man-Days } \\
\text { in Fiejd } \\
\end{array}$ & $\begin{array}{l}\text { Man-Days } \\
\text { at } \mathrm{HO} \\
\end{array}$ & $\begin{array}{c}\text { Total } \\
\text { Man-Days } \\
\end{array}$ \\
\hline 200 & 0 & 19 & 19 & 21 & 40 \\
\hline 400 & 0 & 28 & 28 & 36 & 64 \\
\hline 600 & 0 & 47 & 41 & 37 & 78 \\
\hline
\end{tabular}

Travel values are the same as those found in Table A.?. The Headquarters man-days were determined as follows:

Headquarters Effort for the 200 Ton Plant -

two man-days per flow verification, eight verifications per year, plus five man-days per inventory verification, one verification per year.

$$
(2 \times 8)+(5 \times 1)=21 \text { man-days }
$$

Headquarters Effort for the 400 Ton Plant -

2.5 man-days per flow verification, nine verifications per year, plus seven man-days per inventory verification, two verifications per year.

$$
(2.5 \times 9)+(7 \times 2) \cong 37 \text { man-days }
$$

Headquarters Effort for the 600 Ton Plant -

2.5 man-days per flow verification, nine verifications per year, plus seven man-days per inventory verification, two verifications per year.

$$
(2.5 \times 9)+(7 \times 2) \cong 37 \text { man-days }
$$

\section{VERIFICATION OF MEASUREMENT DATA}

The fifth component of the estimation of inspection effort is verification of measurement data. These inspection activities consist of an item check, the attributes plan, and the variables plan. Factors affecting the item check include: 
- The nature, size, and layout of the facility

- The quality of the SSAC

- The number and kind of inspections

Factors affecting the attributes and variables plan include:

- Detection goal quantities

- Probability of detection

- The nature, size, and layout of the plant

- The effectiveness of the SSAC

- The quality of the operator's measurement system

- The inspector's measurement capability.

The computer code package INSPECT was used to calculate the sampling plans. The inspection effort for measurement verification is estimated as follows:

Inspection effort for verification of measurement data $=$ attributes sample size, times the effort required per attributes sample, plus the variables sample size, times the effort required per variables sample, plus the amount of effort required for the item check.

To evaluate the effort required, it was assumed that 15 minutes of inspection effort per attributes sample, and one hour per variables sample are necessary. It is also assumed that the item check takes five man-days for the 200 ton plant, ten man-days for the 400 ton plant, and 15 man-days for the 600 ton plant. 
APPENDIX B

INVESTIGATION OF BOX-BEHNKEN DESIGN INPUT VARIABLES

Generally speaking, good designs tend to be "bold" in the sense that the experimental points are widely spread out over the region of interest, balanced and symmetrical, and the input variables are uncorrelated as much as possible. It is interesting to examine the Box-Behnken design from these points of view.

Figures B.I through B.17 are histograms of the values of input variables for the 62 cases included in the experimental design that are not already included in the main text. The data shown are coded.

Figures B.1 through B.6 are for the first order input variables. The pattern is the same for alt seven (i.e., balance and symetry) with 12 low values (cases), 12 high values, and 38 middle values.

Figures B.7 through B. 12 are for the squared terms. Again they all follow the same pattern, as they must because the first order terms did. Note that there are only two levels here instead of three as there were for the first order terms. This is because the low value $(-1)$ squared is the same as the high value (1) squared.

Figures $B .13$ through $B .17$ are for the cross-product or interaction terms involving alpha, the false alarm probability. All the histograms for interaction terms follow the same pattern with four cases at the low value, four at the high value, and the remaining 54 cases at the middle value.

Clearly, the Box-Behnken design has characteristics of balance and symmetry as far as the levels of the predictor variables are concerned.

To look at possible correlations between input variables, we can examine scatter plots of the variables, one versus another, and also calculate correlation coefficients between pairs of variables.

Figures B. 18 through B.22 are scatter plots of alpha, the false alarm probability, versus all of the other first order inputs. The same pattern is evident in all the plots. There are two cases in each of the "corners" 


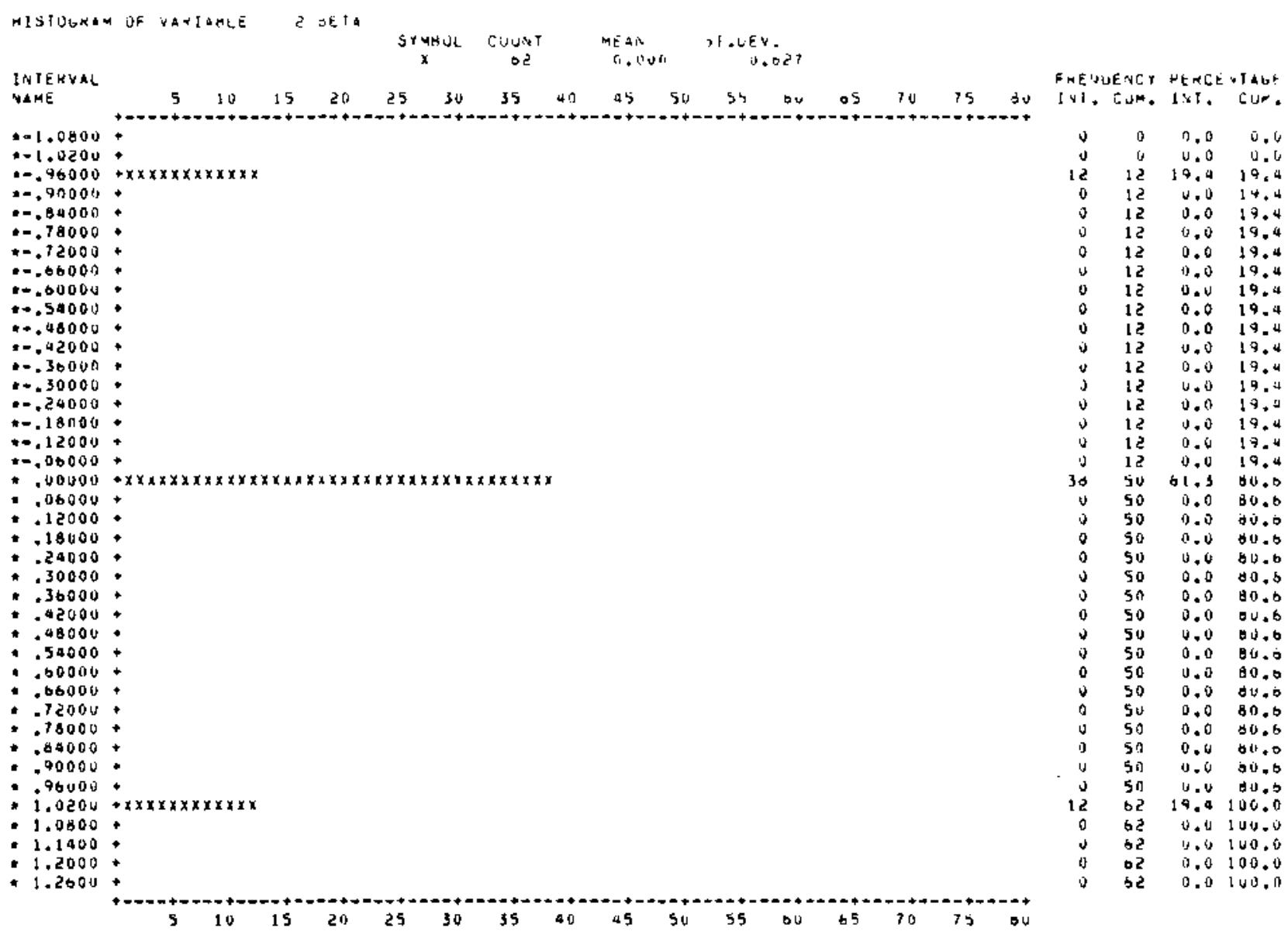

FIGURE B.l Histogram of Beta (Goal Probability of Non-detection) 


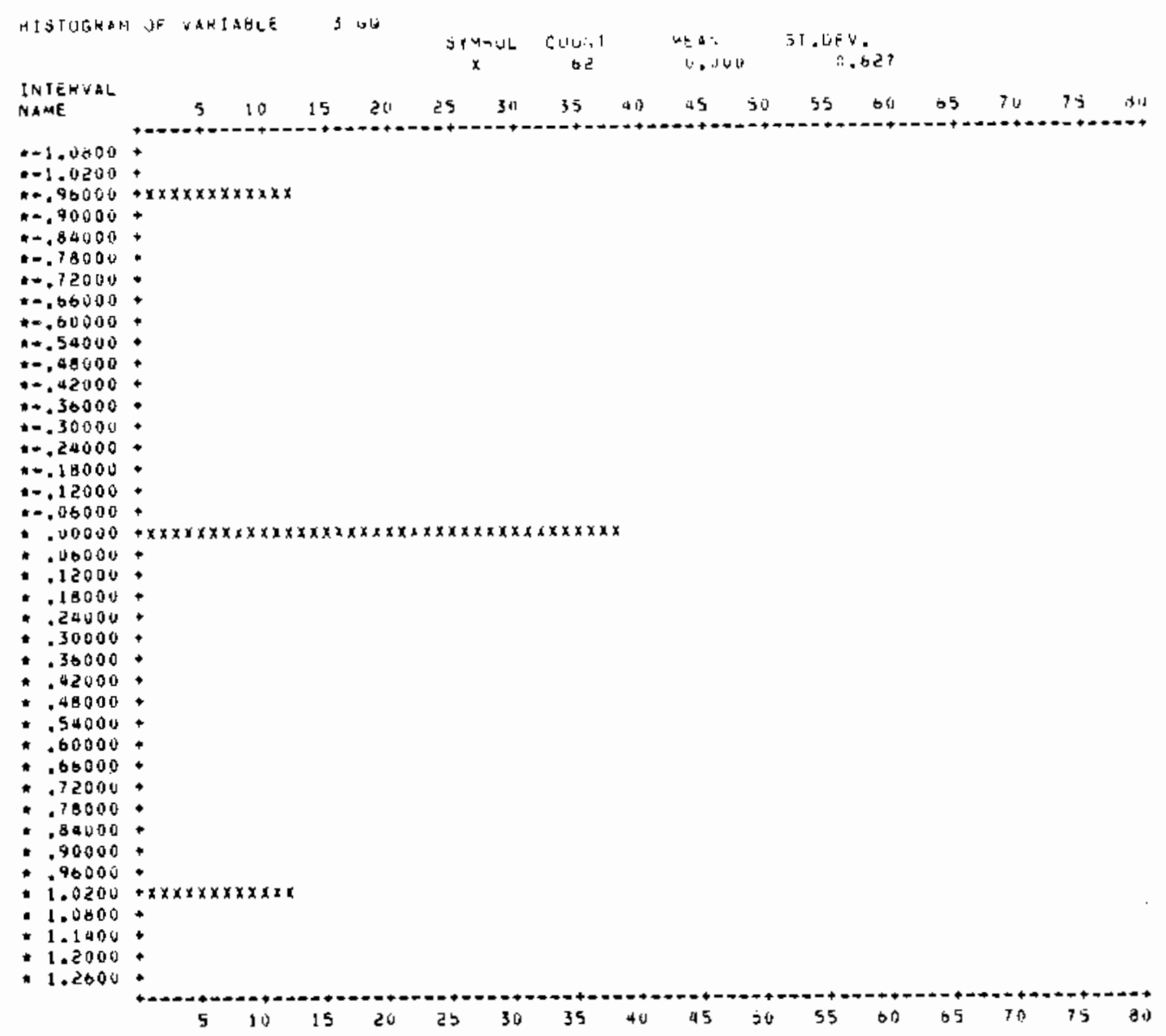

FremUENCY PERCE:UTAE

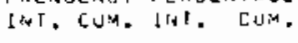

年

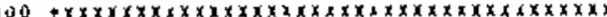

1.1400

1.2000

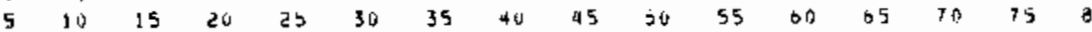

\begin{tabular}{|c|c|}
\hline 0.0 & $v, v$ \\
\hline 0.4 & 6.1 \\
\hline 19.4 & 14.4 \\
\hline$u . v$ & 14.4 \\
\hline U.v & 19.4 \\
\hline 0.0 & 17.4 \\
\hline 0.0 & $1+.0$ \\
\hline 0.0 & 19.4 \\
\hline 0.0 & 19.4 \\
\hline 0.0 & 19.4 \\
\hline 0.4 & 19.4 \\
\hline 0.0 & \\
\hline 0.0 & 19.4 \\
\hline 0.0 & 19.4 \\
\hline 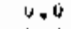 & 19.4 \\
\hline 0,0 & 14.4 \\
\hline 0.0 & 19.4 \\
\hline 0.0 & 19.4 \\
\hline 01,3 & $\forall 0, B$ \\
\hline 0.0 & 80.0 \\
\hline 0.0 & 80.0 \\
\hline$v+v$ & 80.0 \\
\hline 0.0 & $8 w=0$ \\
\hline 0.0 & 80.0 \\
\hline 0.0 & 80.0 \\
\hline 0.0 & 60.6 \\
\hline 0.0 & 80.0 \\
\hline U.0 & 30.6 \\
\hline 0.0 & 80.5 \\
\hline 0.0 & 80.6 \\
\hline 0.0 & 00,0 \\
\hline 0.0 & bी. 6 \\
\hline 0.0 & 80.0 \\
\hline 0.0 & 60.6 \\
\hline 8.0 & 00.0 \\
\hline 19.4 & 100.0 \\
\hline 0.0 & 100.0 \\
\hline 0.0 & 100.0 \\
\hline 0.0 & 100.0 \\
\hline 0.0 & 100.0 \\
\hline
\end{tabular}

FIGURE B.2 Histogram of GQ (Goal Quantity) 


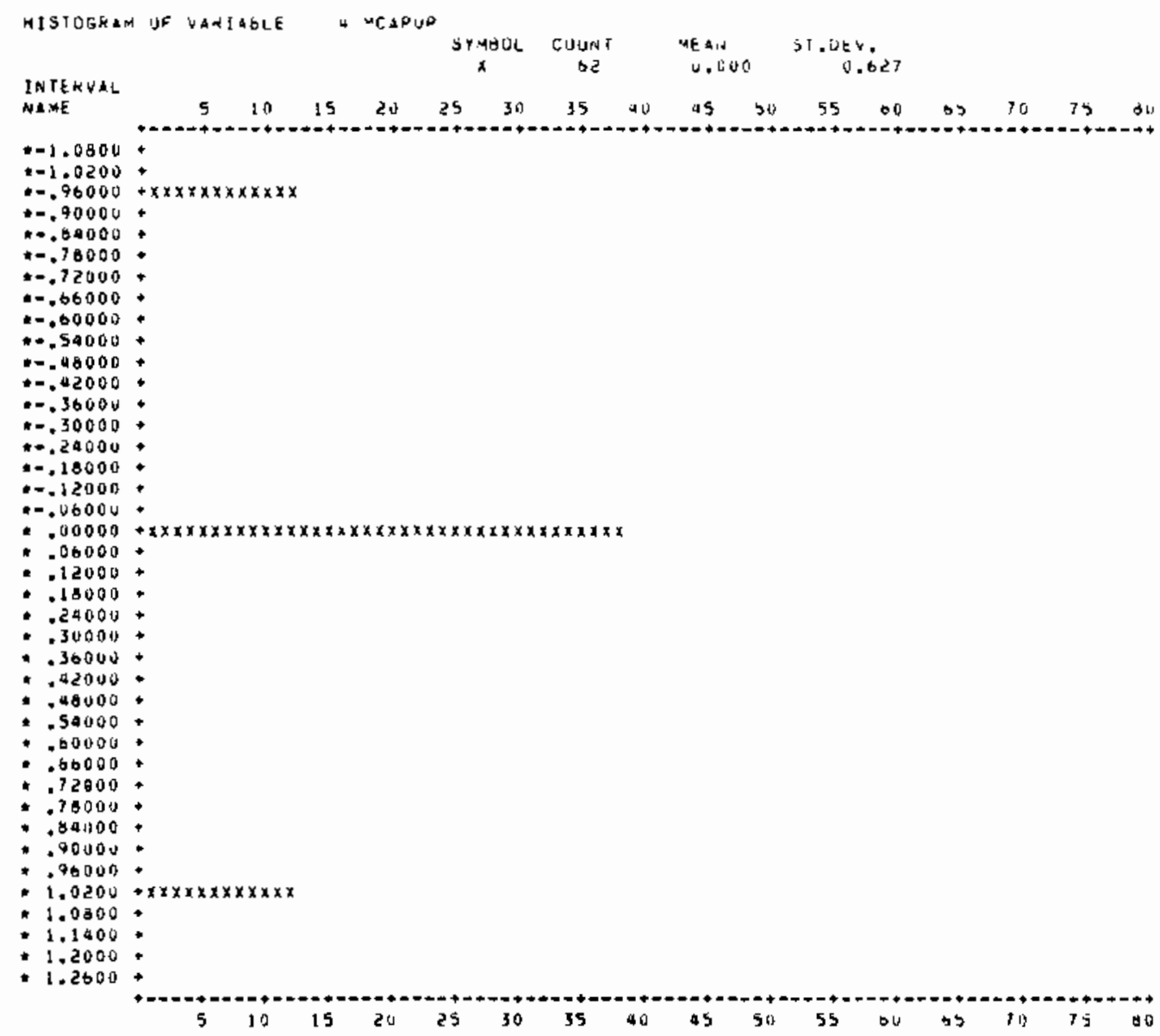

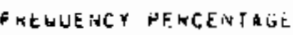
INT. CUM. Divt. CiJM.

\begin{tabular}{|c|c|c|c|}
\hline 0 & 0 & 0.0 & 50 \\
\hline 0 & is & 0.0 & 0.0 \\
\hline 12 & 12 & 19.4 & 19.4 \\
\hline 0 & 12 & 0.0 & 19.4 \\
\hline 0 & 12 & 0.0 & 19,4 \\
\hline v & 12 & 0.0 & 19.4 \\
\hline 0 & 12 & 0.0 & 14.4 \\
\hline 0 & 12 & 0.0 & $1 \% .4$ \\
\hline$v$ & 12 & 6.0 & 19.4 \\
\hline 0 & 12 & 0.0 & 19.4 \\
\hline 0 & 12 & 0.0 & 19.4 \\
\hline 0 & 12 & 0.0 & 14.4 \\
\hline$\checkmark$ & 12 & 0.0 & 19.4 \\
\hline$J$ & 12 & 0.0 & 19.4 \\
\hline 0 & 12 & 0.0 & 14.4 \\
\hline 0 & 12 & 0.0 & 19.4 \\
\hline 0 & 12 & 4.0 & 19.4 \\
\hline v) & 12 & 0.0 & 19.4 \\
\hline 56 & 50 & 1.3 & 80.6 \\
\hline$v$ & 50 & 0.0 & 00.15 \\
\hline a & 50 & 0.0 & 80.6 \\
\hline 0 & so & 0.0 & 00.6 \\
\hline 0 & 50 & 0.4 & 80.0 \\
\hline$v$ & Su & 0.0 & 40.0 \\
\hline$\dot{v}$ & 50 & 0.0 & $\forall 0+b$ \\
\hline 0 & 50 & 0.0 & 00.6 \\
\hline 0 & 50 & 0.0 & 80.6 \\
\hline 0 & 5o & 0.0 & 80.0 \\
\hline 0 & So & 0.0 & a 0,0 \\
\hline 0 & 50 & 0.0 & 50.0 \\
\hline v & 50 & 0.0 & 80.6 \\
\hline u & 50 & 0.0 & db.o \\
\hline v & 50 & $v .0$ & 80.0 \\
\hline v & bu & 4.0 & $a v .0$ \\
\hline 0 & 50 & 0.0 & $b$ \\
\hline 12 & bC & 19.4 & 100,0 \\
\hline 0 & 62 & 0.0 & 100.0 \\
\hline 0 & 62 & 0.0 & 100.0 \\
\hline 0 & 62 & 0.0 & 100.0 \\
\hline 0 & 62 & 0.10 & 160.0 \\
\hline
\end{tabular}

FIGURE B.3 Histogram of MCAPOP (Operator's Measurement Capability) 


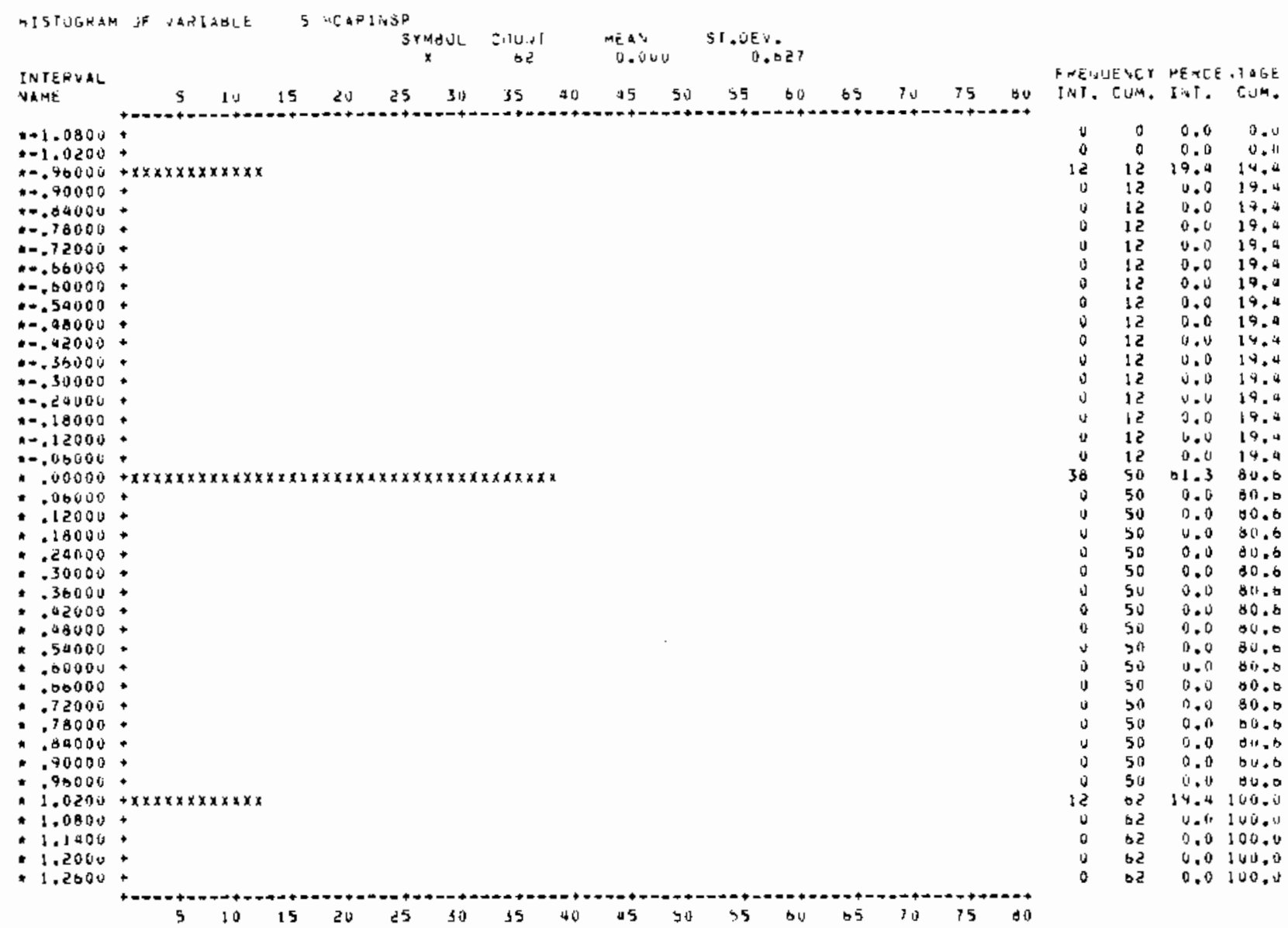

FIGURE B.4 Histogram of MCAPINSP (Inspector's Measurement Capability) 


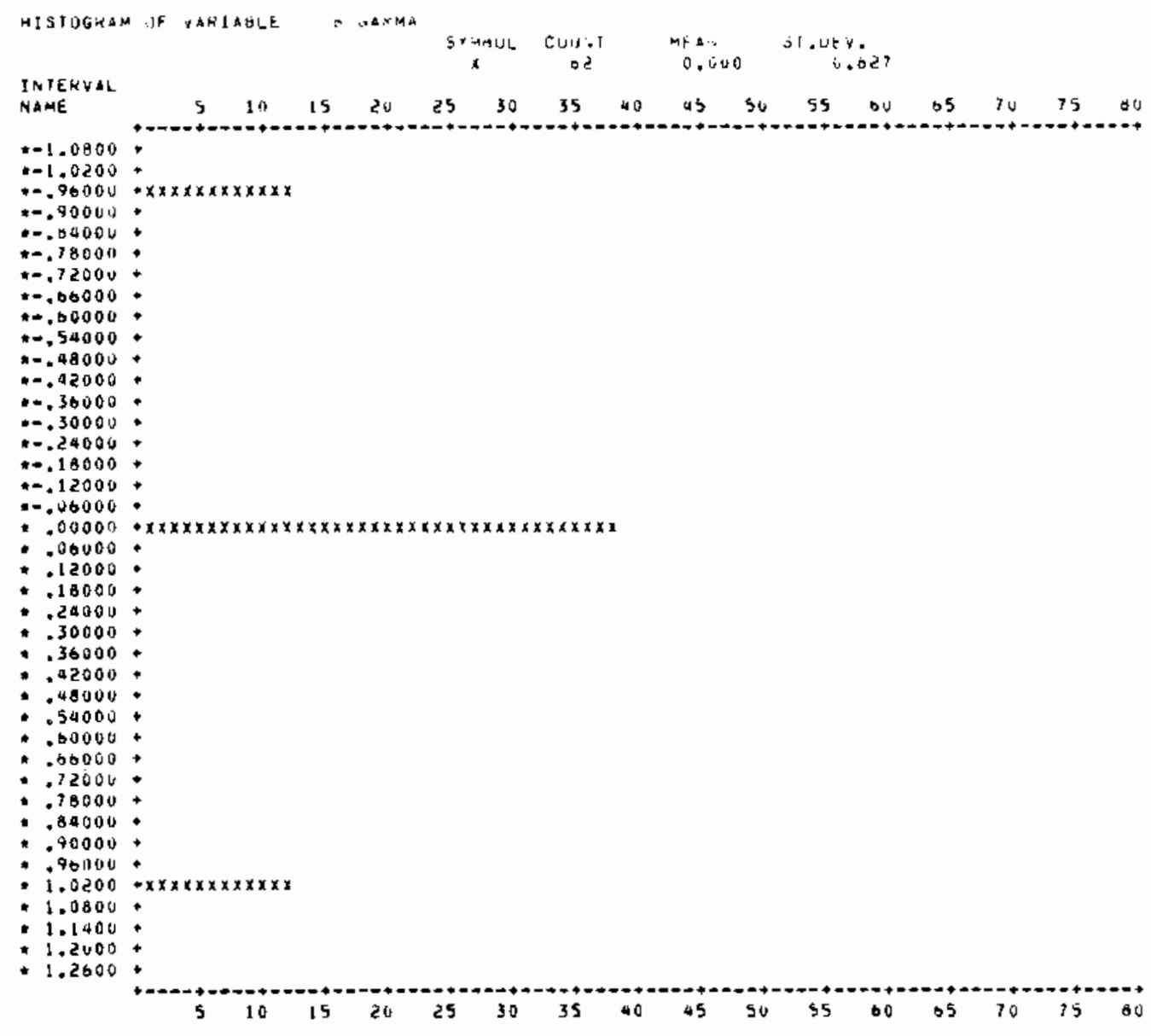

FHEDUENCY PEACEUTAGE INT. CUM, IVT, TUM.

\begin{tabular}{|c|c|c|c|}
\hline (3) & u & 1.0 & 0,0 \\
\hline 0 & 0 & 0.0 & 0.4 \\
\hline 12 & 12 & 19.4 & 19.4 \\
\hline D & 12 & 0.0 & $1+0.0$ \\
\hline 0 & 12 & 0.0 & 17.4 \\
\hline 0 & 12 & 0.0 & 18.4 \\
\hline 0 & 12 & 0.0 & 19.4 \\
\hline 0 & 12 & 0.0 & 19.4 \\
\hline 0 & 12 & 0.0 & 19.4 \\
\hline 0 & 12 & 0.0 & 19.4 \\
\hline 0 & 12 & 0.0 & .4 \\
\hline 0 & 12 & 0.0 & .4 \\
\hline 0 & 12 & 0.0 & 19.4 \\
\hline 0 & 12 & 0.0 & 9.9 \\
\hline 0 & 12 & 0.1 & 14,4 \\
\hline 0 & 12 & 4.0 & 19.4 \\
\hline v & 12 & 0.0 & 19.4 \\
\hline$v$ & 12 & U.u & 14.4 \\
\hline 36 & 50 & 01.3 & 80.0 \\
\hline$u$ & 50 & 0.0 & a1). b \\
\hline$\checkmark$ & so & 0.0 & 80.6 \\
\hline 0 & so & 10.0 & 80.0 \\
\hline 0 & 50 & 0.0 & 80.6 \\
\hline 0 & 50 & 0.0 & 60.0 \\
\hline$\dot{v}$ & 50 & 0.0 & $\Delta u, b$ \\
\hline 0 & 50 & 0.0 & 80.6 \\
\hline 0 & 50 & 0.0 & su.t \\
\hline$v$ & 5i) & 0.0 & $\$ 0.6$ \\
\hline j & 50 & 4.0 & 80.5 \\
\hline 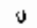 & 50) & 0.0 & 00.0 \\
\hline$v$ & So & 0.0 & 0.0 \\
\hline 0 & 50 & 0.0 & 00.6 \\
\hline 0 & 50 & 0.0 & $80, B$ \\
\hline 0 & So & 0.0 & du.t \\
\hline 0 & 30 & 0.0 & 80.6 \\
\hline 12 & b2 & 19.4 & 100.0 \\
\hline 0 & 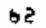 & 0.0 & 100.0 \\
\hline 0 & 02 & 0.0 & 100,0 \\
\hline 0 & 62 & 0.0 & 100.0 \\
\hline J & bz & 0.0 & 100.0 \\
\hline
\end{tabular}

FIGURE B.5 His togram of Gamma (Crossover Point) 


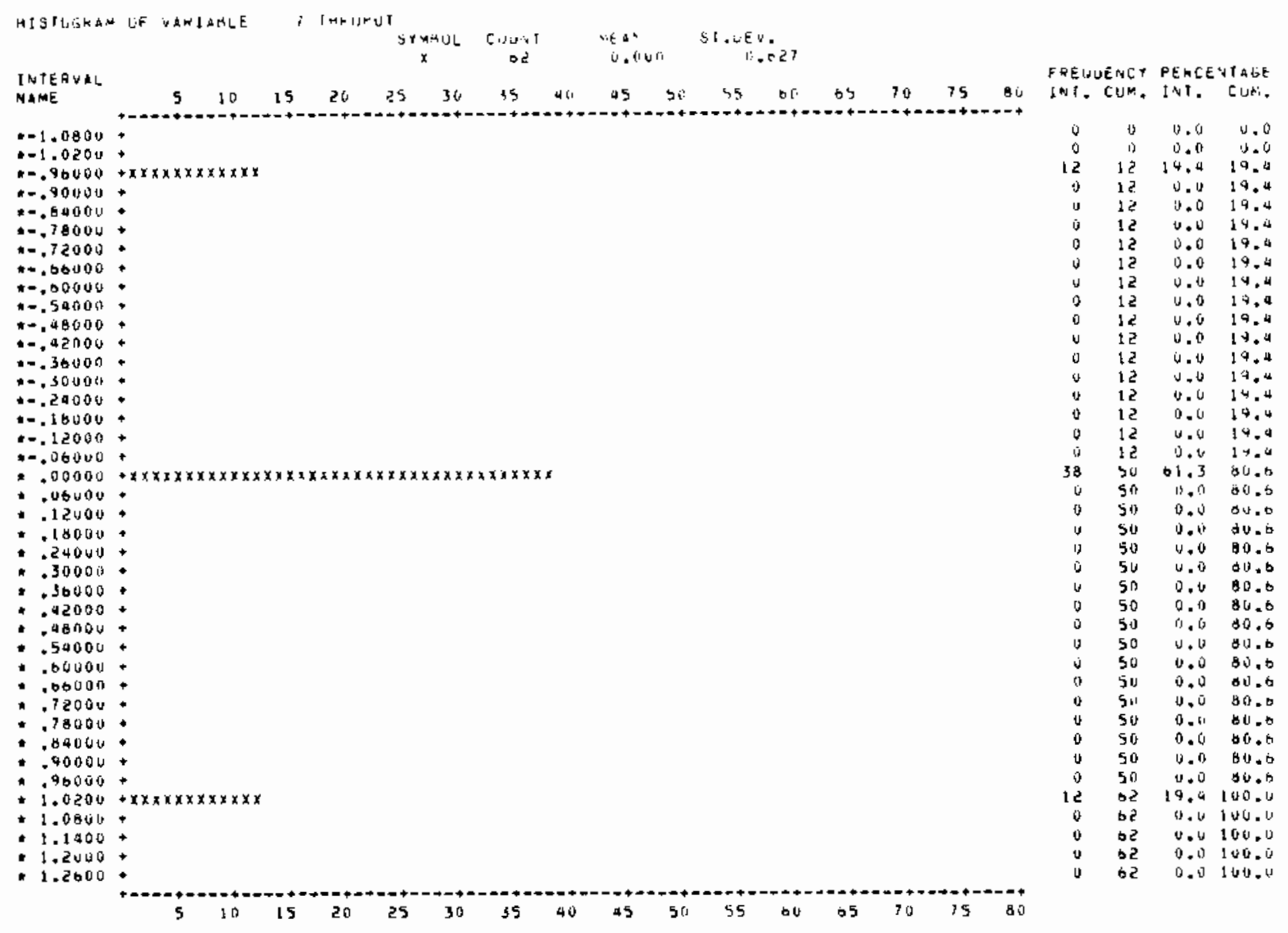

\section{FIGURE B.6 Histogram of Throughput}




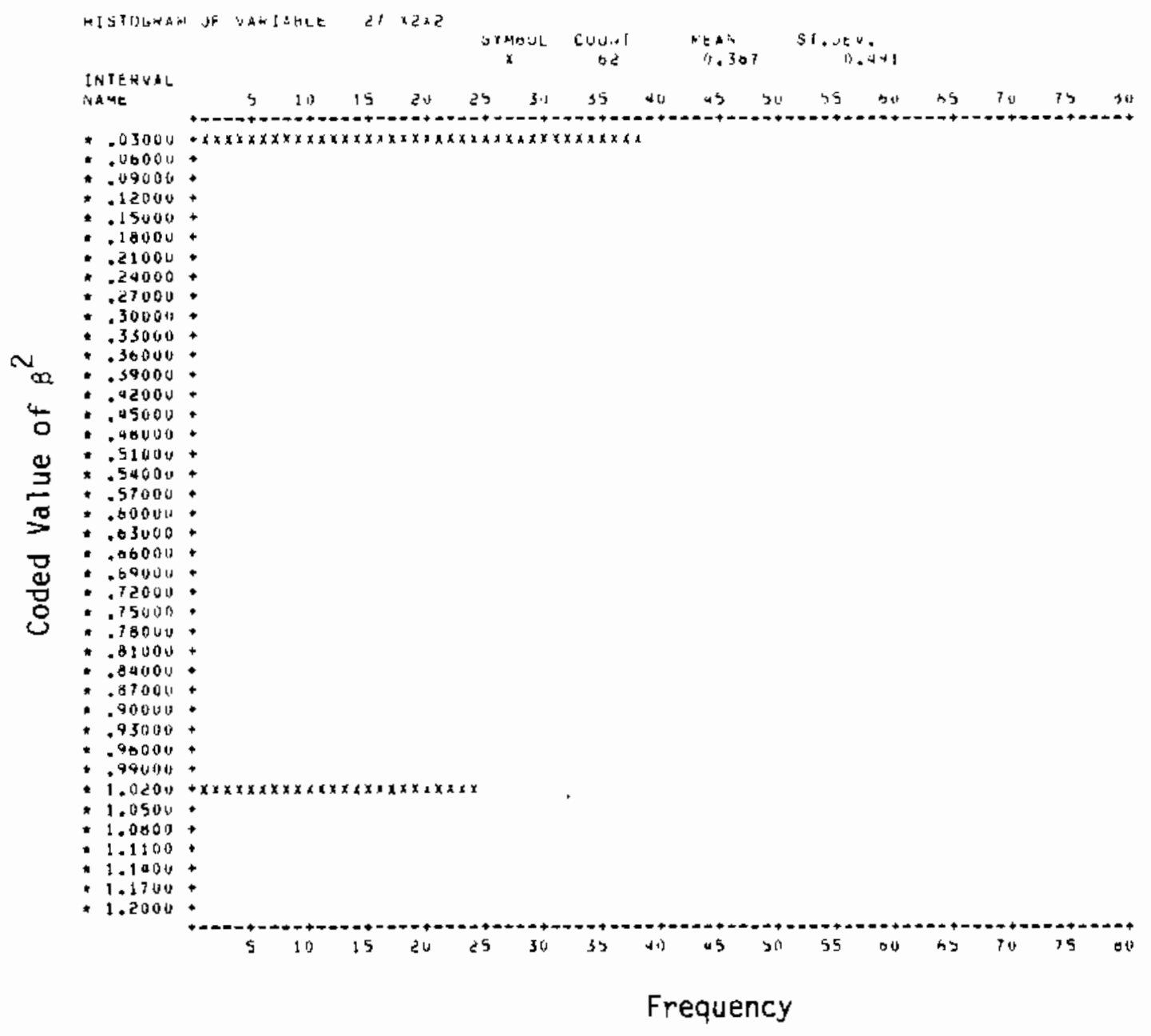

Eatidue oct rekrentate

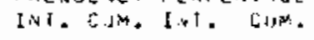

\begin{tabular}{|c|c|c|c|}
\hline 56 & $3 n$ & 61.3 & 01.3 \\
\hline$v$ & $3 n$ & v. & 01.3 \\
\hline$v$ & Só & w. & $\$ 1.3$ \\
\hline 0 & 38 & 0.6 & $=1.3$ \\
\hline 1) & 30 & 4.4 & 01.3 \\
\hline 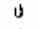 & 38 & U. 11 & b 1.3 \\
\hline$v$ & 36 & $u, 0$ & b 1.3 \\
\hline 0 & 36 & 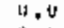 & 01.3 \\
\hline 0 & 38 & $a .0$ & $6: .3$ \\
\hline 4 & 30 & $u, 4$ & 61.3 \\
\hline$u$ & $3 \mathrm{H}$ & 4.0 & 01.3 \\
\hline$\psi$ & 34 & is.u & a). 3 \\
\hline 1) & 36 & 4.0 & 61.3 \\
\hline 0 & 38 & v. & 01.3 \\
\hline 4 & 38 & 0.0 & b. 1.3 \\
\hline is & 38 & U. 0 & 01.3 \\
\hline$v$ & 34 & U. & 01.3 \\
\hline$u$ & 34 & 0.3 & 61.3 \\
\hline$v$ & 38 & 12.4 & 1.3 \\
\hline$v$ & 58 & i. 6 & 01.3 \\
\hline$v$ & $3 t$ & 4.6 & 61.3 \\
\hline$v$ & 30 & $v .0$ & 01.3 \\
\hline$u$ & 30 & 0.0 & 01.3 \\
\hline U & $3 n$ & 4.6 & 01.5 \\
\hline i) & 30 & $6=0$ & 01.5 \\
\hline$\checkmark$ & 38 & $i .0$ & 61.3 \\
\hline$D$ & 39 & 4.0 & 01.3 \\
\hline v & 33 & 4.6 & 61.5 \\
\hline v & 36 & U. L & $=1.3$ \\
\hline 4 & 38 & 0.0 & 01.3 \\
\hline 0 & 38 & 0.0 & 01.3 \\
\hline u & $3 \mathrm{~A}$ & v. & 01.3 \\
\hline ' & 38 & 4.5 & 61.3 \\
\hline 24 & 02 & 30.7 & 100.0 \\
\hline$v$ & 02 & v. 0 & 140.0 \\
\hline$v$ & प्र & $u .4$ & 100.0 \\
\hline 1) & $b 2^{2}$ & $u \cdot v$ & IvU."1 \\
\hline 0 & 62 & 0.0 & 160.2 \\
\hline$v$ & 32 & u. o & 100.0 \\
\hline$v$ & $6 ?$ & 13.0 & 100.0 \\
\hline
\end{tabular}

\section{FIGURE B.7 Histogram of $\times 2 \times 2$ (Beta Squared)}




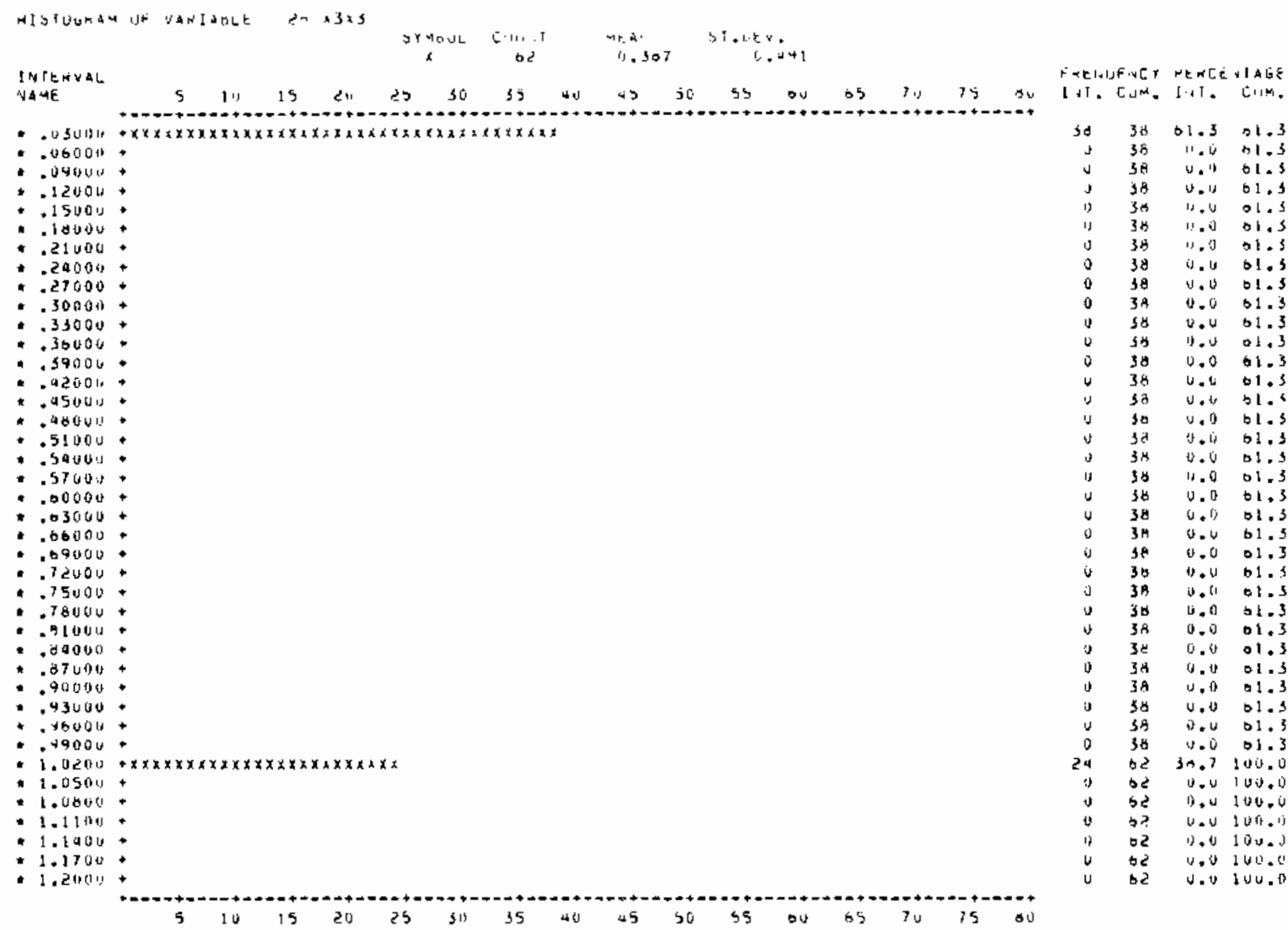

FIGURE 3.8 Histogram of $\times 3 \times 3$ (Goal Quantity Squared) 


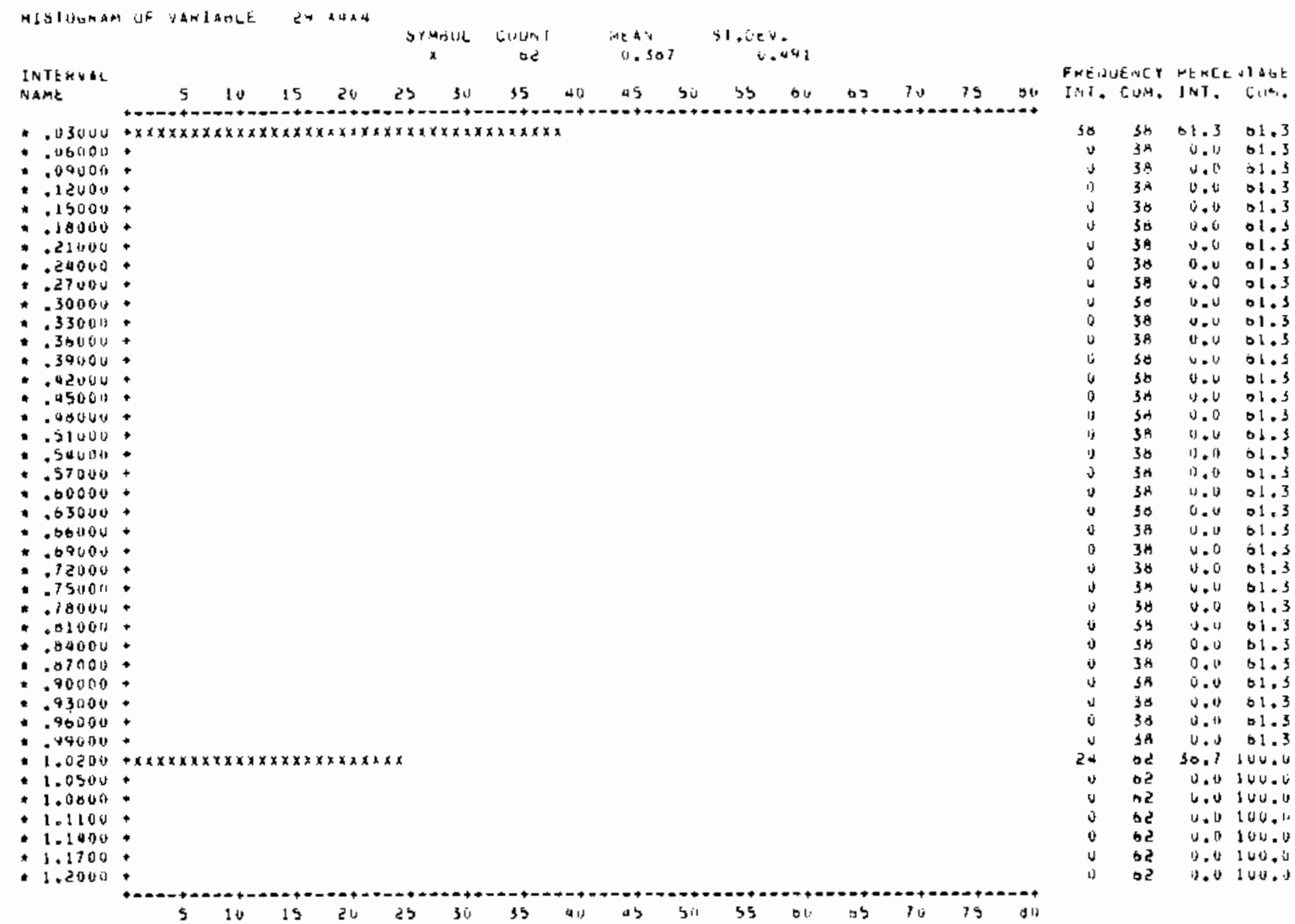

\section{FIGURE B.9 Histogram of $\times 4 \times 4$ (MCAPOP Squared)}




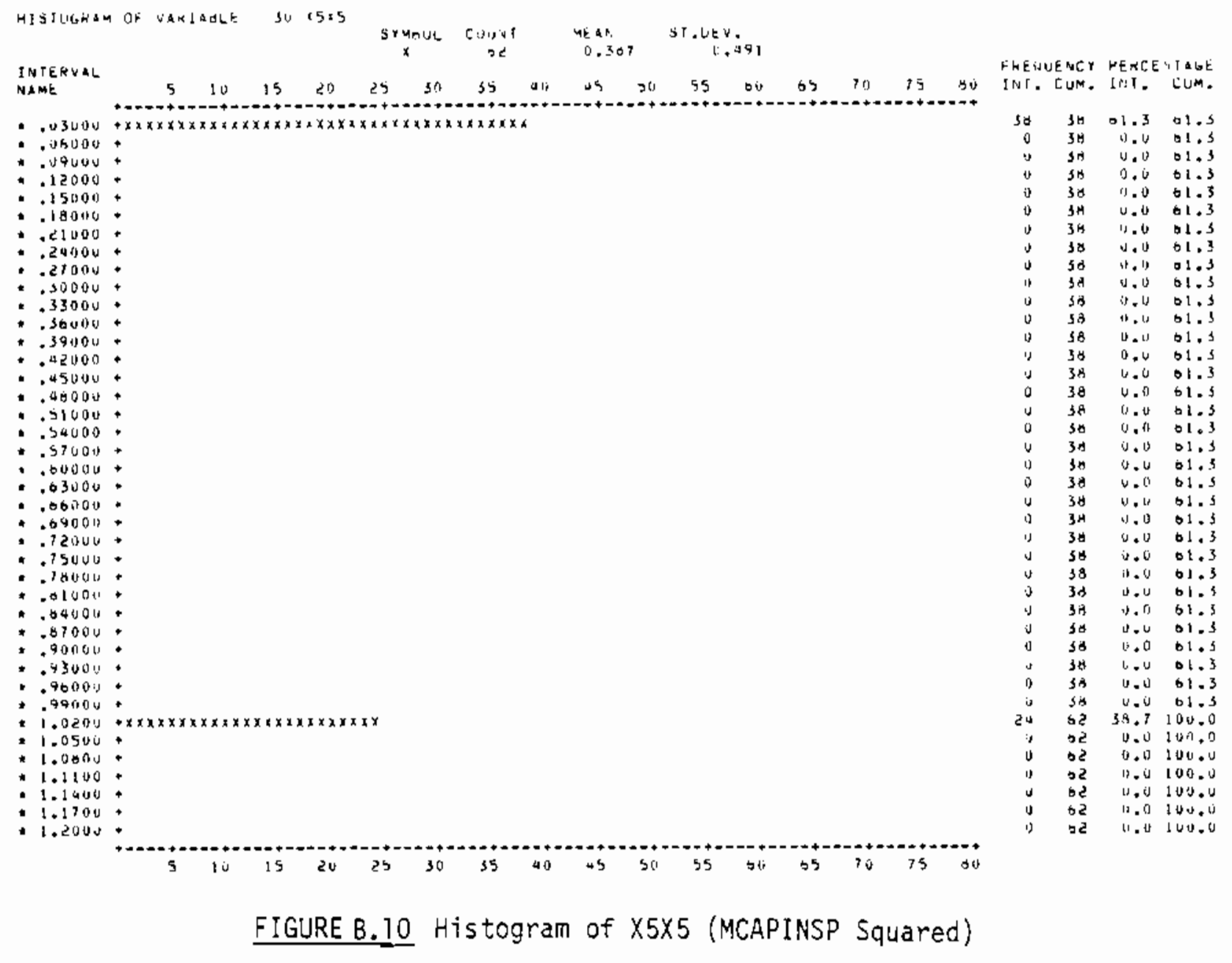

B. 11 


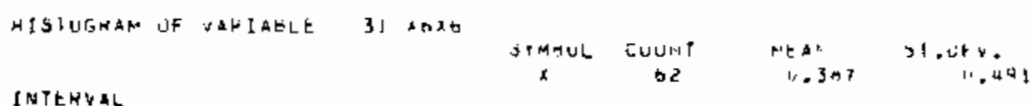

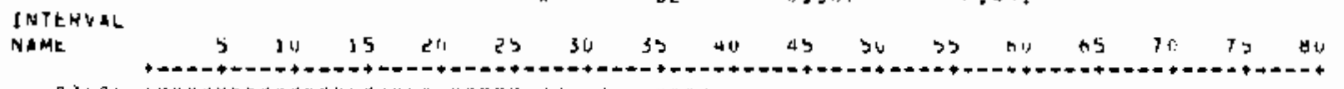

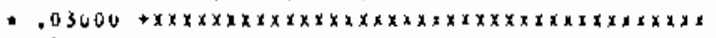

- ibuva.

$-49000+$

$12000+$

* 15000.

- Lacuo.

$-.21000$.

- zavora.

-.27000

- 30400.

-.\$30011.

- stcotio.

- 30

-

- 42001.

- 48004.

*.510u0.

$.54000+$

- .57000.

.60000.

-.0300u.

- $.60000+$

.. $.040 u 0$.

- rzvos.

- .750ua.

- trual.

- 8100 .

-

- . 44000.

- corbina.

-.40000.

-.9500.

-. .90000.

- $4900 \mathrm{r}$

- $1.0200+x \times x \times x \times x \times x \times x \times x \times x \times x \times x \times x \times x \times$

- 1.0500.

- $1.000 \mathrm{cos}$

1.1100.

-1.1000+

1.1700.

$1.2000 *$

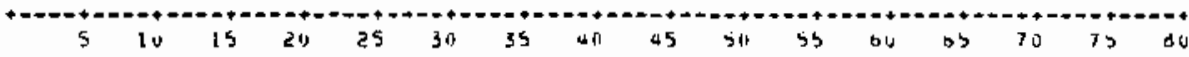

FHELUE YLT PEACEVIAGK

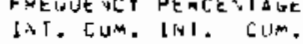

\begin{tabular}{|c|c|c|c|}
\hline 50 & 3H & D) 3 & 01.5 \\
\hline$v$ & 36 & u.u & S1. \\
\hline t) & 38 & (1. 12 & 61.5 \\
\hline v & 3 & 0.0 & 0.1 .5 \\
\hline 3 & $3 x$ & 0.0 & 61.3 \\
\hline$\checkmark$ & 56 & $v . v$ & 61.3 \\
\hline$v$ & $3 \mathrm{~B}$ & 4.5 & b). 3 \\
\hline$v$ & 34 & 0.0 & b1. 3 \\
\hline 0 & So & 0.0 & 01.5 \\
\hline$v$ & $3 \mathrm{~A}$ & 0.0 & 61.5 \\
\hline$v$ & $3 H$ & $v . v$ & 61.3 \\
\hline$v$ & 34 & $i t . v$ & 01.3 \\
\hline$\checkmark$ & 30 & 8.6 & $B 1, s$ \\
\hline$v$ & $3 t$ & 6.1 & $b 1 . y$ \\
\hline$v$ & 54 & $v .4$ & 01.5 \\
\hline$v$ & 38 & $n .0$ & $61 . x$ \\
\hline$\psi$ & $3 \mathrm{H}$ & 0.0 & 61.5 \\
\hline u & sot & 13.6 & b) +3 \\
\hline 6 & 38 & 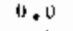 & 01.3 \\
\hline u & 38 & $v . v$ & b]. 5 \\
\hline 0 & 34 & v.u & $b 1.3$ \\
\hline 0 & $3 b$ & ט. 3 & 01.3 \\
\hline$v$ & $3 H$ & 0.11 & 01.3 \\
\hline$u$ & 38 & $\sqrt{a}$ & 61.3 \\
\hline 0 & 38 & $w . v$ & 01.5 \\
\hline$v$ & $3 \mathrm{H}$ & $0 . v$ & 0.1 .3 \\
\hline$v$ & $3 n$ & 0.0 & 61.3 \\
\hline$v$ & 38 & 10.0 & 01.3 \\
\hline 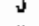 & 38 & 4.6 & $\$ 1.5$ \\
\hline$\varphi$ & 39 & 0.0 & 01.3 \\
\hline 0 & 58 & 0.4 & 01,3 \\
\hline$v$ & $3 n$ & 0.0 & 01.5 \\
\hline$v$ & 38 & 0.0 & 01.3 \\
\hline 24 & 62 & 38.7 & $(4) .4$ \\
\hline$v$ & 02 & 4.0 & $1 \cup 0,1)$ \\
\hline$v$ & 62 & 1). & $1 \cup 4.5$ \\
\hline 0 & $b 2$ & 2.0 & $1 \cup \cup . d$ \\
\hline 4 & 62 & 0.4 & 100.11 \\
\hline 6 & 62 & v. v & 1 し \\
\hline 1) & 62 & 0.4 & 100 \\
\hline
\end{tabular}

FIGURE 8.11 Histogram of $\times 6 \times 6$ (Gamma Squared) 


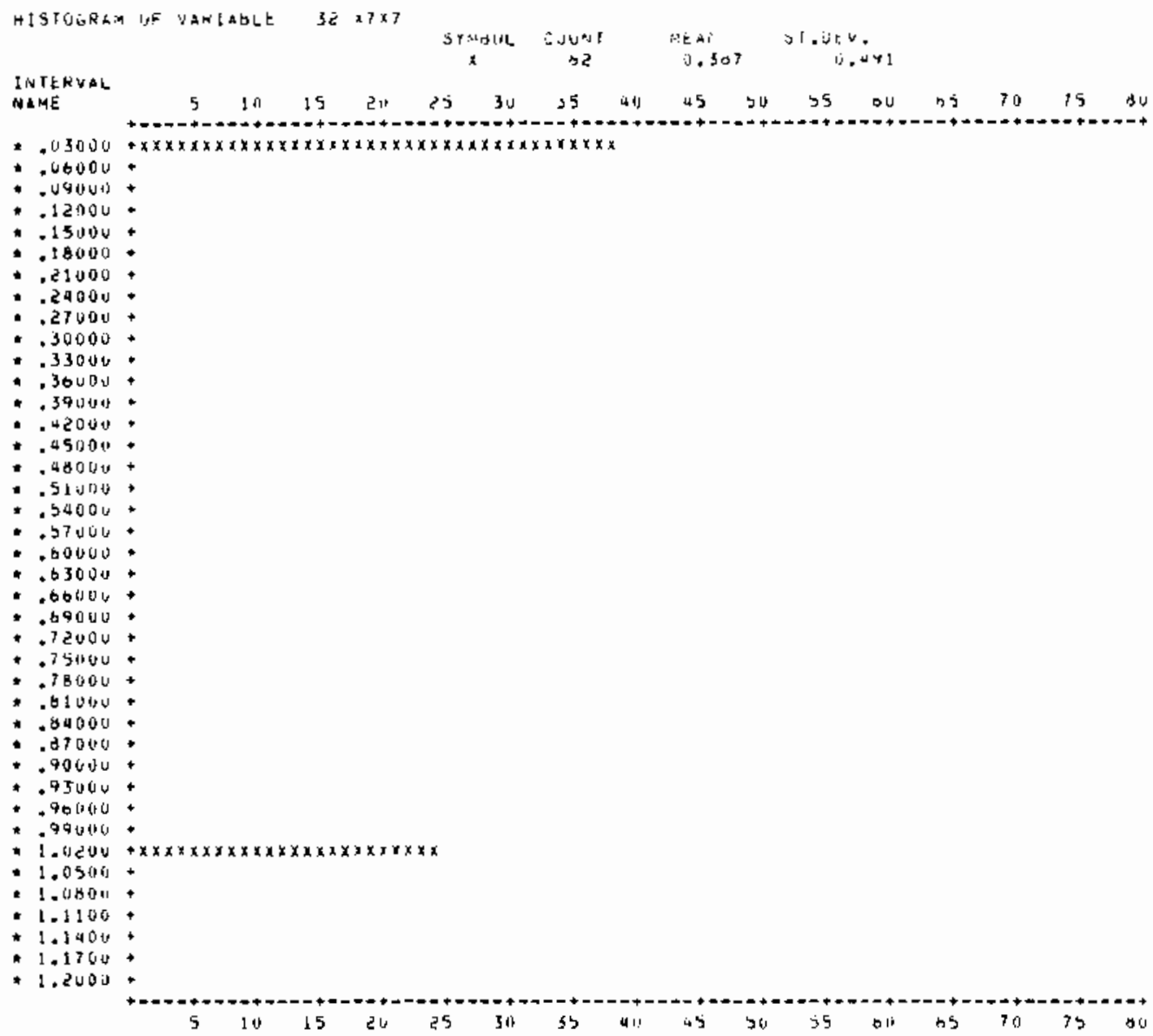

FMEIJ)ENCY HERE, VIALE INI. CUM, INT, CUNi.

- .0600u

- v90uas.

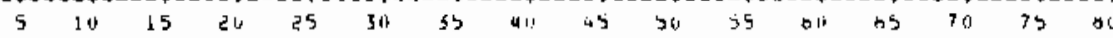

301.301 .3

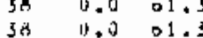

st 0.0 bl.

38 0.0 b..

35 13.0 61.3

3] 0.061 .3

IA 0.0 b.

38 o. 01.3

36 d. 01.3

5 H 0.0 01.3

38 0.0 का.

3 म 0.401 .5

38 u.j ol.

उस v. की ०1.5

sh u. bl bl.

उ० 0.0 ०ा.

58

SA U.

ja

38 . 01 .

so 0.001 .3

30 b 1.0

34 प. 0 क

3 H th

38 0 ov bi:

sa va bi.

so 19.0 bl. 3

3a 1.0 o. 1.3

30 v. b bो.

38 o. 0 ol.

उत

कर 30.71340

be jo.r jug

or a. 100.

०ट प.र 140,4

मर 1.0 iv

10.0100 .0

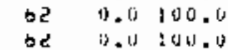

FIGURE 8.12 Histogram of $X 7 \times 7$ (Throughput Squared) 


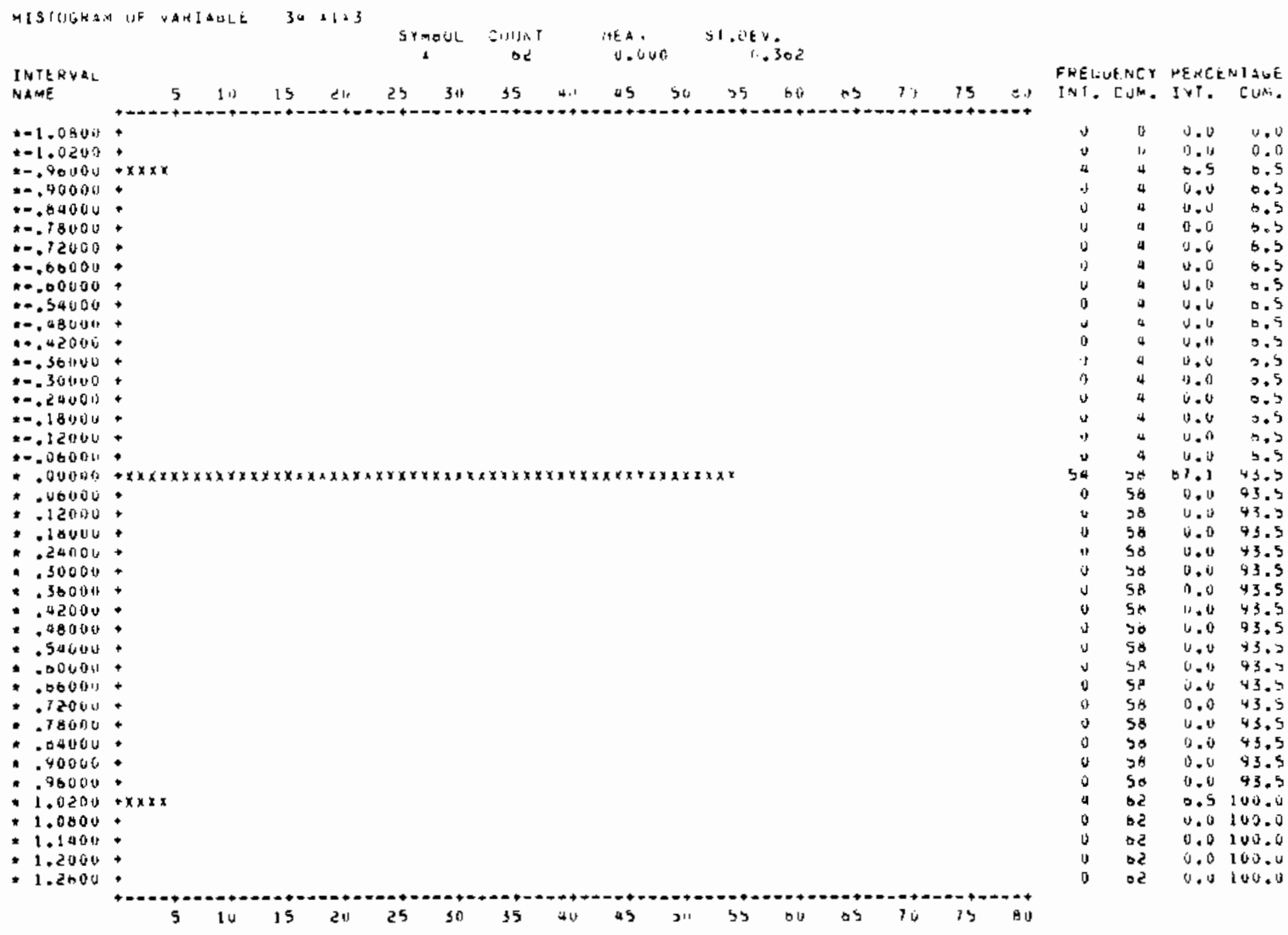

FIGURE B.13 Histogram of $X 1 \times 3$ (Alpha Times GQ) 


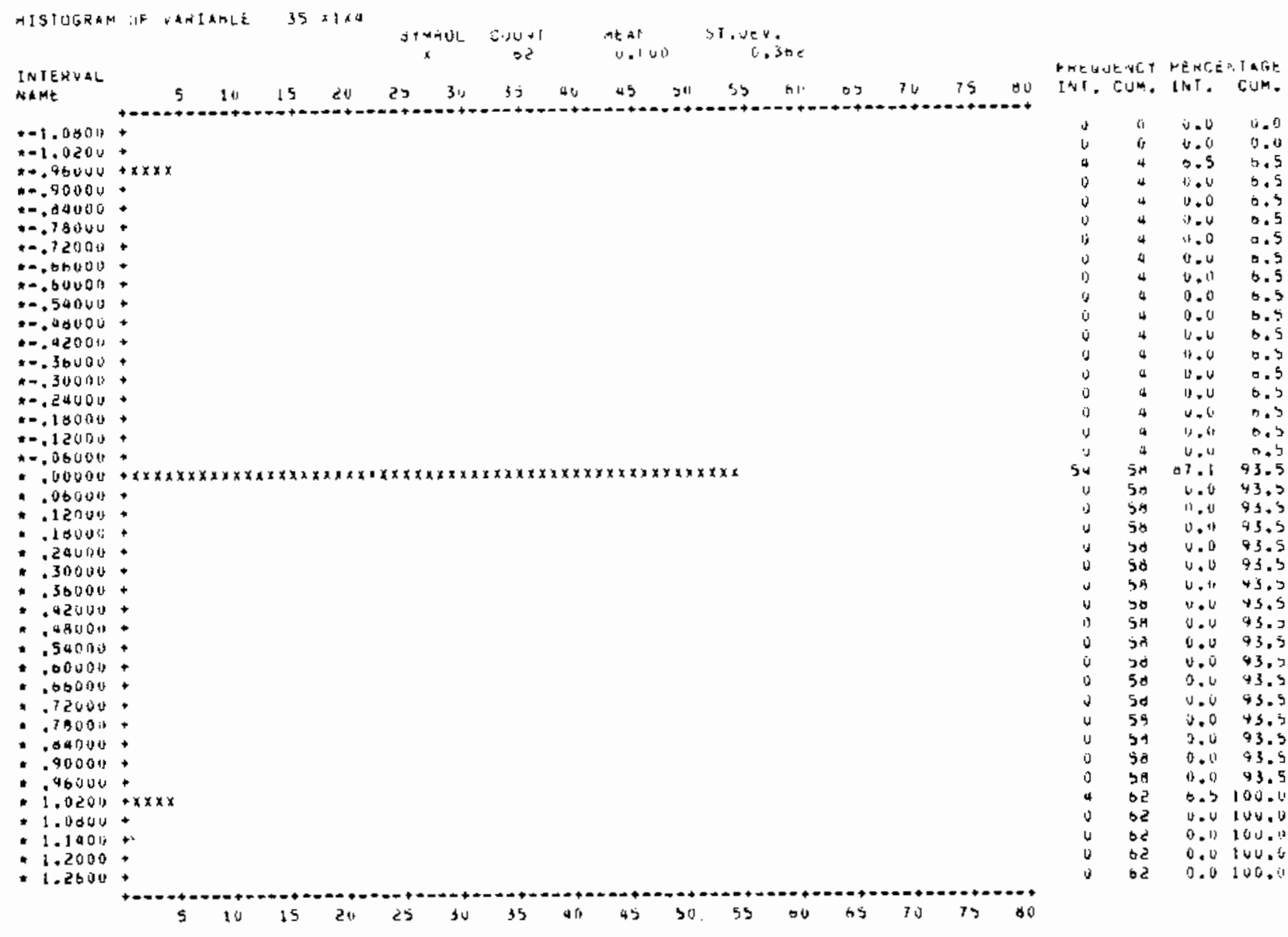

FIGURE B.14 Histogram of X1X4 (Alpha Times MCAPOP) 


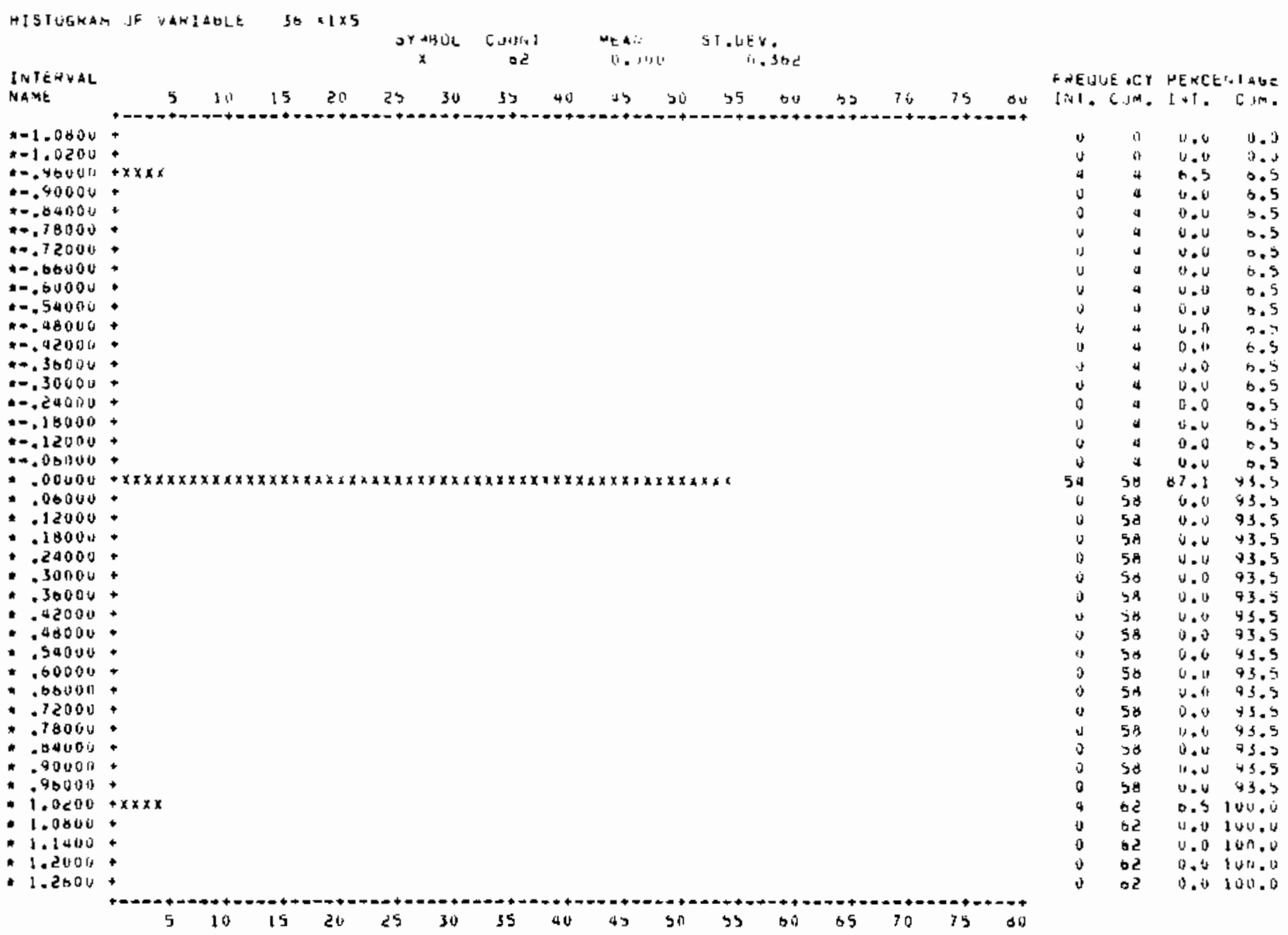

FIGURE B.15 Histogram of $X 1 \times 5$ (Alpha Times MCAPINSP) 


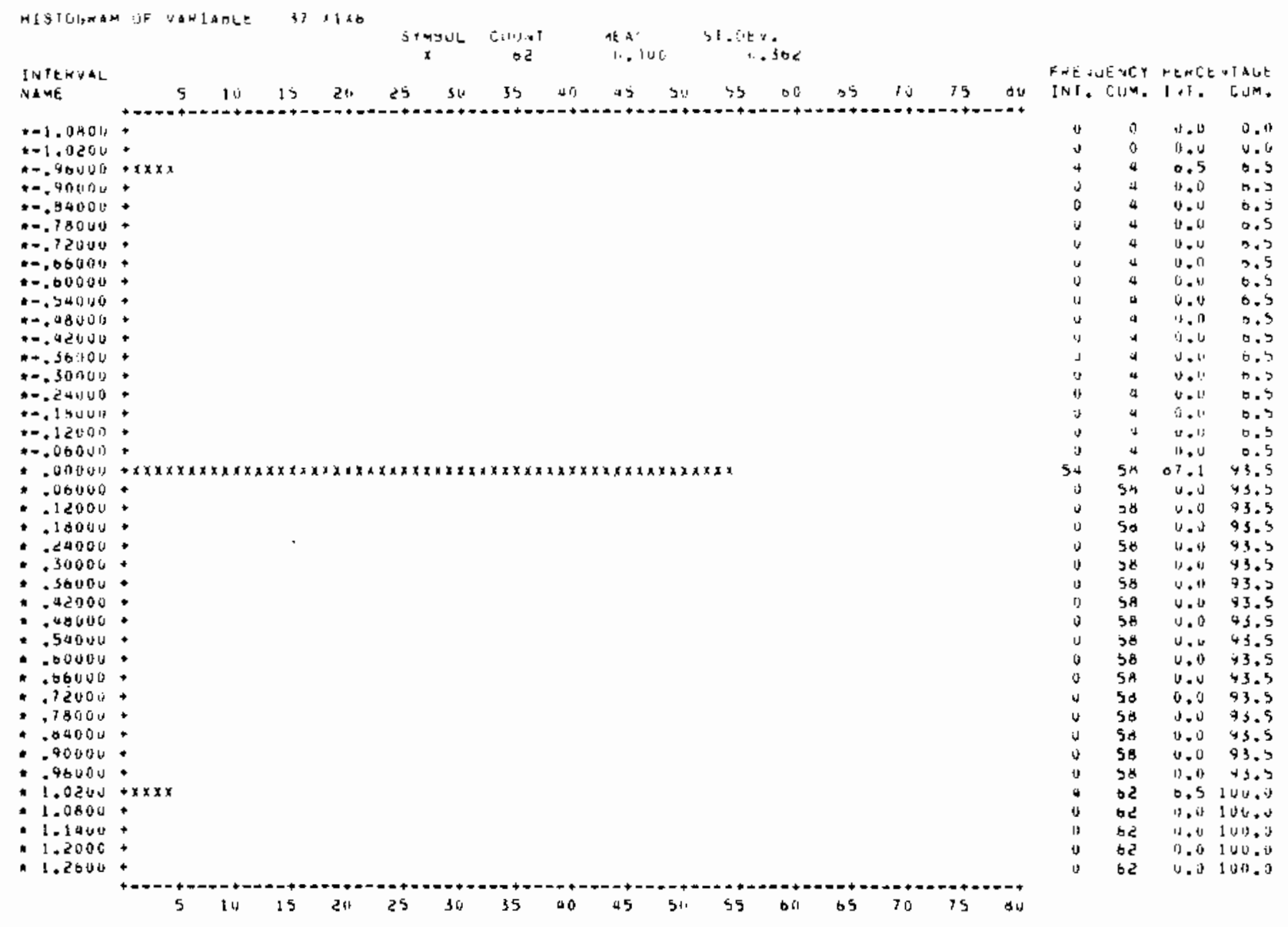

FIGURE B.16 Histogram of X7X6 (A1pha Times Gamma) 


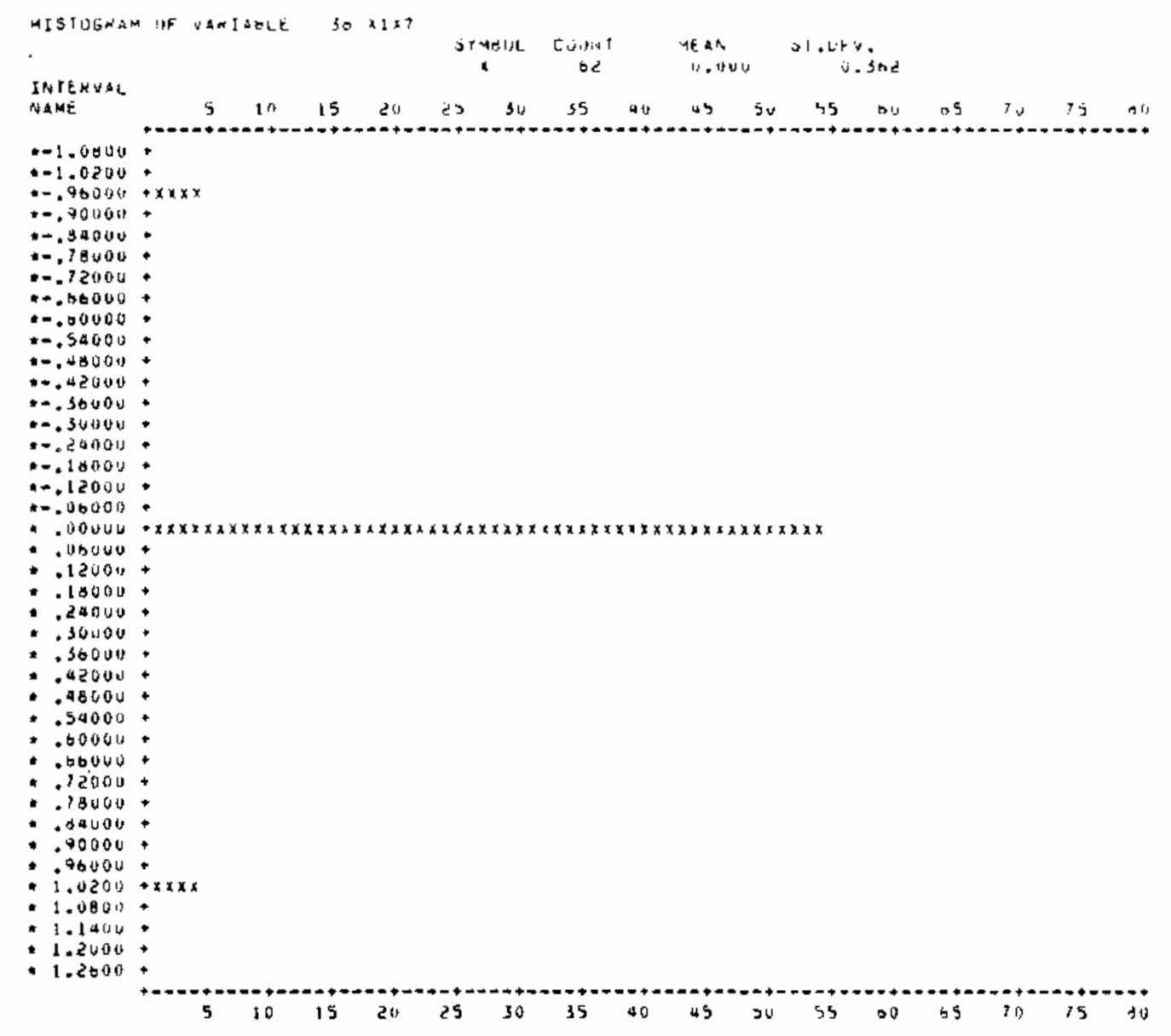

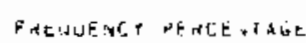
I:T. LU:T. IVI. LIJM.

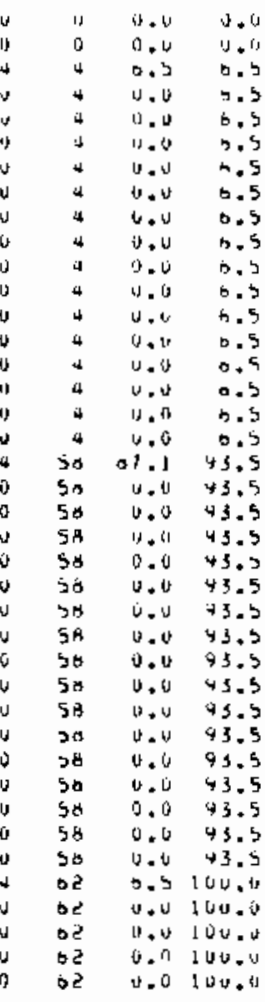

FIGURE B.I7 Histogram of $\times 1 \times 7$ (Alpha Times Throughput)

B. 18 


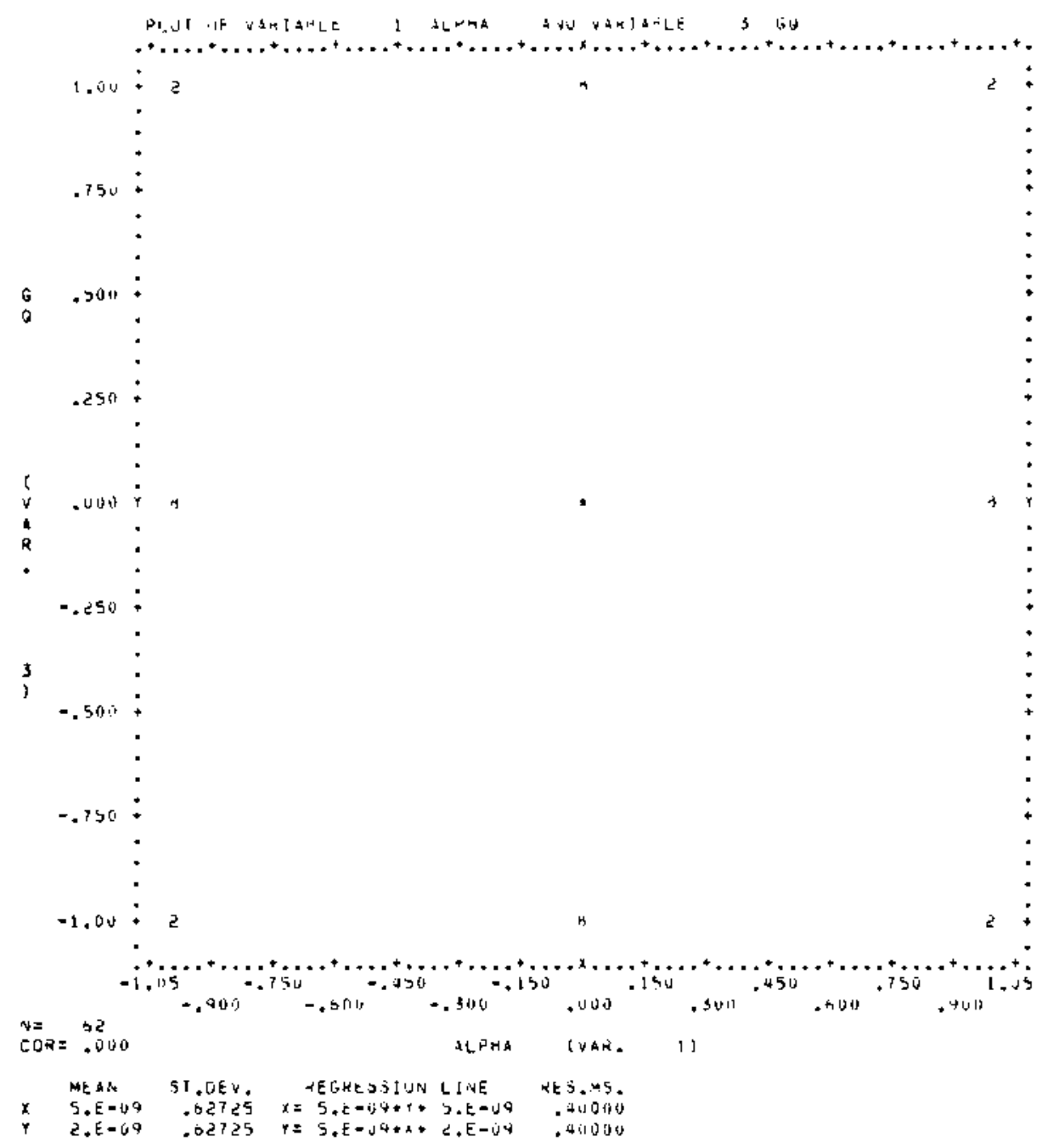

FIGURE B. 18 Goal Quantity Versus Alpha 


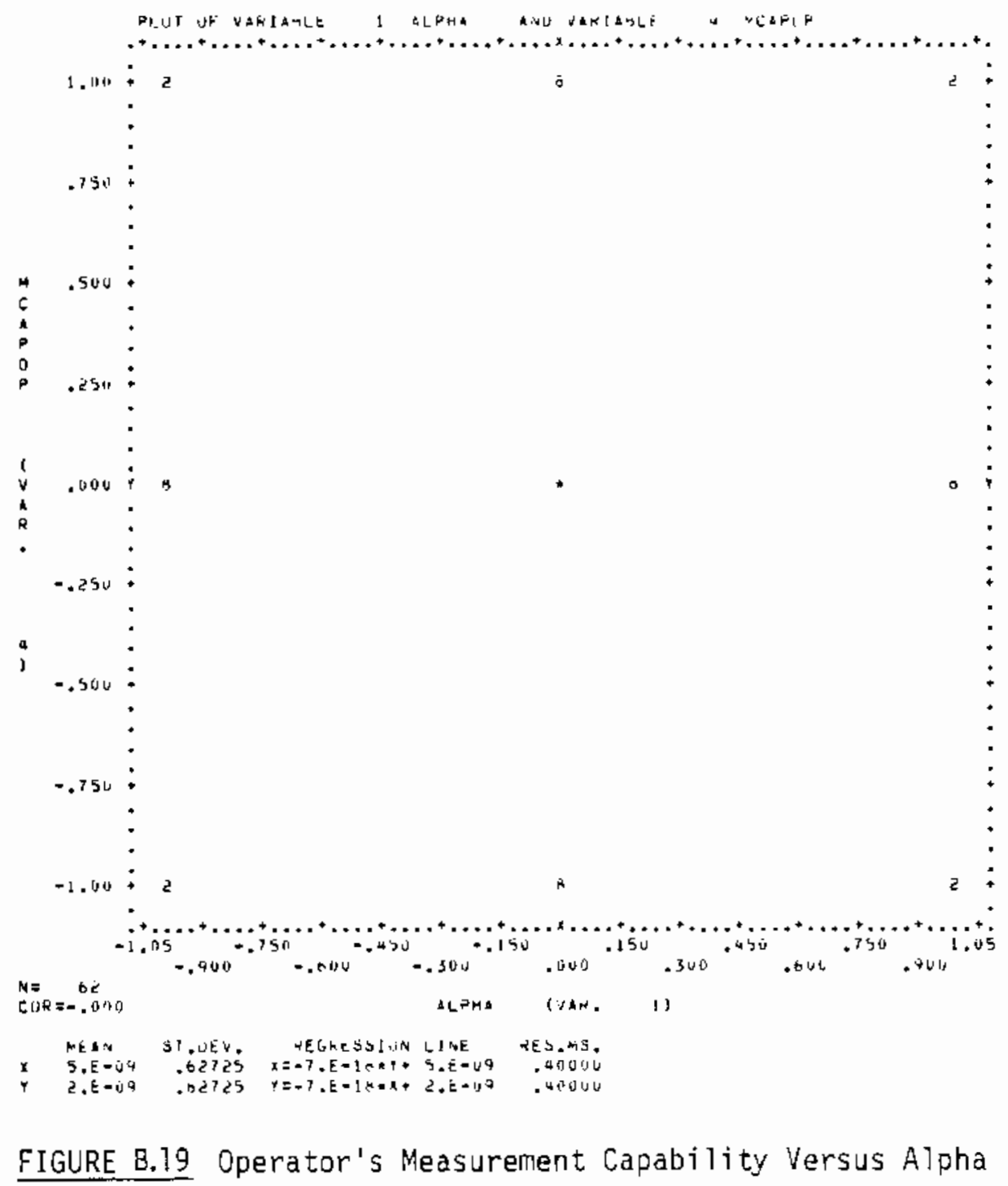




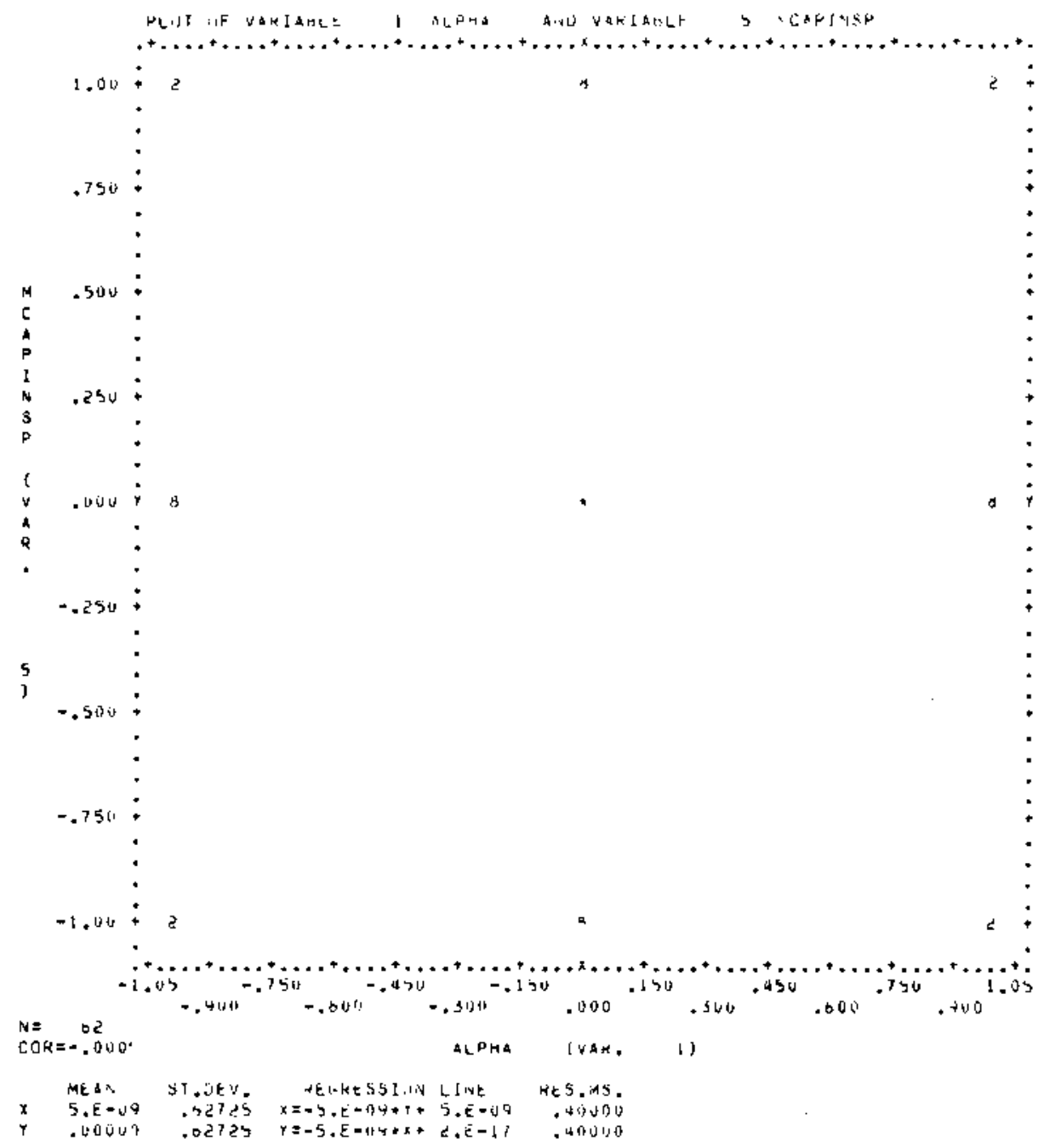

FIGURE B.20 Inspector's Measurement Capability Versus Alpha 


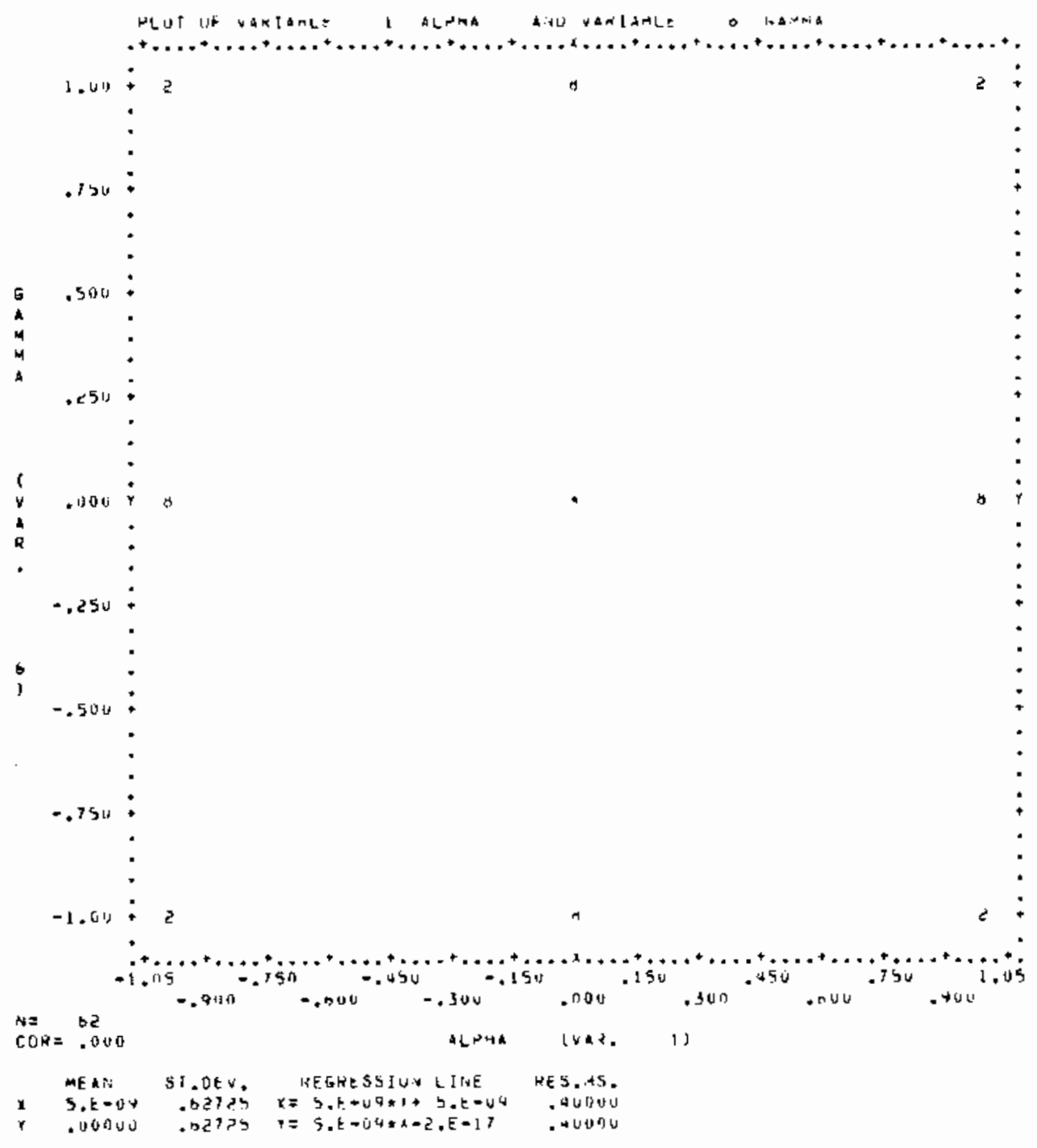

FIGURE B.21 Crossover Point Versus A]pha 


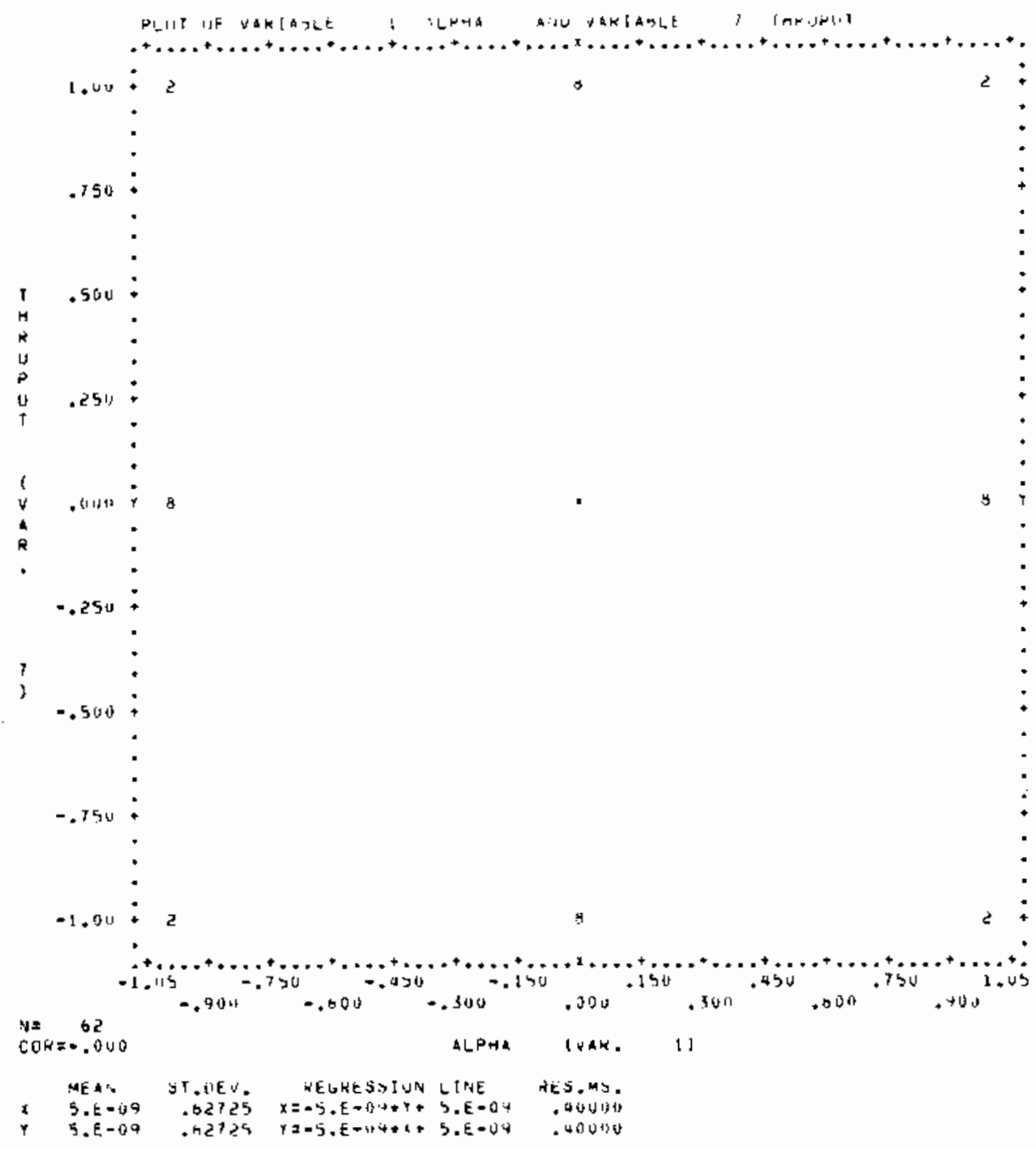

FIGURE B.22 Throughput Versus Aipha 
of the experimental region, eight cases on each of the four sides, and the remaining 22 cases at the center point. The variables are uncorrelated (this is the meaning of $\mathrm{COR}=.000$ printed under the plot) and the experimental pattern is obviousiy balanced and symmetric. The symmetry is actualiy circular about the center point although this is perhaps not obvious from the plot. (The corners, each having two points, are equidistant from the center point; the sides, each having eight points, are also equidistant from the center point.) This is characteristics of Box-Behnken designs, i.e., they are "rotatable."

A11 the other plots of first order inputs versus other first order inputs follow the same pattern. They have not been included in this report. 
APPENDIX C

\section{GRAPHS AND ANALYSIS OF RESPONSE VARIABLES}

There were many other figures analyzed than those shown as Figures 13 through 17. Several are shown here with accompanying explanations.

Figure $C .1$ shows a plot of $Q_{M A X}$ versus beta, the goal probability of nondetection. Again, there appears to be little relationship between the variables. In other words, the actual probability of nondetection bears little relationship to the goal probability of nondetection. The explanation is that for most of the 62 cases the goal probability was unattainable for various reasons (e.g., large throughput, insufficient measurement quality), and the actual achieved probability was driven by other factors.

Figure $C .2$ shows side-by-side histograms of $\mathrm{Q}_{\text {MAX }}$ for the three levels of beta. Again, there does not appear to be much of a relationship.

Figure C.3 shows a plot of $Q_{\text {MAX }}$ versus goal quantity (GQ). The relationship here is a little stronger than was seen in Figures 14 and $C .1$. Visually there appears to be a negative association between goa $\mathrm{f}$ quantity and $Q_{M A X}$. In other words, the larger the goal quantity, the smaller Q $Q_{\text {MAX }}$, and thus the larger the probability of detection. This result is not surprising: it is obvious that the larger the amount diverted, the higher the probability of detection.

Figure C.4 shows side-by-side histograms of $Q_{\text {MAX }}$ for the three levels of goal quantity $(G Q)$. The same negative correlation can be seen in a somewhat different light.

Figure 0.5 shows $Q_{\text {MAX }}$ versus the operator's measurement capability (denoted by MCAPOP in the figure). There is little apparent relationship between the two. This is somewhat surprising in that it says that the probability of detection bears no relation to the quality of the operator's measurements. This conclusion is correct, given the assumptions on which the mathematical model is based, but the assumptions include a certain minimum level of accountancy performance by the operator. For example, the operator needs to maintain measurement systems in control, with no by- 


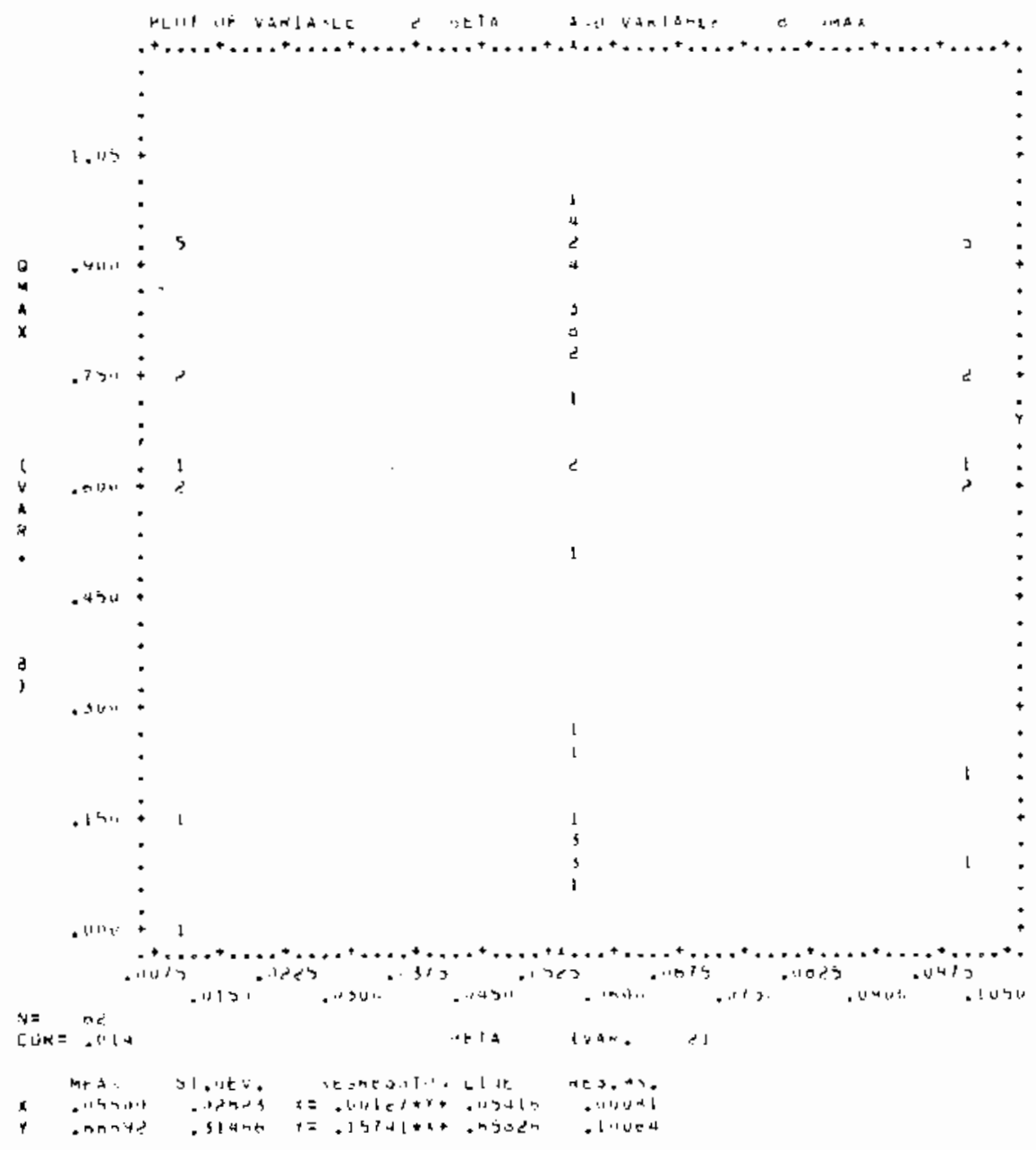

FIGURE C.I Q QAX Versus Beta 


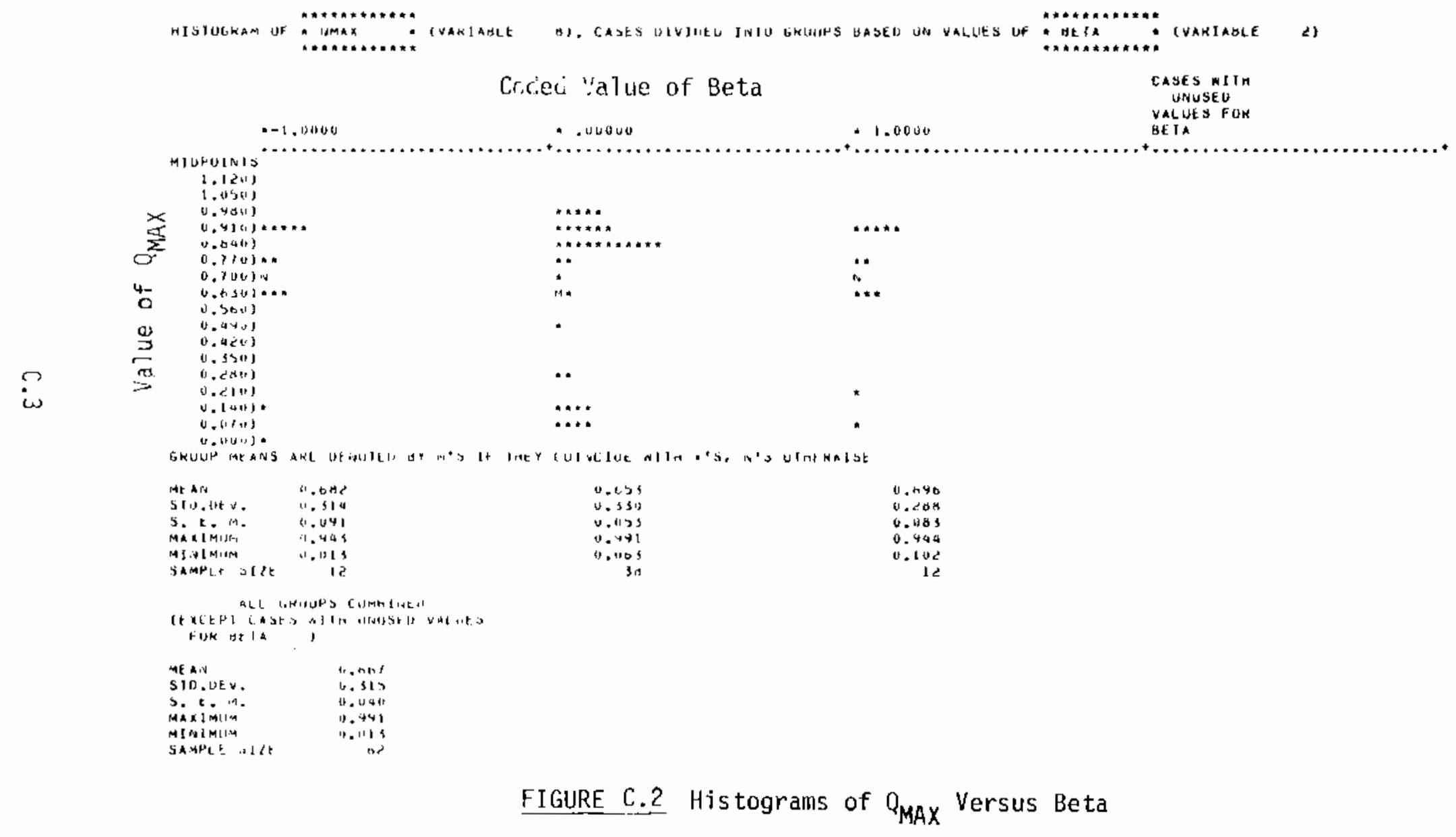




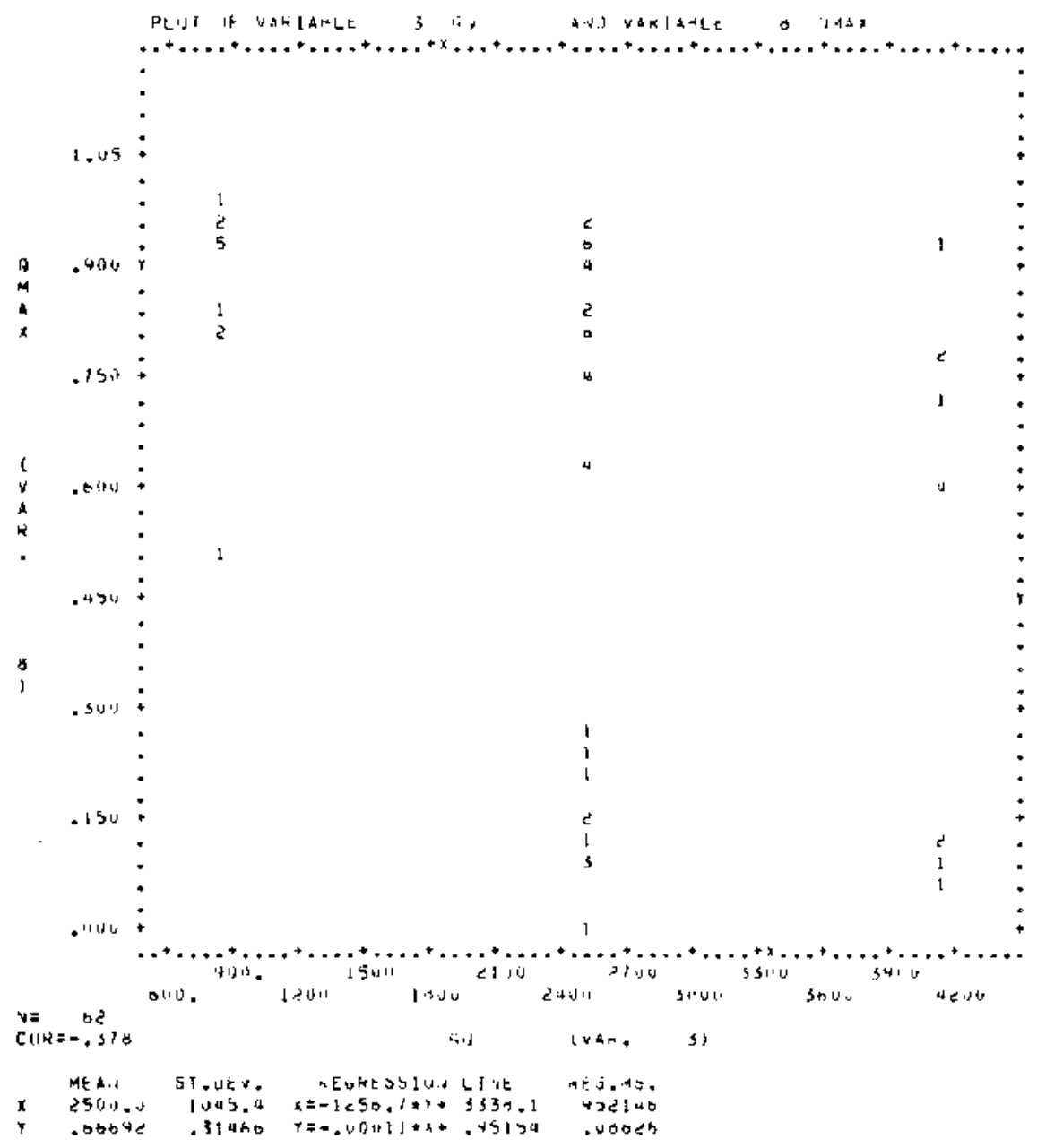

FIGURE C.3 Q Q MAX Versus Goal Quantity 


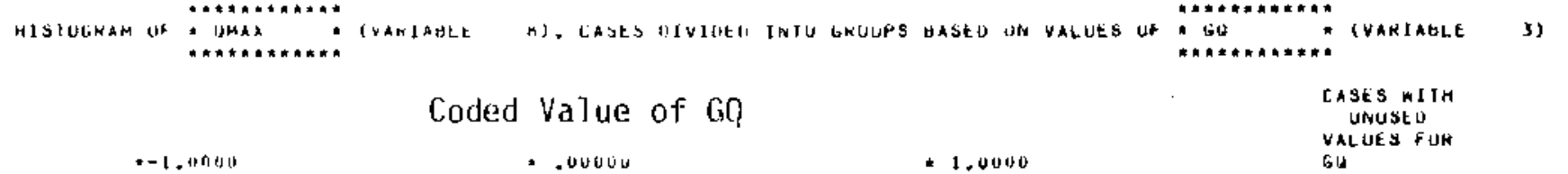

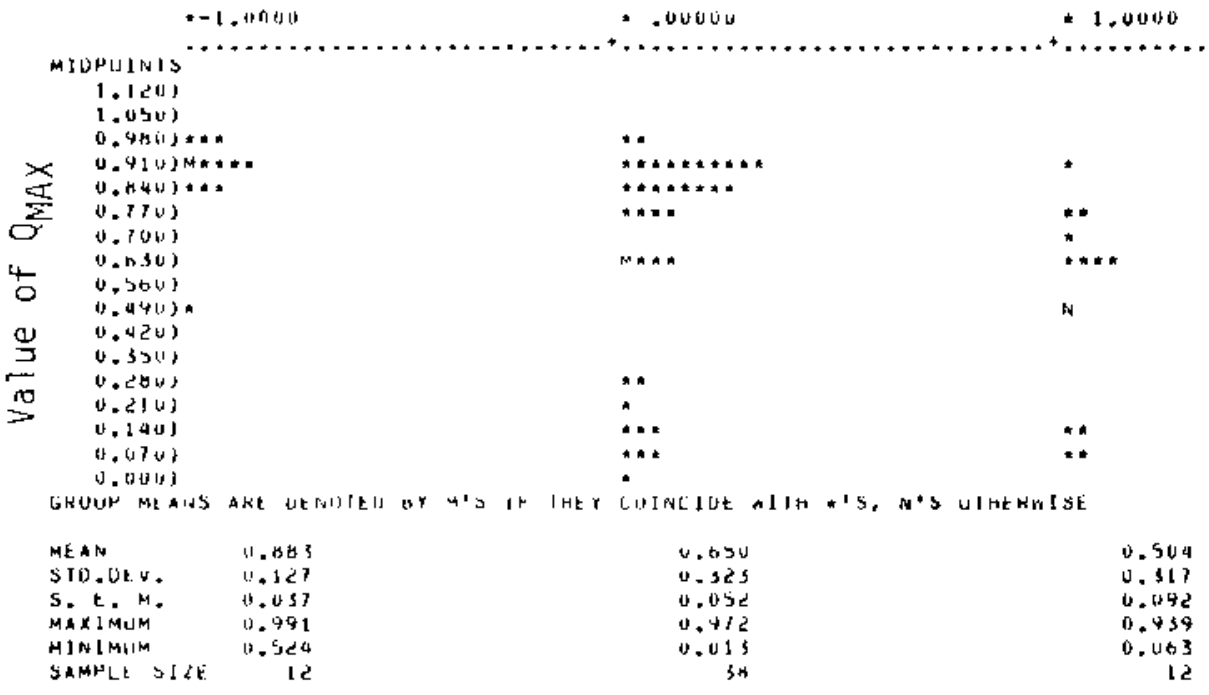

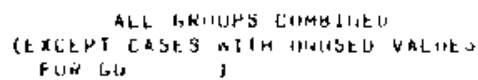

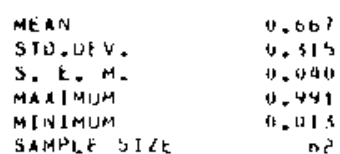

FIGURE C.4 Histograms of $Q_{M A X}$ Versus Goal Quantity 


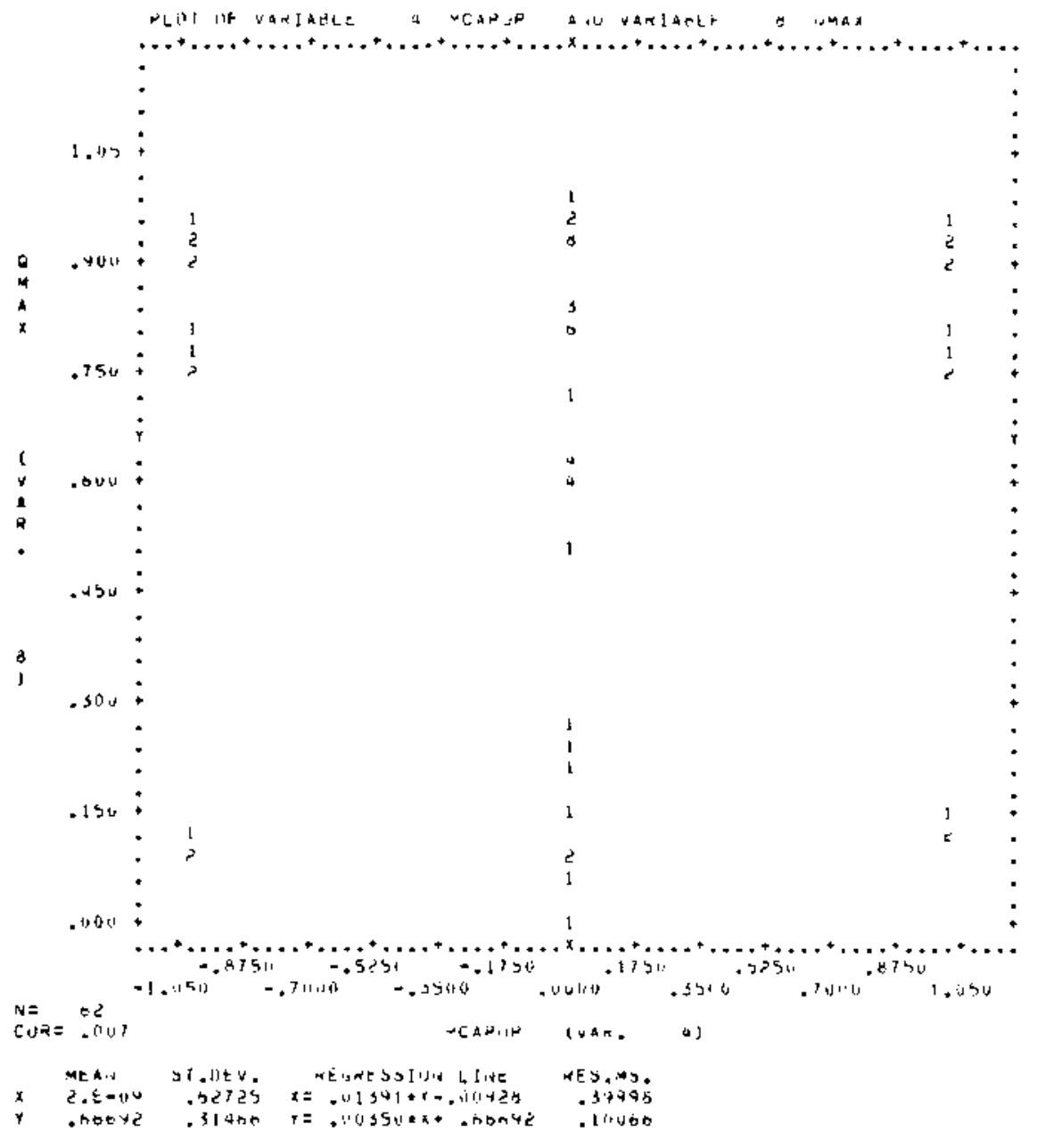

FIGURE C.5 QMaX Versus Operator's Measurement Capability 
difference accounting, no unmeasured material (holdup or sidestreams), etc. If these assumptions are satisfied, then the effectiveness of the verification depends primarily on the inspector's measurement capability rather than the operator's. This effect was discussed in the text and shown in Figures 13 through 17.

Figure $C .6$ is a scatter plot of $Q_{\text {MAX }}$ versus gamana, the crossover point. Recall that gamma is related to the accuracy of the inspector's attribute test instruments. Figure $\mathrm{C.6}$ seems to indicate that the crossover point has an effect on inspection effort. In other words, the primary impact of improved attributes measurements is on efficiency rather than effectiveness.

Figure $C .7$ is a scatter plot of $Q_{\text {Max }}$ versus the annual plant throughput (labeled THRUPUT in the figure). Visually, a moderately strong relationship is evident, not as strong as the relationship with the inspector's measurement capability, but stronger than any of the others. As the plant throughput increases from 200 to 600 tons per year, the probability of nondetection tends to increase, and the probability of detection decreases.

Figure $C .8$ shows side-by-side histograms of $Q_{\text {Max }}$ for each of the three levels of throughput. Again, the trend is clear, al though there is still a lot of variation not explained by throughput.

The remaining figures in this appendix deal with the output variable Total cost. The most important figures were shown in the text as Figures 18 through 22.

Figure $C .9$ shows a piot of Total Cost versus alpha, the false alarm probability. Figure C.10 shows side-by-side histograms of Total cost for the three levels of alpha. No clear relationship is evident for the range of values of alpha considered in this study.

Figure C.11 shows a plot of Total cost versus beta, the goal probability of non-detection; Figure $\mathrm{C} .12$ shows histograms of Total cost for each of the three levels of beta. There appears to be a negative association: as the goal probability of non-detection increases (i.e., as the goal probability of detection decreases), the cost of routine inspections decreases slightiy. The effect is not very large for the range of values of beta considered and 


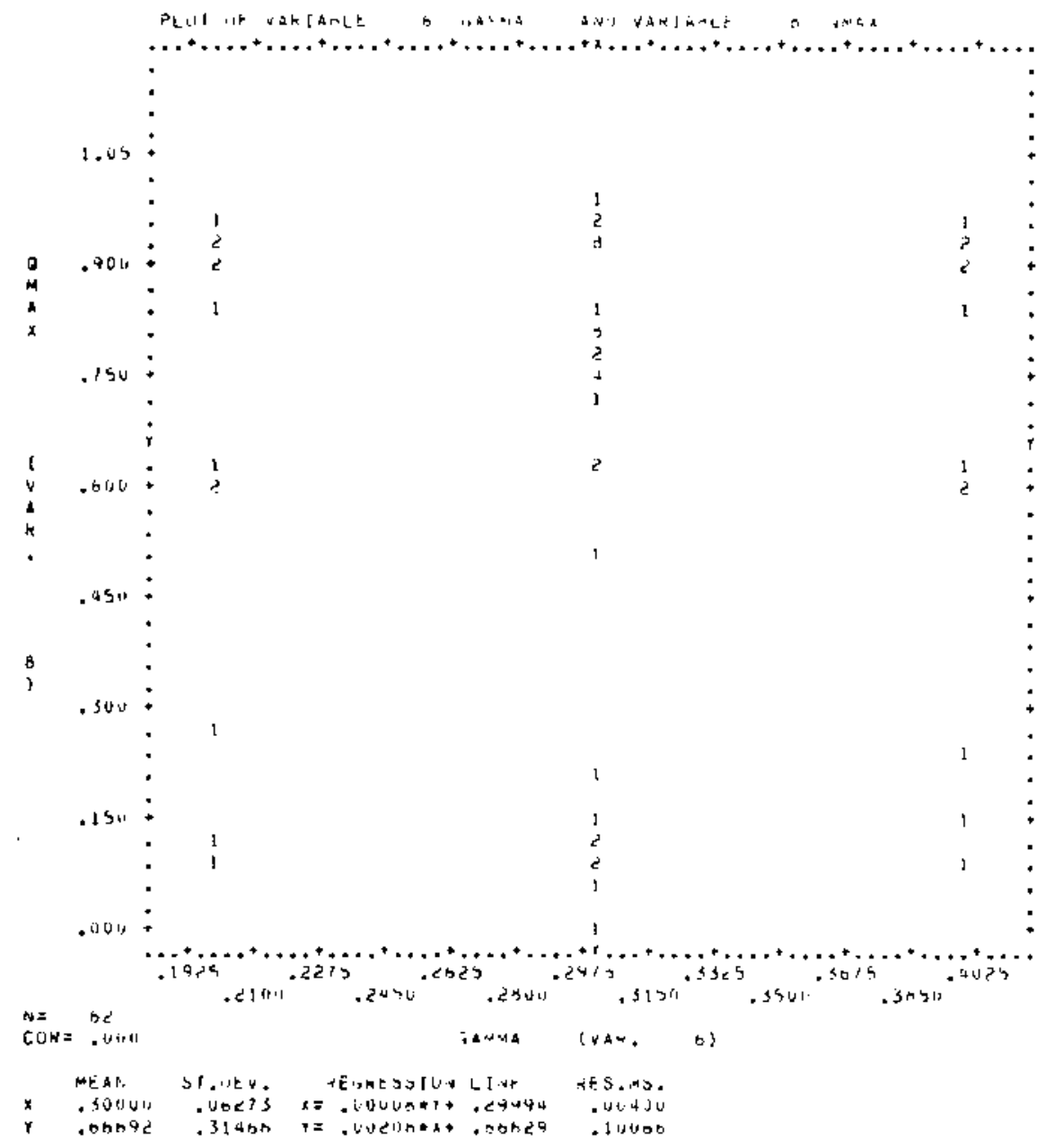

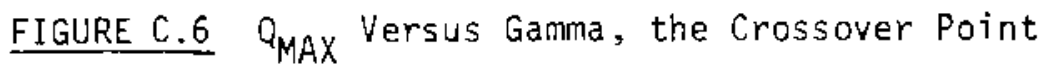




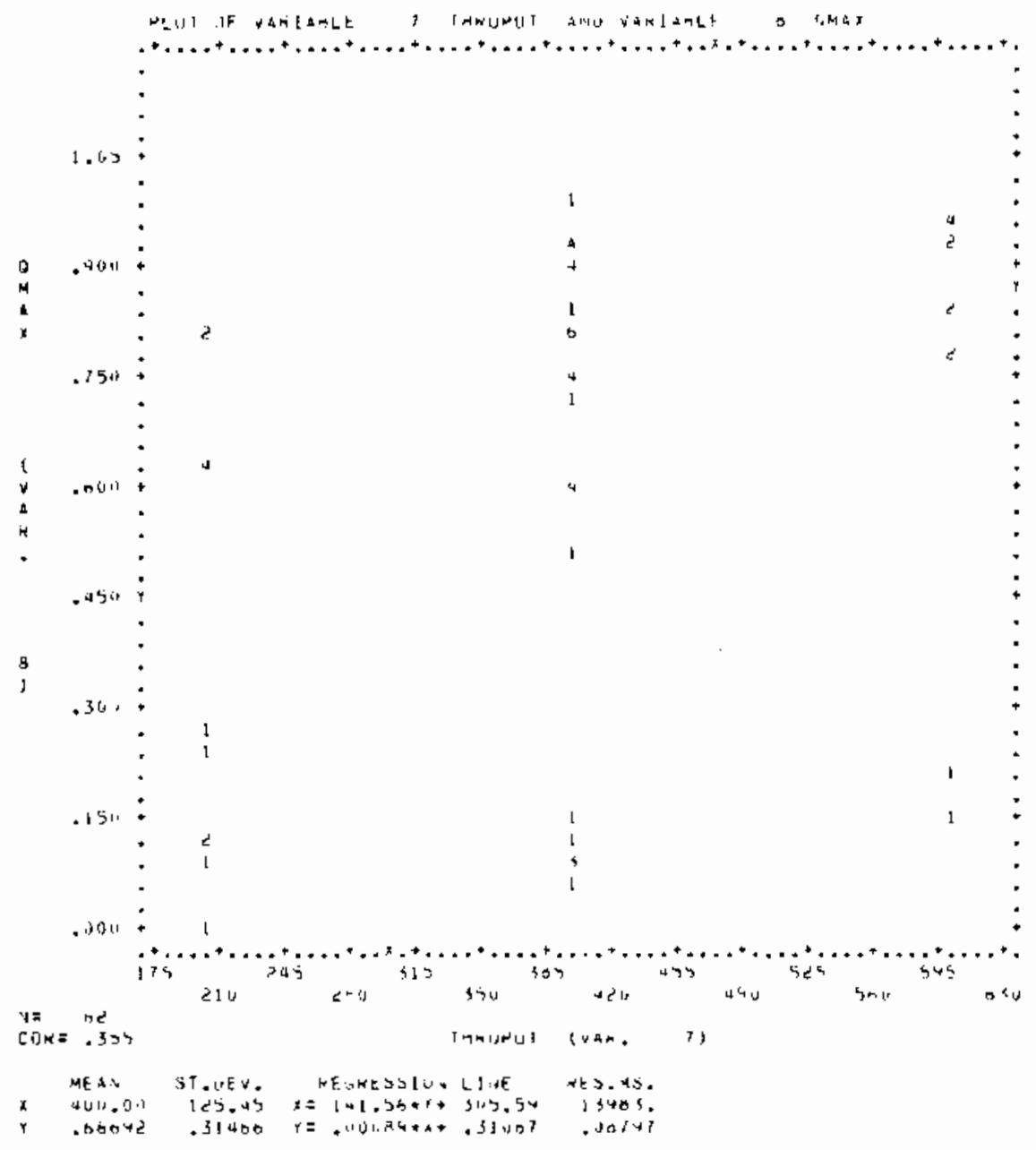

FIGURE C.I Q QAX Versus Throughput 


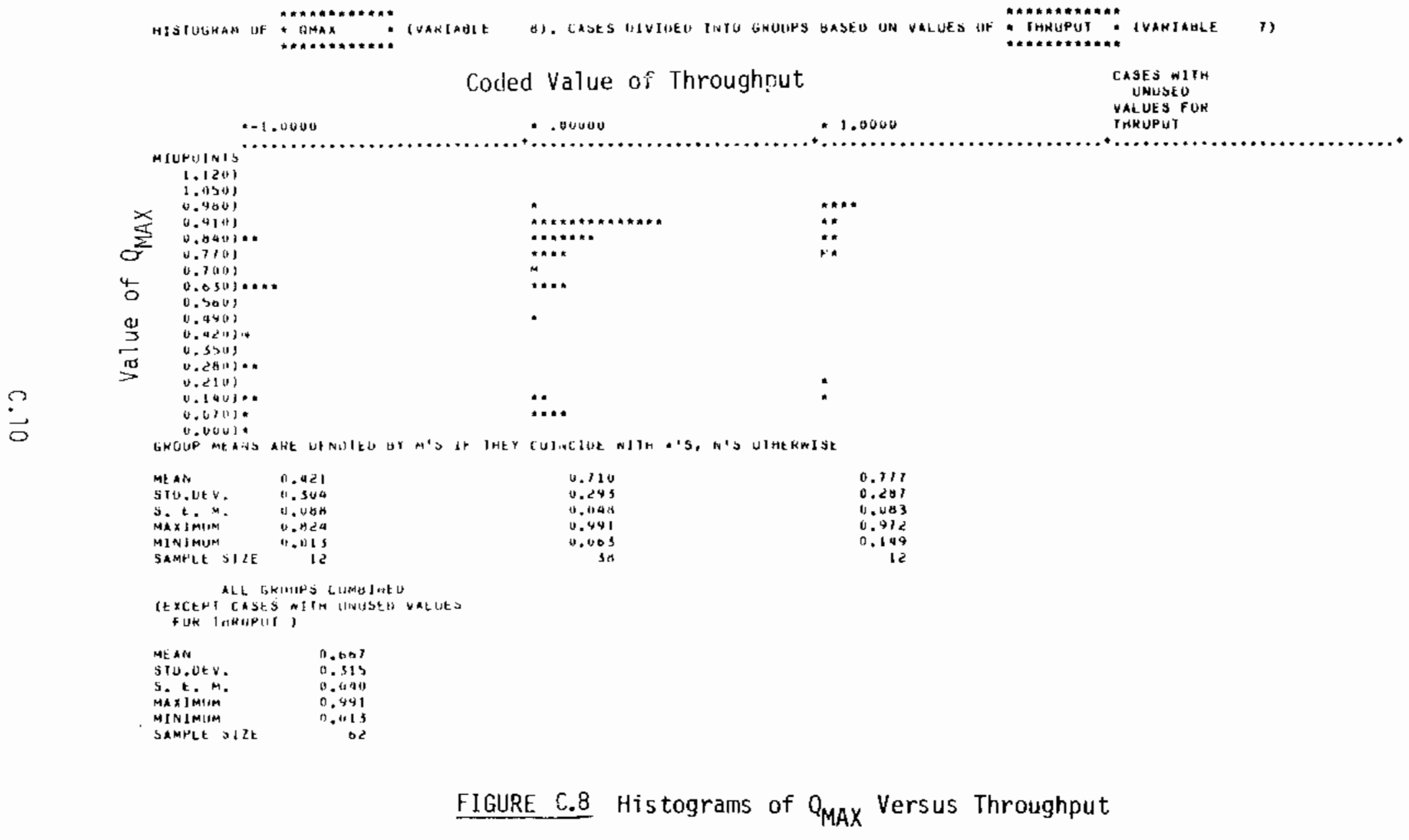




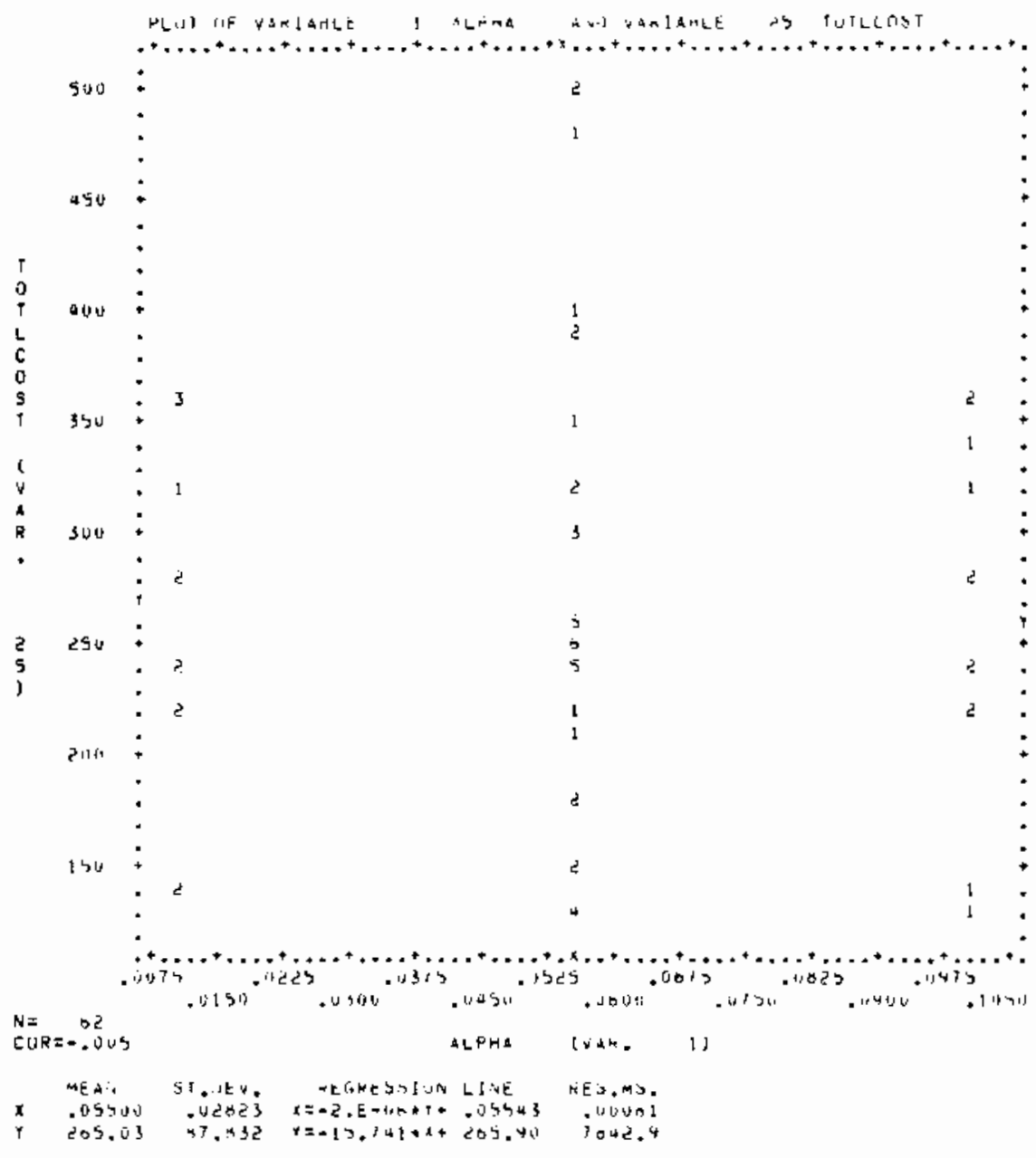

FIGURE C.9 Total cost Versus Alpha 


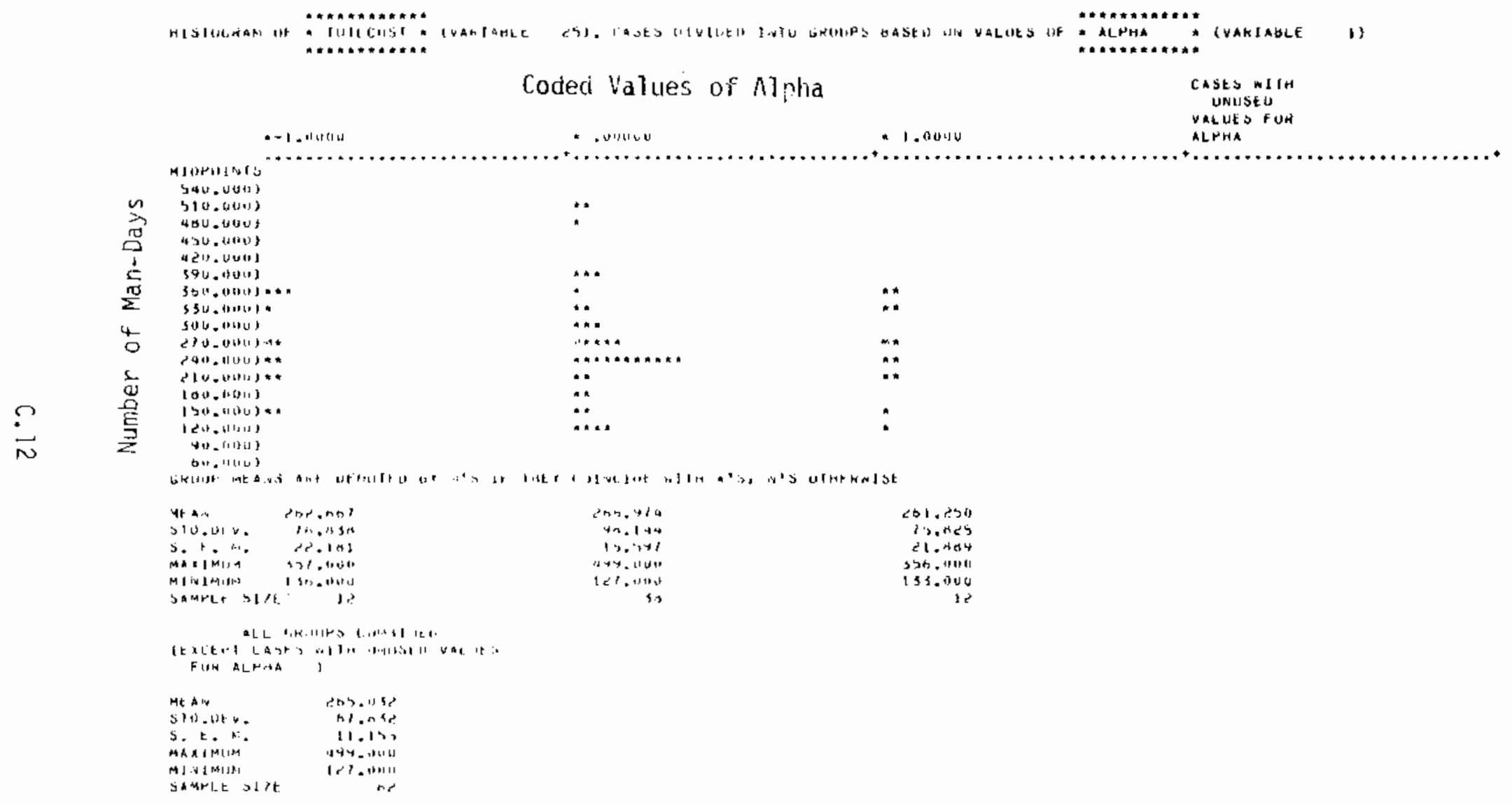

FIGURE C.10 Histograms of Total Cost Versus Alpha 


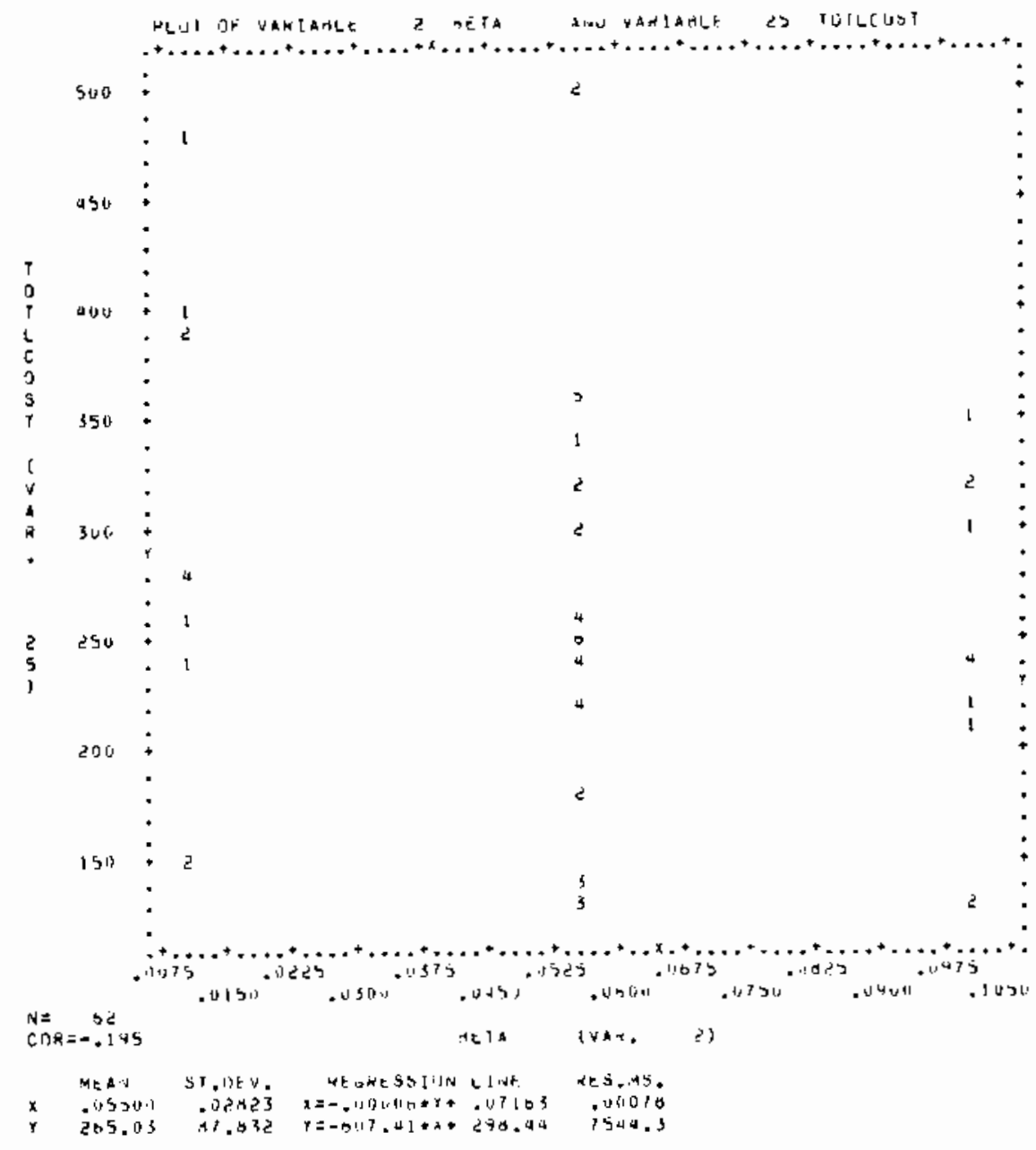

FIGURE C.11 Total Cost Versus Beta 


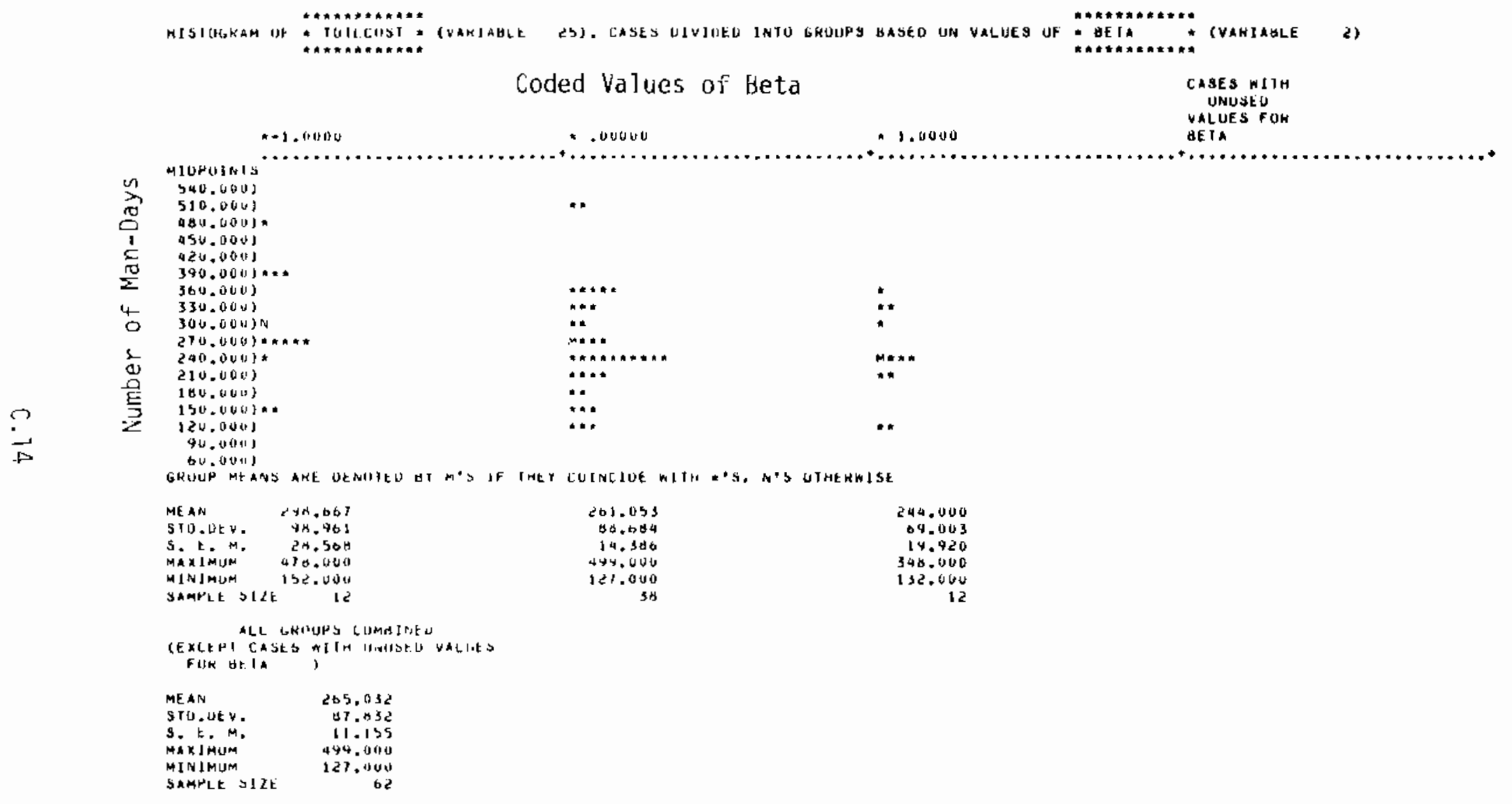

FIGURE C.12 Histograms of Total Cost Versus Beta 
it is obvious that other factors must be taken into account as weil, but the trend is clear.

Figure $C .13$ shows a piot of Total Cost versus the operator's measurement capability; Figure $C .14$ shows histograms of Total cost for the three levels of operator measurement capability. No relationship is apparent. As with $Q_{\text {MAX }}$, it should be noted that the operator's measurement capability refers to measurement error standard deviations only, and not to other aspects of the facility's or the state's system of accounting and control. These other aspects of the system can have a strong impact on the effort required for routine inspections, but they are not included in the analysis.

Figure $C .15$ shows a plot of Total cost versus the inspector's measurement capability; Figure $\mathrm{C} .16$ shows histograms of Total Cost for the three levels of inspector's measurement capability. No relationship is apparent. This contrasts with the strong effect that the inspector's measurement capability exerted on $Q_{\text {MAX }}$.

Figure $C .17$ shows a plot of Total Cost versus gama, the crossover point; Figure $C .18$ shows histograms of Total Cost for the three levels of gamma. As gamma increases (i.e., as the accuracy of the inspector's attributes test instrument decreases), the total cost tends to increase slightly. Recall that garma had no impact on effectiveness $\left(Q_{M A X}\right)$. Here we see that gamma does indeed have some (slight) impact on efficiency, as measured by Total cost. 


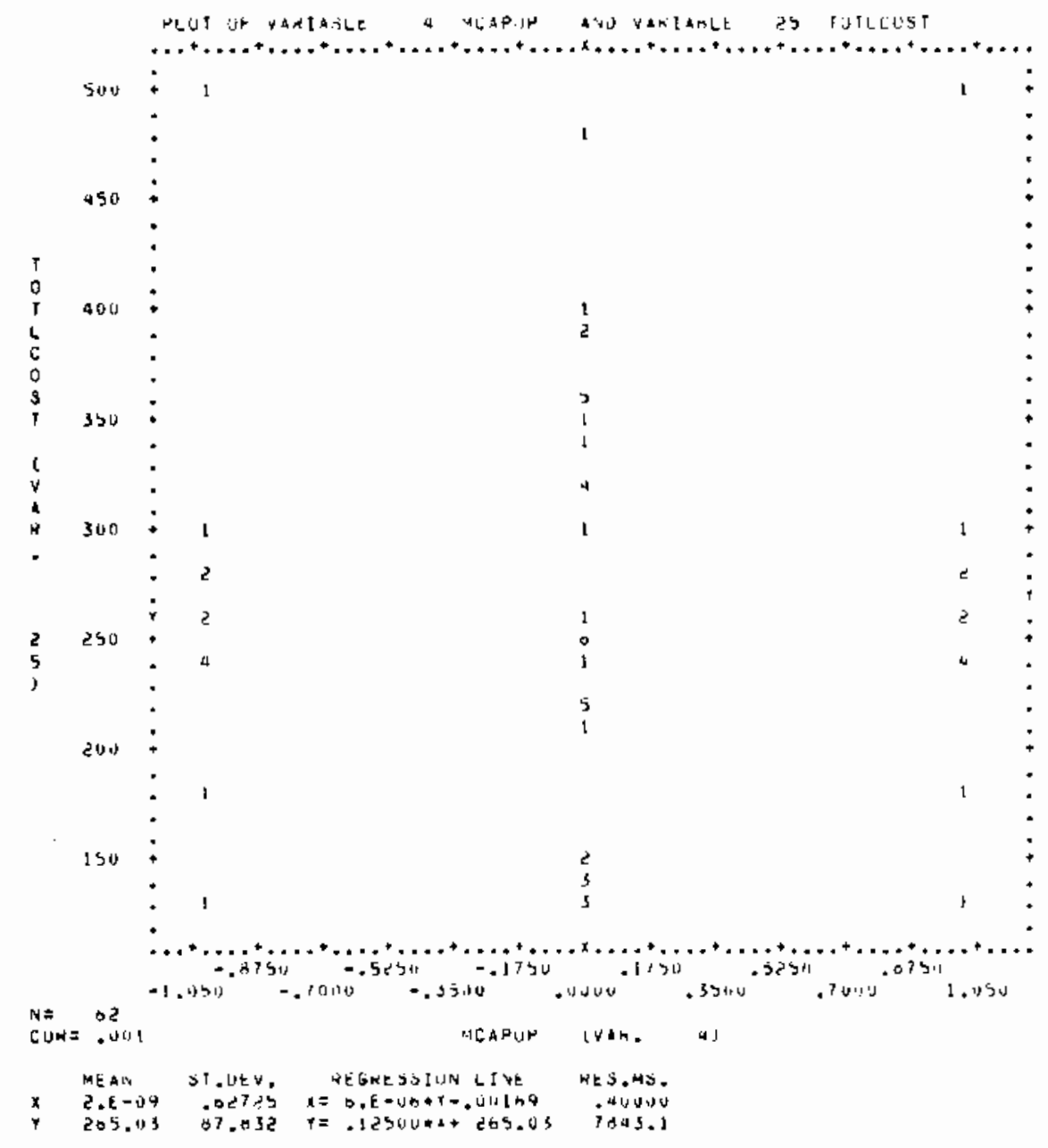

FIGURE C.13 Total Cost Versus Operator's Measurement Capability 


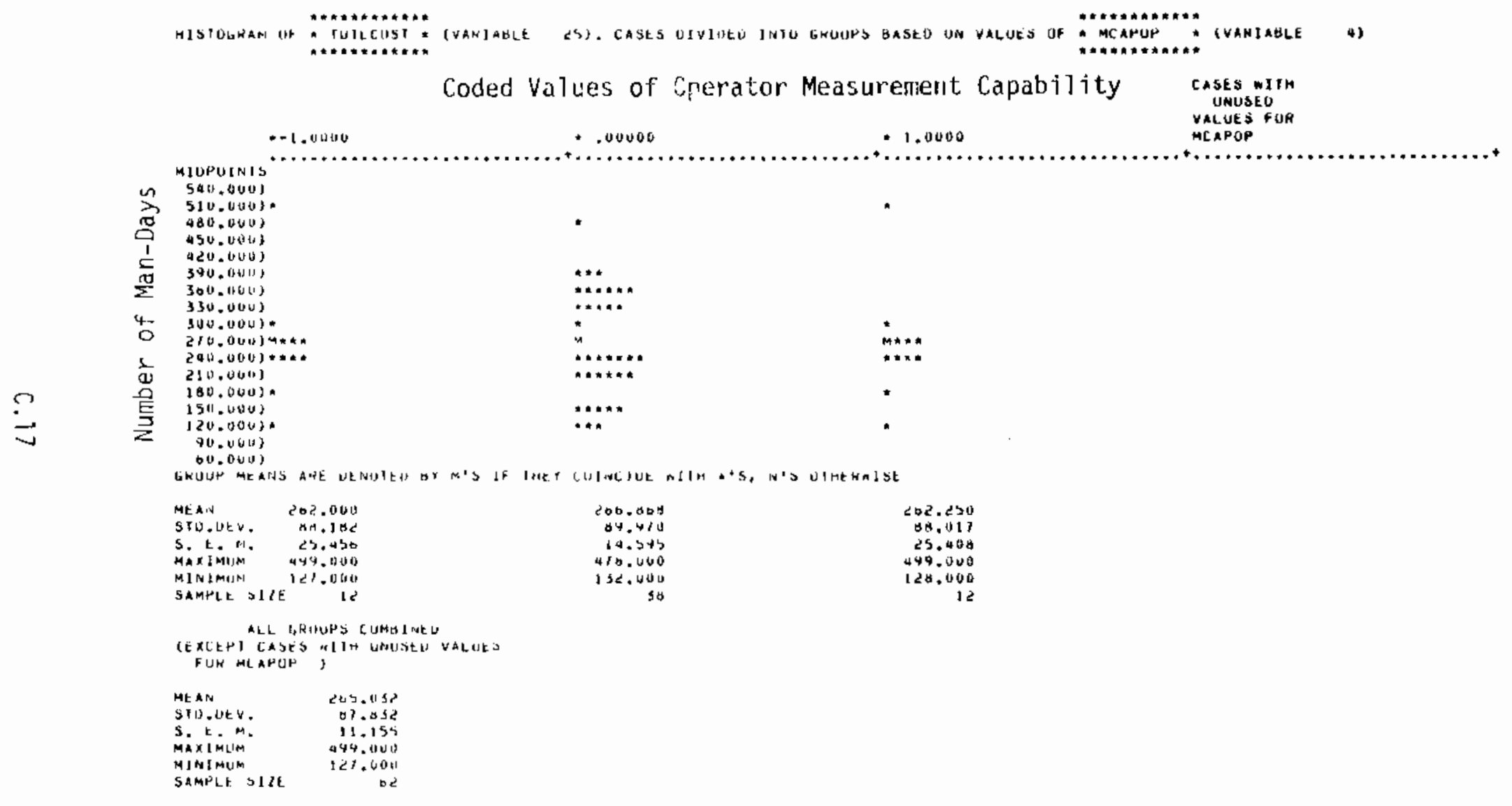

FIGURE C.14 Histograms of Total Cost Versus Operator's Measurement Capability 


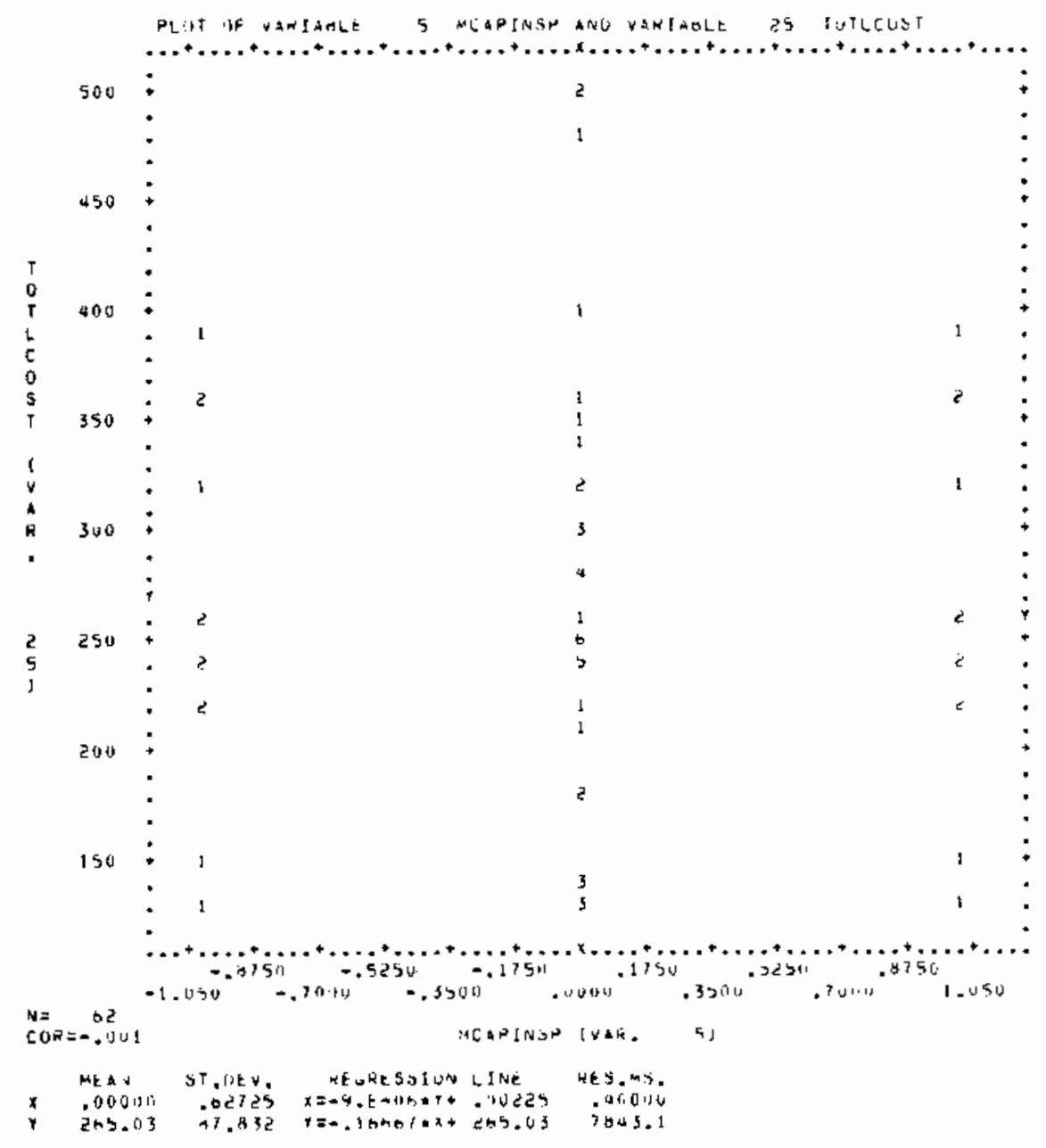

FIGURE C.15 Total Cost Versus Inspector's Measurement Capability 


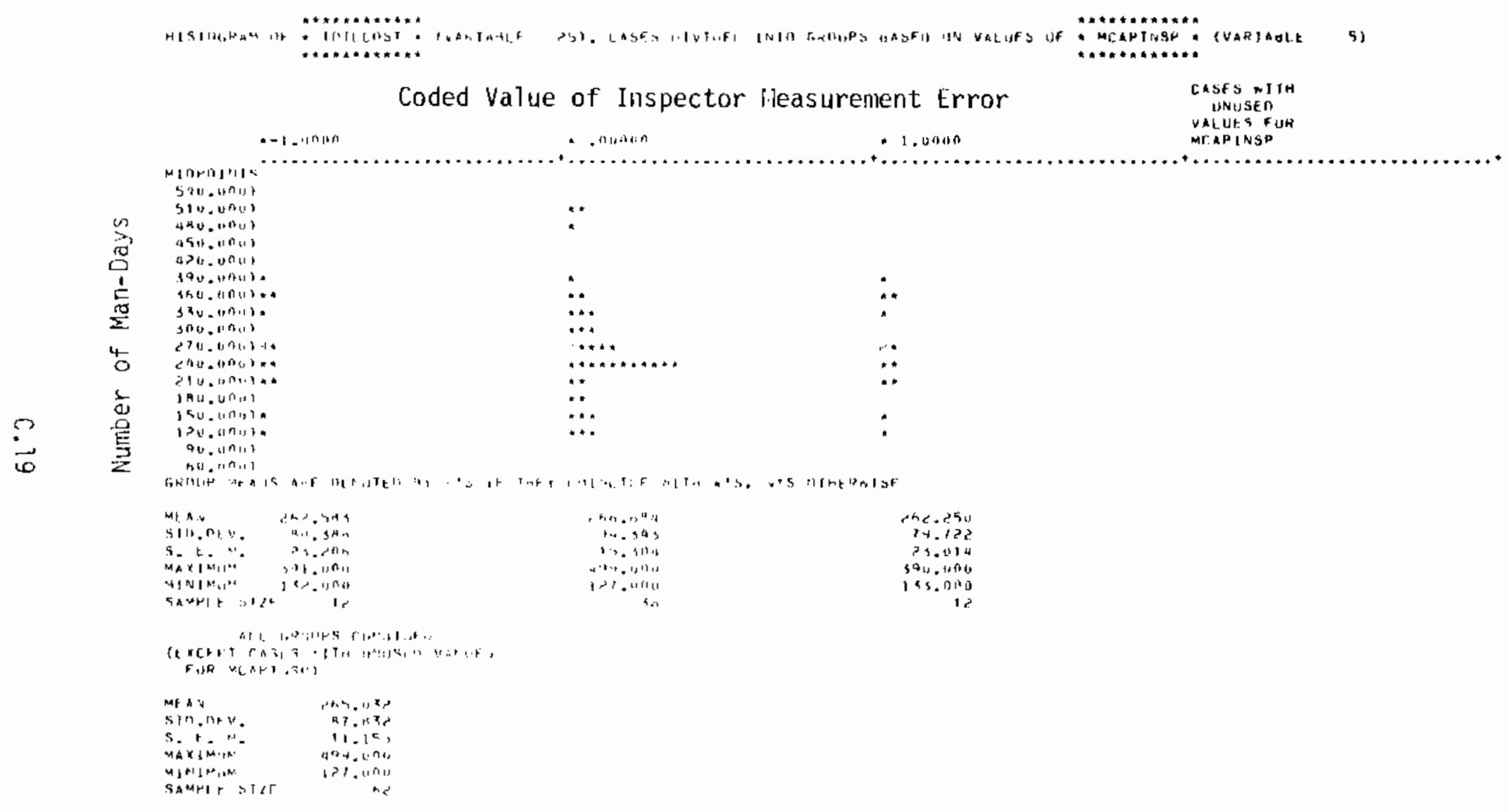

FIGURE C.16 Histograms of Total Cost Versus Inspector's Measurement Capability 


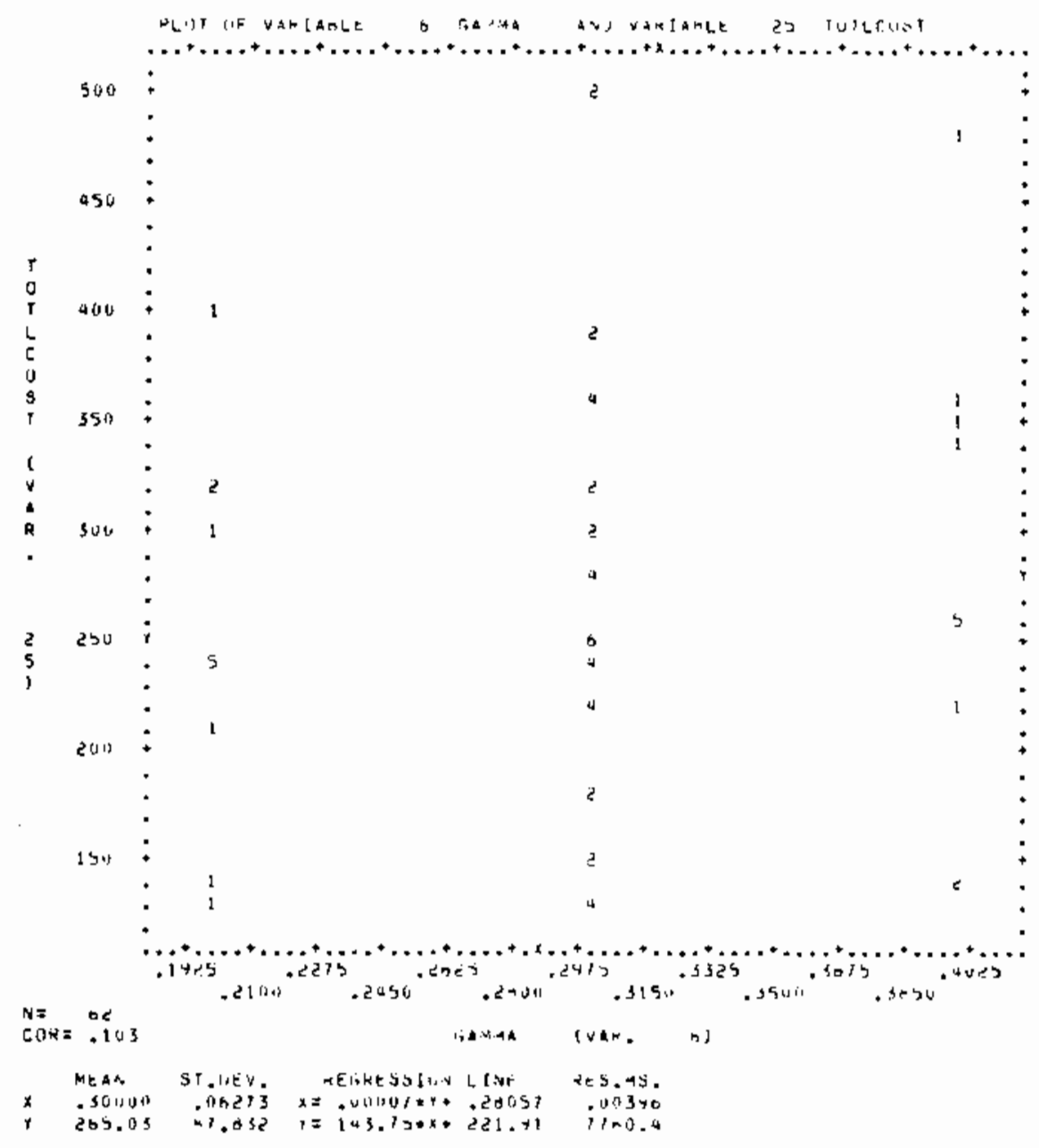

FIGURE C.17 Total Cost Versus Gamma, the Crossover Point 


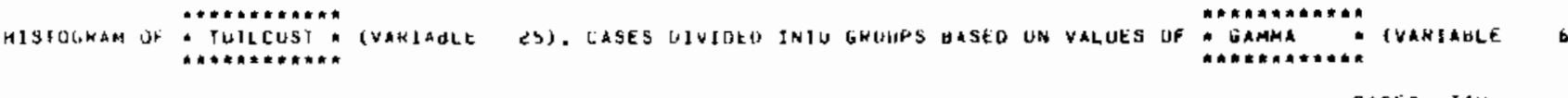

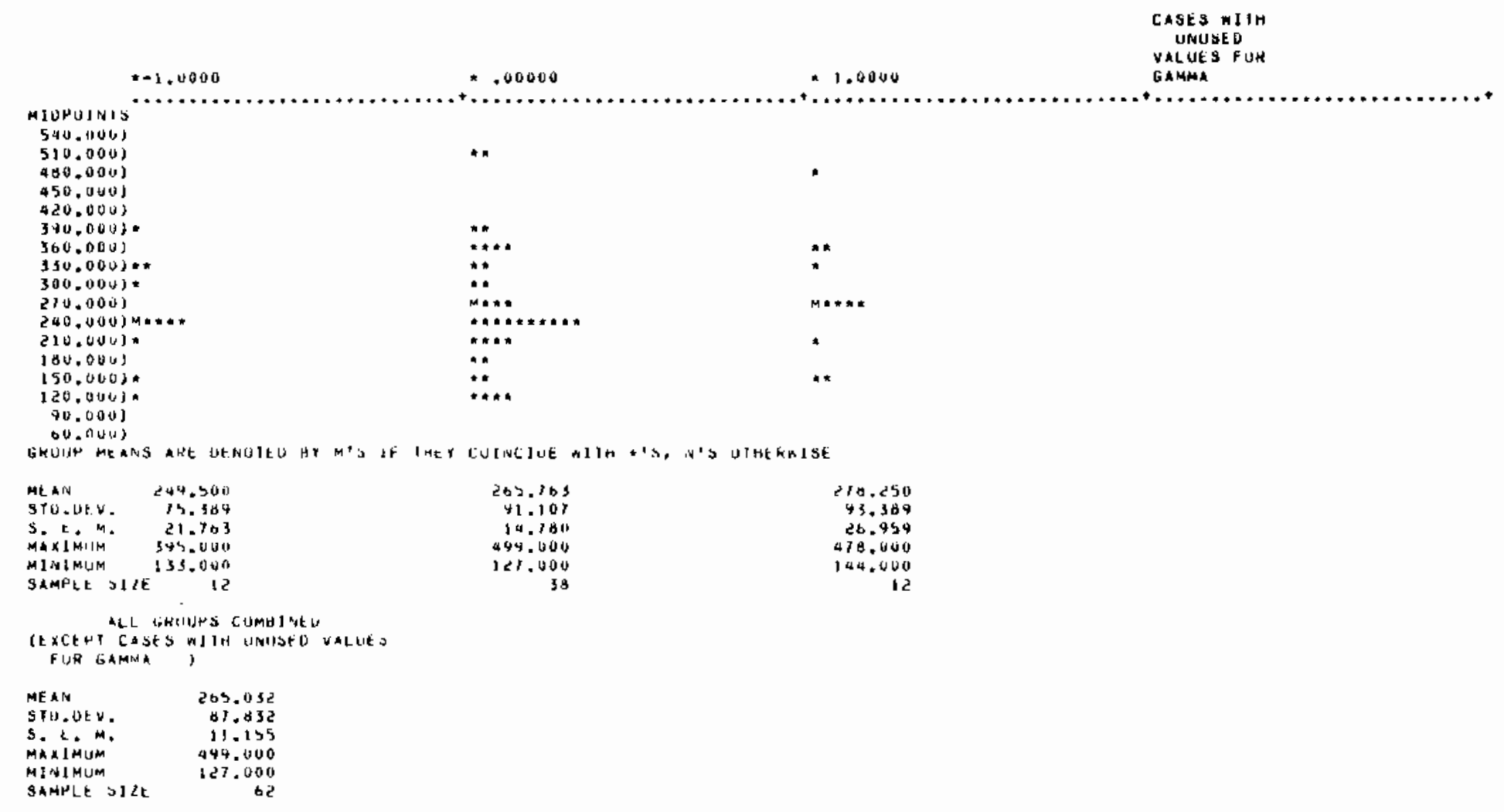

FIGURE C.18 Histograms of Total Cost Versus Gamma, the Crossover Point 
ISPO-139

PNL-3711

UC- -15

DISTRIBUTION

No. of

Copies

OFFSITE

No. of

Copies

\author{
A.A. Churm \\ DOE Chicago Patent Group \\ 9800 South Cass Avenue \\ Argonne, IL 60439 \\ G. Weisz/W.C. Bartels \\ Safeguards \& Security \\ Mai] Code A21016 GTN \\ Department of Energy \\ Washington, DC 20545 \\ S.C.T. McDowel 1 \\ Safeguards \& Security \\ Mail Code A21016 GTN \\ Department of Energy \\ Washington, DC 20545 \\ 27 DOE Technical Information \\ Center \\ 15 Leon Green \\ International Safeguards \\ Project office \\ Building 197 \\ Brookhaven National Laboratory \\ Upton, NY 11973 \\ C.D. Bingham \\ DOE New Brunswick Laboratory \\ Building 350 \\ 9800 South Cass Avenue \\ Argonne, IL 60439 \\ R.L. Heath \\ ISPO Laboratory Coordinator \\ EG\&G Idaho, Inc. \\ Idaho National Engineering \\ Laboratory \\ P.0. Box 1625 \\ Idaho Falis, 1083401
}

D.C. Camp

ISPO Laboratory Coordinator

Lawrence Livermore Laboratory

P.0. Box 808

Livermore, CA 94550

Jack Cusack

ISP0 Laboratory Coordinator

Technical Support Organization

Building 197

Brookhaven National Laboratory

Upton, NY 17973

M.A. Kanter

ISPO Laboratory Coordinator

Argonne National Laboratory

9700 South Cass Avenue

Argonne, IL 60439

H. Menlove

ISPO Laboratory Coordinator

Los Alamos Scientific Laboratory

P.0. Box 1663

Los Alamos, NM 87545

R.A. Tannert

ISPO Laboratory Coordinator

Mail Stop 69

Union Carbide Corporation

P.0. Box P

Oak Ridge, TN 37830

I.G. Waddoups

ISPO Laboratory Coordinator

Sandia Laboratory

P.0. Box 5800

Albuquerque, NM 87715

D. $]$ 
No. of

Copies

5 S. Ermakov

International Atomic Energy Agency

Vienna International Centre

P.0. Box 100

A-1400 Vienna, Austria

T. Shea

International Atomic Energy Agency

Vienna International Centre

P. 0 . Box 100

A-1400 Vienna, Austria

G.C. Hough

International Atomic Energy Agency

Vienna International Centre

P. 0 . Box 100

A-1400 Vienna, Austria

W. Bahm

International Atonic Energy Agency

Vienna International Centre

P. 0. Box 100

A-1400 Vienna, Austria

ON-SITE

2 DOE Richland Operations Office

A.C. Watker

H.E. Ransom

30 Pacific Northwest Laboratory

C.A. Bennett

R.J. Brouns

R.G. Clark

R.F. Eggers

N.L. Harms

M.F. Mullen

P.T. Reardon (15)

B.W. Smith

R.J. Sorenson

Publishing Coordination (2)

Technical Information (5) 